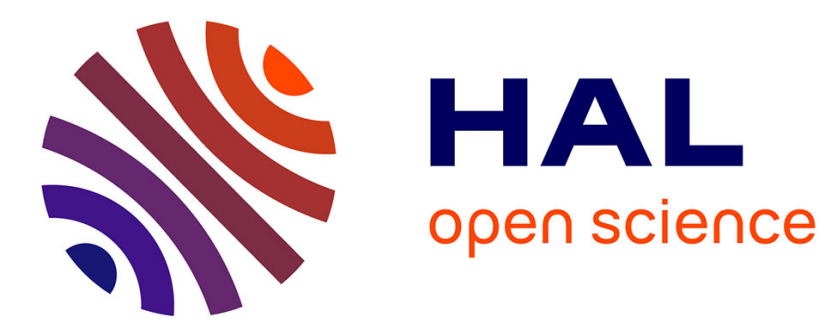

\title{
Diagnostic des systèmes non linéaires - Contribution aux méthodes de découplage
}

\author{
Cédric Join
}

\section{To cite this version:}

Cédric Join. Diagnostic des systèmes non linéaires - Contribution aux méthodes de découplage. Automatique / Robotique. Université Henri Poincaré - Nancy I, 2002. Français. NNT ： . tel-00003393

\section{HAL Id: tel-00003393 \\ https://theses.hal.science/tel-00003393}

Submitted on 18 Sep 2003

HAL is a multi-disciplinary open access archive for the deposit and dissemination of scientific research documents, whether they are published or not. The documents may come from teaching and research institutions in France or abroad, or from public or private research centers.
L'archive ouverte pluridisciplinaire HAL, est destinée au dépôt et à la diffusion de documents scientifiques de niveau recherche, publiés ou non, émanant des établissements d'enseignement et de recherche français ou étrangers, des laboratoires publics ou privés. 


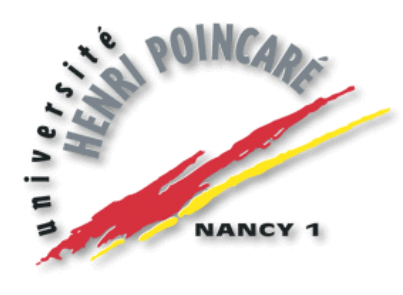

UFR Sciences et Techniques Mathématiques Informatique Automatique Ecole Doctorale IAEM - Lorraine

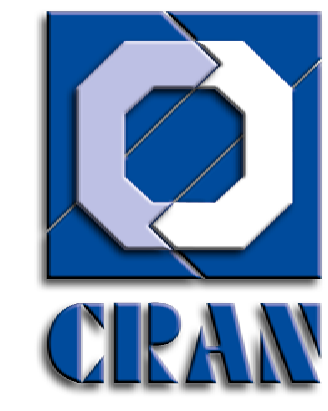

\title{
THĖSE
}

présentée pour l'obtention du

\author{
Doctorat de l'Université Henri Poincaré, Nancy 1 \\ (Spécialité Automatique) \\ par
}

Cédric Join

\section{Diagnostic des systèmes non linéaires Contribution aux méthodes de découplage}

soutenue publiquement le 29 novembre 2002

Composition du jury

Rapporteurs :

M. FLIESS

Directeur de Recherche CNRS à l'Ecole Normale Supérieure, Cachan

A. ZOLGHADRI

Maître de Conférences Habilité à l'Université Bordeaux I, Bordeaux

Examinateurs :

P. MOUYON

Ingénieur de Recherche à l'Office National d'Etudes

et de Recherches Aérospatiales, Toulouse

J. RAGOT

Professeur à l'Institut National Polytechnique de Lorraine, Nancy

J-C. PONSART

Maître de Conférences à l'Université Henri Poincaré, Nancy 1

D. SAUTER

Professeur à l'Université Henri Poincaré, Nancy 1 

A Florence, 



\section{Remerciements}

Le travail présenté dans ce mémoire de thèse a été effectué au Centre de Recherche en Automatique de Nancy (CRAN - CNRS UMR 7039 - Université Henri Poincaré, Nancy). Je remercie F. LEPAGE de m'avoir accueilli au sein de son laboratoire.

Je tiens à remercier les membres du jury qui m'ont fait l'honneur de participer à l'examen de ce travail :

Monsieur M. FLIESS, Directeur de Recherche CNRS au Centre de Mathématiques et Leurs Applications, qui m'a fait l'honneur d'examiner et de rapporter sur ce travail. Je remercie aussi M. FLIESS pour les échanges scientifiques toujours intéressants qui j'espère ne font que débuter.

Monsieur A. ZOLGHADRI, Maître de Conférences HdR au Laboratoire d'Automatique et de Productique, pour le temps consacré à l'examen minutieux de ce travail et ses critiques constructives.

Monsieur P. MOUYON, Ingénieur de Recherche à l'Office National d'Etudes et de Recherches Aérospatiales, de s'être intéressé à ce travail et d'avoir accepté de le juger.

Monsieur J. RAGOT, Professeur à l'Institut National Polytechnique de Lorraine pour l'intérêt qu'il porte à ce travail.

Monsieur D. SAUTER, Professeur à l'Université Henri Poincaré, de m'avoir accueilli au sein de son équipe de recherche et pour sa sympathie.

Monsieur J-C. PONSART, Maître de Conférences à l'Université Henri Poincaré, pour ses conseils de tous les jours et sa confiance. Je tiens particulièrement à le remercier pour sa disponibilité et l'indépendance vitale qu'il a su m'accorder dans mon travail.

Je remercie F. HAMELIN, Maître de Conférences HdR, pour sa lecture attentive du manuscrit. Mais surtout, je tiens à le remercier pour les débats passionnés toujours intéressants et constructifs entre linéariste incertain et non linéariste que je suis. J'espère que nous aurons la possibilité de travailler ensemble dans les années à venir. 
J'exprime ma reconnaissance à A. RICHARD, Professeur à l'Université Henri Poincaré, pour son attention durant les moments difficiles.

Il est maintenant venu de remercier l'ensemble des membres du laboratoire qui contribuent à l'ambiance plus qu'agréable qui y règne.

Je débute par la salle de travail "A" avec :

- Stéphane sans qui je serais bien souvent seul le matin à la "fraîche";

- Hicham pour sa bonne humeur, ses fous rires communicatifs et ses fameuses blagues marocaines ;

- Saïd qui débute sa thèse et que j'apprécie déjà ;

- et bien sûr Fred collègue mais avant tout ami qui défend autant que moi la nécessité de préserver ces salles communes de travail gages de réussites dans la bonne humeur.

Bien sûr je n'oublie pas mes collègues de promotion, Eric et Hadi, et ces longues journées de travail acharné en commun.

Je remercie également les membres du laboratoire qui sont de véritables amis Hassan et son amie Zoubida, Marion et son mari Dom, J-C. et sa femme Aurore, Didier et sa femme Isa, Sabine avec qui il est toujours bon de partager un repas.

Je désire prononcer un mot dédié à chacun donc je "CRIIIEEE" un grand merci à : Manuel, Roudoudou, Olivier, Philippe, Thierry, David, Hugues, Ludo, Jean-Marie, Patrick, Mag, Marc, Mikelkel, Vincent, Christian, Samir, David et Benoit.

Pour finir, mes remerciements les plus profonds vont aux membres de ma famille. Je remercie du fond du coeur ma femme Florence sans qui je ne serais jamais devenu l'étudiant que je fus, qui a su m'assagir et me soutenir tous les jours. Je la remercie particulièrement pour sa patience durant ces 3 longues années.

Je remercie également Papa et Maman qui ont toujours cru en moi, ma soeur Esther et mon frère Goëry et leurs amis respectifs Samuel et Virginie pour leur soutien et les soirées tant attendues entre cousins. Je pense à mes grands-parents d'Epinal et de Valence qui ont toujours su me témoigner leur fierté.

J'exprime aussi mes remerciements à ma belle-famille pour les week-end d'évasion passés au grand air des Vosges. 


\section{Table des matières}

$\begin{array}{ll}\text { Introduction générale } & 11\end{array}$

1 Diagnostic de systèmes non linéaires $\quad 15$

1.1 Introduction au diagnostic . . . . . . . . . . . . . . . . 15

1.2 Du système à la génération de résidus . . . . . . . . . . . . . . . . . . . . . . . . . . . . . . . . . .

1.2.1 Le système et ses entrées . . . . . . . . . . . . . . . . . . . . 17

1.2.1.1 Les défauts . . . . . . . . . . . . . 17

1.2.1.2 Les perturbations . . . . . . . . . . . . . 18

1.2.1.3 Considérations propres au diagnostic . . . . . . . . . 19

1.2.2 Le découplage . . . . . . . . . . . . . . . . . 20

1.2.2.1 Etude du cas linéaire . . . . . . . . . . . 20

1.2.2.2 Problématique liée aux modèles non linéaires . . . . . . . 21

1.2.2.3 Méthode analytique . . . . . . . . . . . . 22

1.2.2.4 Méthode géométrique ............... 27

1.2.2.5 Comparaison des méthodes ............. 30

1.2 .3 Génération de résidus . . . . . . . . . . . . . . . . . 31

1.3 Structuration des résidus . . . . . . . . . . . . . . . . 32

1.3.1 Le problème fondamental de la génération de résidus . . . . . . . . . 32

1.3.2 Structures particulières de banc d'observateurs . . . . . . . . . . . . . 33

1.3.2.1 Structure d'observateurs dédiés . . . . . . . . . . . . . . . . . . . . . . 34

1.3.2.2 Structure d'observateur simplifié . . . . . . . . . . 35

1.3.2.3 Structure d'observateurs généralisés . . . . . . . . . . . 36

1.4 Conclusion et hypothèses de travail . . . . . . . . . . . . . . . . 37

1.4.1 Conclusion . . . . . . . . . . . . . . . 37

1.4 .2 Hypothèses de travail . . . . . . . . . . . . . . . . . 41

2 Découplage : méthode géométrique 43

2.1 Problématique . . . . . . . . . . . . . . . . . . . 43

2.2 Rappel d'une méthode de découplage proposée par C. De Persis . . . . . . . 45

2.2.1 Définition théorique ...................... 45

2.2.2 Exemple académique ..................... 46 
2.3 Proposition d'une méthode de découplage . . . . . . . . . . . . . . 48

2.3.1 Préliminaires . . . . . . . . . . . . . . . . 49

2.3.2 Cas d'une perturbation unique . . . . . . . . . . . 50

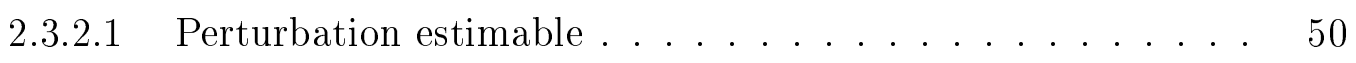

2.3.2.2 Perturbation non estimable ............. 57

2.3.2.3 Conclusion . . . . . . . . . . . . . . . 60

2.3.3 Cas de perturbations multiples ............... 60

2.3.3.1 Perturbations estimables . . . . . . . . . . 61

2.3.3.2 Perturbations non estimables . . . . . . . . . 65

2.3.3.3 Conclusion . . . . . . . . . . . . . 65

2.3.4 Contraintes physiques .................. 66

2.3.5 Exemples académiques . . . . . . . . . . . . . . 67

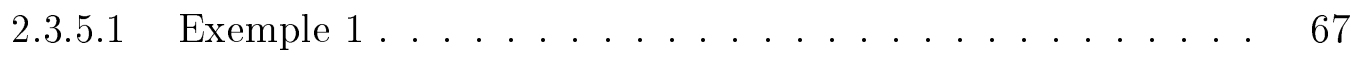

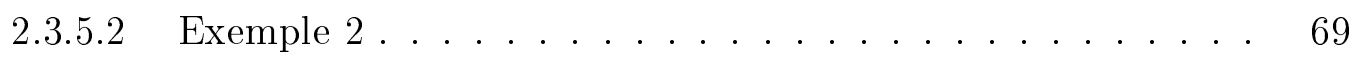

2.4 Analyse structurelle pour le diagnostic . . . . . . . . . . . . . 70

2.4.1 Rappel des objectifs . . . . . . . . . . . . . . . . 71

2.4.2 Proposition de nouvelles conditions de détection et d'isolation de défauts 72

2.4.2.1 Conditions d'existence d'un filtre détecteur de défauts . . . 72

2.4.2.2 Conditions nécessaires à l'isolation des défauts . . . . . . . 73

2.4.2.3 Conditions suffisantes à l'isolation des défauts . . . . . . . . 77

2.4.2.4 Méthodologie ................... 79

2.4.3 Exemples académiques . . . . . . . . . . . . . 80

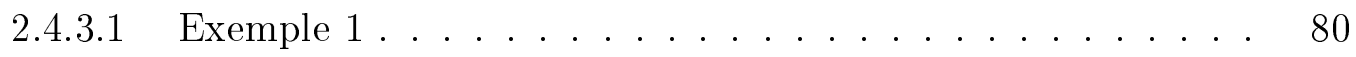

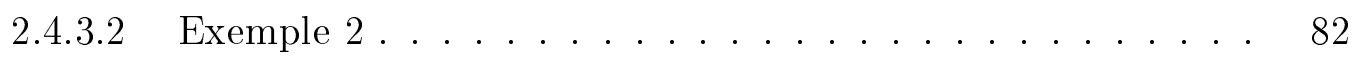

2.5 Conclusion . . . . . . . . . . . . . . . . . . 84

3 Synthèse de filtres non linéaires $\quad 85$

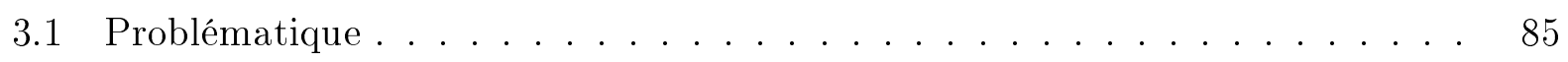

3.2 Calcul de l'injection de sortie pour le découplage . . . . . . . . . . . 87

3.2.1 Calcul de l'injection de sortie dans un cadre particulier . . . . . . . 87

3.2.1.1 Calcul de l'injection de sortie dans le cas multi-filtres . . . . 87

3.2.1.2 Calcul de l'injection de sortie dans le cas mono-filtre . . . . 90

3.2.1.3 Analyse structurelle pour la synthèse d'un filtre d'isolation . 97

3.2.2 Calcul de l'injection de sortie dans un cadre général . . . . . . . . . . 102

3.3 Calcul de l'injection de sortie pour la convergence . . . . . . . . . . . . . . 109

3.3.1 Introduction à la théorie de la contraction . . . . . . . . . . . . . 109

3.3.1.1 "Parallel combination" . . . . . . . . . . . . . . 111

3.3.1.2 "Feedback combination" . . . . . . . . . . . . . 112

3.3.1.3 "Hierarchical combination" . . . . . . . . . . . . . . 112 
3.3.2 Etude de la convergence des filtres d'isolation . . . . . . . . . . . 112

3.3.3 Application de la théorie de la contraction au découplage . . . . . . . 114

3.4 Conclusion . . . . . . . . . . . . . . . . . . 118

4 Application au système des trois cuves $\quad 121$

4.1 Description du système . . . . . . . . . . . . . . . . . . . . . 121

4.2 Modèle mathématique du système . . . . . . . . . . . . . . . . . . 122

4.3 Diagnostic du système . . . . . . . . . . . . . . . . . . . . . 123

4.3.1 Défauts actionneur ...................... 123

4.3.1.1 Isolation à l'aide de plusieurs filtres . . . . . . . . . . . . 124

4.3.1.2 Isolation à l'aide d'un filtre unique . . . . . . . . . . . 127

4.3 .2 Défauts capteur . . . . . . . . . . . . . . . 129

4.4 Analyse de la convergence . . . . . . . . . . . . . . . . 130

4.4.1 Etude de la convergence du filtre isolant un défaut actionneur . . . . 131

4.4.2 Etude de la convergence du filtre isolant tous les défauts actionneur . 134

4.4.3 Etude de la convergence du filtre isolant un défaut capteur . . . . . . 134

4.4.4 Etude de la convergence du filtre isolant tous les défauts capteur . . . 137

4.5 Simulations . . . . . . . . . . . . . . . . . . 138

4.5 .1 Défauts actionneur ....................... 139

4.5 .2 Défauts capteur . . . . . . . . . . . . . . 141

4.6 Conclusion . . . . . . . . . . . . . . . . . . . . 144

5 Ouverture : vers un formalisme algébrique du diagnostic 145

5.1 Introduction . . . . . . . . . . . . . . . . . . . . 145

5.2 Représentation des systèmes linéaires à l'aide de la théorie des modules . . . 146

5.2.1 Définition d'un module approprié aux systèmes linéaires . . . . . . 146

5.2.2 Définition d'un système linéaire à l'aide de la théorie des modules . . 146

5.3 Caractérisation d'un système linéaire . . . . . . . . . . . . . . . 148

5.4 Diagnostic des systèmes linéaires : une approche algébrique . . . . . . . . 150

5.4 Détectabilité des défauts . . . . . . . . . . . . . 150

5.4.1.1 Etude du cas mono-défaut . . . . . . . . . . . . 150

5.4.1.2 Etude du cas multi-défauts . . . . . . . . . . . 151

5.4 .2 Isolabilité des défauts . . . . . . . . . . . . . . . . 152

5.4.2.1 Etude du cas général . . . . . . . . . . . . . . . 152

5.4.2.2 Etude du cas particulier 2_sorties-2_défauts . . . . . . . . . . 154

5.4 .3 Système totalement découplable . . . . . . . . . . . . . 157

5.5 Exemples illustratifs et synthèse de filtres . . . . . . . . . . . . . . . . . 157

5.5 .1 Exemple 1........................ 157

5.5 .2 Exemple $2 \ldots \ldots \ldots \ldots \ldots \ldots \ldots$

5.5 .3 Exemple $3 \ldots \ldots \ldots \ldots \ldots \ldots$ 
5.6 Conclusion . . . . . . . . . . . . . . . . . 159

$\begin{array}{ll}\text { Conclusion générale et perspectives } & 161\end{array}$

$\begin{array}{ll}\text { A Algèbre } & 163\end{array}$

B Géométrie $\quad 165$

$\begin{array}{llr}C & \text { Algèbre commutative } & 167\end{array}$

C.1 Relation d'équivalence . . . . . . . . . . . . . . 167

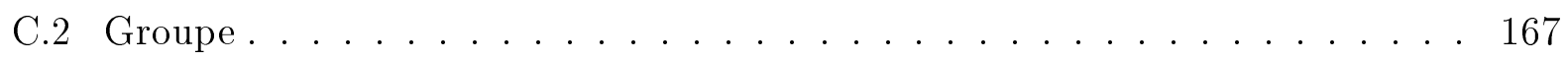

C.3 Anneau . . . . . . . . . . . . . . . . . . 168

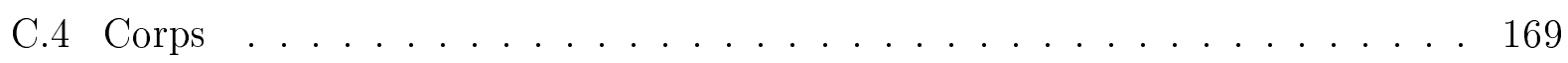

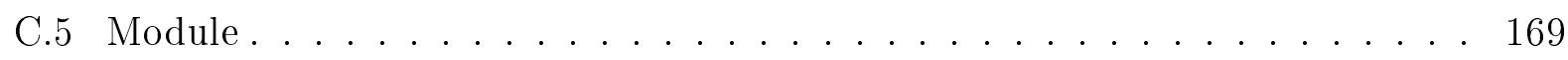

C.6 Morphisme. . . . . . . . . . . . . . . . . 170

C.7 Diagramme commutatif . . . . . . . . . . . . . . . 171

C.8 Foncteur . . . . . . . . . . . . . . . . . . . 171 


\section{Introduction générale}

En raison d'une modernisation incessante des outils de production, les systèmes industriels deviennent de plus en plus complexes et sophistiqués. En parallèle, la fiabilité, disponibilité, sûreté de fonctionnement sans oublier la protection de l'environnement sont devenues de véritables enjeux pour les entreprises actuelles. Le diagnostic des systèmes est apparu dans le but d'améliorer les points précédents. Discipline de l'automatique à part entière, ce module de surveillance qu'est le diagnostic fait l'objet d'un engouement prononcé depuis le début des années 70. En effet, la recherche dans ce domaine n'a fait que prendre de l'importance dans le monde entier, aussi bien de manière théorique que pratique.

Les méthodes de diagnostic que nous considérons reposent sur la connaissance d'un modèle du système. Issu d'une phase d'identification, le modèle du système a pour objectif principal de représenter au mieux le comportement réel du système en dépit de tout élément perturbateur extérieur ou de mauvaises connaissances a priori (modèle de type boite noire ou grise). Les modèles considérés sont issus d'équations différentielles et/ou basés sur la connaissance des équations physiques.

La majeure partie des systèmes réels obéissent à des équations non linéaires. Les modèles non linéaires des systèmes sont alors communément linéarisés autour d'un point de fonctionnement afin de posséder des propriétés linéaires. Ainsi les nombreuses méthodes développées depuis une trentaine d'année peuvent être appliquées. Cependant cette étape de linéarisation est la cause principale d'erreurs de modélisation. Par conséquent l'étude des modèles linéaires de représentation incertains est inévitable.

Depuis les années 80 les approches non linéaires sont développées pour la commande de ces systèmes. Issues de ces dernières, le découplage d'une partie de l'état de l'effet des perturbations joue un rôle d'importance pour le diagnostic des systèmes non linéaires. De plus puisque la première cause d'incertitude est évincée, il parait moins utopique de considérer des modèles parfaits. Dans cette thèse nous supposons la modélisation du système parfaitement réalisée.

Notons que le point commun à toutes les approches linéaires ou non linéaires pour le diagnostic est la synthèse d'un signal nommé résidu. Ce signal, s'appuyant sur la redondance 
analytique qu'offre le modèle, est généralement une fonction des sorties mesurées du système et de ses entrées. L'object principal d'un résidu est d'assurer la distinction entre le fonctionnement normal du système et un fonctionnement anormal dû à l'apparition d'un défaut d'une part et de différentier l'effet des différents défauts entre eux d'autre part. Parmi un nombre toujours croissant d'outils assurant cet objectif, nous distinguons les relations de parités, les observateurs et les filtres. Ces deux derniers possèdent l'avantage certain d'estimer les états et les sorties du système.

Le travaille présenté dans ce mémoire contribue à enrichir tant les méthodes visant l'analyse des systèmes non linéaires pour aboutir à des conditions d'isolation des défauts que les méthodes de synthèse de filtre pour la génération de résidus.

Ce mémoire comporte 5 chapitres.

Le premier chapitre présente précisément les principes fondamentaux sur lesquels repose le diagnostic. Puisque toute méthode visant l'isolation de défauts nécessite une phase de découplage, nous détaillons et comparons les différentes méthodes proposées dans la littérature. Ces méthodes permettent la structuration des résidus. Pour clore ce chapitre, plusieurs structures particulières de résidus sont détaillées.

Se fondant sur une nouvelle écriture des filtres nous proposons, dans le deuxième chapitre, un calcul novateur du plus petit sous-espace d'état sensible à une perturbation. Cette modification a pour objectif d'augmenter significativement la dimension du sous-espace d'état insensible à une perturbation et donc la partie de l'état qui sera correctement estimée malgré l'apparition de cette perturbation. Suite à l'obtention de l'ensemble de ces sous-espaces d'état insensibles aux défauts, une analyse approfondie de leurs caractéristiques géométriques nous permet d'établir de nouvelles conditions pour l'isolation des défauts.

En lien avec les résultats du chapitre précédent, dans le troisième chapitre nous proposons une méthode systématique de synthèse des filtres. Différents cas sont présentés. Les deux principaux sont l'isolation de l'ensemble des défauts à l'aide d'un unique filtre d'une part et d'un banc de filtres d'autre part. Nous complétons la synthèse de ces filtres d'une étude menant à l'assurance de la convergence exponentielle de l'estimation des états vers les états réels du système.

Dans le quatrième chapitre l'ensemble des méthodes proposées sont illustrées avec l'étude du benchmark des trois cuves. Ainsi, des défauts capteur et actionneur sont considérés. Cette application met en relief les résultats de nos propositions tant au niveau du découplage que de la génération de résidu.

Le dernier chapitre a pour objectif la présentation de nouveaux outils qui offrent des perspectives intéressantes pour le diagnostic. En effet, très récemment différentes méthodes de synthèse de lois de commande ayant pour essence la théorie des modules sont apparues (nous citons à titre d'exemple, la platitude et les P.I.D. généralisés). Ainsi, une étude algébrique 
est menée afin d'établir les conditions nécessaires et suffisantes d'isolation des défauts. Ces conditions sont intrinsèques au modèle du système et ne dépendent donc pas de la forme du générateur de résidus choisi. 



\section{Chapitre 1}

\section{Diagnostic de systèmes non linéaires}

Ce chapitre a pour objet la présentation des principaux concepts et outils abordés dans la suite de ce document.

Notre intérêt se portant sur le diagnostic des systèmes décrits par des modèles non linéaires, nous débutons cette présentation par une description des particularités associées à différents types de modèles. Ensuite, nous détaillons la démarche menant au diagnostic de systèmes. En effet, le diagnostic de systèmes (la détection et l'isolation de défauts) nécessite une succession d'étapes imbriquées. Une étude bibliographique détaillée permet une présentation de chacune d'entre elles.

\subsection{Introduction au diagnostic}

Les travaux décrits dans ce manuscrit sont basés sur les possibilités de redondance analytique offertes par un système dynamique. La redondance analytique représente la possibilité de traduire à l'aide d'équations mathématiques le comportement du (ou d'une partie du) système. Ces équations peuvent être statiques (1.1) ou dynamiques (1.2). A cette dernière équation, nous associons un modèle plus fin du système, traduisant le régime transitoire à l'aide d'équations différentielles. Nous considérerons, par la suite, ce dernier type de modèle.

$$
\begin{gathered}
y(t)=K x(t) \\
\dot{x}(t)=f(x(t), u(t))
\end{gathered}
$$

Au diagnostic de systèmes, nous associons les deux tâches suivantes : la détection et l'isolation des défauts [36].

Définition 1.1 Détection de défauts : détermination de la présence de défauts sur un système et de l'instant d'apparition.

Définition 1.2 Isolation de défaut: détermination du type, de l'emplacement et de l'instant d'apparition d'un défaut. Suit la détection de défauts. 
Une troisième tâche peut y être ajoutée : l'estimation de l'amplitude du défaut. La réalisation de ces trois tâches nécessite de différencier le fonctionnement normal du système d'un fonctionnement anormal ou dégradé.

A cet effet, nous utilisons des modèles qui représentent avec plus ou moins de précision le comportement réel du système. Ces modèles peuvent être classifiés [25], par ordre de généralité :

- les modèles non linéaires généraux,

$$
\Sigma_{N L G}:\left\{\begin{array}{l}
\dot{x}(t)=f(x(t), u(t)) \\
y_{x}(t)=h(x(t))
\end{array}\right.
$$

- les modèles non linéaires bilinéaires,

$$
\Sigma_{N B}:\left\{\begin{array}{l}
\dot{x}(t)=f_{0}(x(t))+\sum_{i=1}^{m} f_{i}(x(t)) u_{i}(t) \\
y_{x}(t)=h(x(t))
\end{array}\right.
$$

- les modèles non linéaires affines en la commande,

$$
\Sigma_{N L}:\left\{\begin{array}{l}
\dot{x}(t)=f_{0}(x(t))+\sum_{i=1}^{m} f_{i}(x(t)) u_{i}(t) \\
y_{x}(t)=h(x(t))
\end{array}\right.
$$

- les modèles linéaires,

$$
\Sigma_{L}:\left\{\begin{array}{l}
\dot{x}(t)=A x(t)+B u(t) \\
y_{x}=C x(t)
\end{array}\right.
$$

où $x(t) \in \mathbb{R}^{n}, u(t) \in \mathbb{R}^{m}$, et $y_{x}(t) \in \mathbb{R}^{p}$ représentent respectivement les vecteurs d'état, entrée et sortie observées du système.

Pour être exploitable, au vu des objectifs de diagnostic que nous nous sommes fixés (définitions 1.1 et 1.2), le modèle doit traduire au mieux le comportement du système. Cependant, la précision d'un modèle est généralement obtenue au détriment de sa simplicité. Le choix entre :

- les modèles linéaires pour lesquels de nombreuses méthodes de traitement sont connues mais possédant une plage de fonctionnement limitée,

- les modèles non linéaires d'utilisation complexe mais possédant un domaine de validité étendu,

est difficile à effectuer. Lors des chapitres suivants, nous contribuons au développement des méthodes associées aux modèles non linéaires affines en la commande. Cependant, il convient de signaler une solution relativement récente, qui consiste à multiplier le nombre de modèles linéaires couvrant au mieux les trajectoires du système. Cette stratégie connue sous le nom 
de multi-modèles fait l'objet de nombreuses publications visant la résolution de nombreux problèmes qui lui sont inhérents ([55], [32], [54], [1]) : commutation entre modèles, distinction entre changement de consigne et apparition d'un défaut, ...

Dans la section suivante, nous précisons l'ensemble des éléments nécessaires pour mener à bien le diagnostic de système. Nous la débutons en précisant le modèle du système considéré en y intégrant les défauts. Ensuite, puisque l'objectif d'isoler les défauts, à partir de résidus, ne peut être atteint que lorsque ces derniers possèdent des propriétés différentes, nous étudions deux méthodes de découplage intéressantes proposées dans la littérature.

\subsection{Du système à la génération de résidus}

En ce qui nous concerne, nous intégrons les défauts et perturbations dans la modèlisation d'un système non linéaire. En effet, modéliser les effets d'autres entrées (défauts et perturbations) que la commande est primordial pour le diagnostic de système. Cette modèlisation se justifie par la nécessité de différencier les défauts et surtout d'assurer l'insensibilité d'une partie de l'état du modèle (dans la mesure du possible) aux perturbations.

\subsubsection{Le système et ses entrées}

Dans cette section, nous précisons le terme "d'entrées inconnues" (entrées exogènes) qui représentent les grandeurs physiques ayant un effet sur le système sans qu'elles soient mesurées. Nous les détaillons en nous référant à la littérature. Pour débuter, nous définissons différents types de défauts et de perturbations. Puis, nous éclaircissons certaines considérations usuelles dans le cadre du diagnostic.

\subsubsection{Les défauts}

Nous distinguons trois classes de défauts qui interviennent à différents niveaux sur le système (figure 1.1) :

- défaut actionneur : défaut $w_{a}(t)$ qui agit au niveau de la partie opérative et détériore ainsi le signal d'entrée du système (perte de puissance d'un moteur, fuite dans un vérin, ...).

- défaut système : nous identifions le défaut $w_{s}(t)$ à la modification des caractéristiques du système proprement dit. Une fuite dans une cuve, le vieillissement des matériaux en sont des exemples simples,

- défaut capteur : un tel défaut $w_{c}(t)$ est la cause d'une mauvaise image de l'état physique du système (mauvais étalonnage de capteur ou vieillissement de l'organe récepteur d'un capteur, ...). 


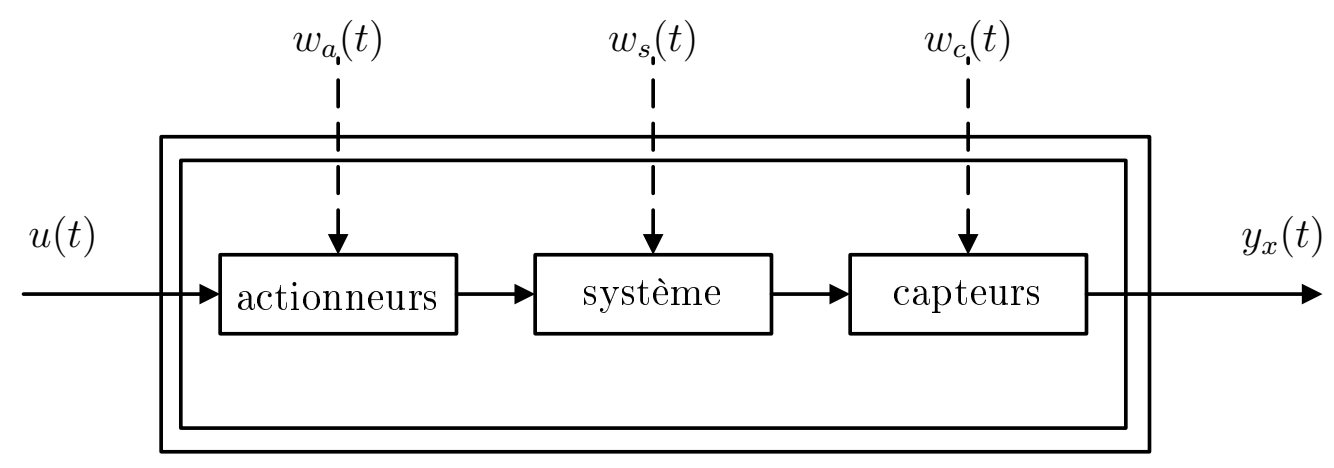

FIG. 1.1: Inventaire des défauts agissant sur un système

De manière à distinguer ces différentes classes de défauts, nous récrivons le modèle non linéaire affine en la commande :

$$
\Sigma_{N L}:\left\{\begin{array}{l}
\dot{x}(t)=f_{0}(x(t))+\sum_{k=1}^{r} f_{s_{k}}(x(t)) w_{s_{k}}(t)+\sum_{i=1}^{m} f_{i}(x(t))\left(u_{i}(t)+w_{a_{i}}(t)\right) \\
y_{x}(t)=h(x(t))+\sum_{j=1}^{p} f_{c_{j}} w_{c_{j}}(t), \text { avec } f_{c_{j}} \text { la } j^{\text {e }} \text { ligne d'une matrice diagonale }
\end{array}\right.
$$

$w_{a}(t), w_{s}(t)$ et $w_{c}(t)$ représentent respectivement les vecteurs constitués des défauts actionneur, système et capteur. Cependant, nous ajoutons que lorsque la structure même d'un actionneur est modifiée suite à l'apparition d'un défaut, ce dernier est nécessairement une composante du vecteur $w_{s}(t)$.

D'autres entrées peuvent également modifier le comportement du système. La section suivante est consacrée à leur étude.

\subsubsection{Les perturbations}

Les utilisations du modèle (1.7) sont diverses (diagnostic, commande, identification pour les principales), cependant l'adjonction d'un modèle des perturbations, notées $d(t)$, est généralement réalisée :

$\Sigma_{N L}:\left\{\begin{array}{l}\dot{x}(t)=f_{0}(x(t))+\sum_{k=1}^{r} f_{s_{k}}(x(t)) w_{s_{k}}(t)+\sum_{i=1}^{m} f_{i}(x(t))\left(u_{i}(t)+w_{a_{i}}(t)\right)+D_{e}(x(t)) d(t) \\ y_{x}(t)=h(x(t))+\sum_{j=1}^{p} f_{c_{j}} w_{c_{j}}(t)+D_{s}(x(t)) d(t)\end{array}\right.$

L'origine de ces perturbations est souvent due à une modélisation imparfaite du système. En effet, une perturbation est une grandeur ayant une action, que l'on ne connait pas, sur le système. Ceci s'explique par le fait que nous avons omis de prendre en compte la perturbation lors de la phase de modélisation. 
Ces perturbations font parfois l'objet de caractéristiques particulières. Ainsi, lorsque ces dernières sont d'ordre statistique, le système est dit bruité. Cet axe de recherche (stochastique) est particulier et a fait l'objet de peu d'études dans le cas des systèmes non linéaires ([17], [5]).

Dans ce document, nous n'effectuons pas de synthèse visant la minimisation de l'effet des perturbations au sens stochastique. Nos travaux seront donc dits déterministes.

\subsubsection{Considérations propres au diagnostic}

Par souci d'uniformité, dans le cas déterministe grâce à une manipulation mathématique conduisant à une augmentation d'état, il est possible d'écrire toute entrée inconnue au niveau de l'équation d'état du système ([52] et [57]). Nous précisons, que si les entrées inconnues (perturbation $d(t)$ et/ou défaut capteur $w_{c}(t)$ ) font preuves de discontinuités, il n'est alors possible d'en réaliser qu'une approximation (sans que cela soit gênant).

Ainsi, dans la suite de nos travaux, aucune distinction n'est faite entre les différentes écritures (1.8) et (1.9). Nous considérons alors, le système suivant comme base de réflexion :

$$
\Sigma_{N L}:\left\{\begin{array}{l}
\dot{x}(t)=f_{0}(x(t))+\sum_{i=1}^{m} f_{i}(x(t)) u_{i}(t)+\sum_{i=1}^{m+p+r=q} P_{i}(x(t)) w_{i}(t)+\sum_{i=1}^{s} D_{x i}(x(t)) d_{i}(t) \\
y_{x}(t)=h(x(t))
\end{array}\right.
$$

avec $w(t)$ l'écriture du vecteur défaut constitué par tous les défauts (actionneur, système et capteur), $d(t)$ le vecteur perturbation et $P(x(t)), D_{x}(x(t))$ les matrices qui les pondérent.

Contrairement aux objectifs d'une loi de commande, où l'effet des défauts et des perturbations est minimisé (aucune distinction dans leur traitement) vis à vis des signaux de sorties à contrôler, le diagnostic a pour finalité la mise en exergue d'un ou plusieurs défauts quelles que soient les perturbations. Ainsi, lors de l'écriture du système (1.9), nous donnons un autre sens à :

- $w(t)$ qui correspond aux défauts que nous voulons détecter (et isoler si c'est possible),

- $d(t)$ qui correspond aux autres défauts et éventuellement perturbations.

Ainsi la problématique du diagnostic est la génération d'un résidu $r(t)$ (signal multidimensionnel) vérifiant :

- $r(t) \neq 0$ si $w_{i}(t) \neq 0 \forall i$, quelles que soient les valeurs prises par les autres entrées du système $u(t)$ et $d(t)$,

- $r(t)=0$ si $w(t)=0$, quelles que soient les valeurs prises par les autres entrées du système $u(t)$ et $d(t)$. 
L'obtention d'un tel signal $r(t)$ fait l'objet de plusieurs étapes dont le découplage et la structuration des résidus, pour ne citer que les principales. L'étape de découplage est à présent détaillée.

\subsubsection{Le découplage}

Nous associons le découplage à l'opération consistant à rendre totalement insensible, sous l'action d'un filtre, une partie de l'état (sous-espace d'état) à une entrée préalablement déterminée $(d(t))$. Suite à cette remarque nous définissons un sous-espace d'état insensible (resp. sensible) :

Définition 1.3 Un sous-espace d'état est dit insensible (resp. sensible) à une entrée s'il existe un (resp. n'existe pas de) filtre assurant une estimation parfaite de l'état associé à ce sous-espace quelle que soit cette entrée.

Avant d'aborder l'étude des modèles non linéaires, nous développons le cas linéaire. Deux méthodes non linéaires distinctes sont ensuite détaillées.

\subsubsection{Etude du cas linéaire}

Le modèle du système considéré, sous les conditions d'absence de bruit, de défauts et d'absence totale d'incertitude de modèle, est de la forme suivante :

$$
\Sigma_{L}:\left\{\begin{array}{l}
\dot{x}(t)=A x(t)+B u(t)+D_{x} d(t) \\
y_{x}(t)=C x(t)
\end{array}\right.
$$

où les matrices $A, B, C$ et $D_{x}$ sont constantes, $D_{x}$ de rang plein colonne et $\operatorname{dim}(d(t))=s$. L'objectif est le découplage d'une partie de l'état de l'effet de $d(t)$. Nous présentons une méthode basée sur l'utilisation de deux décompositions en valeurs singulières (Singular Value Decomposition, S.V.D. en anglais) ([10], [18]).

Suite à une première S.V.D. de $D_{x}$, il est possible d'exprimer l'état du modèle (1.10) de la manière suivante :

$$
\left\{\begin{array}{l}
\dot{\tilde{x}}_{1}(t)=A_{11} \tilde{x}_{1}(t)+A_{12} \tilde{x}_{2}(t)+B_{1} u(t)+D_{x 1} d(t) \\
\dot{\tilde{x}}_{2}(t)=A_{21} \tilde{x}_{1}(t)+A_{22} \tilde{x}_{2}(t)+B_{2} u(t)
\end{array}\right.
$$

où $\tilde{x}(t)=\left(\tilde{x}_{1}^{T}(t) \tilde{x}_{2}^{T}(t)\right)^{T}=H x(t)$ avec $H$ la matrice de passage du changement de coordonnées.

Une deuxième S.V.D. de $C$ permet l'isolation de $\tilde{x}_{1}(t)$ au sein de l'équation de mesure.

$$
\left\{\begin{array}{l}
y_{x 1}(t)=C_{11} \tilde{x}_{1}(t)+C_{12} \tilde{x}_{2}(t) \\
y_{x 2}(t)=C_{2} \tilde{x}_{2}(t)
\end{array}\right.
$$


Ainsi, exprimer $\tilde{x}_{1}(t)$ en fonction de $y_{x}(t)$ et $\tilde{x}_{2}(t)$, est possible. Cette étape permet d'extraire un sous-système insensible à $d(t)$ s'écrivant comme suit :

$$
\Sigma_{\text {Linsensible }}:\left\{\begin{array}{l}
\dot{\tilde{x}}_{2}(t)=\left(A_{22}-A_{21} C_{11}^{-1} C_{12}\right) \tilde{x}_{2}(t)+B_{2} u(t)+A_{21} C_{11}^{-1} y_{x 1}(t) \\
y_{x 2}(t)=C_{2} \tilde{x}_{2}(t)
\end{array}\right.
$$

Or différentes hypothèses sont nécessaires à cette écriture :

- $\operatorname{dim}(d(t))<\operatorname{dim}\left(y_{x}(t)\right)$ : le nombre de défauts est moins important que le nombre de sorties,

- $\operatorname{dim}\left(\operatorname{span}\left\{C D_{x}\right\}\right)=\operatorname{dim}(d(t)):$ chaque défaut a un effet différent sur les sorties.

Notons que la dimension du sous-système représenté par $(1.13)$ est $\operatorname{dim}\left(\tilde{x}_{2}(t)\right)=n-s$.

L'étude du cas linéaire permet la mise en évidence des trois étapes qui constituent le découplage de $d(t)$ :

- un premier changement de coordonnées pour scinder en deux parties l'état du modèle $\left(\tilde{x}_{1}(t)\right.$ et $\left.\tilde{x}_{2}(t)\right)$ qui, respectivement, font et ne font pas intervenir dans l'écriture de l'équation différentielle le signal $d(t)$,

- un deuxième changement de coordonnées qui distingue les sorties dépendant de $\tilde{x}_{1}(t)$ $\left(y_{x 1}(t)\right)$ des autres sorties,

- la dernière étape est l'expression de $\tilde{x}_{1}(t)$ à l'aide de $\tilde{x}_{2}(t)$ et $y_{x 1}(t)$. Cette expression notée $L\left(\tilde{x}_{2}^{T}(t) y_{x}^{T}(t)\right)^{T}$, peut s'interpréter comme un retour (ou injection) de sortie.

Suite à ce dernier point, l'équation d'état du modèle (1.13) s'écrit :

$$
\dot{\tilde{x}}_{2}(t)=A_{22} \tilde{x}_{2}(t)+B_{2} u(t)+A_{21} L\left(\tilde{x}_{2}^{T}(t) y_{x}^{T}(t)\right)^{T}
$$

avec $\tilde{x}_{1}(t)=L\left(\tilde{x}_{2}^{T}(t) y_{x}^{T}(t)\right)^{T}=C_{11}^{-1}\left(y_{x 1}(t)-C_{12} \tilde{x}_{2}(t)\right)$.

Ainsi le gain $L$ représente un retour de sortie (ou injection de sortie) et constitue l'élément principal du découplage.

Nous ajoutons que dans le cadre du découplage des systèmes linéaires, il existe également une autre méthode intéressante détaillée plus en avant du manuscrit (paragraphe 1.2.2.4) se fondant sur l'utilisation de la géométrie différentielle et plus particulièrement les notions de sous-espaces invariants ([65]).

Nous rappelons maintenant le problème propre aux modèles non linéaires.

\subsubsection{Problématique liée aux modèles non linéaires}

Le modèle non linéaire du système considéré est :

$$
\Sigma_{N L}:\left\{\begin{array}{l}
\dot{x}(t)=f(x(t), u(t))+D_{x}(x(t), u(t)) d(t) \\
y_{x}(t)=h(x(t))
\end{array}\right.
$$


avec $\operatorname{dim}(d(t))=s$.

Nous allons suivre le même schéma que celui énoncé dans le paragraphe précédent. Nous cherchons un changement de base particulier permettant une écriture équivalente à (1.11) du système non linéaire (1.15) :

$$
\left\{\begin{array}{l}
\dot{\tilde{x}}_{1}(t)=\tilde{f}_{1}\left(\tilde{x}_{1}(t), \tilde{x}_{2}(t), u(t)\right)+\tilde{D}_{x 1}\left(\tilde{x}_{1}(t), \tilde{x}_{2}(t), u(t)\right) d(t) \\
\dot{\tilde{x}}_{2}(t)=\tilde{f}_{2}\left(\tilde{x}_{1}(t), \tilde{x}_{2}(t), u(t)\right)
\end{array}\right.
$$

avec $\tilde{x}(t)=\Phi_{d}(x(t), u(t))$.

$\Phi_{d}(x(t), u(t))$ est une transformation d'état définie par l'équation suivante :

$$
\frac{\partial \Phi_{d}(x(t), u(t))}{\partial x(t)} D_{x}(x(t), u(t))=0
$$

dont l'égalité est satisfaite $\forall(x(t), u(t))$. La condition nécessaire et suffisante pour résoudre (1.17) est donnée par le théorème de Frobenius (voir annexe A théorème A.1).

Le problème propre aux modèles non linéaires réside dans la détermination de l'injection de sortie visant à exprimer le sous-espace d'état inclus dans celui correspondant à $\tilde{x}_{1}(t)$ et nécessaire à l'expression de $\dot{\tilde{x}}_{2}(t)$. L'écriture de cette fonction non linéaire $\Psi\left(\tilde{x}_{2}(t), \bullet\right)$ (où • représente des sorties, des commandes et/ou leurs dérivées suivant les méthodes de détermination employées) est l'analogue à $L\left(\tilde{x}_{2}^{T}(t) y_{x}^{T}(t)\right)^{T}$ en linéaire, et nécessite une séparation des variables d'état au niveau de l'équation de sortie.

Notons que cette étape, contrairement au cas des modèles linéaires, n'est ni immédiate, ni toujours possible avec des modèles non linéaires. Il s'agit de déterminer et de faire évoluer les sous-espaces d'état définis par $\tilde{x}_{1}(t)$ et $\tilde{x}_{2}(t)$ de sorte à extraire un sous-système insensible à $d(t)$ à l'aide d'une injection de sortie.

Les méthodes développées dans les sections suivantes expriment le sous-espace d'état $\tilde{x}_{1}(t)$ à l'aide d'injections de sortie différentes. La première est issue d'une méthode analytique alors que la deuxième est déterminée à partir d'une méthode géométrique.

\subsubsection{Méthode analytique}

Cette méthode est principalement basée sur le travail de E. Alcorta Garcia et P.M. Frank amorcée dans [2], finalisée dans [3] et appliquée dans [60]. Elle s'appuie sur la théorie de la commande des systèmes non linéaires ([37], [56] et [63]) et prend en considération le modèle (1.15) où $d(t) \in \mathbb{R}^{s}$ est l'entrée inconnue dont les effets sont indésirables. De plus $s$ est supposé strictement inférieur à $m$, et satisfait à l'équation $\operatorname{dim}\left(\operatorname{span}\left\{D_{x}\right\}\right)=s, \forall(x(t), u(t))$.

Définition 1.4 [25] L'indice caractéristique d'ordre $i$ d'un modèle affine en la commande (1.5), relativement à l'ouvert $V$, noté $\rho_{i},(i=\{1, \cdots, p\})$ est l'unique entier défini par

$$
\rho_{i}=\min \left\{k \in \mathbb{N} \mid \exists j \in\{1, \cdots, m\}: L_{f_{j}} L_{f_{0}}^{k} h_{i}(x(t)) \neq 0, \forall x(t) \in V\right\}
$$


avec la convention $\rho_{i} \rightarrow+\infty$ si $L_{f_{j}} L_{f_{0}}^{k} h_{i} \equiv 0$ pour tout $j=1, \cdots$, m et tout $k \geq 0$ et où $L$ est l'opérateur de Lie défini en annexe A équation A.2.

D’une manière simple, nous pouvons dire que $\rho_{i}$ traduit le nombre de dérivations nécessaires pour voir apparaître le signal de commande dans l'écriture de la $i^{\mathrm{e}}$ sortie.

En référence à la définition 1.4, il nous a semblé plus simple de définir un indice caractéristique modifié de la manière suivante.

Définition 1.5 L'indice caractéristique modifié d'ordre $i$ associé au signal $d_{j}(t)$, relativement à l'ouvert $V$, noté $\rho_{m i}^{d_{j}}, i=1, \cdots, p$ est l'unique entier défini par

$$
\rho_{m i}^{d_{j}}=\min \left\{k \in \mathbb{N} \mid \exists \frac{\partial y_{x i}^{(k)}(t)}{\partial d_{j}(t)} \neq 0, \forall x(t) \in V\right\}
$$

avec $y_{x i}^{(k)}(t)$ la dérivée d'ordre $k$ de la $i^{e}$ sortie par rapport au temps et la convention identique à la définition $1.4 \rho_{m i}^{d_{j}} \rightarrow+\infty$ si $\frac{\partial y_{x i}^{(k)}(t)}{\partial d_{j}(t)} \equiv 0$ pour tout $k \geq 0$.

De la même manière que précédemment $\rho_{m i}^{d_{j}}$ traduit le nombre de dérivations nécessaires à l'apparition du signal $d_{j}(t)$ dans l'écriture de la $i$ e sortie. Par la suite nous associons à $\rho_{m}^{d_{j}}$ le plus petit indice caractéristique modifié solution de :

$$
\rho_{m}^{d_{j}}=\min _{i}\left(\rho_{m i}^{d_{j}}\right)
$$

Nous rappelons que lors de la définition du modèle (1.15) $d(t)=\left(d_{1}(t) \cdots d_{k}(t) \cdots d_{s}(t)\right)^{T}$ tel que $\forall k \in\{1, \cdots, s\} \operatorname{dim}\left(d_{k}(t)\right)=1$.

Le découplage du signal $d(t)$, s'effectue à l'aide d'une récurrence. Nous allons, dans un premier temps, chercher un sous-système insensible à une première composante de la perturbation puis à partir de celui-ci réitérer l'opération pour une seconde composante de la perturbation et ainsi de suite ...

Cette récurrence s'arrête à l'obtention du $s^{\mathrm{e}}$ sous-système insensible qui s'avère être insensible au signal $d(t)$.

Dans ce qui suit, nous décrivons les différentes étapes qui constituent la récurrence.

$\underline{\text { Premier pas : }}$

La composante de la perturbation notée $d_{k 1}(t)$ possédant le plus petit indice caractéristique modifié est sélectionnée $\left(k 1=\min _{j}\left(\rho_{m}^{d_{j}}\right)<+\infty\right)$. A ce coefficient $k 1$ est associée une sortie du système notée $y_{x d_{k 1}}(t)$ parmi le vecteur sortie $y_{x}(t)$.

Le modèle (1.15) se récrit :

$$
\Sigma_{N L}:\left\{\begin{array}{l}
\dot{x}(t)=f(x(t), u(t))+D_{x d_{k 1}}(x(t), u(t)) d_{k 1}(t)+\overline{D_{x d_{k 1}}}(x(t), u(t)) \overline{d_{k 1}}(t) \\
y_{x}(t)=h(x(t))
\end{array}\right.
$$


avec $\overline{d_{k 1}}(t)=\left(\begin{array}{llllll}d_{1}(t) & \cdots & d_{k 1-1}(t) & d_{k 1+1}(t) & \cdots & d_{s}(t)\end{array}\right)^{T}$ et $\overline{D_{x d_{k 1}}}(x(t), u(t))$ la matrice de pondération correspondante.

D'après [37], il est toujours possible de trouver une fonction vectorielle non linéaire $\phi_{d_{k 1}}(x(t), u(t))$ vérifiant les conditions suivantes :

$(i) \operatorname{rang}\left(\frac{\partial}{\partial x}\left(\begin{array}{c}y_{x d_{k 1}}(t) \\ \vdots \\ y_{x d_{k 1}}^{\left(\rho_{m} d_{k 1}-1\right)}(t) \\ \phi_{d_{k 1}}(x(t), u(t))\end{array}\right)\right)=n, \forall(x(t), u(t))$

(ii) $\frac{d}{d t} \phi_{d_{k 1}}(x(t), u(t))$ indépendant de $d_{k 1}(t)$

avec $\rho_{m}^{d_{k 1}}$ défini équation (1.18) (où $j=k 1$ ).

Il est alors possible de définir une transformation d'état $\tilde{x}^{1}(t)=\Phi_{d_{k 1}}(x(t), u(t))$ telle que :

$$
\tilde{x}^{1}(t)=\Phi_{d_{k 1}}(x(t), u(t))=\left(\begin{array}{c}
y_{x d_{k 1}}(t) \\
\vdots \\
y_{x d_{k 1}}^{\left(d_{k 1}-1\right)}(t) \\
----- \\
\phi_{d_{k 1}}(x(t), u(t))
\end{array}\right)=\left(\begin{array}{c}
\tilde{x}_{p 1}^{1}(t) \\
\vdots \\
\tilde{x}_{p \rho_{k} 1}^{1}(t) \\
----- \\
\tilde{x}_{\phi_{d_{k 1}}}^{1}(t)
\end{array}\right)=\left(\begin{array}{c}
\tilde{x}_{p}^{1}(t) \\
----- \\
\tilde{x}_{\phi_{d_{k 1}}}^{1}(t)
\end{array}\right)
$$

$\operatorname{avec} \operatorname{dim}\left(\tilde{x}_{p}^{1}(t)\right)=\rho_{m}^{d_{k 1}}<+\infty$

Par définition, cette transformation est une transformation d'état appelée difféomorphisme ( $c f$. annexe A définition A.2). Le modèle non linéaire s'écrit alors dans ce nouveau système de coordonnées ( $c f$. annexe B équation B.6) de la manière suivante :

$$
\Sigma_{\widetilde{N L}}:\left\{\begin{array}{l}
\dot{\tilde{x}}_{p 1}^{1}(t)=\tilde{x}_{p 2}^{1}(t) \\
\dot{\tilde{x}}_{p 2}^{1}(t)=\tilde{x}_{p 3}^{1}(t) \\
\vdots \\
\dot{\tilde{x}}_{p \rho_{m}^{d_{k 1}-1}}^{1}(t)=\tilde{x}_{p d_{k 1}}^{1}(t) \\
\dot{\tilde{x}}_{p \rho_{m}^{d_{k 1}}}^{1}(t)=L_{f}^{\rho_{m k}^{d_{k 1}}} h(x(t))+L_{D_{x d_{k 1}}} L_{f}^{\rho_{m}^{d_{k 1}}-1} h(x(t)) d_{k 1}(t)+\mathcal{O}\left(\overline{d_{k 1}}(t)\right) \\
----------------------------------1(t)) \\
\dot{\tilde{x}}_{\phi_{d_{k 1}}}^{1}(t)=\frac{d}{d t} \phi_{d_{k 1}}(x(t), u \\
y_{x}(t)=h \circ \Phi^{-1}\left(\tilde{x}^{1}(t), u(t)\right)
\end{array}\right.
$$


où $\mathcal{O}\left(\overline{d_{k 1}}(t)\right)$ est une fonction non linéaire de $x(t), u(t)$ et $d(t)$. Quant à $\frac{d}{d t} \phi_{d_{k 1}}(x(t), u(t))$, il s'agit d'une fonction non linéaire de $\tilde{x}^{1}(t), u(t), \dot{u}(t)$ et $\overline{d_{k 1}}(t)$.

Ainsi, l'écriture du système (1.21) est à comparer à l'écriture du système linéaire (1.11). En effet, la partie de l'état $\tilde{x}_{p}^{1}(t)$ est, d'une façon triviale, sensible à la perturbation $d_{k 1}(t)$.

Suite à cette transformation, il est possible d'extraire de (1.21) un sous-système insensible à $d_{k 1}(t)$. Celui-ci résulte d'une diminution de dimension et s'écrit :

$$
\Sigma_{N \widetilde{\text { Lréduit }}}^{1}:\left\{\begin{array}{l}
\dot{\tilde{x}}_{\phi_{d_{k 1}}}^{1}(t)=f_{d_{k 1}}\left(\tilde{x}_{p}^{1}(t), \tilde{x}_{\phi_{d_{k 1}}}^{1}(t), u(t), \dot{u}(t)\right)+\overline{D_{x d_{k 1}}}\left(\tilde{x}_{p}^{1}(t), \tilde{x}_{\phi_{d_{k 1}}}^{1}(t), u(t) \overline{\bar{d}_{k 1}}(t)\right. \\
\overline{y_{x d_{k 1}}}(t)=h_{d_{k 1}}\left(\tilde{x}^{1}, u(t)\right)
\end{array}\right.
$$

avec $\overline{y_{x d_{k 1}}}(t)$ l'ensemble des sorties privées de $y_{x d_{k 1}}(t)$ soit :

$$
\overline{y_{x d_{k 1}}}(t)=\left(\begin{array}{llllll}
y_{x 1}(t) & \cdots & y_{x d_{k 1}-1}(t) & y_{x d_{k 1}+1}(t) & \cdots & y_{x p}(t)
\end{array}\right)^{T}
$$

sachant que $\tilde{x}_{p}^{1}(t)$ s'identifie au vecteur défini par les sorties $y_{x d_{k 1}}(t)$ et ses $\rho_{m}^{d_{k 1}}-1$ dérivées. Nous pouvons modifier les deux fonctions vectorielles non linéaires $f_{d_{k 1}}(\bullet)$ et $\overline{D_{x d_{k 1}}}(\bullet)$ de sorte à obtenir deux nouvelles fonctions $\tilde{f}_{d_{k 1}}\left(\tilde{x}_{\phi_{d_{k 1}}}^{1}(t), u(t), \dot{u}(t)\right)$ et $\tilde{D}_{x d_{k 1}}\left(\tilde{x}_{\phi_{d_{k 1}}}^{1}(t), u(t)\right)$ synthétisées à l'aide d'une injection de sortie, c'est-à-dire :

$$
\begin{aligned}
f_{d_{k 1}}\left(\tilde{x}_{p}^{1}(t), \tilde{x}_{\phi_{d_{k 1}}}(t), u(t), \dot{u}(t)\right)= & \tilde{f}_{d_{k 1}}\left(\tilde{x}_{\phi_{d_{k 1}}}^{1}(t), u(t), \dot{u}(t)\right) \\
& +\tilde{\Psi}_{f_{d_{k 1}}}\left(y_{x d_{k 1}}(t), \cdots, y_{x d_{k 1}}^{\left(\rho_{m}^{d_{k 1}}-1\right)}(t), \tilde{x}_{\phi_{d_{k 1}}}^{1}(t), u(t), \dot{u}(t)\right) \\
& \Downarrow \\
\tilde{\Psi}_{f_{d_{k 1}}}(\bullet) & =f_{d_{k 1}}(\bullet)-\tilde{f}_{d_{k 1}}(\bullet) \\
\overline{D_{x d_{k 1}}}\left(\tilde{x}_{p}^{1}(t), \tilde{x}_{\phi_{d_{k 1}}}(t), u(t)\right)= & \tilde{D}_{x d_{k 1}}\left(\tilde{x}_{\phi_{d_{k 1}}}^{1}(t), u(t)\right) \\
& +\tilde{\Psi}_{D_{x d_{k 1}}}\left(y_{x d_{k 1}}(t), \cdots, y_{x d_{k 1}}^{\left(\rho_{k 1}^{d_{k 1}}-1\right)}(t), \tilde{x}_{\phi_{d_{k 1}}}^{1}(t), u(t)\right) \\
& \Downarrow \\
\tilde{\Psi}_{D_{x d_{k 1}}}(\bullet) & =\overline{D_{x d_{k 1}}}(\bullet)-\tilde{D}_{x d_{k 1}}(\bullet)
\end{aligned}
$$

Par construction, nous savons qu'il est possible de déterminer les injections de sorties vérifiant ces deux égalités (1.24) et (1.25). Ainsi, le sous-système (1.22) et son injection de sortie s'écrivent comme suit :

$$
\Sigma_{N \widetilde{\text { Lréduit }}}^{1}:\left\{\begin{aligned}
\dot{\tilde{x}}_{\phi_{d_{1}}}^{1}(t)= & \left\{\tilde{f}_{d_{k 1}}\left(\tilde{x}_{\phi_{d_{k 1}}}^{1}(t), u(t), \dot{u}(t)\right)\right. \\
& \left.+\tilde{\Psi}_{f_{d_{k 1}}}\left(y_{x d_{k 1}}(t), \cdots, y_{x d_{k 1}}^{\left(\rho_{m}^{d_{k 1}}-1\right)}(t), \tilde{x}_{\phi_{d_{k 1}}}(t), u(t), \dot{u}(t)\right)\right\} \\
+ & \left\{\tilde{D}_{x d_{k 1}}\left(\tilde{x}_{\phi_{d_{k 1}}}^{1}(t), u(t)\right) \overline{d_{k 1}}(t)\right. \\
& \left.+\tilde{\Psi}_{D_{x d_{k 1}}}\left(y_{x d_{k 1}}(t), \cdots, y_{x d_{k 1}}^{\left(\rho_{k 1}^{d_{k 1}}-1\right)}(t), \tilde{x}_{\phi_{d_{k 1}}}^{1}(t), u(t)\right) \overline{d_{k 1}}(t)\right\} \\
\frac{y_{x d_{k 1}}}{}(t)= & h_{d_{k 1}}\left(\tilde{x}^{1}(t), u(t)\right)
\end{aligned}\right.
$$


Il nous paraît important de faire le lien entre l'écriture du système précédent et celui obtenu lors de l'analyse d'un système linéaire (1.13). En effet, lors du premier pas (découplage d'une composante du signal perturbateur) nous retrouvons les différentes étapes mises en évidence dans le paragraphe 1.2.2.1. La première est un changement de coordonnées pour représenter l'équation d'état. Qui, dans cette approche particulière assure aussi la sélection d'une sortie. Enfin, l'expression des états sensibles à la perturbation grâce aux sorties et commandes (rôle de l'injection de sortie).

$\underline{i^{\mathrm{e}} \text { pas : }}$

A partir du sous-système précédent assurant le découplage d'une partie de l'état de la $(i-1)^{\mathrm{e}}$ composante de la perturbation $d(t)$ qui s'écrit :

$$
\Sigma_{N \widetilde{\text { Lréduit }}}^{i-1}:\left\{\begin{aligned}
\dot{\tilde{x}}_{\phi_{d_{k i-1}}^{i-1}}(t) & =f_{d_{k i-1}}\left(\tilde{x}_{p}^{i-1}(t), \tilde{x}_{\phi_{d_{k i-1}}^{i-1}}(t), u(t), \dot{u}(t)\right) \\
& +\overline{D_{x d_{k i-1}}}\left(\tilde{x}_{p}^{i-1}(t), \tilde{x}_{\phi_{d_{k i-1}}-1}(t), u(t)\right) \overline{d_{k i-1}}(t) \\
\overline{y_{x d_{k i-1}}}(t) & =h_{d_{k i-1}}\left(\tilde{x}^{i-1}(t), u(t)\right)
\end{aligned}\right.
$$

nous réalisons le $i^{\mathrm{e}}$ découplage. Nous précisons qu'à ce stade, le vecteur perturbation $\overline{d_{k i-1}}(t)$ n'est plus composé que de $s-i+1$ composantes. Parmi ces composantes, celle dont l'indice caractéristique modifié $\left(k i=\min _{j}\left(\rho_{m}^{d_{j}}\right)<+\infty\right)$ est le plus petit est sélectionnée et est associée à la sortie $y_{x d_{k i}}(t)$.

Ainsi, d'une manière analogue au premier pas (1.20), nous définissons le difféomorphisme suivant :

$$
\tilde{x}^{i}(t)=\Phi_{d_{k i}}(x(t), u(t))=\left(\begin{array}{c}
y_{x d_{k i}}(t) \\
\vdots \\
y_{x d_{k i}}^{\left(\rho_{m i}-1\right)}(t) \\
----- \\
\phi_{d_{k i}}(x(t), u(t))
\end{array}\right)
$$

Il est alors possible d'extraire un sous-système d'une forme similaire à celle obtenue après le $(i-1)^{\mathrm{e}}$ pas $(1.27)$ :

$$
\Sigma_{\text {NLéduit }}^{i}:\left\{\begin{aligned}
\dot{\tilde{x}}_{\phi_{d_{k i}}}^{i}(t) & =f_{d_{k i}}\left(\tilde{x}_{p}^{i}(t), \tilde{x}_{\phi_{d_{k i}}}^{i}(t), u(t), \dot{u}(t)\right) \\
& +\overline{D_{x d_{k i}}}\left(\tilde{x}_{p}^{i}(t), \tilde{x}_{\phi_{d_{k i}}}^{i}(t), u(t)\right) \overline{d_{k i}}(t) \\
\overline{y_{x d_{k i}}}(t) & =h_{d_{k i}}\left(\tilde{x}^{i}(t), u(t)\right)
\end{aligned}\right.
$$

Cependant l'intérêt principal est la possibilité d'écrire à l'aide d'injections de sortie, comme nous l'avons défini au premier pas (équations (1.24) et (1.25)), toute expression de $\tilde{x}_{p}^{i}(t)$ au sein du sous-système (1.29). 
Pour découpler le signal perturbateur $d(t)$ dans sa totalité, il nous faut effectuer $s$ itérations. Dans le cas où une solution existe, un sous-système de dimension $n-\sum_{i=1}^{s} \rho_{m}^{d_{k i}} \geq$ $\operatorname{dim}\left(\operatorname{span}\left\{D_{x}\right\}\right)$ (par hypothèse $\left.\operatorname{dim}\left(\operatorname{span}\left\{D_{x}\right\}\right)=s\right)$ insensible à $d(t)$ est représenté par ce qui suit :

$$
\Sigma_{N \text { Linsensible }}:\left\{\begin{array}{l}
\dot{\tilde{x}}_{\phi_{d_{s}}}(t)=\left\{\tilde{f}_{d_{s}}\left(\tilde{x}_{\phi_{d_{s}}}(t), U(t)\right)+\tilde{\Psi}_{f_{d_{s}}}\left(Y_{x}(t), \tilde{x}_{\phi_{d_{s}}}(t), U(t)\right)\right\} \\
\overline{y_{x d_{s}}}(t)=h_{d_{s}}(\tilde{x}(t))
\end{array}\right.
$$

où $Y_{x}(t)$ et $U(t)$ représentent respectivement les $\left(\rho_{m}^{d_{i}}-1\right)$ dérivées des sorties $y_{x d_{i}}(t)(i \in$ $1, \cdots, s)$ et les dérivées successives de $u(t)$.

Notons que si $\operatorname{dim}(d(t))=1$ alors l'injection de sortie se réduit à :

$$
\tilde{\Psi} \bullet\left(y_{x d}(t), \cdots, y_{x d}^{\left(\rho_{m}^{d}-1\right)}(t), \tilde{x}_{\phi_{d}}(t), u(t)\right)
$$

l'objet de cette remarque est d'insister sur la manière dont les sorties sont utilisées lors de la synthèse de l'injection. Ceci est un moyen de distinction entre les méthodes exposées et développées plus tard.

Cette méthode possède comme nous venons de le voir une mise en oeuvre relativement difficile de par sa procédure analytique itérative. Sans compter qu'il est nécessaire d'appliquer un nombre relativement grand (fonction de la dimension du signal $d(t)$ ) de difféomorphismes pour le découplage. Pour ces raisons, nous présentons, dans la section suivante une autre méthode sur la base d'outils géométriques.

\subsubsection{Méthode géométrique}

Au début des années 60, l'application d'outils mathématiques en automatique, constitue certainement l'origine d'une nouvelle approche qualifiée de géométrique. Nous retenons en particulier les travaux [65] et [51] qui font de nos jours référence dans ce domaine.

La première extension de ces méthodes mathématiques est publiée par A. Isidori ([38]) dans un article traitant des problèmes liés à la commande des systèmes non linéaires.

Dans cette section, nous nous attachons à présenter les travaux de C. De Persis et A. Isidori ([13], [14], [17]) visant principalement à donner des conditions d'existence d'un sous-système insensible à un signal $d(t)$. Ces travaux sont en lien direct avec le diagnostic de système et ces conditions sont liées à l'existence d'une injection de sortie. D'une manière analogue, nous faisons référence aux travaux de H. Hammouri et al ([33], [34] et [35]).

Les modèles considérés sont de la forme suivante :

$$
\Sigma_{N L}:\left\{\begin{array}{l}
\dot{x}(t)=f_{0}(x(t))+\sum_{i=1}^{m} f_{i}(x(t)) u_{i}(t)+D_{x}(x(t)) d(t) \\
y_{x}(t)=h(x(t))
\end{array}\right.
$$


avec $x(t) \in \mathbb{R}^{n}, u(t) \in \mathbb{R}^{m}, d(t) \in \mathbb{R}^{s}$ et $y_{x}(t) \in \mathbb{R}^{p}$ et sous l'hypothèse $\operatorname{dim}\left(\operatorname{span}\left\{D_{x}\right\}\right)=s<p, \forall x(t)$.

La première étape est la détermination de la plus petite distribution invariante pour la dynamique du système $\left(\left(f_{i}\right)\right.$-invariante $\forall i \in\{0, \cdots, m\}$, cf. annexe $\left.\mathrm{B}\right)$, via une injection de sortie, contenant $\operatorname{span}\left\{D_{x}\right\}$. Il s'agit des états (ou d'une combinaison de variables d'état) ne pouvant pas s'écrire à l'aide des sorties sans la connaissance de la perturbation. Cette combinaison d'états nommée sous-espace d'état par la suite, est déterminée à l'aide de la séquence non-décroissante suivante :

$$
\left\{\begin{array}{l}
S_{0}^{D_{x}}=\operatorname{span}\left\{D_{x}\right\} \\
S_{i+1}^{D_{x}}=\bar{S}_{i}^{D_{x}}+\sum_{k=0}^{m}\left[f_{k}, \bar{S}_{i}^{D_{x}} \cap \operatorname{ker}\{d h\}\right]
\end{array}\right.
$$

où $[$,$] représente les crochets de Lie ( c f$. annexe A équation A.6), $\bar{S}$ la plus petite distribution involutive contenant la distribution $S$ (annexe A définition A.1) et $d h$ la matrice jacobienne de $h(x(t))$ (cf. annexe A équation A.1).

Une condition d'arrêt à la séquence précédente est donnée par :

$$
\bar{S}_{i}^{D_{x}}=S_{i+1}^{D_{x}} \Rightarrow S_{*}^{D_{x}}=\bar{S}_{i}^{D_{x}}
$$

Remarquons que dans le pire des cas $\left(\operatorname{dim}\left(\operatorname{span}\left\{S_{i}^{D_{x}}\right\}\right)=n\right)$, la distribution $S_{*}^{D_{x}}$ représente l'espace d'état dans sa totalité. Cette propriété est synonyme d'une perturbation se propageant dans tout l'espace d'état sans qu'il soit possible de la découpler d'un sous-espace d'état.

A titre indicatif, pour des raisons de commodité, il est parfois plus intéressant d'utiliser les co-distributions (espace dual des distributions $\square$ noté $\square^{\star}$ ).

A ce titre, la séquence non-décroissante de co-distributions suivante est développée :

$$
\left\{\begin{array}{l}
Q_{0}^{D_{x}}=\Theta \cap \operatorname{span}\{d h\} \\
Q_{i+1}^{D_{x}}=\Theta \cap\left(\sum_{k=0}^{m} L_{f_{k}} Q_{i}+\operatorname{span}\{d h\}\right)
\end{array}\right.
$$

Pour plus de précisions, la démonstration de l'équivalence est donnée dans [14].

Dans la suite de ce document, nous utilisons principalement la première séquence nondécroissante (1.33). Lors de la synthèse du sous-espace d'état $S_{*}^{D_{x}}$, il est primordial de lier les éléments qui composent la séquence non décroissante à l'expression de l'injection de sortie. En effet, le découplage proposé par C. De Persis et A. Isidori utilise une injection de sortie du type $\Psi\left(x(t), u(t), y_{x}(t)\right)$. Or, le sous-espace d'état reconstruit par une telle injection de sortie est limité par une "simple" utilisation de " $y_{x}(t)$ ", ce qui se traduit par l'intersection $\bar{S}_{i}^{D_{x}} \cap \operatorname{ker}\{d h\}$. 
Les conditions d'existence d'un sous-système découplé s'arrêtent à la non nullité du sousespace d'état orthogonal à $S_{*}^{D_{x}}$ soit $\left(S_{*}^{D_{x}}\right)^{\perp} \neq\{0\}$.

En revanche, lors de la génération de résidus pour le diagnostic, les sous-espaces d'états insensibles à des défauts doivent non seulement être non nuls mais aussi posséder certaines propriétés supplémentaires (ceci sera amplement développé dans le chapitre 3).

Ainsi, si $\left(S_{*}^{D_{x}}\right)^{\perp} \neq\{0\}$, il est possible, par un simple changement de coordonnées $\tilde{x}(t)=$ $\Phi(x(t))$, d'écrire le système (1.32) sous la forme suivante :

$$
\left\{\begin{aligned}
\dot{\tilde{x}}_{1}(t)= & \tilde{f}_{0,1}\left(\tilde{x}_{1}(t), \tilde{x}_{2}(t)\right)+\sum_{i=1}^{m} \tilde{f}_{i, 1}\left(\tilde{x}_{1}(t), \tilde{x}_{2}(t)\right) u_{i}(t)+\tilde{D}_{x}\left(\tilde{x}_{1}(t), \tilde{x}_{2}(t)\right) d(t) \\
\dot{\tilde{x}}_{2}(t)= & \left\{\tilde{f}_{0,2}\left(\tilde{x}_{1}(t), \tilde{x}_{2}(t)\right)-\tilde{\Psi}_{0}\left(\tilde{x}_{2}(t), y_{x}(t)\right)\right\}+\sum_{i=1}^{m}\left\{\tilde{f}_{i, 2}\left(\tilde{x}_{1}(t), \tilde{x}_{2}(t)\right)-\tilde{\Psi}_{i}\left(\tilde{x}_{2}(t), y_{x}(t)\right)\right\} u_{i}(t) \\
& +\tilde{\Psi}_{0}\left(\tilde{x}_{2}(t), y_{x}(t)\right)+\sum_{i=1}^{m} \tilde{\Psi}_{i}\left(\tilde{x}_{2}(t), y_{x}(t)\right) u_{i}(t) \\
y_{x}(t)= & \tilde{h}\left(\tilde{x}_{1}(t), \tilde{x}_{2}(t)\right)
\end{aligned}\right.
$$

où $\tilde{\Psi}_{0}\left(\tilde{x}_{2}(t), y_{x}(t)\right)+\sum_{i=1}^{m} \tilde{\Psi}_{i}\left(\tilde{x}_{2}(t), y_{x}(t)\right) u_{i}(t)=\tilde{\Psi}\left(\left(\tilde{x}_{2}(t), u(t), y_{x}(t)\right)\right)$ représente l'injection de sortie précédemment introduite, exprimée dans une nouvelle base de coordonnées ( $c f$. annexe B équation B.6). Tout l'intérêt d'une telle injection est mis en relief par l'égalité :

$$
\tilde{f}_{i, 2}\left(\tilde{x}_{1}(t), \tilde{x}_{2}(t)\right)-\tilde{\Psi}_{i}\left(\tilde{x}_{2}(t), y_{x}(t)\right)=\breve{f}_{i, 2}\left(\tilde{x}_{2}(t)\right), \forall i \in\{0, \cdots, m\}
$$

C'est-à-dire qu'à l'aide de l'injection de sortie, nous exprimons au sein de l'écriture de $\dot{\tilde{x}}_{2}(t)$ toute contribution du sous-espace d'état sensible à la perturbation $\tilde{x}_{1}(t)$.

Suite aux simplifications d'écriture, le système (1.36) devient :

$$
\left\{\begin{array}{l}
\dot{\tilde{x}}_{1}(t)=\tilde{f}_{0,1}\left(\tilde{x}_{1}(t), \tilde{x}_{2}(t)\right)+\sum_{i=1}^{m} \tilde{f}_{i, 1}\left(\tilde{x}_{1}(t), \tilde{x}_{2}(t)\right) u_{i}(t)+\tilde{D}_{x}\left(\tilde{x}_{1}(t), \tilde{x}_{2}(t)\right) d(t) \\
\dot{\tilde{x}}_{2}(t)=\breve{f}_{0,2}\left(\tilde{x}_{2}(t)\right)+\sum_{i=1}^{m} \breve{f}_{i, 2}\left(\tilde{x}_{2}(t)\right) u_{i}(t)+\tilde{\Psi}_{0}\left(\tilde{x}_{2}(t), y_{x}(t)\right)+\sum_{i=1}^{m} \tilde{\Psi}_{i}\left(\tilde{x}_{2}(t), y_{x}(t)\right) u_{i}(t) \\
y_{x}(t)=\tilde{h}\left(\tilde{x}_{1}(t), \tilde{x}_{2}(t)\right)
\end{array}\right.
$$

Nous ajoutons que lors de nos différentes applications, il a semblé plus judicieux de déterminer l'injection de sortie suite au changement de coordonnées précédemment défini. En effet, le difféomorphisme $\tilde{x}(t)=\Phi(x(t))$ doit permettre l'écriture du système précédent et est directement lié à la distribution $\left(S_{*}^{D_{x}}\right)^{\perp}$. La distribution $\frac{\partial \phi(x(t))}{\partial x(t)}$ est choisie afin que $\Phi(x(t))$ 
possède les propriétés d'un difféomorphisme ( $c f$. annexe A définition A.2) :

$$
\frac{\partial \Phi(x(t))}{\partial x(t)}=\left(\begin{array}{c}
\frac{\partial \phi(x(t))}{\partial x(t)} \\
----- \\
\left(\left(S_{*}^{D_{x}}\right)^{\perp}\right)^{T}
\end{array}\right)
$$

où $\tilde{x}_{2}(t)$ évolue dans le sous-espace d'état involutif représenté par la distribution $\left(S_{*}^{D_{x}}\right)^{\perp}$.

Suite à la présentation des deux méthodes de découplage analytique et géométrique, nous comparons dans la section suivante les particularités de chacune d'elles.

\subsubsection{Comparaison des méthodes}

Nous rappelons les conditions d'existence d'un filtre découplant l'effet d'une perturbation $d(t)$ de dimension 1 suite à l'application des méthodes analytiques et géométriques. La dimension du sous-espace d'état insensible à une perturbation est ensuite évaluée. Les deux méthodes sont à distinguer tant du point de vue des résultats que de par les approches.

Approche analytique :

L'injection de sortie employée pour le découplage s'écrit comme suit :

$$
\tilde{\Psi}\left(\tilde{x}_{2}(t), u(t), \dot{u}(t), y_{x d}^{(0)}(t), \cdots, y_{x d}^{\left(\rho_{m}^{d}-1\right)}(t)\right)
$$

qui permet l'encadrement suivant :

$$
n-\rho_{m}^{d} \leq \operatorname{dim}(\text { sous-espace d'état insensible à la perturbation } d(t)) \leq n-1
$$

Approche géométrique :

Le découplage d'une partie des états de l'effet d'une perturbation est obtenu à l'aide d'une injection de sortie de la forme suivante :

$$
\tilde{\Psi}\left(\tilde{x}_{2}(t), u(t), y_{x}(t)\right)
$$

qui assure un encadrement du sous-espace d'état insensible à la perturbation suivant :

$0 \leq \operatorname{dim}\left(\left(S_{*}^{D_{x}}\right)^{\perp}\right)=\operatorname{dim}($ sous-espace d'état insensible à la perturbation $d(t)) \leq n-1$

où la borne inférieure de l'inégalité est fortement dépendante du sous-espace d'état représenté par $\operatorname{ker}\{d h\}$.

Ainsi, si la dimension du sous-espace d'état insensible à la perturbation $d(t)$ est strictement supérieure à 0 avec l'une ou l'autre des injections ((1.40) et (1.42)) alors il est possible 
de découpler la perturbation. En revanche, nous ne pouvons pas déterminer d'une manière générale quelle est la méthode donnant de meilleurs résultats (en terme de dimension du sous-espace d'état insensible à une perturbation).

Notons que les bornes supérieures des inégalités (1.41) et (1.43) sont directement liées à la dimension de la perturbation. Dans le chapitre 2 (sections 2.3 .2 et 2.3.3), nous nous attachons à accroître la dimension du sous-espace d'état insensible à la perturbation.

La détermination de l'injection de sortie à l'aide de l'une des méthodes précédentes ne constitue qu'une première étape menant à la génération de résidus.

\subsubsection{Génération de résidus}

Un résidu est un signal issu des relations de redondance analytique du modèle du système. Il a pour objectif de mettre en évidence, dans le cadre du diagnostic, l'apparition d'un ou plusieurs défauts.

Il existe de nombreux outils pour générer un résidu. Pour les systèmes non linéaires, les plus connus sont les suivants :

- observateurs ou filtres obtenus à l'aide de linéarisations à différents niveaux [53], [67], observateurs [8], filtres de Kalman [9], [61], [66]. Ces générateurs de résidus se caractérisent à l'aide d'une linéarisation soit de l'état soit de l'erreur d'estimation.

- observateurs ou filtres [26, 27], [33, 35], [14], [31], [45] et [4], synthétisés à l'aide de méthodes totalement non linéaires telles que celles présentées dans ce chapitre (méthode analytique ou géométrique) et basés sur l'étude des particularités structurelles du modèle du système.

- relations de parité [47], qui se fondent sur la génération d'équations de redondance indépendantes de l'état.

- estimation de paramètres [28], [62]. Il s'agit d'identifier en ligne certains paramètres du système et d'utiliser les résultats pour générer des résidus.

Une attention particulière est portée aux méthodes qui utilisent les filtres (ou observateurs) en tant qu'estimateurs d'états. A titre d'exemple, l'équation du filtre associé à la méthode géométrique précédemment développée, est donnée ci-dessous (1.44), avec $\tilde{z}_{2}(t)$ en relation directe avec $\left(S_{*}^{D_{x}}\right)^{\perp}$ défini en (1.39).

$$
\Sigma_{F N L}:\left\{\begin{aligned}
\dot{z}(t)=\quad & f_{0}(z(t))+\sum_{i=1}^{m} f_{i}(z(t)) u_{i}(t) \\
& +\left\{\Psi_{0}\left(\tilde{z}_{2}(t), y_{z}(t)\right)-\Psi_{0}\left(\tilde{z}_{2}(t), y_{x}(t)\right)\right\} \\
& +\sum_{i=1}^{m}\left\{\Psi_{i}\left(\tilde{z}_{2}(t), y_{z}(t)\right)-\Psi_{i}\left(\tilde{z}_{2}(t), y_{x}(t)\right)\right\} u_{i}(t) \\
y_{z}(t)= & h(z(t))
\end{aligned}\right.
$$

Les estimations $z(t)$ de l'état du système, nous permettent de générer des résidus dont l'équation générale est une fonction non linéaire des sorties estimées $\left(y_{z}(t)\right)$ et des sorties 
mesurées $\left(y_{x}(t)\right)$ :

$$
r(t)=\chi \circ h\left(y_{x}(t)\right)-\chi \circ h\left(y_{z}(t)\right)
$$

Dans le cadre de la localisation des défauts, il est nécessaire de synthétiser un nombre suffisant de résidus $(\operatorname{dim}(r(t)) \geq \operatorname{dim}(w(t)))$ pour différencier les défauts. Plusieurs contextes sont considérés et les contraintes quant à la génération de résidus, diminuent $(i)$ ou augmentent (ii) :

( $i)$ seul un défaut peut apparaître : les défauts doivent être la cause de résidus différents deux à deux,

(ii) plusieurs défauts peuvent apparaître en même temps : tous les résidus sont différents quel que soit le nombre de défauts présents.

Ces dernières considérations font l'objet des travaux exposées dans la section suivante.

\subsection{Structuration des résidus}

Suivant le nombre de filtres utilisés pour générer le vecteur résidu, deux types de structuration sont à distinguer :

- en présence d'un unique filtre, la génération d'un vecteur résidu constitué de composantes aux propriétés différentes (en terme de sensibilités aux défauts) est assurée,

- un banc de filtres est mis en oeuvre, chacun des filtres mis en parallèles possède des propriétés différentes de sensibilité aux défauts. Trois structures couramment employées (structures d'observateurs dédiés, simplifiés [29] et généralisés [12]) sont détaillées section 1.3.2.

Ces distinctions ne sont que trop rarement effectuées lors de l'analyse d'un système menant au diagnostic. Nous montrons dans ce document qu'un filtre unique peut offrir les même propriétés qu'un banc de filtres (en terme d'isolation des défauts).

\subsubsection{Le problème fondamental de la génération de résidus}

Pouvoir détecter et localiser tous les défauts, quel que soit l'ordre d'apparition, à l'aide d'un générateur de résidu (un filtre par exemple) est un problème bien connu portant le nom de problème fondamental de la génération de résidus (Fundamental Problem of Residual Generation, F.P.R.G. en anglais). Ce problème, a fait l'objet de nombreuses publications dans le cadre du diagnostic des systèmes linéaires ([7], [43], [64]) et plus récemment ([52] et [48]). En revanche, l'extension de ces travaux aux systèmes non linéaires est relativement récente ([35] et [17]). Ceci nécessite de construire un vecteur résidu $r(t)$ non seulement de 


\begin{tabular}{|c||c|c|c|c|c|}
\hline Résidus & $\begin{array}{c}\text { sans } \\
\text { défaut }\end{array}$ & $w_{1}(t)$ & $w_{2}(t)$ & $\cdots$ & $w_{q}(t)$ \\
\hline \hline$r_{1}(t)$ & insensible & sensible & insensible & $\cdots$ & insensible \\
\hline$r_{2}(t)$ & insensible & insensible & sensible & $\cdots$ & insensible \\
\hline$\vdots$ & $\vdots$ & $\vdots$ & $\vdots$ & $\ddots$ & $\vdots$ \\
\hline$r_{q}(t)$ & insensible & insensible & insensible & $\cdots$ & sensible \\
\hline
\end{tabular}

TAB. 1.1: Table de signature du F.P.R.G.

dimension égale au nombre de défauts mais aussi possédant la propriété suivante :

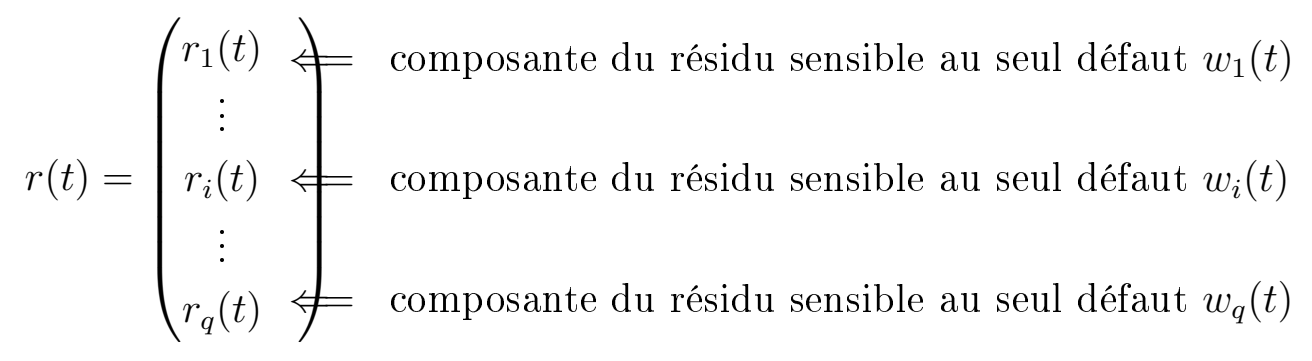

avec $q=\operatorname{dim}(w(t))$.

Les conditions d'existence d'un tel filtre sont rappelées au chapitre 3, mais nous pouvons d'ores et déjà préciser qu'elles sont très restrictives ([13]). En effet, le découplage "parfait" (1.46) est difficilement réalisable pour un système réel. Pour simplifier l'écriture de la sensibilité des résidus aux différents défauts nous utilisons des tables de signatures. La table de signatures TAB. 1.1 correspond au cas du F.P.R.G. (1.46).

Dans la suite de ce manuscrit, nous supposons le système parfaitement décrit par le modèle non linéaire, ainsi toute modification du résidu ne peut être due qu'à l'apparition d'un défaut ou d'une perturbation. En toute rigueur, le résidu doit être soumis à un test visant à détecter un changement de caractéristiques synonyme de l'apparition des défauts. A titre d'exemple, citons les techniques à base de calcul de seuils sur la moyenne, la variance, ... ([6]). Suite à cette étape d'évaluation, nous associons, de manière équivalente, un résidu non nul à l'apparition du défaut.

Une alternative aux contraintes liées à la construction d'un filtre résolvant le F.P.R.G., consiste à considérer un banc de filtres. Ainsi, les contraintes de construction ne se concentrent plus sur un unique filtre mais se répartissent sur l'ensemble des filtres.

\subsubsection{Structures particulières de banc d'observateurs}

Ces structures particulières sont principalement employées pour le diagnostic de systèmes linéaires ([58] et [59]), car elles s'appuient sur des notions très précises d'observabilité. Cependant, nous montrons au chapitre 3 que celles-ci peuvent s'étendre au diagnostic des systèmes 
non linéaires. Ainsi les méthodes présentées dans ce paragraphe ne distinguent ni le type de générateur de résidu (filtre ou observateur) ni le domaine d'application (linéaire ou non linéaire). Elles s'appliquent donc a fortiori avec les filtres suivants :

- filtres linéaires :

$$
\Sigma_{F L}:\left\{\begin{array}{l}
\dot{z}(t)=A z(t)+B u(t)+L\left(y_{z}(t)-y_{x}(t)\right) \\
y_{z}(t)=C z(t)
\end{array}\right.
$$

- filtres non linéaires :

$$
\Sigma_{F N L}:\left\{\begin{aligned}
\dot{z}(t)= & f_{0}(z(t))+\sum_{i=1}^{m} f_{i}(z(t)) u_{i}(t) \\
& +\left\{\Psi_{0}\left(z(t), y_{z}(t)\right)-\Psi_{0}\left(z(t), y_{x}(t)\right)\right\} \\
& +\sum_{i=1}^{m}\left\{\Psi_{i}\left(z(t), y_{z}(t)\right)-\Psi_{i}\left(z(t), y_{x}(t)\right)\right\} u_{i}(t) \\
y_{z}(t)= & h(z(t))
\end{aligned}\right.
$$

où $L(\bullet)$ et $\Psi_{i}(\bullet)(i=\{0, \cdots, m\})$ constituent les injections de sortie.

Dans les paragraphes suivants, les structures particulières de banc d'observateurs menant à bien l'isolation de tout défaut (actionneur, système, capteur) sont présentées. Nous nous aidons des résultats propres au découplage des systèmes linéaires et non linéaires respectivement développés dans [30], [65] et [27], [38]. Pour chacune des structures, nous développons la méthode de synthèse du banc d'observateurs, la logique de décision associée ainsi que les conditions suffisantes de construction des filtres dans le cadre de défauts capteur pour un système linéaire. Seul le cas des défauts capteur est étudié car l'indice caractéristique associé à chaque défaut est nul $\left(\rho_{m}^{w_{i}}=0\right)$ ce qui simplifie les conditions de construction du banc de filtres. En revanche, une étude approfondie considérant les particularités géométriques du système s'avère nécessaire si d'autres défauts sont considérés.

\subsubsection{Structure d'observateurs dédiés}

Dans la littérature : Dedicated Observer Scheme (D.O.S., figure 1.2).

Synthèse du banc: nous synthétisons autant d'observateurs que de défauts, chacun de ces observateurs permet la génération d'un résidu insensible à tous les défauts sauf un.

Logique de décision : l'occurrence du défaut est indiquée par l'observateur source de mauvaises estimations. Cet observateur n'est sensible qu'à un seul défaut et permet donc la détection et la localisation de ce dernier. 


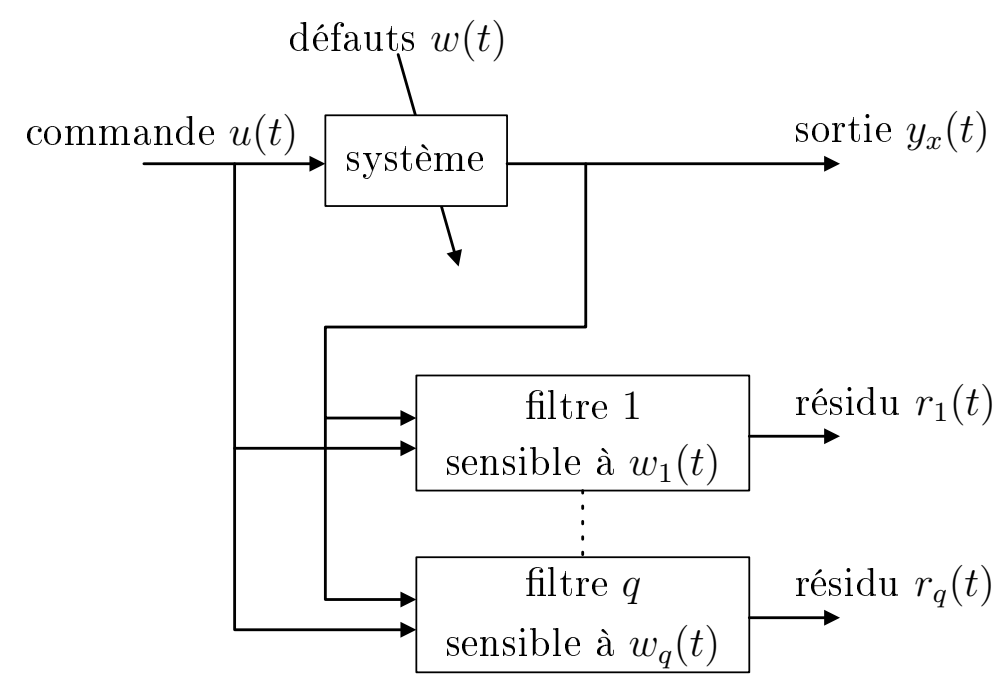

FIG. 1.2: Structure d'observateurs dédiés

Conditions suffisantes de construction (défauts capteur, cas linéaire) : la condition de construction est particulièrement difficile à satisfaire :

$$
\forall(\lambda, i) \text { il faut }: \operatorname{dim}\left(\operatorname{span}\left\{\begin{array}{c}
A-\lambda I \\
C_{i}
\end{array}\right\}\right)=n
$$

avec $C=\left(C_{1}^{T} \cdots C_{i}^{T} \cdots C_{p}^{T}\right)^{T}$.

\subsubsection{Structure d'observateur simplifié}

Dans la littérature : Simplified Observer Scheme (S.O.S., figure 1.3).

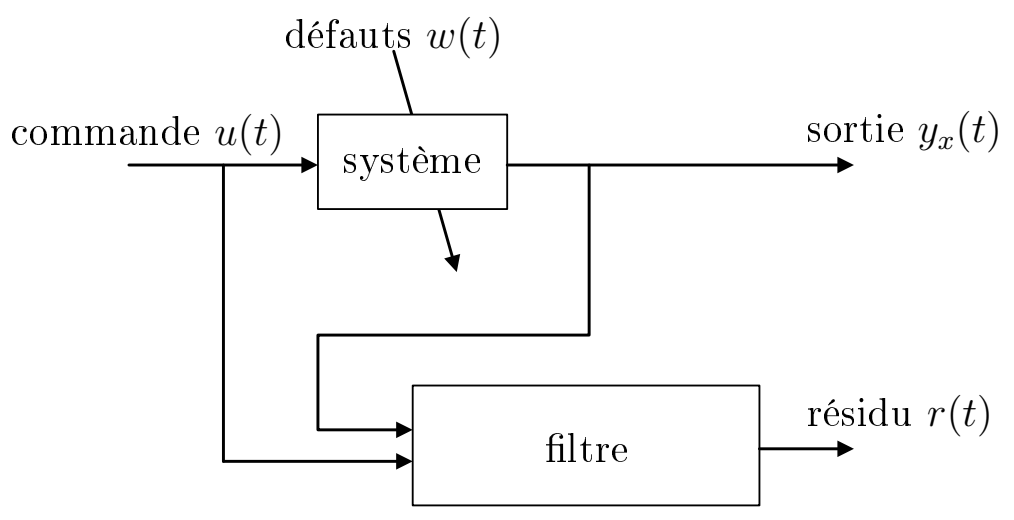

FIG. 1.3: Structure d'observateurs simplifiés

Synthèse du banc: seul un observateur constitue le banc. Il est synthétisé de sorte à n'être sensible qu'à un groupe de défauts. 
Logique de décision : si l'un des défauts auquel est sensible l'observateur apparaît alors les estimations sont erronées. Dans le cas contraire elles sont exactes.

Conditions suffisantes de construction (défauts capteur, cas linéaire) : celles-ci sont plus facile à vérifier que les précédentes :

$$
\forall(\lambda) \text { il existe } i \text { tel que }: \operatorname{dim}\left(\operatorname{span}\left\{\begin{array}{c}
A-\lambda I \\
C_{i}
\end{array}\right\}\right)=n
$$

\subsubsection{Structure d'observateurs généralisés}

Dans la littérature : Generalized Observer Scheme (G.O.S., figure 1.4).

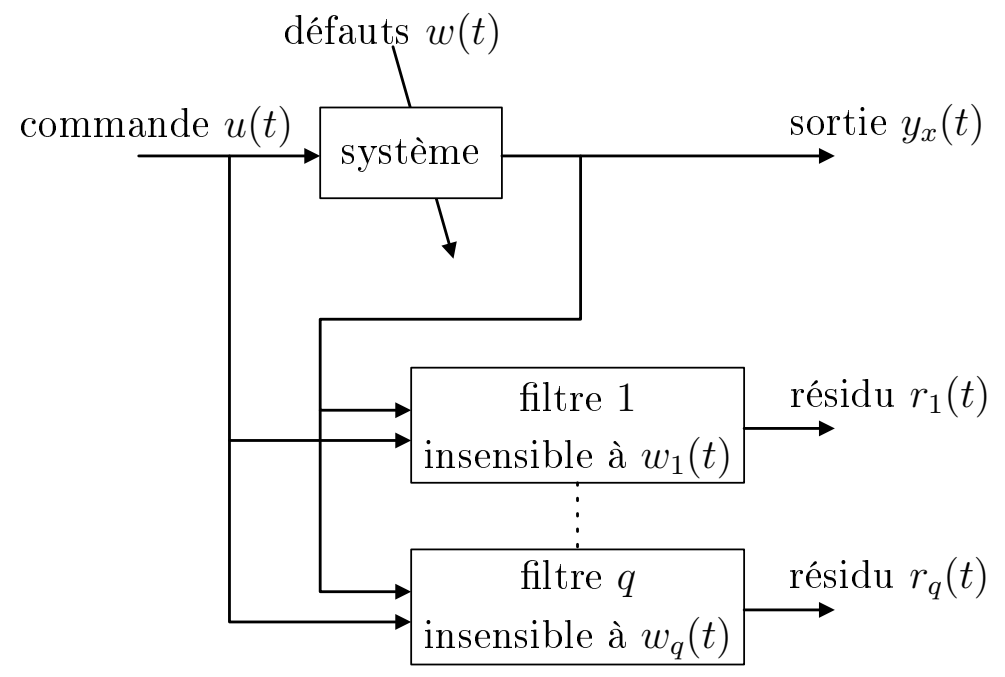

FIG. 1.4: Structure d'observateurs généralisés

Synthèse du banc: $q$ observateurs sont synthétisés, chacun d'eux est insensible à un seul défaut.

Logique de décision : si un défaut apparaît, l'estimation de l'ensemble du sous-espace d'état sensible à ce défaut est erronée si elle ne provient pas de l'observateur insensible à ce seul défaut.

Conditions suffisantes de construction (défauts capteur, cas linéaire) : les conditions de construction sont les plus faciles à vérifier comparativement aux deux précédentes :

$$
\forall(\lambda, i) \text { il faut }: \operatorname{dim}\left(\operatorname{span}\left\{\begin{array}{c}
A-\lambda I \\
\bar{C}_{i}
\end{array}\right\}\right)=n
$$


avec $\bar{C}_{i}=\left(C_{1}^{T} \cdots C_{i-1}^{T} C_{i+1}^{T} \cdots C_{p}^{T}\right)^{T}$.

Dans ce chapitre, nous avons présenté les travaux de références dans le cadre tant du découplage d'une partie de l'état aux perturbations que du diagnostic et la génération de résidus. Dans la section suivante, par rapport à l'ensemble de ces travaux nous positionnons les nôtres en nous restreignant à certaines hypothèses de travail.

\subsection{Conclusion et hypothèses de travail}

\subsubsection{Conclusion}

Afin de mettre en exergue l'intérêt de nos travaux, nous avons fait l'inventaire, dans ce chapitre, des différentes recherches effectuées dans le domaine du diagnostic des systèmes non linéaires. Les différents modèles non linéaires rencontrés dans la littérature ont été présentés. La modélisation des perturbations ou défauts y est associée car elle est le point de départ de toute démarche de diagnostic. La première approche (paragraphe 1.2.2.3), dont la complexité dépend des non linéarités traduisant le comportement du système, est une extension des méthodes de commande non linéaire. En revanche, l'approche géométrique semble plus générique, bien que demandant un effort particulier de formalisme.

Ensuite, la détection et la localisation des défauts sont effectuées à l'aide de résidus structurés. A ce titre, nous rappelons le F.P.R.G. qui constitue un problème de référence en diagnostic. Sans oublier les structures particulières d'observateurs (D.O.S., S.O.S. et G.O.S.) qui sont très employées pour le diagnostic des systèmes linéaires.

Les différents travaux présentés par la suite se positionnent à plusieurs niveaux.

Sur la base de ceux effectués par E. Alcorta Garcia, C. De Persis et A. Isidori, nous nous intéressons à la synthèse de filtres par injection de sortie. Afin de mettre en relief l'intérêt du travail réalisé par rapport aux méthodes précédemment citées, considérons l'exemple académique suivant. 


\section{Exemple académique}

Dans cet exemple nous étudions le système linéaire représenté par le modèle suivant :

$$
\Sigma_{L}:\left\{\begin{array}{l}
\dot{x}_{1}(t)=-x_{1}(t)+u_{1}(t)+d(t) \\
\dot{x}_{2}(t)=x_{1}(t)-x_{2}(t) \\
\dot{x}_{3}(t)=x_{2}(t)-x_{3}(t)+x_{4}(t)+u_{2}(t) \\
\dot{x}_{4}(t)=-x_{3}(t) \\
y_{x 1}(t)=x_{3}(t) \\
y_{x 2}(t)=x_{4}(t)
\end{array}\right.
$$

où $D_{x}=\left(\begin{array}{llll}1 & 0 & 0 & 0\end{array}\right)^{T}$.

Nous appliquons les méthodes présentées dans ce chapitre afin d'évaluer leurs performances. Nous débutons par la méthode linéaire se fondant sur deux découplages à partir de S.V.D.

\section{Méthode linéaire :}

Bien que la décomposition du vecteur qui pondére la perturbation $d(t)$ soit évidente $D_{x 1}=$ $\left(\begin{array}{llll}1 & 0 & 0 & 0\end{array}\right)^{T}$ (équation (1.11)), la matrice $C_{11}$ (1.12) est nulle. Il n'est donc pas possible de découpler la perturbation à l'aide de cette méthode.

Ainsi la dimension du sous-espace d'état insensible à la perturbation est réduite à 0 et la dimension du sous-espace d'état sensible est égale à 4 .

Nous concluons en ajoutant que ceci était prévisible car la matrice $C D_{x}=0$.

Dans ce qui suit nous appliquons les deux méthodes non linéaires citées auparavant (analytique paragraphe 1.2.2.3 et géométrique paragraphe 1.2.2.4).

Méthode analytique :

Suite aux dérivations successives des sorties, nous obtenons $\rho_{m}^{d}=\rho_{m 1}^{d}=3$ (défini par l'équation (1.18)). Ainsi le difféomorphisme (1.20) est construit à partir de la première sortie :

$$
\tilde{x}(t)=\Phi_{d}(x(t), u(t))=\left(\begin{array}{c}
y_{x 1}^{(0)}(t) \\
y_{x 1}^{(1)}(t) \\
y_{x 1}^{(2)}(t) \\
\phi(x(t), u(t))
\end{array}\right)=\left(\begin{array}{c}
x_{3}(t) \\
x_{2}(t)-x_{3}(t)+x_{4}(t)+u_{2}(t) \\
x_{1}(t)-2 x_{2}(t)-x_{4}(t)-u_{2}(t)+u_{2}^{(1)}(t) \\
x_{4}(t)
\end{array}\right)
$$


Dans ce système de coordonnées le modèle (1.52) s'écrit comme suit :

$$
\Sigma_{\widetilde{L}}:\left\{\begin{array}{l}
\dot{\tilde{x}}_{1}(t)=\tilde{x}_{2}(t) \\
\dot{\tilde{x}}_{2}(t)=\tilde{x}_{3}(t) \\
\dot{\tilde{x}}_{3}(t)=-3 \tilde{x}_{1}(t)-4 \tilde{x}_{2}(t)-3 \tilde{x}_{3}(t)+\tilde{x}_{4}(t)+u_{1}(t)+u_{2}(t)+2 u_{2}^{(1)}(t)+u_{2}^{(2)}(t)+d(t) \\
\dot{\tilde{x}}_{4}(t)=-\tilde{x}_{1}(t) \\
y_{x 1}(t)=\tilde{x}_{1}(t) \\
y_{x 2}(t)=\tilde{x}_{4}(t)
\end{array}\right.
$$

Nous pouvons alors synthétiser le filtre suivant :

$$
\Sigma_{\widetilde{F L}}:\left\{\begin{array}{l}
\dot{\tilde{z}}_{1}(t)=\tilde{z}_{2}(t) \\
\dot{\tilde{z}}_{2}(t)=\tilde{z}_{3}(t) \\
\dot{\tilde{z}}_{3}(t)=-3 \tilde{z}_{1}(t)-4 \tilde{z}_{2}(t)-3 \tilde{z}_{3}(t)+\tilde{z}_{4}(t)+u_{1}(t)+u_{2}(t)+2 u_{2}^{(1)}(t)+u_{2}^{(2)}(t) \\
\dot{\tilde{z}}_{4}(t)=-y_{x 1}(t) \\
y_{z 1}(t)=\tilde{z}_{1}(t) \\
y_{z 2}(t)=\tilde{z}_{4}(t)
\end{array}\right.
$$

avec l'injection de sortie suivante

$\tilde{\Psi}\left(\tilde{x}_{4}(t), u(t), u^{(1)}(t), y_{x 1}^{(0)}(t), y_{x 1}^{(1)}(t), y_{x 1}^{(2)}(t)\right)=\left(\begin{array}{llll}0 & 0 & 0 & -y_{x 1}(t)\end{array}\right)^{T}(c f$. équation (1.40)). Ainsi seul l'état estimé par $\tilde{z}_{4}(t)$ est insensible à la perturbation $d(t)$.

Alors la dimension du sous-espace d'état insensible à la perturbation est égale à 1 et la dimension du sous-espace d'état sensible est égale à 3 .

\section{Méthode géométrique :}

Sans développer les calculs, nous montrons que le plus petit sous-espace d'état sensible à un défaut $\left(S_{*}^{D_{x}}(1.34)\right)$ est défini par :

$$
S_{*}^{D_{x}}=\operatorname{span}\left\{\left(\begin{array}{l}
1 \\
0 \\
0 \\
0
\end{array}\right),\left(\begin{array}{c}
-1 \\
1 \\
0 \\
0
\end{array}\right),\left(\begin{array}{c}
1 \\
-2 \\
1 \\
0
\end{array}\right)\right\}
$$

Suite à cette définition, le changement de coordonnées (1.39) satisfait :

$$
\frac{\partial \Phi(x(t))}{\partial x(t)}=\left(\begin{array}{cccc}
1 & 0 & 0 & 0 \\
-1 & 1 & 0 & 0 \\
1 & -2 & 1 & 0 \\
0 & 0 & 0 & 1
\end{array}\right)
$$


Dans cette nouvelle base le modèle du système (1.52) s'écrit :

$$
\Sigma_{\widetilde{L}}:\left\{\begin{array}{l}
\dot{\tilde{x}}_{1}(t)=-\tilde{x}_{1}(t)+u_{1}(t)+d(t) \\
\dot{\tilde{x}}_{2}(t)=\tilde{x}_{1}(t)-\tilde{x}_{2}(t)-u_{1}(t) \\
\dot{\tilde{x}}_{3}(t)=-\tilde{x}_{1}(t)+\tilde{x}_{2}(t)-\tilde{x}_{3}(t)+\tilde{x}_{4}(t)+u_{1}(t)+u_{2}(t) \\
\dot{\tilde{x}}_{4}(t)=-\tilde{x}_{1}(t)-2 \tilde{x}_{2}(t)-\tilde{x}_{3}(t) \\
y_{x 1}(t)=\tilde{x}_{1}(t)+2 \tilde{x}_{2}(t)+\tilde{x}_{3}(t) \\
y_{x 2}(t)=\tilde{x}_{4}(t)
\end{array}\right.
$$

A l'aide d'une injection de sortie $\tilde{\Psi}\left(\tilde{x}_{4}(t), u(t), y_{x}(t)\right)=\left(\begin{array}{llll}0 & 0 & 0 & -y_{x 1}(t)\end{array}\right)^{T} \quad$ (cf. (1.42)), nous déterminons le filtre suivant :

$$
\Sigma_{\widetilde{F L}}:\left\{\begin{array}{l}
\dot{\tilde{z}}_{1}(t)=-\tilde{z}_{1}(t)+u_{1}(t) \\
\dot{\tilde{z}}_{2}(t)=\tilde{z}_{1}(t)-\tilde{z}_{2}(t)-u_{1}(t) \\
\dot{\tilde{z}}_{3}(t)=-\tilde{z}_{1}(t)+\tilde{z}_{2}(t)-\tilde{z}_{3}(t)+\tilde{z}_{4}(t)+u_{1}(t)+u_{2}(t) \\
\dot{\tilde{z}}_{4}(t)=-y_{z 1}(t) \\
y_{z 1}(t)=\tilde{z}_{1}(t)+2 \tilde{z}_{2}(t)+\tilde{z}_{3}(t) \\
y_{z 2}(t)=\tilde{z}_{4}(t)
\end{array}\right.
$$

Ainsi, seul l'état estimé par $\tilde{z}_{4}(t)$ est insensible à la perturbation.

Alors la dimension du sous-espace d'état insensible à la perturbation est égale à 1 et la dimension du sous-espace d'état sensible est égale à 3 .

Nous ajoutons que dans ce cas les méthodes (analytique et géométrique) mènent à des résultats équivalents mais ce n'est généralement pas le cas.

Or, à l'aide d'une approche intuitive, nous proposons un filtre possédant des propriétés qui semblent plus intéressantes.

$\underline{\text { Proposition intuitive : }}$

Remarquons que :

$$
x_{1}(t)=y_{1}^{(2)}(t)+2 y_{1}^{(1)}(t)+2 y_{1}(t)-y_{2}(t)-u_{2}(t)-u_{2}^{(1)}(t)
$$


alors nous proposons le filtre associé au modèle du système (1.42) suivant :

$$
\Sigma_{F L}:\left\{\begin{array}{l}
\dot{z}_{1}(t)=-z_{1}(t)+u_{1}(t) \\
\dot{z}_{2}(t)=y_{1}^{(2)}(t)+2 y_{1}^{(1)}(t)+2 y_{1}(t)-y_{2}(t)-u_{2}(t)-u_{2}^{(1)}(t)-z_{2}(t) \\
\dot{z}_{3}(t)=z_{2}(t)-z_{3}(t)+z_{4}(t)+u_{2}(t) \\
\dot{z}_{4}(t)=-z_{3}(t) \\
y_{z 1}(t)=z_{3}(t) \\
y_{z 2}(t)=z_{4}(t)
\end{array}\right.
$$

où $y_{1}^{(2)}(t)+2 y_{1}^{(1)}(t)+2 y_{1}(t)-y_{2}(t)-u_{2}(t)-u_{2}^{(1)}(t)$ est l'injection de sortie.

Ce filtre a l'avantage d'assurer une estimation exacte des états $x_{2}(t), x_{3}(t)$ et $x_{4}(t)$ par $z_{2}(t)$, $z_{3}(t)$ et $z_{4}(t)$.

Alors la dimension du sous-espace d'état insensible à la perturbation est égale à 3 et la dimension du sous-espace d'état sensible est égale à 1.

Notons que l'injection de sortie emploie tant l'ensemble des composantes du vecteur de sortie que les dérivées des sorties. Ainsi elle correspond ni à la forme d'une injection de sortie "analytique" (1.40) ni à la forme d'une injection de sortie "géométrique" (1.42).

Dans ce manuscrit nous nous proposons de formaliser cette approche intuitive à l'aide d'une méthode géométrique systématique visant la détermination explicite de ce type d'injection de sortie mixte.

En effet, nous développons une méthode dans laquelle l'injection de sortie est une fonction non linéaire de tous les signaux connus et de leurs dérivées. L'intérêt principal de cette méthode est d'imposer des conditions de construction moins sévères.

Ensuite, nous détaillons de nouvelles techniques d'analyse structurelle pour la génération de résidus. L'intérêt immédiat est d'accroître le champ d'application des méthodes de détection et de localisation de défauts.

Pour finaliser ces recherches, une détermination explicite de l'injection de sortie est donnée dans le cadre du découplage en respectant les contraintes de convergence exponentielle des estimations imposées a priori.

Cependant, ces travaux nécessitent un certain nombre d'hypothèses. Dans le paragraphe suivant nous en dressons une liste exhaustive.

\subsubsection{Hypothèses de travail}

L'une des premières hypothèses de travail consiste à définir les modèles non linéaires auxquels s'appliquent les méthodes proposées. Dans les premières références ([2]) ([3]) (paragraphe 1.2.2.3) les modèles considérés sont non linéaires généraux (1.3), en revanche, dans 
la paragraphe 1.2.2.4 ils sont affines en la commande (1.5).

Ceci s'explique par leurs sources bibliographiques. Puisque H. Hammouri, A. Isidori et C. De Persis initiateurs d'une méthode géométrique pour l'étude des modèles non linéaires s'intéressaient dans au premier temps à leur commande, les modèles considérés sont non linéaires affines en la commande. En effet, la commande des systèmes non linéaires généraux (1.3) reste difficile et peu employée alors que des méthodes systématiques menant à la commande des modèles affines en la commande existent. Se fondant sur ces travaux, nous étudierons les modèles non linéaires affines en la commande (1.5).

Dans le cadre de travaux théoriques, nous supposerons les modèles "parfaits", c'est-à-dire que les incertitudes de modèle sont inexistantes.

De plus, nous considérons les signaux déterministes et les entrées du système indépendantes, nous parlerons alors d'entrées exogènes.

Dans les chapitres suivants, nous proposons d'autres méthodes de découplage des systèmes. Nous cherchons la plus grande partie de l'état pouvant être rendue insensible à une perturbation.

Ces méthodes permettent ensuite une analyse structurelle pour l'isolation des défauts. Dans ce cadre, il est courant de ne plus distinguer perturbations et défauts car nous chercherons à découpler des défauts. Ainsi seule l'écriture des défauts est considérée.

Pour terminer, nous ajoutons que toutes les études, comparaisons, intersections et dimensions d'espaces sont validées $\forall(x(t), t)$. De la même manière, nous considérons les matrices non-singulières. Ceci n'est pas précisé à chaque fois, afin de ne pas alourdir l'exposé.

En remarque, nous ajoutons que ces hypothèses sont courantes dans l'étude des systèmes non linéaires et n'enlèvent rien aux difficultés des problèmes traités. 


\section{Chapitre 2}

\section{Découplage : méthode géométrique}

En s'inspirant des travaux de C. De Persis et A. Isidori, nous traitons, dans ce chapitre, le découplage des systèmes non linéaires. Sous les hypothèses définies dans la section 1.4.2, nous considérons que l'effet d'une perturbation est découplé s'il existe une partie de l'état du système dont l'estimation $z(t)$ reste exacte malgré l'apparition de la perturbation. La partie de l'état ainsi définie doit être la plus grande possible en terme de dimension car elle définit les capacités de la méthode de découplage. En effet, lors du découplage d'une perturbation, l'objectif est d'estimer avec exactitude la plus grande partie de l'état. Par commodité de calcul, nous recherchons par la suite le plus petit sous-espace d'état sensible à la perturbation via une injection de sortie. Sa détermination est le principal objectif de la phase de découplage.

Dans le cadre du diagnostic, au terme découplage, nous associons également le fait de dissocier les effets des défauts entre eux. Nous contraignons ainsi l'effet d'une partie du défaut à seulement une partie du sous-espace d'état. Si ces sous-espaces, obtenus pour chaque partie du défaut, possèdent des propriétés différentes (en terme de géométrie par exemple) alors la distinction des défauts est possible. A cette fin, une analyse structurelle, s'appuyant sur des inclusions et intersections de sous-espaces d'état minimaux, est proposée. Enfin, des conditions nécessaires et suffisantes de localisation des défauts sont données.

\subsection{Problématique}

Dans cette section, nous rappelons la problématique du découplage des systèmes représentés par un modèle non linéaire (2.1) à l'aide d'un filtre (écriture (2.2)).

$$
\Sigma_{N L}:\left\{\begin{array}{l}
\dot{x}(t)=f_{0}(x(t))+\sum_{i=1}^{m} f_{i}(x(t)) u_{i}(t)+D_{x}(x(t)) d(t) \\
y_{x}(t)=h(x(t))
\end{array}\right.
$$


avec $x(t) \in \mathbb{R}^{n}, u(t) \in \mathbb{R}^{m}, d(t) \in \mathbb{R}^{s}$ et $y_{x}(t) \in \mathbb{R}^{p}$.

$$
\Sigma_{F N L}:\left\{\begin{array}{l}
\dot{z}(t)=f_{0}\left(z(t)+\sum_{i=1}^{m} f_{i}(z(t)) u_{i}(t)+\Psi\left(\bullet_{x}(t), \bullet_{z}(t), \bullet_{u}(t)\right)\right. \\
y_{z}(t)=h(z(t))
\end{array}\right.
$$

où $z(t)$ est une estimation de l'état réel du système $x(t)$ et $\Psi\left(\bullet_{x}(t), \bullet_{z}(t), \bullet_{u}(t)\right)$ est l'injection de sortie. Les variables la composant sont représentées par $\bullet_{x}(t), \bullet_{z}(t)$ et $\bullet_{u}(t)$ qui sont liées respectivement à l'état du système, l'équation du filtre et aux commandes.

Les variables sont soit mesurées (sorties $y_{x}(t)$ ), soit calculables (dérivées des sorties), soit encore connues (commandes).

Le choix de l'injection de sortie fait l'objet de toute notre attention dans ce chapitre car elle conditionne les performances du filtre à savoir la dimension du sous-espace d'état sensible à la perturbation qui doit être minimisée. Ce critère de performance est complété, dans le prochain chapitre, par l'étude de la convergence des estimations du filtre vers les états réels du système.

Nous rappelons l'intérêt de l'injection de sortie en écrivant le filtre dans un nouveau système de coordonnées (défini par $\tilde{z}(t)=\Phi(z(t))$ ) qui a pour objectif de rendre une partie du sous-espace d'état $\left(\tilde{z}_{2}(t)\right)$ insensible à $d(t)$ :

$$
\Sigma_{\widetilde{F N L}}:\left\{\begin{array}{l}
\dot{\tilde{z}}_{1}(t)=\tilde{f}_{0,1}\left(\tilde{z}_{1}(t), \tilde{z}_{2}(t)\right)+\sum_{i=1}^{m} \tilde{f}_{i, 1}\left(\tilde{z}_{1}(t), \tilde{z}_{2}(t)\right) u_{i}(t)+\tilde{\Psi}_{1}\left(\bullet_{x}(t), \bullet_{z}(t), \bullet_{u}(t)\right) \\
\dot{\tilde{z}}_{2}(t)=\tilde{f}_{0,2}\left(\tilde{z}_{1}(t), \tilde{z}_{2}(t)\right)+\sum_{i=1}^{m} \tilde{f}_{i, 2}\left(\tilde{z}_{1}(t), \tilde{z}_{2}(t)\right) u_{i}(t)+\tilde{\Psi}_{2}\left(\bullet_{x}(t), \bullet_{z}(t), \bullet_{u}(t)\right) \\
y_{z}(t)=\tilde{h}\left(\tilde{z}_{1}(t), \tilde{z}_{2}(t)\right)
\end{array}\right.
$$

sous la contrainte :

$$
\tilde{f}_{0,2}\left(\tilde{z}_{1}(t), \tilde{z}_{2}(t)\right)+\sum_{i=1}^{m} \tilde{f}_{i, 2}\left(\tilde{z}_{1}(t), \tilde{z}_{2}(t)\right) u_{i}(t)+\tilde{\Psi}_{2}\left(\bullet_{x}(t), \bullet_{z}(t), \bullet_{u}(t)\right)=\dot{\tilde{x}}_{2}(t), \quad \forall(d(t), u(t), t)
$$

où $\tilde{x}_{2}(t)$ est une partie de l'état du système dans le nouveau système de coordonnées.

En d'autres termes, nous déterminons dans quel cadre l'injection de sortie $\Psi\left(\bullet \bullet_{x}(t), \bullet_{z}(t), \bullet_{u}(t)\right)$ peut exprimer toute contribution de la perturbation dans l'écriture d'une partie de l'équation différentielle. Ces deux sous-espaces d'état engendrés par $\tilde{z}_{1}(t)$ et $\tilde{z}_{2}(t)$ sont libres et générateurs de l'espace d'état et doivent être déterminés par calcul. Le sous-espace sensible à la perturbation est calculé à l'aide d'une séquence non-décroissante ((1.33) par exemple) et augmente ainsi en dimension, au fur et à mesure de la propagation de $d(t)$ au sein de l'espace d'état. Toute la difficulté de l'étude suivante réside dans la minimisation de cette propagation. Nous précisons que les sous-espaces d'état représentés par $\tilde{z}_{1}(t)$ et $\tilde{z}_{2}(t)$ peuvent être réduits au sous-espace d'état nul $\{0\}$. 


\subsection{Rappel d'une méthode de découplage proposée par C. De Persis}

Se fondant sur les notions de sous-espaces invariants et de géométrie différentielle, nous rappelons la solution proposée par C. De Persis et A. Isidori [13], [14], [16] et [17] pour déterminer le plus petit sous-espace sensible à une perturbation.

\subsubsection{Définition théorique}

Cette méthode considère l'écriture particulière de l'injection suivante :

$$
\begin{aligned}
& \tilde{\Psi}_{1}\left(\bullet_{x}(t), \bullet_{z}(t), \bullet \bullet_{u}(t)\right)=0 \\
& \text { et } \\
& \tilde{\Psi}_{2}\left(\bullet_{x}(t), \bullet_{z}(t), \bullet_{u}(t)\right)=\tilde{\Psi}\left(\tilde{z}_{2}(t), u(t), y_{x}(t)\right)-\tilde{\Psi}\left(\tilde{z}_{2}(t), u(t), y_{z}(t)\right)
\end{aligned}
$$

Dans ce cas, nous rappelons l'écriture de la séquence non-décroissante (définie en (1.33)) :

$$
\left\{\begin{array}{l}
S_{0}^{D_{x}}=\operatorname{span}\left\{D_{x}\right\} \\
S_{i+1}^{D_{x}}=\bar{S}_{i}^{D_{x}}+\sum_{k=0}^{m}\left[f_{k}, \bar{S}_{i}^{D_{x}} \cap \operatorname{ker}\{d h\}\right]
\end{array}\right.
$$

avec les conditions d'arrêt :

$$
\bar{S}_{i}^{D_{x}}=S_{i+1}^{D_{x}} \Rightarrow S_{*}^{D_{x}}=\bar{S}_{i}^{D_{x}}
$$

Deux propriétés importantes résultent de la définition de $S_{*}^{D_{x}}$.

La première est la définition précise du plus petit sous-espace d'état sensible à une perturbation $d(t)$, via l'injection de sortie définie en $(2.5)$.

La deuxième est l'existence de $\tilde{z}_{2}(t)(2.3)$ si, et seulement si, le sous-espace d'état engendré par $\left(S_{*}^{D_{x}}\right)^{\perp}$ n'est pas réduit à l'ensemble nul $\{0\}$. Par construction, $\left(S_{*}^{D_{x}}\right)^{\perp}$ est le plus grand sous-espace d'état insensible à une perturbation.

La section suivante présente à l'aide d'un exemple mathématique la manière de mener à bien le calcul du sous-espace d'état insensible à une perturbation. 


\subsubsection{Exemple académique}

Considérons le modèle non linéaire suivant ([25]) :

$$
\Sigma_{N L}:\left\{\begin{array}{l}
\dot{x}(t)=\left(\begin{array}{c}
x_{1}(t) x_{4}(t) \\
x_{3}(t)\left(1-x_{4}(t)\right) \\
0 \\
0
\end{array}\right)+\left(\begin{array}{cc}
0 & 0 \\
0 & 0 \\
0 & x_{1}(t) \\
1 & 0
\end{array}\right)\left(\begin{array}{l}
u_{1}(t)+d_{1}(t) \\
u_{2}(t)+d_{2}(t)
\end{array}\right) \\
y_{x}(t)=\left(\begin{array}{l}
x_{1}(t) \\
x_{2}(t)
\end{array}\right)
\end{array}\right.
$$

où $x(t) \in \mathbb{R}^{4}, u(t) \in \mathbb{R}^{2}, y_{x}(t) \in \mathbb{R}^{2}$ et $d(t) \in \mathbb{R}^{2}$ sont respectivement les états, entrées, sorties et perturbations.

La propagation de chaque perturbation au sein de l'espace d'état est à présent calculée :

$$
\left(\begin{array}{ll}
D_{x 1} & D_{x 2}
\end{array}\right) d(t)=\left(\begin{array}{cc}
0 & 0 \\
0 & 0 \\
0 & x_{1}(t) \\
1 & 0
\end{array}\right) d(t)
$$

Si seule la perturbation $d_{1}(t)$ est considérée, $S_{*}^{D_{x 1}}$ se définit comme suit :

Premier pas

$$
S_{0}^{D_{x 1}}=\operatorname{span}\left\{D_{x 1}\right\}=\operatorname{span}\left\{\left(\begin{array}{l}
0 \\
0 \\
0 \\
1
\end{array}\right)\right\}
$$

Puisqu'une distribution de dimension 1 est toujours involutive, alors $\bar{S}_{0}^{D_{x 1}}=S_{0}^{D_{x 1}}$.

\section{Deuxième pas}

$$
\bar{S}_{0}^{D_{x 1}} \cap \operatorname{ker}\{d h\}=\bar{S}_{0}^{D_{x 1}} \operatorname{car} \operatorname{ker}\{d h\}=\operatorname{span}\left\{\left(\begin{array}{l}
0 \\
0 \\
1 \\
0
\end{array}\right),\left(\begin{array}{l}
0 \\
0 \\
0 \\
1
\end{array}\right)\right\}
$$




$$
\begin{aligned}
S_{1}^{D_{x 1}} & =\bar{S}_{0}^{D_{x 1}}+\left[\left(\begin{array}{c}
x_{1}(t) x_{4}(t) \\
x_{3}(t)\left(1-x_{4}(t)\right) \\
0 \\
0
\end{array}\right),\left(\begin{array}{l}
0 \\
0 \\
0 \\
1
\end{array}\right)\right]+\left[\left(\begin{array}{l}
0 \\
0 \\
0 \\
1
\end{array}\right),\left(\begin{array}{l}
0 \\
0 \\
0 \\
1
\end{array}\right)\right]+\left[\left(\begin{array}{c}
0 \\
0 \\
x_{1}(t) \\
0
\end{array}\right),\left(\begin{array}{l}
0 \\
0 \\
0 \\
1
\end{array}\right)\right]+\operatorname{span}\left\{\left(\begin{array}{l}
0 \\
0 \\
0 \\
0
\end{array}\right)\right\}+\operatorname{span}\left\{\left(\begin{array}{l}
-x_{1}(t) \\
x_{3}(t) \\
0 \\
0 \\
0 \\
0
\end{array}\right)\right\} \\
= & \bar{S}_{0}^{D_{x 1}}+\operatorname{span}\left\{\left(\begin{array}{l}
0 \\
0 \\
0 \\
1
\end{array}\right),\left(\begin{array}{c}
-x_{1}(t) \\
x_{3}(t) \\
0 \\
0
\end{array}\right)\right\}
\end{aligned}
$$

avec

$$
\left[\left(\begin{array}{l}
0 \\
0 \\
0 \\
1
\end{array}\right),\left(\begin{array}{c}
-x_{1}(t) \\
x_{3}(t) \\
0 \\
0
\end{array}\right)\right]=\operatorname{span}\left\{\left(\begin{array}{l}
0 \\
0 \\
0 \\
0
\end{array}\right)\right\}
$$

donc $\bar{S}_{1}^{D_{x 1}}=S_{1}^{D_{x 1}}$

\section{Troisième pas}

$$
\bar{S}_{1}^{D_{x 1}} \cap \operatorname{ker}\{d h\}=\bar{S}_{0}^{D_{x 1}}
$$

Puisque le calcul est identique au précédent, nous concluons en écrivant:

$$
\begin{aligned}
S_{2}^{D_{x 1}}=S_{1}^{D_{x 1}} \Rightarrow S_{*}^{D_{x 1}} & =S_{2}^{D_{x 1}} \\
& S_{*}^{D_{x 1}}=\operatorname{span}\left\{\left(\begin{array}{l}
0 \\
0 \\
0 \\
1
\end{array}\right),\left(\begin{array}{c}
-x_{1}(t) \\
x_{3}(t) \\
0 \\
0
\end{array}\right)\right\}
\end{aligned}
$$

D'une manière identique, $S_{*}^{D_{x 2}}$ est défini par :

$$
S_{*}^{D_{x 2}}=\operatorname{span}\left\{\left(\begin{array}{c}
0 \\
0 \\
x_{1}(t) \\
0
\end{array}\right),\left(\begin{array}{c}
0 \\
-x_{1}(t)\left(1-x_{4}(t)\right) \\
x_{1}(t) x_{4}(t) \\
0
\end{array}\right)\right\}
$$

L'équation (2.15) (respectivement (2.16)) permet la déduction du sous-espace orthogonal (2.17) (respectivement (2.18)) qui correspond au sous-espace d'état insensible, via une injec- 
tion de sortie, à la perturbation $d_{1}(t)$ (respectivement $\left.d_{2}(t)\right)$.

$$
\begin{gathered}
\left(S_{*}^{D_{x 1}}\right)^{\perp}=\operatorname{span}\left\{\left(\begin{array}{l}
0 \\
0 \\
1 \\
0
\end{array}\right),\left(\begin{array}{c}
x_{3}(t) \\
x_{1}(t) \\
0 \\
0
\end{array}\right)\right\} \\
\left(S_{*}^{D_{x 2}}\right)^{\perp}=\operatorname{span}\left\{\left(\begin{array}{l}
1 \\
0 \\
0 \\
0
\end{array}\right),\left(\begin{array}{l}
0 \\
0 \\
0 \\
1
\end{array}\right)\right\}
\end{gathered}
$$

En raison de la non nullité du sous-espace (2.17) (respectivement (2.18)), il existe un filtre permettant la synthèse de l'état $\tilde{z}_{2}(t)$ au sens de (2.3). Ainsi la combinaison d'états $\tilde{x}_{2}(t)$ avec $\left(\tilde{x}_{1}^{T}(t) \tilde{x}_{2}^{T}(t)\right)^{T}=\left(\Phi_{1}^{T}(x(t)) \Phi_{2}^{T}(x(t))\right)^{T}$ peut être estimée par $\tilde{z}_{2}(t)$ sans erreur si $d_{2}(t)=0$, $\operatorname{span}\left\{d \Phi_{1}^{T}\right\}=S_{*}^{D_{x 1}}$ et $\operatorname{span}\left\{d \Phi_{2}^{T}\right\}=\left(S_{*}^{D_{x 1}}\right)^{\perp}$. De même, l'état $\tilde{x}_{2}(t)$ peut être estimé sans erreur par $\tilde{z}_{2}(t)$ si $d_{1}(t)=0, \operatorname{span}\left\{d \Phi_{1}\right\}=S_{*}^{D_{x 2}}$ et $\operatorname{span}\left\{d \Phi_{2}\right\}=\left(S_{*}^{D_{x 2}}\right)^{\perp}$.

La condition d'existence d'un filtre avec les propriétés de sensibilité définies en (2.3) est suffisante mais non nécessaire au découplage des perturbations. En effet, l'injection de sortie a une structure imposée et la séquence non-décroissante (2.6) est initialisée par $\operatorname{span}\left\{D_{x}\right\}$.

Dans la suite de ce chapitre, nous proposons une procédure plus générale de calcul du plus petit sous-espace sensible à une perturbation via une injection de sortie. Les deux restrictions précédemment décrites sont levées afin de déterminer les conditions nécessaires et suffisantes d'existence d'un filtre découplant les perturbations.

\subsection{Proposition d'une méthode de découplage}

Dans le premier chapitre de ce document, deux méthodes assurant le découplage d'un sousespace d'état à l'apparition d'une perturbation ont été rappelées. La dimension de ce sousespace d'état a été étudiée dans le paragraphe 1.2.2.5 en fonction de la forme de l'injection de sortie. Dans cette section nous proposons une méthode géométrique visant à augmenter la dimension du sous-espace d'état insensible à la perturbation par une modification de la forme de l'injection de sortie. La dimension de ce sous-espace est soit précisément déterminée soit estimée à l'aide d'un intervalle borné.

Dans un premier temps, nous étudions la borne supérieure de l'inégalité (définie en (1.43)) :

$$
0 \leq \operatorname{dim}\left(\left(S_{*}^{D_{x}}\right)^{\perp}\right)=\operatorname{dim}(\text { sous-espace d'état insensible à la perturbation } d(t)) \leq n-1
$$


afin d'augmenter la dimension du sous-espace insensible à la perturbation et de montrer que dans certains cas, il est possible de l'étendre à l'espace d'état dans sa totalité. Ensuite, une étude semblable est menée pour la borne inférieure.

Dans une première section, nous nous limitons au découplage mono-perturbation afin d'en faciliter la compréhension. L'étude est généralisée au cas multi-perturbations par la suite. En guise d'introduction, une nouvelle séquence non-décroissante est définie dans l'objectif de calculer le sous-espace maximal atteint par la perturbation sans contrainte de découplage, c'est-à-dire sans l'utilisation d'une injection de sortie.

\subsubsection{Préliminaires}

Afin de mieux cerner le comportement naturel du système, il est intéressant de connaître le sous-espace d'état entaché de l'effet d'une ou plusieurs perturbations. Ceci permet la mise en relief des parties de l'état du système naturellement découplées lorsqu'elles existent. De plus, la définition de ces sous-espaces est d'une grande utilité dans la suite de nos développements et particulièrement lors de l'analyse structurelle pour le diagnostic (section 2.4). A cette fin, nous introduisons une nouvelle séquence non-décroissante :

$$
\left\{\begin{array}{l}
C_{0}^{D_{x}}=\operatorname{span}\left\{D_{x}\right\} \\
C_{i+1}^{D_{x}}=\bar{C}_{i}^{D_{x}}+\sum_{k=1}^{m}\left[f_{k}, \bar{C}_{i}^{D_{x}}\right]
\end{array}\right.
$$

qui se distingue de la séquence (2.6) par l'absence de l'élément ker $\{d h\}$. Cet élément est en effet directement lié à la forme de l'injection de sortie du filtre qui est ici inexistante. Les conditions d'arrêt sont les suivantes:

$$
\bar{C}_{i}^{D_{x}}=C_{i+1}^{D_{x}} \Rightarrow C_{*}^{D_{x}}=\bar{C}_{i}^{D_{x}}
$$

A la distribution $C_{*}^{D_{x}}$ génératrice d'un sous-espace d'état, nous associons le sous-espace entaché de l'effet de la perturbation $d(t)$. Sa distribution orthogonale $\left(C_{*}^{D_{x}}\right)^{\perp}$ est donc indépendante de la perturbation. Suite à un changement de coordonnées $\left(\tilde{x}_{N}(t)=\Phi(x(t))\right)$, nous pouvons récrire le système (2.1) comme suit :

$$
\Sigma_{\widetilde{N L}}:\left\{\begin{array}{l}
\dot{\tilde{x}}_{N 1}(t)=\tilde{f}_{0,1}\left(\tilde{x}_{N 1}(t), \tilde{x}_{N 2}(t)\right)+\sum_{i=1}^{m} \tilde{f}_{i, 1}\left(\tilde{x}_{N 1}(t), \tilde{x}_{N 2}(t)\right) u_{i}(t)+\tilde{D}_{x}\left(\tilde{x}_{N 1}(t), \tilde{x}_{N 2}(t)\right) d(t) \\
\dot{\tilde{x}}_{N 2}(t)=\tilde{f}_{0,2}\left(\tilde{x}_{N 2}(t)\right)+\sum_{i=1}^{m} \tilde{f}_{i, 2}\left(\tilde{x}_{N 2}(t)\right) u_{i}(t) \\
y_{x}(t)=\tilde{h}\left(\tilde{x}_{N}(t)\right)
\end{array}\right.
$$

Dans ce cas, $\tilde{x}_{N 2}(t)$ est l'état du système naturellement découplé. Dans la majorité des cas, ce sous-espace se réduit à l'ensemble nul $\left(\left(C_{*}^{D_{x}}\right)^{\perp}=\{0\}\right)$. 
L'une des premières applications de cette séquence est la détermination des conditions de détectabilité d'un défaut qui jouent un rôle, non seulement lors de l'établissement des conditions de détection et localisation des défauts (section 2.4.2), mais aussi lors de leur découplage.

Un défaut est dit détectable si, et seulement si, l'effet de ce dernier influence les sorties du système. En nous appuyant sur la définition du sous-espace observable $\left(\gamma_{o b s}\right.$, annexe B définition B.1) d'un système non linéaire (2.1), nous traduisons mathématiquement la condition de détectabilité de la manière suivante :

$$
\gamma_{o b s} \nsubseteq\left(C_{*}^{P}\right)^{\perp}
$$

où $P(x(t))$ est la matrice de pondération des défauts.

Bien qu'indépendantes des conditions de découplage du défaut, les conditions de détectabilité sont prises en considération dans l'approche que nous proposons. Dans un premier temps, nous étudions le cas d'une perturbation unique.

\subsubsection{Cas d'une perturbation unique}

Nous considérons ici que la perturbation $d(t)$ est scalaire, et $\operatorname{donc} \operatorname{dim}\left(\operatorname{span}\left\{D_{x}\right\}\right)=1, \forall x(t)$. Nous rappelons que l'injection de sortie que nous considérons lors de cette étude s'écrit $\Psi\left(\bullet_{x}(t), \bullet_{z}(t), \bullet_{u}(t)\right)$ et que les variables utilisées seront définies au fil des pages. Cependant, précisons qu'aucune restriction n'est fixée a priori tant dans les variables utilisées que dans leur ordre de dérivation. L'étude du cas mono-perturbation s'effectue en deux étapes qui sont d'une part le cas où la perturbation est estimable et, d'autre part, le cas où la perturbation n'est pas estimable.

\subsubsection{Perturbation estimable}

Si nous étudions le calcul du sous-espace d'état insensible à une perturbation proposé par C. De Persis (décrit dans la section 2.2), nous remarquons que l'obtention de la borne supérieure $n-1$ (2.19) provient de l'initialisation de la séquence (2.6). En effet, poser $S_{0}^{D_{x}}=\operatorname{span}\left\{D_{x}\right\}$ suppose une impossibilité à rendre insensible à la perturbation le sous-espace d'état représenté par $\operatorname{span}\left\{D_{x}\right\}$.

Ainsi, l'idée principale de notre proposition repose sur l'exploitation des possibilités qu'offrent certains systèmes à exprimer la perturbation à partir des sorties et de leurs dérivées. Nous ajoutons que la recherche d'une injection de sortie assurant l'obtention d'un sous-espace insensible à la perturbation égal à l'espace d'état dans sa totalité équivaut à exprimer la perturbation avec l'injection de sortie. 
Par conséquent, nous déterminons, dans un premier temps les conditions nécessaires et suffisantes à l'expression de la perturbation via une injection de sortie, suivies des conditions suffisantes plus faciles à énoncer en terme de formalisme.

Cependant, nous sommes conscients des contraintes d'applicabilité que représente la détérioration importante des signaux due à de trop nombreuses dérivations. Nous omettrons ces contraintes lors des traitements théoriques, tout en proposant une solution à ce problème (section 2.3.4).

Nous cherchons, dans cette section, à exprimer la perturbation scalaire $d(t)$ à l'aide de l'injection de sortie. Nous supposons $\gamma_{o b s} \nsubseteq\left(C_{*}^{D_{x}}\right)^{\perp}$, ce qui constitue une condition nécessaire à la poursuite de l'étude. En effet, dans le cas contraire, la perturbation ne peut pas être estimée à partir d'une injection de sortie, puisqu'elle n'a pas d'effet sur les sorties.

Sous cette hypothèse, il existe au moins un indice caractéristique modifié $\rho_{m i}^{d}<\infty$ associé à la sortie $y_{i}(t)$ tel que :

$$
\exists i \in\{1, \cdots, p\} / \rho_{m i}^{d}<\infty \Leftrightarrow \gamma_{o b s} \nsubseteq\left(C_{*}^{D_{x}}\right)^{\perp}
$$

A présent, nous définissons de nouvelles notions dans le cadre des systèmes non linéaires [41] comme une extension de celles associées aux systèmes linéaires [46, 23] :

Définition 2.1 Un signal $d(t)$ est dit П-reconstructible si, et seulement si, il existe une fonction $\zeta$ non linéaire en $\Pi$ telle que $d(t)=\zeta(\Pi)$.

De même,

Définition 2.2 Une distribution $\tau(x(t))$ est dite П-reconstructible si, et seulement si, il existe une fonction $\zeta$ non linéaire en $\Pi$ telle que $\tau(x(t))=\frac{\partial}{\partial x(t)} \zeta(\Pi)$.

Ces notions généralisent l'observabilité dans le cadre de l'étude des systèmes linéaires. En effet, si nous étudions l'ensemble des signaux qui définissent le vecteur d'état $x(t)$, et que chaque état est $\Delta$-reconstructible où $\Delta$ est l'ensemble des signaux disponibles (connus) du système, alors le système est observable.

Lors de l'étude des systèmes linéaires, satisfaire les conditions de la définition 2.1 implique la validation des conditions de la définition 2.2 pour la distribution associée au signal. C'est pourquoi étudier l'observabilité de systèmes linéaires équivaut à étudier le rang de matrices car chaque distribution représentée par une ligne de ces matrices est intégrable. Or ce n'est pas le cas lors de l'étude des systèmes non linéaires, toutes les distributions ne sont pas nécessairement intégrables. Cette remarque suscite une nouvelle définition.

Définition 2.3 Un signal $d(t)$ est dit estimable si, et seulement si, il existe une fonction $\zeta$ non linéaire en $\Delta$ telle que $d(t)=\zeta(\Delta)$ où $\Delta$ rassemble les signaux disponibles (connus) $d u$ système. 
Sans perte de généralité, dans la suite de ce manuscrit $\Delta$ représente les signaux suivants :

$$
\Delta=\left[Y_{x}(t), u(t), \cdots, u^{(\max -1)}(t), \xi_{1}\left(Y_{x}(t), Y_{x 1}(t)\right), \cdots, \xi_{l}\left(Y_{x}(t), Y_{x l}(t)\right), \tilde{x}_{N 2}(t)\right]
$$

avec $Y_{x}(t)=\left[y_{x 1}^{(0)}(t), \cdots, y_{x 1}^{\left(\rho_{m 1}^{d}-1\right)}(t), \cdots, y_{x p}^{(0)}(t), \cdots, y_{x p}^{\left(\rho_{m p}^{d}-1\right)}(t)\right]$ et $Y_{x 1}(t), \cdots, Y_{x l}(t)$ des signaux constitués de dérivations des sorties d'indices supérieurs aux $\rho_{m i}^{d}-1$. Les fonctions $\xi_{1}(\bullet), \cdots, \xi_{l}(\bullet)$ ne font pas intervenir dans leur écriture la perturbation ou ses dérivations et max rappelle l'indice de dérivation maximal des sorties de l'ensemble des signaux de $\Delta$.

A titre d'exemple, nous définissons une partie du sous-espace d'état comme suit :

Définition 2.4 Soit $\gamma_{y}$ le sous-espace d'état y-reconstructible, ainsi pour toutes les distributions $\tau(x(t))$ de $\gamma_{y}$ il est possible de trouver une fonction $\Theta$ telle que $\tau(x(t))=\frac{\partial}{\partial x(t)} \Theta(y(t))$

Ces définitions jouent un rôle primordial dans l'établissement des conditions nécessaires et suffisantes d'estimabilité de la perturbation.

Dans un premier temps nous étudions le cas général du signal $\Delta(2.25)$.

\section{Cas général}

Comme nous le précisions précédemment, nous focalisons notre étude sur la recherche des conditions nécessaires et suffisantes d'estimabilité de la perturbation puisqu'elles permettent de vérifier si la dimension du sous-espace d'état sensible à la perturbation $d(t)$ peut être réduite à $\{0\}$. Sous l'hypothèse de synthèse d'une injection de sortie du type $\Psi\left(\bullet_{x}(t), \bullet_{z}(t), \bullet_{u}(t)\right)$, le théorème suivant détermine explicitement les conditions nécessaires et suffisantes d'estimabilité d'une perturbation.

Théorème 2.1 Une perturbation $d(t)$ est estimable si, et seulement si, $d(t)$ est $\Delta$-reconstructible.

Nous précisons que $\Delta$ représente tous les signaux disponibles (connus) du système tout en étant indépendants, dans l'écriture, de $d(t)$.

\section{Preuve}

$\Rightarrow$ Si $d(t)$ est estimable, alors il existe une fonction non linéaire de signaux permettant l'écriture de $d(t)$. Or, les signaux utilisés dans cette expression font obligatoirement partis de l'ensemble $\Delta$ puisqu'aucune restriction n'est effectuée dans la composition de cet ensemble.

$\Leftarrow$ Ce sens d'implication est évident puisque si d(t) est $\Delta$-reconstructible, il existe par définition une fonction non linéaire $\zeta$ telle que $\zeta(\Delta)=d(t)$. Ainsi, la perturbation $(d(t))$ est estimable. 
Dans le cadre du filtrage, il est donc possible de déterminer une injection de sortie répondant aux égalités suivantes si la perturbation est estimable :

$$
\Psi\left(\bullet_{x}(t), \bullet_{z}(t), \bullet_{u}(t)\right)=\Psi(\Delta)=d(t)
$$

Ceci permet l'écriture suivante du filtre :

$$
\Sigma_{F N L}:\left\{\begin{array}{l}
\dot{z}(t)=f_{0}(z(t))+\sum_{i=1}^{m} f_{i}(z(t)) u_{i}(t)+D_{x}(z(t)) \Psi(\Delta) \\
y_{z}(t)=h(z(t))
\end{array}\right.
$$

L'estimation de l'état $z(t)$ est alors insensible à l'apparition de la perturbation $d(t)$. En d'autres termes, nous obtenons dans ce cas :

$$
\operatorname{dim}(\text { sous-espace d'état insensible à la perturbation } d(t))=n
$$

Cette égalité assure l'augmentation de la dimension du sous-espace d'état insensible à la perturbation $d(t)$ puisqu'elle était précédemment limitée à $n-1$ (2.19).

Dans certains cas, il paraît plus facile d'estimer l'effet de la perturbation $D_{x}(x(t)) d(t)$. Cette affirmation se justifie principalement par le fait que la pondération de la perturbation par $D_{x}(x(t))$ est une fonction de l'état. En considérant l'exemple où $D_{x}(x(t))$ est une fonction d'état non $\Delta$-reconstructible, la difficulté apparaît.

Théorème 2.2 L'effet d'une perturbation $d(t)$ est estimable si, et seulement si, $D_{x}(x(t)) d(t)$ est $\Delta$-reconstructible.

Nous précisons que $\Delta$ représente tous les signaux disponibles (connus) du système tout en étant indépendants, dans l'écriture, de $d(t)$.

\section{Preuve}

La preuve de ce théorème est identique à la précédente avec l'expression de $D_{x}(x(t)) d(t)$ comme étant l'effet d'une perturbation.

Si nous posons :

$$
\Psi\left(\bullet_{x}(t), \bullet_{z}(t), \bullet_{u}(t)\right)=\Psi(\Delta)=D_{x}(x(t)) d(t)
$$

alors il est possible d'écrire le filtre de la manière suivante :

$$
\Sigma_{F N L}:\left\{\begin{array}{l}
\dot{z}(t)=f_{0}(z(t))+\sum_{i=1}^{m} f_{i}(z(t)) u_{i}(t)+\Psi(\Delta) \\
y_{z}(t)=h(z(t))
\end{array}\right.
$$


De même que lors de l'écriture du filtre (2.27), l'estimation de l'état $z(t)$ est alors insensible à l'apparition de la perturbation $d(t)$ et :

$$
\operatorname{dim}(\text { sous-espace d'état insensible à la perturbation } d(t))=n
$$

Cette égalité assure l'augmentation de la dimension du sous-espace d'état insensible à la perturbation $d(t)$ (définie en $(2.19)$ ).

Si la synthèse de l'un de ces filtres est possible ((2.27) et (2.30)), alors l'estimation de l'état du système $x(t)$ par $z(t)$ est exacte dans sa totalité $\forall(d(t), t)$. La condition de construction du filtre associé à l'écriture (2.30) étant moins restrictive que pour l'écriture (2.27), nous identifions les conditions nécessaires et suffisantes de découplage total (l'espace d'état dans sa totalité est insensible à la perturbation) comme étant les suivantes :

Théorème 2.3 Un système est totalement découplable si, et seulement si, l'effet de la perturbation est $\Delta$-reconstructible.

\section{Preuve}

$\Rightarrow$ Si $D_{x}(x(t)) d(t)$ est $\Delta$-reconstructible, alors il existe une fonction non linéaire de signaux connus égale à $D_{x}(x(t)) d(t)$. Ainsi, il est possible d'écrire le système sous la forme (2.30). $L$ 'ensemble de l'état estimé par $z(t)$ est alors exact $\forall d(t)$ et le système est dit totalement découplable.

$\Leftarrow$ Si le système est totalement découplable alors il existe un filtre estimant exactement l'état dans sa totalité $\forall d(t)$. Puisque l'écriture de l'équation différentielle fait intervenir $d(t)$, ceci nécessite donc

$l a$

$\Delta$-reconstructibilité de l'effet de la perturbation.

Le théorème précédent nous permet d'établir les propriétés suivantes.

Proposition 2.1 Les 3 propriétés suivantes sont équivalentes :

(i) $D_{x}(x(t)) d(t)$ est $\Delta$-reconstructible;

(ii) le sous-espace d'état sensible à la perturbation $d(t)$ se réduit à $\{0\}$;

(iii) la dimension du sous-espace d'état insensible à la perturbation d(t) est égale à $n$.

\section{Preuve}

(i) $\Rightarrow\left(\right.$ ii) Si $D_{x}(x(t)) d(t)$ est $\Delta$-reconstructible, alors le système est totalement découplable (d'après le théorème 2.3). Un système totalement découplable étant un système dont la totalité de l'état peut être rendue insensible à la perturbation, il en résulte que le sousespace d'état sensible à la perturbation se réduit à $\{0\}$. 
(ii) $\Rightarrow$ (iii) Cette implication est triviale. En effet, puisque l'espace d'état est de dimension n, l'espace d'état insensible à la perturbation (sous-espace d'état orthogonal au sous-espace sensible à la perturbation) est nécessairement de dimension $n$.

(iii) $\Rightarrow$ (i) Si la dimension du sous-espace d'état insensible est égale à $n$ alors le système est dit totalement découplable et, d'après le théorème 2.3, cette propriété implique la $\Delta$ reconstructibilité de l'effet de la perturbation $D_{x}(x(t)) d(t)$.

Comparé à l'inégalité (2.19) le point (iii) de la proposition 2.1 reflète bien l'augmentation de la dimension du sous-espace d'état insensible à la perturbation $d(t)$ grâce à la synthèse appropriée de l'injection de sortie générale $\Psi\left(\bullet_{x}(t), \bullet_{z}(t), \bullet_{u}(t)\right)=\Psi(\Delta)$.

La notion de sous-espace $\Delta$-reconstructible étant relativement abstraite, nous nous proposons d'étudier un cas particulier donnant lieu à une définition plus concrète de $\Delta$. Cependant, en raison des hypothèses qui doivent être satisfaites, seules des conditions suffisantes au problème du découplage des perturbations sur un système non linéaire peuvent être déduites. L'intérêt de l'étude d'un tel cas particulier réside dans la mise en évidence des différentes étapes nécessaires à l'estimation de la perturbation ou de son effet à l'aide de signaux explicites et possédant un sens physique.

\section{Cas particulier}

En première hypothèse notée $H 1$, nous supposons que l'ensemble des signaux connus est défini par :

$$
\Delta_{t}=\left[y_{x 1}^{(0)}(t), \cdots, y_{x 1}^{\left(\rho_{m 1}^{d}-1\right)}(t), \cdots, y_{x p}^{(0)}(t), \cdots, y_{x p}^{\left(\rho_{m p}^{d}-1\right)}(t), u(t), \cdots, u^{\left(\max _{i}\left(\rho_{m i}^{d}\right)-1\right)}(t), \tilde{x}_{N 2}(t)\right]
$$

où $\tilde{x}_{N 2}(t)$ représente le sous-espace d'état naturellement découplé de la perturbation (en relation avec $\left(C_{*}^{D_{x}}\right)^{\perp}$ et l'écriture du système $\left.(2.22)\right)$.

$\Delta_{t}$ est ainsi un sous-ensemble de $\Delta(2.25)$ car tous les signaux $\xi_{i}(\bullet), i=\{1, \cdots, l\}$ sont omis. Cette définition assure le fait que la perturbation $d(t)$ n'intervient dans aucune écriture en raison de la définition même des indices caractéristiques employés $\left(\rho_{m i}^{d}\right)$.

Afin de faciliter l'étude de la perturbation $d(t)$ et plus précisément son effet sur les sorties, nous définissons un vecteur de sensibilité comme suit :

Définition 2.5 Nous appelons vecteur de sensibilité des sorties à la perturbation $d(t)$, le vecteur $V^{D_{x}}$ défini par:

$$
V^{D_{x}}=\left(\begin{array}{c}
V_{1}^{D_{x}} \\
\vdots \\
V_{p}^{D_{x}}
\end{array}\right)=\left(\begin{array}{c}
y_{x 1}^{\left(\rho_{m 1}^{d}\right)}(t) \\
\vdots \\
y_{x p}^{\left(\rho_{m p}^{d}\right)}(t)
\end{array}\right)
$$


suite à la convention liée à la définition 1.5 si $\rho_{m i}^{d} \rightarrow \infty$ alors $y_{x p}^{\left(\rho_{m i}^{d}\right)}(t)=0$.

Sous l'hypothèse de détectabilité notée H2, il existe toujours au moins une composante $(i \in\{1, \cdots, p\})$ du vecteur de sensibilité des sorties à la perturbation telle que :

$V_{i}^{D_{x}}(x(t), U(t), d(t))=y_{x i}^{\left(\rho_{m i}^{d}\right)}(x(t), U(t), d(t))=\bar{f}_{0, i}(x(t))+\bar{f}_{1, i}(x(t), U(t))+\bar{D}_{x, i}(x(t), U(t)) d(t)$

avec $U=\left[u^{(0)}(t), \cdots, u^{\left(\rho_{m i}^{d}-1\right)}(t)\right], \bar{f}_{0, i}, \bar{f}_{1, i}$ et $\bar{D}_{x, i}$ des fonctions non linéaires obtenues par dérivation de la $i^{\mathrm{e}}$ sortie. Nous insistons sur la structure particulière du système que nous considérons (système non linéaire affine en la commande) qui assure une équation affine en $d(t)$.

Par la suite, nous considérons que le vecteur de sensibilité est la source de toutes les expressions de la perturbation ou de son effet. Cette hypothèse est en fait une troisième restriction car l'écriture de combinaisons de sorties $\left(y_{x i}(t)\right)$ et de leurs dérivées (d'ordre $k i \geq \rho_{m i}^{d}$ en l'occurrence) où seul $d(t)$ interviendrait (sans les dérivées successives de $d(t)$ ) est possible pour une géométrie particulière :

$\lambda\left(y_{x 1}(t), \cdots, y_{x 1}^{(k 1)}(t), \cdots, y_{x p}(t), \cdots, y_{x p}^{(k p)}(t)\right)=\lambda_{0}(x(t))+\lambda_{1}(x(t), U(t))+\lambda_{2}(x(t), U(t)) d(t)$

où $\lambda, \lambda_{0}, \lambda_{1}$ et $\lambda_{2}$ sont des fonctions non linéaires analytiques.

Sous les hypothèses H1 et H2 respectivement associées aux équations (2.32) et (2.34), les conditions suffisantes à l'estimation de la perturbation ou de son effet sont déterminées à l'aide du théorème 2.4 .

Théorème 2.4 Si le couple $\quad\left(V_{i}^{D_{x}}(x(t), U(t), d(t)=0), \frac{\partial V_{i}^{D_{x}}(x(t), U(t), d(t))}{\partial d(t)}\right) \quad$ est $\Delta_{t}$-reconstructible alors la perturbation est estimable.

\section{Preuve}

Ce théorème énonce une condition liée à l'estimation de la perturbation d(t). Cette condition nécessitant la satisfaction des deux hypothèses H1 et H2, il ne peut s'agir que d'une condition suffisante.

$\Rightarrow$ Si le couple $\left(V_{i}^{D_{x}}(x(t), U(t), d(t)=0), \frac{\partial V_{i}^{D_{x}}(x(t), U(t), d(t))}{\partial d(t)}\right)$ est $\Delta_{t}$-reconstructible alors il existe deux fonctions $\zeta_{1}$ et $\zeta_{2}$ telles que $V_{i}^{D_{x}}(x(t), U(t), d(t))=\zeta_{1}\left(\Delta_{t}\right)+\zeta_{2}\left(\Delta_{t}\right) d(t)$ avec $V_{i^{D_{x}}}^{D_{x}}(x(t), U(t), d(t))=y_{i}^{\left(\rho_{m i}^{d}\right)}(t)$. Ainsi il est possible d'écrire sous ces conditions $: d(t)=$ $\frac{V_{i}^{D_{x}}(x(t), U(t), d(t))-\zeta_{1}\left(\Delta_{t}\right)}{\zeta_{2}\left(\Delta_{t}\right)}$. Nous insistons sur le fait que nous étudions un signal scalaire justifiant ainsi l'inversibilité de $\zeta_{2}\left(\Delta_{t}\right)$.

Dans ces mêmes termes, il est possible d'estimer l'effet d'une perturbation. 
Théorème 2.5 Si l'effet de la perturbation $D_{x}(x(t)) d(t)$ est $\Delta_{t}$-reconstructible alors l'effet de la perturbation est estimable.

\section{Preuve}

La preuve est identique à celle proposée dans le théorème 2.2. Comme précédemment, il ne peut s'agir que d'une condition suffisante de part la restriction des signaux $\Delta \grave{a} \Delta_{t}$.

Sous l'hypothèse d'estimation de la perturbation ou de son effet, nous savons que le sousespace d'état sensible à la perturbation se réduit à l'ensemble $\{0\}$. Ainsi, en utilisant les signaux connus $\Delta_{t}(2.32)$ il est possible de réaliser un découplage total de la perturbation, i.e. :

$$
\begin{gathered}
\Psi\left(\Delta_{t}\right) \\
\Downarrow
\end{gathered}
$$

$\operatorname{dim}($ sous-espace d'état insensible à la perturbation $d(t))=n$

En conclusion de l'étude réalisée, nous rappelons que l'augmentation de la dimension du sous-espace insensible à la perturbation visant un découplage total n'est possible que si la perturbation $d(t)$ ou son effet $D_{x}(x(t)) d(t)$ est estimable. Les conditions nécessaires et suffisantes à l'estimation de la perturbation $d(t)$ par l'utilisation d'une injection de sortie générale $(\Psi(\Delta))$ sont énoncées de même que les conditions suffisantes si un nombre réduit de signaux est considéré dans la définition de cette même injection de sortie $\Psi\left(\Delta_{t}\right)\left(\Delta_{t}\right.$ est une troncature de $\Delta$ ).

Si l'une des hypothèses d'utilisation des théorèmes 2.1, 2.2, 2.4 et 2.5 est satisfaite alors la dimension du sous-espace d'état insensible à la perturbation $d(t)$ est augmentée de $n-1$ (2.19) à $n$ (2.35). Ce résultat constitue directement une amélioration à la méthode proposée par C. De Persis (section 2.2).

Cependant la perturbation (ou son effet) n'est pas toujours estimable, nous montrons à l'aide de l'étude suivante qu'il est tout de même possible d'augmenter la dimension du sous-espace d'état insensible à la perturbation.

\subsubsection{Perturbation non estimable}

L'étude qui suit n'est réalisée que dans le cas où la perturbation n'est pas estimable (au sens des théorèmes 2.1 et 2.2 ) et dans ce cas nous avons :

$$
\operatorname{dim}(\text { sous-espace d'état insensible à la perturbation } d(t))<n
$$

Au même titre que C. De Persis, nous proposons une séquence pour le calcul du plus petit sous-espace sensible à une perturbation via une injection de sortie. Puisque l'injection de sortie que nous considérons dans ce paragraphe est générale du point de vue des signaux qu'elle 
emploie, nous augmentons nécessairement la dimension du sous-espace d'état insensible à une perturbation (2.19).

\section{Séquence}

Pour déterminer le plus petit sous-espace d'état sensible à $d(t)$, nous étudions la propagation de la perturbation à travers l'espace d'état. Considérant que nous avons déjà calculé $\bar{S}_{m, k}^{D_{x}}$, il est alors possible d'écrire le système (2.1) comme suit :

$$
\Sigma_{\widetilde{N L}}:\left\{\begin{array}{l}
\dot{\tilde{x}}_{1}^{k}(t)=\tilde{f}_{0,1}^{k}\left(\tilde{x}_{1}^{k}(t), \tilde{x}_{2}^{k}(t)\right)+\sum_{i=1}^{m} \tilde{f}_{i, 1}^{k}\left(\tilde{x}_{1}^{k}(t), \tilde{x}_{2}^{k}(t)\right) u_{i}(t)+\tilde{D}_{x}^{k}\left(\tilde{x}_{1}^{k}(t), \tilde{x}_{2}^{k}(t)\right) d(t) \\
\dot{\tilde{x}}_{2}^{k}(t)=\tilde{f}_{0,2}^{k}\left(\tilde{x}_{1}^{k}(t), \tilde{x}_{2}^{k}(t)\right)+\sum_{i=1}^{m} \tilde{f}_{i, 2}^{k}\left(\tilde{x}_{1}^{k}(t), \tilde{x}_{2}^{k}(t)\right) u_{i}(t) \\
y_{x}(t)=\tilde{h}\left(\tilde{x}_{1}^{k}(t), \tilde{x}_{2}^{k}(t)\right)
\end{array}\right.
$$

à partir d'un changement de coordonnées $\tilde{x}^{k}(t)=\Phi_{k}(x(t))$, où $k$ représente l'indice d'itération de la séquence et $\frac{\partial \Phi_{k}(x(t))}{\partial x(t)}=\left(\bar{S}_{m, k}^{D_{x}}\left(\bar{S}_{m, k}^{D_{x}}\right)^{\perp}\right)^{T}$.

L'objectif de cette écriture, est d'étudier s'il est possible de transcrire, à l'aide d'une injection de sortie, l'effet de la perturbation dans l'expression de $\dot{\tilde{x}}_{2}^{k}(t)$. A cette fin, nous cherchons à exprimer toutes les composantes de $\tilde{x}_{1}^{k}(t)$ intervenant dans l'écriture de $\dot{\tilde{x}}_{2}^{k}(t)$ grâce à une fonction non linéaire des signaux connus.

Lors de la définition des signaux connus $\Delta$ (cf. équation (2.25)) et $\Delta_{t}$ (cf. équation (2.32)), $\tilde{x}_{N 2}(t)$ est considéré comme insensible à la perturbation $d(t)$ et est donc parfaitement connu. En supposant qu'il est possible de découpler le système tel qu'il est écrit équation (2.37) alors $\tilde{x}_{2}^{k}(t)$ devient insensible à la perturbation et fait alors partie des signaux connus reflétés $\operatorname{par} \Delta^{k}$ :

$$
\Delta^{k}=\left[Y, U_{\max 1}(t), \xi_{1}\left(Y(t), Y_{1}(t)\right), \cdots, \xi_{l}\left(Y(t), Y_{l}(t)\right), \tilde{x}_{2}^{k}(t)\right]
$$

avec $Y(t)=\left[y_{1}^{(0)}(t), \cdots, y_{1}^{\left(\rho_{m 1}^{d}-1\right)}(t), \cdots, y_{p}^{(0)}(t), \cdots, y_{p}^{\left(\rho_{m p}^{d}-1\right)}(t)\right]$

$U_{\max 1}(t)=\left[u(t), \cdots, u^{(\max -1)}(t)\right], Y_{1}(t), \cdots, Y_{l}(t)$ des signaux constitués de dérivations des sorties d'indices supérieurs aux $\rho_{m i}^{d}-1$. Les fonctions $\xi_{1}(\bullet), \cdots, \xi_{l}(\bullet)$ ne font pas intervenir dans leur écriture la perturbation ou ses dérivations et max rappelle l'indice de dérivation maximal des sorties de l'ensemble des signaux de $\Delta^{k}$.

Notons que pour $k=0, \tilde{x}_{2}^{k}(t)=\tilde{x}_{N 2}(t)$.

Ainsi, nous scindons $\bar{S}_{m, k}^{D_{x}}$ en deux sous-espaces générateurs avec :

- $S_{m \overline{\Delta^{k}}}^{D_{x}}$ : la partie du sous-espace $\bar{S}_{m, k}^{D_{x}}$ non $\Delta^{k}$-reconstructible intervenant dans l'écriture de $\dot{\tilde{x}}_{2}^{k}(t)$,

- $S_{m \Delta^{k}}^{D_{x}}$ : la partie du sous-espace $S_{m \Delta^{k}}^{D_{x}} \subseteq \operatorname{span}\left\{\left(d x_{1}^{k}\right)^{T}\right\}$ incluant la partie $\Delta^{k}$-reconstructible, 
tels que :

$$
\bar{S}_{m, k}^{D_{x}}=S_{m \Delta^{k}}^{D_{x}} \oplus S_{m \overline{\Delta^{k}}}^{D_{x}}
$$

Si le sous-espace d'état non $\Delta^{k}$-reconstructible $\left(S_{m \frac{x}{\Delta^{k}}}^{D_{x}}\right)$ est différent de $\{0\}$, nous savons que la perturbation va continuer à se propager au sein de l'espace d'état. Et d'autres parties de l'espace d'état non engendrées par span $\left\{\left(d x_{1}^{k}\right)^{T}\right\}$ seront sensibles à la perturbation en raison de l'impossibilité à exprimer toutes les composantes de $\tilde{x}_{1}^{k}(t)$ intervenant dans l'écriture de $\dot{\tilde{x}}_{2}^{k}(t)$ à l'aide de l'injection de sortie. Ainsi nous précisons que $\operatorname{span}\left\{\left(d x_{2}^{k}\right)^{T}\right\} \subseteq \operatorname{span}\left\{\left(d x_{2}^{k+1}\right)^{T}\right\}, \forall k$.

Nous traduisons la propagation de la perturbation à l'aide d'une séquence non-décroissante :

$$
S_{m, k+1}^{D_{x}}=\bar{S}_{m, k}^{D_{x}}+\sum_{i=0}^{m}\left[\tilde{f}_{i}^{k}\left(\tilde{x}_{1}^{k}(t), \tilde{x}_{2}^{k}(t)\right), S_{m \bar{\Delta}^{k}}^{D_{x}}\right]
$$

du fait des inclusions successives : $\operatorname{span}\left\{D_{x}\right\} \subseteq \bar{S}_{m, 1}^{D_{x}} \subseteq \cdots \subseteq \bar{S}_{m, k}^{D_{x}} \subseteq \cdots \subseteq S_{m *}^{D_{x}}$ où $S_{m *}^{D_{x}}$ est le sous-espace d'état recherché, cette propagation est obligatoirement limitée par $\operatorname{dim}\left(S_{m *}^{D_{x}}\right) \leq$ $\operatorname{dim}\left(\operatorname{span}\left\{d x^{T}\right\}\right)$ puisque nous étudions des systèmes de dimension finie $\left(\operatorname{dim}\left(\operatorname{span}\left\{d x^{T}\right\}\right)=\right.$ $n)$.

Ainsi, il existe $k_{1} \in \mathbb{N}$ tel que :

$$
\bar{S}_{m, k_{1}}^{D_{x}}=S_{m, k_{1}+1}^{D_{x}} \Rightarrow S_{m *}^{D_{x}}=\bar{S}_{m, k_{1}}^{D_{x}}
$$

Avec cette égalité (2.41), nous assurons l'existence d'une injection de sortie telle que :

$$
\dot{\tilde{x}}_{2}^{k}(t)=\breve{f}_{0,2}^{k}\left(\tilde{x}_{2}^{k}(t)\right)+\sum_{i=1}^{m} \breve{f}_{i, 2}^{k}\left(\tilde{x}_{2}^{k}(t)\right) u_{i}(t)+\tilde{\Psi}_{1}^{k}\left(\Delta_{x}\right)+\sum_{i=1}^{m} \tilde{\Psi}_{i}^{k}\left(\Delta_{x}\right) u_{i}(t)
$$

avec $\breve{f}_{i \bullet}^{1}=\tilde{f}_{i \bullet}^{1}+\tilde{\Psi}_{i}^{1}\left(\Delta_{z}^{1}\right)$ où $i=\{0, \cdots, m\}$.

En rappelant le cas déjà évoqué lors de l'écriture de l'inégalité (2.19), nous n'avions pas pu déterminer, d'une manière générale, la borne inférieure. En revanche, nous avions précisé que cette dernière était fortement liée au sous-espace d'état $\operatorname{span}\left\{d h^{T}\right\}$ ou plus particulièrement à ker $\{d h\}$. Chaque modèle possédant une géométrie qui lui est propre, nous ne pouvons pas généraliser la valeur prise par la borne inférieure malgré l'utilisation d'une injection de sortie plus générale que précédemment. Cependant l'intérêt de l'étude que nous menons dans cette section est mis en relief, en précisant que la borne inférieure ne peut être qu'améliorée ou maintenue. En effet, l'injection de sortie de l'équation (2.19) n'étant qu'un cas particulier de $\Psi(\Delta)$, la borne inférieure que nous proposons est au moins égale à celle obtenue à l'aide des travaux de C. De Persis et A. Isidori. Nous précisons que la borne inférieure est fortement liée au sous-espace d'état engendré par $\operatorname{span}\left\{\left(d \Delta^{k}\right)^{T}\right\}$ et insistons sur le fait que $\operatorname{span}\left\{d h^{T}\right\} \subseteq \operatorname{span}\left\{\left(d \Delta^{k}\right)^{T}\right\}, \forall k$. 
Afin de finaliser l'étude, nous supposons que $\left(S_{m *}^{D_{x}}\right)^{\perp}=\left(\bar{S}_{m, k_{1}}^{D_{x}}\right)^{\perp} \neq\{0\}$, et qu'il existe ainsi un sous-espace d'état insensible à la perturbation $d(t)$. Sous cette hypothèse, il est possible d'écrire le système (2.37) comme suit :

$$
\Sigma_{\widetilde{N L}}:\left\{\begin{array}{l}
\dot{\tilde{x}}_{1}^{k_{1}}(t)=\tilde{f}_{0,1}^{k_{1}}\left(\tilde{x}_{1}^{k_{1}}(t), \tilde{x}_{2}^{k_{1}}(t)\right)+\sum_{i=1}^{m} \tilde{f}_{i, 1}^{k_{1}}\left(\tilde{x}_{1}^{k_{1}}(t), \tilde{x}_{2}^{k_{1}}(t)\right) u_{i}(t)+\tilde{D}_{x}^{k_{1}}\left(\tilde{x}_{1}^{k_{1}}(t), \tilde{x}_{2}^{k_{1}}(t)\right) d(t) \\
\dot{\tilde{x}}_{2}^{k_{1}}(t)=\tilde{f}_{0,2}^{k_{1}}\left(\tilde{x}_{1}^{k_{1}}(t), \tilde{x}_{2}^{k_{1}}(t)\right)+\sum_{i=1}^{m} \tilde{f}_{i, 2}^{k_{1}}\left(\tilde{x}_{1}^{k_{1}}(t), \tilde{x}_{2}^{k_{1}}(t)\right) u_{i}(t) \\
y_{x}(t)=\tilde{h}\left(\tilde{x}_{1}^{k_{1}}(t), \tilde{x}_{2}^{k_{1}}(t)\right)
\end{array}\right.
$$

où la transformation $\tilde{x}_{*}^{k_{1}}(t)=\left(\left(\tilde{x}_{1}^{k_{1}}(t)\right)^{T}\left(\tilde{x}_{2}^{k_{1}}(t)\right)^{T}\right)^{T}=\Phi_{k_{1}}(x(t))$ est obtenue à l'aide du difféomorphisme défini par :

$$
\frac{\partial \Phi_{k_{1}}(x(t))}{\partial x(t)}=\left(\begin{array}{c}
\left(\bar{S}_{m, k_{1}}^{D_{x}}\right)^{T} \\
\left(\left(\bar{S}_{m, k_{1}}^{D_{x}}\right)^{\perp}\right)^{T}
\end{array}\right)
$$

En conclusion, nous pouvons ajouter que le sous-espace d'état associé à $\tilde{x}_{2}^{k 1}(t)$ est découplé de la perturbation. De plus, il est bon de rappeler qu'il s'agit du plus grand sous-espace d'état insensible à la perturbation quelle que soit l'injection de sortie employée.

\subsubsection{Conclusion}

Dans cette section, nous avons cherché à augmenter la dimension du sous-espace d'état insensible à la perturbation via une injection de sortie. Cette injection de sortie peut être qualifiée de généralisée car l'ensemble des signaux qui la constitue sont sans restriction et tout à fait généraux. L'équation (2.35) et ce qui précède permettent, à l'aide de l'injection de sortie $\Psi(\Delta)$, d'encadrer la dimension du sous-espace d'état insensible à la pertubation comme suit :

$0 \leq \operatorname{dim}\left(S_{*}^{D_{x}}\right) \leq \operatorname{dim}\left(S_{m *}^{D_{x}}\right)=\operatorname{dim}($ sous-espace d'état insensible à la perturbation $d(t)) \leq n$

où les sous-espaces d'état $S_{*}^{D_{x}}$ et $S_{m *}^{D_{x}}$ sont respectivement définis par les séquences (2.40) et (1.33).

En comparant avec l'inégalité (2.19) nous notons une augmentation des deux bornes atteignables. Cependant seul le cas mono-perturbation a été considéré, et c'est pourquoi nous proposons d'étendre notre étude au cas de perturbations multiples.

\subsubsection{Cas de perturbations multiples}

Dans cette section, nous supposons $\operatorname{dim}(d(t))=s$ et $\operatorname{dim}\left(\operatorname{span}\left\{D_{x}(x(t))\right\}\right)=s, \forall x(t)$ et notre objectif est de découpler l'ensemble du vecteur perturbation. Comme dans la section 
précédente, nous proposons une étude de découplage en deux étapes.

La première étape est de déterminer précisément la dimension du sous-espace insensible au vecteur perturbation si celui-ci est estimable.

Elle précède une deuxième étape visant l'étude de la propagation de la perturbation à travers l'espace d'état et conduit à la diminution de la dimension du sous-espace d'état sensible aux perturbations.

\subsubsection{Perturbations estimables}

Deux nouvelles notations sont introduites : $d_{j}(t)$ est la $j^{\mathrm{e}}$ composante du vecteur des perturbations $d(t)\left(\operatorname{dim}\left(d_{j}(t)=1\right)\right.$ et $\overline{d_{j}}(t)$ est constitué de l'ensemble des perturbations privées de $d_{j}(t)$ soit $\overline{d_{j}}(t)=\left(\begin{array}{llllll}d_{1}(t) & \cdots & d_{j-1}(t) & d_{j+1}(t) & \cdots & d_{s}(t)\end{array}\right)^{T}$. Nous précisons l'écriture des pondérations associées aux signaux précédents :

$$
D_{x}(x(t)) d(t)=\left(\begin{array}{lll}
D_{x 1}(x(t)) & \cdots & D_{x s}(x(t))
\end{array}\right)\left(\begin{array}{c}
d_{1}(t) \\
\vdots \\
d_{s}(t)
\end{array}\right)=\sum_{j=1}^{s} D_{x j}(x(t)) d_{j}(t)
$$

En liaison avec le déroulement de la section consacrée à l'étude du cas mono-perturbation, nous commençons par énoncer les conditions nécessaires et suffisantes basées sur une écriture générale de l'injection de sortie. Puis une écriture plus restrictive mais moins abstraite de l'injection de sortie mène à l'établissement de conditions suffisantes à l'estimation de l'ensemble des perturbations. Dans ces deux cas, nous montrons que la dimension du sous-espace insensible aux perturbations est supérieure à celle donnée par l'inégalité (2.19).

\section{Cas général}

Le cas multi-perturbations ne pose pas de problèmes particulier dans le sens où les théorèmes liés à l'estimation d'une perturbation (théorème 2.1) ou de son effet (théorème 2.2) s'appliquent encore. Cependant, il faut préciser la définition de l'ensemble des signaux disponibles (connus) qui diffère de la définition (2.25). En effet, lorsque nous désirons estimer une perturbation $\left(d_{j}(t)\right)$ ou son effet, nous souhaitons que cette estimation soit parfaitement indépendante des autres perturbations $\left(\overline{d_{j}}(t)\right)$. Nous proposons d'écrire l'ensemble des signaux connus $\Delta$ comme suit :

$$
\Delta=\left[Y_{x}(t), u(t), \cdots, u^{(\max -1)}(t), \xi_{1}\left(Y_{x}(t), Y_{x 1}(t)\right), \cdots, \xi_{l}\left(Y_{x}(t), Y_{x l}(t)\right), \tilde{x}_{N 2}(t)\right]
$$

avec $Y_{x}(t)=\left[y_{x 1}^{(0)}(t), \cdots, y_{x 1}^{\left(\min _{j}\left(\rho_{m 1}^{d_{j}}\right)-1\right)}(t), \cdots, y_{x p}^{(0)}(t), \cdots, y_{x p}^{\left(\min _{j}\left(\rho_{m p}^{d_{j}}\right)-1\right)}(t)\right], Y_{x 1}(t), \cdots, Y_{x l}(t)$ des signaux constitués de dérivations des sorties d'indices supérieurs aux $\left(\min _{j}\left(\rho_{m i}^{d_{j}}\right)-1\right)$. Les fonctions $\xi_{1}(\bullet), \cdots, \xi_{l}(\bullet)$ ne font pas intervenir dans leur écriture les perturbations ou leurs dérivations. max rappelle l'indice de dérivation maximal des sorties de l'ensemble des 
signaux de $\Delta$.

L'étude du cas multi-perturbations, bien que similaire en de nombreux points au cas monoperturbation, se justifie par la différence importante au niveau de l'ensemble des signaux sélectionnés au sein de $\Delta$ d'une part et la nécessité d'insister sur "le traitement simultané" du vecteur $d(t)$ d'autre part. En effet, nous proposons, non pas l'étude des composantes $d_{j}(t)$ une à une mais plutôt, le traitement du vecteur perturbation dans sa totalité. Ceci explique le choix de l'indice de dérivation $\left(\min _{j}\left(\rho_{m i}^{d_{j}}\right)-1\right)$ car toute dérivation $y_{i}^{\left(\min _{j}\left(\rho_{m i}^{d_{j}}\right)-1+k\right)}(t), \forall k>0$ ferait nécessairement intervenir une composante de la perturbation et/ou une de ses dérivations.

Ainsi la différence avec le cas mono-perturbation apparaît au travers des indices de dérivation de chaque sortie dans l'expression de $\Delta$. Et ceci ne peut être que le résultat d'un "traitement simultané".

En nous appuyant sur la définition de $\Delta(c f .(2.47))$ et la définition 2.25, nous étendons les deux théorèmes (2.1 et 2.2) au cas multi-perturbations.

Théorème 2.6 Le vecteur perturbation $d(t)$ est estimable dans sa totalité si, et seulement si, le vecteur $d(t)$ est $\Delta$-reconstructible.

Nous précisons que $\Delta$ représente tous les signaux disponibles (connus) du système tout en étant indépendants, dans l'écriture, de toute composante de $d(t)$.

Théorème 2.7 L'effet du vecteur perturbation $d(t)$ est estimable dans sa totalité si, et seulement si, $D_{x}(x(t)) d(t)$ est $\Delta$-reconstructible.

Nous précisons que $\Delta$ représente tous les signaux disponibles (connus) du système tout en étant indépendants, dans l'écriture, de toute composante $d(t)$.

En référence à la proposition 2.1, nous établissons la proposition suivante :

Proposition 2.2 Les 3 propriétés suivantes sont équivalentes :

(i) $D_{x}(x(t)) d(t)$ est $\Delta$-reconstructible

(ii) $S_{m *}^{D_{x}}=\{0\}$

(iii) $\operatorname{dim}\left(\operatorname{span}\left\{\left(S_{m_{*}^{*}}^{D_{x}}\right)^{\perp}\right\}\right)=n$

Les preuves de cette proposition et des théorèmes 2.6 et 2.7 sont semblables en tous points à celles énoncées dans le cas mono-perturbation (section 2.3.2).

Satisfaire l'un des théorèmes 2.6 ou 2.7 assure le découplage dit total du système.

Précisons que la présence d'états dans la signature des perturbations peut poser problème dans certains cas.

Dans le paragraphe suivant, nous étudions un cas particulier de troncature de $\Delta$. 


\section{Cas particulier}

En relation avec le vecteur de sensibilité des sorties à une perturbation (2.33), nous définissons la matrice de sensibilité $M^{D_{x}}$.

Définition 2.6 Nous appelons matrice de sensibilité des sorties aux perturbations $d_{j}(t)$ avec $j=\{1, \cdots, s\}$, la matrice $M^{D_{x}}$ définie par :

$$
M^{D_{x}}=\left(\begin{array}{ccc}
y_{x 1}^{\left(\rho_{m 1}^{d_{1}}\right)}(t) & \cdots & y_{x 1}^{\left(\rho_{m 1}^{d_{s}}\right)}(t) \\
\vdots & \ddots & \vdots \\
y_{x p}^{\left(\rho_{m p}^{d_{1}}\right)}(t) & \cdots & y_{x p}^{\left(\rho_{m p}^{d_{s}}\right)}(t)
\end{array}\right)=\left(\begin{array}{lll}
V^{D_{x 1}} & \cdots & V^{D_{x s}}
\end{array}\right)
$$

suite à la convention liée à la définition 1.5 si $\rho_{m i}^{d_{j}} \rightarrow \infty$ alors $y_{x p}^{\left(\rho_{m i}\right)}(t)=0$.

Une définition particulière mais plus concrète de $\Delta$ est donnée par :

$$
\begin{array}{r}
\Delta_{t}=\left[y_{x 1}^{(0)}(t), \cdots, y_{x 1}^{\left(\min _{j}\left(\rho_{m 1}^{d_{j}}\right)-1\right)}(t), \cdots, y_{x p}^{(0)}(t), \cdots, y_{x p}^{\left(\min _{j}\left(\rho_{m p}^{d_{j}}\right)-1\right)}(t)\right. \\
\left.u(t), \cdots, u^{\left(\max _{i}\left(\min _{j} \rho_{m i}^{d_{j}}\right)-1\right)}(t), \tilde{x}_{N 2}(t)\right]
\end{array}
$$

Remarquons la prise en considération de l'ensemble des perturbations dans le choix des signaux connus au travers les indices de dérivation des sorties.

De manière à simplifier l'étude, nous définissons, à partir de l'écriture de l'équation (2.48), la matrice $M_{s}^{D_{x}}=\left\{M_{s(i, j)}^{D_{x}}\right\}$ comme suit : $M_{s(i, j)}^{D_{x}}=1$ si les deux conditions $(i)$ et $(i i)$ ci-dessous sont vérifiées, sinon $M_{s(i, j)}^{D_{x}}=0$.

(i) le couple $\left(M_{(i, j)}^{D_{x}}\left(x(t), U, \bar{d}_{j}(t), d_{j}(t)=0\right), \frac{\partial M_{(i, j)}^{D_{x}}\left(x(t), U, \bar{d}_{j}(t), d_{j}(t)\right)}{\partial d_{j}(t)}\right)$ est $\Delta_{t}$-reconstructible,

(ii) $\rho_{i}^{d_{j}}=\min _{j}\left(\rho_{i}^{d_{j}}\right)$.

La première condition $(i)$, s'appuyant sur la forme particulière des systèmes non linéaires étudiés (affines en la commande), généralise celle énoncée dans le théorème 2.4. La deuxième condition $(i i)$ reflète les possibles interactions entre plusieurs perturbations sur une même sortie.

La matrice binaire $M_{s}^{D_{x}}$ (constituée de "0" et de "1") permet de conclure sur les conditions d'estimabilité de l'ensemble du vecteur perturbation en se référant au théorème suivant.

Théorème 2.8 Si la matrice $M_{s}^{D_{x}}$ est de rang $s \forall(x(t), u(t))$ alors l'ensemble du vecteur perturbation $d(t)$ est estimable.

\section{Preuve}

Ce théorème énonce une condition liée à l'estimabilité du vecteur perturbation $d(t)$. Elle 
repose sur les deux hypothèses effectuées auparavant (restriction de $\Delta \grave{a} \Delta_{t}$ et seuls les signaux $y_{i}^{\left(\rho_{m i}^{d_{j}}\right)}(t),(i \in\{1, \cdots, s\})$ sont disponibles à l'estimation de $\left.d_{j}(t)\right)$. En ce sens il ne peut s'agir que d'une condition suffisante.

$\Rightarrow$ Si le couple $\left(M_{(i, j)}^{D_{x}}\left(x(t), U, \bar{d}_{j}(t), d_{j}(t)=0\right), \frac{\partial M_{(i, j)}^{D_{x}}\left(x(t), U, \bar{d}_{j}(t), d_{j}(t)\right)}{\partial d_{j}(t)}\right)$ est $\Delta_{t}$-reconstructible alors il existe deux fonctions $\zeta_{1}$ et $\zeta_{2}$ de $\Delta_{t}$ telles que $M_{(i, j)}^{D_{x}}\left(x(t), U, \bar{d}_{j}(t), d_{j}(t)\right)=\zeta_{1}\left(\Delta_{t}\right)+$ $\zeta_{2}\left(\Delta_{t}\right) d(t)$ avec $M_{(i, j)}^{D_{x}}\left(x(t), U, \bar{d}_{j}(t), d_{j}(t)\right)=y_{i}^{\left(\rho_{m i}^{d_{j}}\right)}(t)$. Cette égalité est conditionnée par la vérification de (i). Cependant, celle-ci n'est pas suffisante à l'estimation du vecteur perturbation. Chacune des composantes $d_{j}(t)$ ne peut être estimée indépendamment des autres que si la condition (ii) est vérifiée. Dans le cas contraire, d'autres perturbations interviennent dans l'écriture de la sortie concernée et il n'est pas possible d'exprimer $d_{j}(t) \grave{a}$ partir des signaux $\Delta_{t}$. Enfin, l'estimation de l'ensemble du vecteur perturbation est assurée que si toutes les composantes $d_{j}(t)$ pour $j=\{1, \cdots, s\}$ sont estimables et dans ce cas le rang de la matrice $M_{s}^{D_{x}}$ est égal à s.

Remarquons que l'hypothèse courante $\operatorname{dim}\left(y_{x}(t)\right) \geq \operatorname{dim}(d(t))$ est nécessaire pour la vérification de la condition de rang.

En référence au théorème 2.5, nous exprimons la $\Delta_{t}$-reconstructibilité de l'effet de la perturbation $D_{x}(x(t)) d(t)$.

Théorème 2.9 Si l'effet de la perturbation $D_{x}(x(t)) d(t)$ est $\Delta_{t}$-reconstructible alors l'effet de la perturbation est estimable.

\section{Preuve}

La preuve est identique à celle proposée pour le théorème 2.5.

Pour résumer, ce paragraphe décrit des conditions suffisantes mais non nécessaires au découplage dit total d'un vecteur perturbations (l'ensemble de l'espace d'état est insensible à la perturbation via une injection de sortie).

En conclusion de cette section, si l'une des hypothèses d'utilisation des théorèmes 2.6, 2.7, 2.8 et 2.9 est satisfaite alors il est possible de réaliser le découplage total du vecteur perturbation, i.e. :

$$
\begin{gathered}
\Psi(\Delta) \text { ou } \Psi\left(\Delta_{t}\right) \text { suivant les théorèmes } \\
\Downarrow
\end{gathered}
$$

$\operatorname{dim}($ sous-espace d'état insensible à la perturbation $d(t))=n$

A l'issue de ces résultats, nous développons un nouvel algorithme visant à la détermination du plus petit sous-espace d'état insensible à une perturbation. 


\subsubsection{Perturbations non estimables}

La considération du cas multi-perturbations ne change pas beaucoup la méthodologie décrite dans la section 2.3.2 (initialisation mise à part). Nous considérons le vecteur perturbation $d(t)$. Si cette dernière n'est pas totalement estimable (condition du théorème 2.7) alors le système n'est pas totalement découplable $\left(S_{m_{*}}^{D_{x}} \neq\{0\}\right)$. Cependant, contrairement au cas mono-perturbation, l'initialisation de la séquence n'est pas égale à $\operatorname{span}\left\{D_{x}\right\}$ car dans le cas multi-perturbations, une partie des perturbations peut être estimable sans que l'autre le soit et nous avons alors :

$\operatorname{dim}($ sous-espace d'état insensible à la perturbation $d(t))$

$$
\leq n-\operatorname{dim}(\text { partie estimable de la pertubation })<n
$$

Ainsi, si $S_{m \overline{\Delta^{0}}}^{D_{x}}$ représente les distributions qui pondérent la partie non estimable des perturbations à l'aide de l'ensemble des signaux $\Delta$ (défini en 2.47 ) noté ici $\Delta^{0}$ et en posant :

$$
\bar{S}_{m, 1}^{D_{x}}=\bar{S}_{m \overline{\Delta^{0}}}^{D_{x}}
$$

la récurrence suivante est définie :

$$
S_{m, k+1}^{D_{x}}=\bar{S}_{m, k}^{D_{x}}+\sum_{i=0}^{m}\left[\tilde{f}_{i}^{k}\left(\tilde{x}_{1}^{k}(t), \tilde{x}_{2}^{k}(t)\right), S_{m \overline{\Delta^{k}}}^{D_{x}}\right]
$$

Rappelons que $S_{m \overline{\Delta^{k}}}^{D_{x}}$ représente le sous-espace d'état non $\Delta^{k}$-reconstructible sensible à la perturbation. L'indexation de $\Delta$ joue un rôle important car il est nécessaire de considérer dans l'expression de $\Delta^{k}$ les signaux $\tilde{x}_{2}^{k}(t)$ qui sont en relation avec l'indice d'itération de la récurrence, ainsi :

$$
\Delta^{k}=\left[Y_{x}(t), u(t), \cdots, u^{(\max -1)}(t), \xi_{1}\left(Y_{x}(t), Y_{x 1}(t)\right), \cdots, \xi_{l}\left(Y_{x}(t), Y_{x l}(t)\right), \tilde{x}_{2}^{k}(t)\right]
$$

avec $Y_{x}(t)=\left[y_{x 1}^{(0)}(t), \cdots, y_{x 1}^{\left(\min _{j}\left(\rho_{m 1}^{d_{j}}\right)-1\right)}(t), \cdots, y_{x p}^{(0)}(t), \cdots, y_{x p}^{\left(\min _{j}\left(\rho_{m p}^{d_{j}}\right)-1\right)}(t)\right], Y_{x 1}(t), \cdots, Y_{x l}(t)$ des signaux constitués de dérivations des sorties d'indices supérieurs aux $\left(\min _{j}\left(\rho_{m i}^{d_{j}}\right)-1\right)$. Les fonctions $\xi_{1}(\bullet), \cdots, \xi_{l}(\bullet)$ ne font pas intervenir dans leur écriture les perturbations ou leurs dérivations. max rappelle l'indice de dérivation maximal des sorties de l'ensemble des signaux de $\Delta^{k}$.

Nous insistons sur la présence de $\tilde{x}_{2}^{k}(t)$ qui est liée au sous-espace d'état $\operatorname{span}\left\{\left(d \tilde{x}_{2}^{k}\right)^{T}\right\}=$ $\left(S_{m, k}^{D_{x}}\right)^{\perp}$.

\subsubsection{Conclusion}

Dans cette section, nous avons cherché à augmenter la dimension du sous-espace d'état $S_{m *}^{D_{x}}$ insensible au vecteur perturbation via une injection de sortie. L'équation (2.50) et le raisonnement qui précède permettent d'encadrer le sous-espace d'état sensible à la perturbation 
comme suit :

$\Psi(\Delta)$
$\Downarrow$
$0 \leq \operatorname{dim}\left(S_{*}^{D_{x}}\right) \leq \operatorname{dim}\left(S_{m *}^{D_{x}}\right)=\operatorname{dim}($ sous-espace d'état insensible à la perturbation $d(t)) \leq n$

où $S_{m *}^{D_{x}}$ est le résultat d'une étude avec l'injection de sortie $\Psi(\Delta)$.

En comparant avec l'inégalité (2.19) nous notons l'amélioration des deux bornes atteignables.

\subsubsection{Contraintes physiques}

Lors de l'étude des cas mono et multi-perturbations, les mesures et leurs dérivées successives sont considérées. Or, nous n'ignorons pas qu'en pratique, le calcul des dérivations temporelles est difficilement réalisable. Dans cette section, nous ne prétendons pas résoudre tous les problèmes qui y sont liés mais nous proposons d'adapter la méthode présentée afin d'en améliorer sa mise en oeuvre.

En raison des différents bruits de mesure, il peut s'avérer qu'à partir d'un certain indice de dérivation $\left(m_{j}\right.$ associé à la sortie $\left.j\right)$, les signaux deviennent inacceptables en terme de qualité.

La qualité d'un signal se juge, dans ce cas, par l'information qu'il porte (au sens large). Cette information, en général basse fréquence, peut se trouver de plus en plus détériorée par de multiples dérivations au profit du bruit par exemple, généralement haute fréquence. En effet, dériver un signal s'apparente à le filtrer à l'aide d'un filtre passe haut.

Ainsi, lors de la recherche du plus petit sous-espace d'état insensible à une perturbation, nous considérons l'écriture de $\Delta^{k}$ comme suit :

$$
\Delta^{k}=\left[y_{x 1}^{(0)}(t), \cdots, y_{x 1}^{\left(m_{1}\right)}(t), \cdots, y_{x p}^{(0)}(t), \cdots, y_{x p}^{\left(m_{p}\right)}(t), u(t), \cdots, u^{(\max -1)}(t), \tilde{x}_{2}^{k}(t)\right]
$$

avec un déroulement de l'algorithme restant identique. Il est évident que lors de cette écriture, nous considérons $m_{j} \leq\left(\min _{j}\left(\rho_{m 1}^{d_{j}}\right)-1\right), \forall j$. Nous ajoutons que la méthode développée par C. De Persis et A. Isidori, est un cas particulier de celle-ci, en considérant $m_{j}=0, \forall j$.

Afin de mettre en relief les avantages des méthodes proposées, plusieurs cas sont étudiés. 


\subsubsection{Exemples académiques}

\subsubsection{Exemple 1}

Nous considérons un système décrit par le modèle suivant :

$$
\left\{\begin{array}{l}
\dot{x}_{1}(t)=x_{2}(t) x_{3}(t)+d(t) \\
\dot{x}_{2}(t)=-x_{1}(t) x_{2}(t)+x_{3}^{2}(t) u(t) \\
\dot{x}_{3}(t)=x_{2}(t)-x_{3}(t) \\
y_{x 1}(t)=x_{2}(t) \\
y_{x 2}(t)=x_{3}(t)
\end{array}\right.
$$

avec $D_{x}(x(t))=\left(\begin{array}{lll}1 & 0 & 0\end{array}\right)^{T}$.

Il est facile de vérifier en dérivant successivement les sorties qu'il existe une expression de la perturbation $d(t)$.

En effet, nous montrons que :

$$
\begin{aligned}
d(t)=-\left(y_{x 1}^{(2)}(t)-2 y_{x 2}(t) y_{x 2}^{(1)}(t) u(t)\right. & \left.-y_{x 2}^{2}(t) u^{(1)}(t)\right) \frac{1}{y_{x 1}(t)} \\
& +\left(y_{x 1}^{(1)}(t)-y_{x 2}^{2}(t) u(t)\right) \frac{y_{x 1}^{(1)}(t)}{y_{x 1}^{2}(t)}-y_{x 1}(t) y_{x 2}(t)
\end{aligned}
$$

En nous référant au théorème 2.3 , nous précisons que le système présenté est totalement découplable puisque le défaut est $\Delta$-reconstructible.

Il en résulte que la dimension du sous-espace d'état découplé est égale à la dimension de l'état.

En revanche, l'expression du signal $d(t)$ (2.58) fait apparaître les dérivées premières et secondes des sorties. Dans un cadre réel, ceci pouvant être une source de problèmes, nous considérons à présent que seuls les signaux non dérivés des sorties et commandes sont utilisables. En calculant la séquence $C_{*}^{D_{x}}(2.20)$ qui traduit le plus grand espace sensible à la perturbation $d(t)$, nous pouvons conclure qu'aucun sous-espace d'état n'est naturellement découplé. Puisque $\operatorname{dim}\left(C_{*}^{D_{x}}\right)=\operatorname{dim}(x(t))$, la distribution $\Delta^{0}$ se réduit à :

$$
\Delta^{0}=\left[y_{x 1}(t), y_{x 2}(t), u(t)\right]
$$

où nous remarquons l'absence de toute dérivée et de l'état naturellement découplé $\tilde{x}_{N 2}(t)$.

La perturbation $d(t)$ étant non $\Delta^{0}$-reconstructible et de dimension 1 , la séquence non décroissante est initialisée par $\operatorname{span}\left\{D_{x}\right\}$, i.e. $\bar{S}_{m \bar{\Delta}^{0}}^{D_{x}}=\operatorname{span}\left\{D_{x}\right\}$ (en référence à l'équation $(2.52))$.

Nous rappelons l'écriture des fonctions et de leurs matrices jacobiennes :

$$
f_{0}(x(t))=\left(\begin{array}{c}
x_{2}(t) x_{3}(t) \\
-x_{1}(t) x_{2}(t) \\
x_{2}(t)-x_{3}(t)
\end{array}\right) \quad f_{1}(x(t))=\left(\begin{array}{c}
0 \\
x_{3}^{2}(t) \\
0
\end{array}\right)
$$




$$
\frac{\partial f_{0}(x(t))}{\partial x(t)}=\left(\begin{array}{ccc}
0 & x_{3}(t) & x_{2}(t) \\
-x_{2}(t) & -x_{1}(t) & 0 \\
0 & 1 & -1
\end{array}\right) \quad \frac{\partial f_{1}(x(t))}{\partial x(t)}=\left(\begin{array}{ccc}
0 & 0 & 0 \\
0 & 0 & 2 x_{3}(t) \\
0 & 0 & 0
\end{array}\right)
$$

Suite à une première itération, nous trouvons $S_{m, 1}^{D_{x}}=\operatorname{span}\left\{\left(\begin{array}{l}1 \\ 0 \\ 0\end{array}\right),\left(\begin{array}{c}0 \\ x_{2}(t) \\ 0\end{array}\right)\right\}$. Il est facile de montrer que $S_{m, 1}^{D_{x}}$ est involutive, donc $\bar{S}_{m, 1}^{D_{x}}=S_{m, 1}^{D_{x}}$. Une nouvelle définition des signaux utilisables est donnée ( $c f$. l'équation (2.54)) :

$$
\Delta^{1}=\left[y_{x 1}(t), y_{x 2}(t), u(t), x_{3}(t)\right]
$$

Afin de séparer le sous-espace d'état sensible à la perturbation du sous-espace pouvant devenir insensible à la perturbation, via une injection de sortie, le système de coordonnées est transformé par difféomorphisme :

$$
\tilde{x}^{1}(t)=\Phi_{1}(x(t))=\left(\begin{array}{ccc}
1 & 0 & 0 \\
0 & 1 & 0 \\
0 & 0 & 1
\end{array}\right)
$$

avec $x_{2}(t) \neq 0$,

$$
\operatorname{span}\left\{\left(\begin{array}{l}
1 \\
0 \\
0
\end{array}\right),\left(\begin{array}{l}
0 \\
1 \\
0
\end{array}\right)\right\}=\bar{S}_{m, 1}^{D_{x}} \quad \text { et } \quad \text { span }\left\{\left(\begin{array}{l}
0 \\
0 \\
1
\end{array}\right)\right\}=\left(\bar{S}_{m, 1}^{D_{x}}\right)^{\perp}
$$

Ainsi le système peut se récrire comme suit :

$$
\left\{\begin{array}{l}
\dot{\tilde{x}}_{1}^{1}(t)=\left(\begin{array}{l}
\dot{x}_{1}(t) \\
\dot{x}_{2}(t)
\end{array}\right)=\left(\begin{array}{c}
x_{2}(t) x_{3}(t)+d(t) \\
-x_{1}(t) x_{2}(t)+x_{3}^{2}(t) u(t)
\end{array}\right) \\
\dot{\tilde{x}}_{2}^{1}(t)=\dot{x}_{3}(t)=x_{2}(t)-x_{3}(t) \\
y_{x 1}(t)=x_{2}(t) \\
y_{x 2}(t)=x_{3}(t)
\end{array}\right.
$$

Nous remarquons l'apparition de l'état $x_{2}(t)$ au sein de l'équation régissant la dynamique de $\tilde{x}_{2}^{1}(t)$. Cependant, ce signal étant $\Delta^{1}$-reconstructible, il est aisé d'assurer $S_{m *}^{D_{x}}=S_{m, 1}^{D_{x}}$ via une injection de sortie.

En conclusion de cet exemple, il apparait qu'un découplage total est possible si les dérivées des sorties sont prises en compte dans l'injection de sortie (le sous-espace d'état insensible à la perturbation $d(t)$ (découplée) est alors de dimension 3). En revanche, en proscrivant 
l'utilisation des dérivées de sortie, la dimension du sous-espace d'état insensible à la perturbation $d(t)$ est, cette fois, réduite à 1 . Il parait alors évident, sur cet exemple, que la troncature des signaux utilisés pour générer l'injection de sortie joue un rôle d'importance dans les performances du découplage.

\subsubsection{Exemple 2}

Dans ce nouvel exemple, nous considérons le modèle du système suivant :

$$
\left\{\begin{array}{l}
\dot{x}_{1}(t)=-x_{1}^{2}(t)+u_{1}(t)+d_{1}(t) \\
\dot{x}_{2}(t)=-x_{2}(t)+x_{3}(t) u_{2}(t) \\
\dot{x}_{3}(t)=x_{2}(t)-x_{3}(t) x_{2}(t)^{2} d_{2}(t)+u_{3}(t) \\
\dot{x}_{4}(t)=-x_{3}(t) x_{4}(t) \\
y_{x 1}(t)=x_{1}(t) \\
y_{x 2}(t)=x_{4}(t)
\end{array}\right.
$$

avec $\left(D_{x 1}(x(t)) \quad D_{x 2}(x(t))\right)=\left(\begin{array}{cc}1 & 0 \\ 0 & 0 \\ 0 & -x_{3}(t) x_{2}^{2}(t) \\ 0 & 0\end{array}\right)$.

Nous pouvons montrer que $d_{1}(t)=y_{x 1}^{(1)}(t)+y_{x 1}^{2}(t)-u_{1}(t)$ donc $S_{m *}^{D_{x 1}}=\{0\}$. En revanche, ni la perturbation $d_{2}(t)$ ni son effet est estimable en raison l'état $x_{2}(t)$. Ainsi, la séquence non décroissante est initialisée par $S_{m, 1}^{D_{x 2}}=\operatorname{span}\left\{\left(\begin{array}{llll}0 & 0 & -x_{3}(t) x_{2}^{2}(t) & 0\end{array}\right)^{T}\right\}$. De même que dans le cas précédent nous définissons le changement de coordonnées avec : $\tilde{x}^{1}(t)=\Phi_{1}(x(t))=$ $\left(\begin{array}{llll}0 & 0 & 1 & 0 \\ 1 & 0 & 0 & 0 \\ 0 & 1 & 0 & 0 \\ 0 & 0 & 0 & 1\end{array}\right)$

avec $x_{3}(t) x_{2}^{2}(t) \neq 0$

$$
\operatorname{span}\left\{\left(\begin{array}{l}
0 \\
0 \\
1 \\
0
\end{array}\right)\right\}=\bar{S}_{m, 1}^{D_{x 2}} \quad \text { et } \operatorname{span}\left\{\left(\begin{array}{l}
1 \\
0 \\
0 \\
0
\end{array}\right),\left(\begin{array}{l}
0 \\
1 \\
0 \\
0
\end{array}\right),\left(\begin{array}{l}
0 \\
0 \\
0 \\
1
\end{array}\right)\right\}=\left(\bar{S}_{m, 1}^{D_{x 2}}\right)^{\perp}
$$


et nous récrivons le système comme suit :

$$
\left\{\begin{array}{l}
\dot{\tilde{x}}_{1}^{1}(t)=\dot{x}_{3}(t)=x_{2}(t)-x_{3}(t) x_{2}(t)^{2} d_{2}(t)+u_{3}(t) \\
\dot{\tilde{x}}_{2}^{1}(t)=\left(\begin{array}{l}
\dot{x}_{1}(t) \\
\dot{x}_{2}(t) \\
\dot{x}_{4}(t)
\end{array}\right)=\left(\begin{array}{c}
-x_{1}^{2}(t)+u_{1}(t)+d_{1}(t) \\
-x_{2}(t)+x_{3}(t) u_{2}(t) \\
-x_{3}(t) x_{4}(t)
\end{array}\right) \\
y_{x 1}(t)=x_{1}(t) \\
y_{x 2}(t)=x_{4}(t)
\end{array}\right.
$$

L'apparition de l'état $x_{3}(t)$ sensible à la perturbation $d_{2}(t)$ au sein des équations régissant $\dot{\tilde{x}}_{2}^{1}(t)$ n'est pas gênante puisque $x_{3}(t)$ est $\Delta^{1}$-reconstructible $\left(x_{3}(t)=\frac{-y_{x 2}^{(1)}(t)}{y_{x 2}(t)}\right)$. De ce fait, le sous-espace d'état sensible à la perturbation peut être restreint via une injection de sortie à $\operatorname{span}\left\{\left(d \tilde{x}_{1}^{1}\right)^{T}\right\}=\operatorname{span}\left\{\left(\begin{array}{llll}0 & 0 & 1 & 0\end{array}\right)^{T}\right\}$. En d'autres termes $S_{m *}^{D_{x 2}}=\bar{S}_{m, 1}^{D_{x 2}}=S_{m, 1}^{D_{x 2}}$.

Cet exemple a permis de mettre en relief les propositions de la section 2.3.3.

Dans un premier temps, nous avons cherché les composantes estimables de la perturbation qui se résument à $d_{1}(t)\left(S_{m *}^{D_{x}}=\{0\}\right)$. Il en résulte que la dimension du sous-espace d'état insensible à la perturbation $d_{1}(t)$ est $n$ si l'injection de sortie est définie en fonction des signaux $\Delta$.

Dans un deuxième temps, puisque la perturbation $d_{2}(t)$ n'est pas estimable, nous avons développé la séquence (2.53) dans le but de maximiser la dimension du sous-espace d'état insensible à $d_{2}(t)$. Il en résulte que cette dimension est égale à $\operatorname{dim}\left\{\left(S_{m, 1}^{D_{x 2}}\right)^{\perp}\right\}=3$, puisque l'état $x_{3}(t)$ est reconstructible en utilisant des dérivées de sortie.

Pour conclure ces commentaires, nous ajoutons que si nous avions utilisé la séquence (1.33), les performances auraient été réduites à deux niveaux. En effet, le sous-espace d'état sensible à la perturbation $d_{1}(t)$ aurait été de dimension 1 (au lieu de 0 ) d'une part et l'espace d'état sensible à la perturbation $d_{2}(t)$ aurait été de dimension 2 (au lieu de 1) d'autre part.

Dans le cadre du diagnostic, rendre insensible une partie de l'état d'un filtre à un défaut permet de distinguer dans certains cas les effets de plusieurs défauts et donc de les isoler. Différents cas de figure sont étudiés dans la section suivante.

\subsection{Analyse structurelle pour le diagnostic}

Dans cette section, une méthode visant l'établissement de conditions nécessaires et suffisantes à la détection et l'isolation de défauts est proposée. Dans un premier temps, nous rappelons les conditions d'existence d'une solution au F.P.R.G. proposées dans la littérature. Puis nous effectuons une analyse structurelle géométrique afin d'affaiblir les conditions d'isolation 
des défauts. Cette analyse consiste en l'étude géométrique du comportement des défauts à travers l'espace d'état permettant la détermination de conditions géométriques d'isolation. Enfin nous illustrons les résultats obtenus à l'aide d'exemples.

\subsubsection{Rappel des objectifs}

Il est important d'établir dans quel cadre s'inscrit notre étude. A l'aide d'une approche géométrique, nous visons l'établissement de nouvelles conditions d'isolabilité des défauts. Par abus de langage, lorsque nous qualifions d'isolables des défauts, cela signifie qu'ils possèdent des comportements différents sur l'espace d'état engendré par les sorties ( $\gamma_{y}$ définition 2.4). Ainsi, nous ne considérons pas la dynamique que peuvent avoir les défauts sur les sorties et leurs dérivées. Or cette dynamique peut parfois être un moyen pour les distinguer.

Cette remarque sera le point de départ de la réflexion proposée dans le chapitre d'ouverture où une définition plus générique que l'isolabilité géométrique est proposée.

Nous débutons notre étude par le rappel des conditions d'isolation des défauts (résolution du F.P.R.G.) établies dans [13, 15] et utilisées dans [14, 16, 17] par C. De Persis et A. Isidori.

Nous précisons que les conditions d'isolation énumérées dans la suite sont obtenues avec une injection de sortie de la forme $\Psi\left(y_{x}(t), u(t), \tilde{x}_{2}(t)\right)$ ne reposant sur aucune dérivée des sorties. Les conditions d'isolation seront donc suffisantes mais non nécessaires.

Nous rappelons qu'il existe une solution au problème fondamental de génération de résidus si à chaque défaut $w_{j}(t)(j=\{1, \cdots, q\})$, il est possible d'associer une fonction $\chi^{\overline{P_{j}}}(\bullet)$ telle que :

$$
Q_{*}^{\overline{P_{j}}} \cap \operatorname{span}\{d h\}=\operatorname{span}\left\{d\left(\chi^{\overline{P_{j}}} \circ h(x(t))\right)\right\}
$$

$$
\left(\operatorname{span}\left\{d\left(\chi^{\overline{P_{j}}} \circ h(x(t))\right)\right\}\right)^{\perp} \cap \operatorname{span}\left\{P_{j}\right\}=\{0\}
$$

avec $\operatorname{span}\left\{\overline{P_{j}}\right\}=\operatorname{span}\left\{P_{1}, \cdots, P_{j-1}, P_{j+1}, \cdots, P_{q}\right\}$ et $Q_{*}^{\overline{P_{j}}}$ la séquence de co-distributions définie en (1.35) initialisée par $\operatorname{span}\left\{\overline{P_{j}}\right\}$.

En d'autres termes, la condition $(i)$ traduit le fait qu'il est possible de synthétiser des résidus à partir d'une fonction non linéaire des sorties du système. La condition (ii) signifie qu'il n'existe pas de composantes du défaut $w(t)$ qui pourraient :

- ne pas être perçues par le résidu. Le résidu est formé par $\chi^{\overline{P_{j}}}(\bullet)$; le fait que toute composante de $w(t)$ doit influencer le résidu se traduit par une intersection vide entre le sous-espace d'état non observable par le résidu et le défaut.

- être masquées par un autre défaut. Nous rappelons que $Q_{*}^{\overline{P_{j}}}$ est appelée co-distribution d'observabilité (Observability Co-distribution Algorithm, [13] et [14]) de $\left(S_{*}^{\overline{P_{j}}}\right)^{\perp}$ soit : 
$Q_{*}^{\overline{P_{j}}}=$ o.c.a. $\left(\left(S_{*}^{\overline{P_{j}}}\right)^{\perp}\right) \subseteq\left(S_{*}^{\overline{P_{j}}}\right)^{\perp} \cdot Q_{*}^{\overline{P_{j}}}$ représente le sous-espace d'état observable insensible à tous les défauts sauf à $w_{j}(t)$.

La section suivante a pour objectif d'améliorer (en deux points) les conditions d'existence d'une solution au problème posé par la détection et l'isolation de défauts. Nous déterminons dans quelle mesure un ensemble de résidus permet la détection et l'isolation des défauts et il est proposé une méthode systématique assurant la synthèse de ces résidus.

Pour ce deuxième point, il est montré qu'un ensemble de défauts peut être détecté et isolé à l'aide de résidus sensibles à plusieurs d'entre eux. Cependant cette proposition ne peut être appliquée que sous l'hypothèse de non apparition simultanée des défauts.

\subsubsection{Proposition de nouvelles conditions de détection et d'isolation de défauts}

Pour mettre en valeur nos travaux, nous utilisons la distribution $S_{m *}^{P_{j}}((2.41)$ et $(2.53))$ pour mener à bien l'analyse structurelle. Une étude plus restrictive à l'aide de la distribution $S_{*}^{P_{j}}$ (définie par l'équation (1.34)) est cependant tout à fait possible [41].

L'analyse structurelle est basée sur l'étude des intersections et inclusions des sous-espaces d'états représentés par les deux distributions $S_{m *}^{P_{j}}$ et $C_{*}^{P_{j}}$ :

- $C_{*}^{P_{j}}$ est le plus grand sous-espace d'état sensible au défaut sans utiliser d'injection de sortie,

- $S_{m *}^{P_{j}}$ est le plus petit sous-espace d'état sensible au défaut en utilisant une injection de sortie.

Une interprétation de cette distinction est que l'injection de sortie ne stoppe pas la propagation du défaut mais permet d'en estimer l'effet. L'objet de la distribution $S_{m *}^{P_{j}}$ est de définir quel est le sous-espace d'état, sensible au défaut $w_{j}(t)$, ne pouvant être traduit à l'aide de signaux connus. Il paraît alors évident que la plus grande dimension de ce sous-espace d'état ne peut être obtenue qu'en l'absence d'injection de sortie. Cependant, lors de l'analyse structurelle que nous développons, $C_{*}^{P_{j}}$ ne peut pas être considéré comme étant le sous-espace d'état affecté par le défaut $w_{j}(t)$. En effet, ce dernier peut se trouver réduit (au sens de sa dimension) par la présence d'une injection de sortie, mais en aucun cas augmenté. Cette précision justifie la distinction que nous effectuons entre les conditions nécessaires (avec $C_{*}^{P_{j}}$ ) et les conditions suffisantes (avec $S_{m *}^{P_{j}}$ ). En nous fondant sur le test d'inclusion (2.23), nous nous attachons dans un premier temps à définir les conditions de détectabilité des défauts.

\subsubsection{Conditions d'existence d'un filtre détecteur de défauts}

Nous introduisons la distribution $\Xi^{P}$ définie par :

$$
\Xi^{P}=\gamma_{o b s}-\left(C_{*}^{P}\right)^{\perp}
$$


Un défaut influe sur au moins une sortie si, et seulement si, $\Xi^{P} \neq\{0\}$. Ainsi, le vecteur des défauts est détectable si, et seulement si, les inégalités $\Xi^{P_{j}} \neq\{0\}$ sont vérifiées pour tout $j$.

Théorème 2.10 La condition précédente $\Xi^{P} \neq\{0\}$, équivalente à $\gamma_{\text {obs }} \nsubseteq\left(C_{*}^{P}\right)^{\perp}, \forall j$ est :

(i) une condition nécessaire et suffisante d'existence d'un filtre détecteur, si $w(t) \in \mathbb{R}$

(ii) une condition nécessaire d'existence d'un filtre détecteur, si $w(t) \in \mathbb{R}^{q}$ avec $q>1$

\section{Preuve}

(i) Le premier point de ce théorème est trivial :

si $w(t) \in \mathbb{R}$ alors le défaut $w(t)$ est scalaire. Ainsi, il suffit qu'une sortie soit sensible à ce défaut. Quant à la nécessité, elle provient du fait que $\left(C_{*}^{P}\right)^{\perp}$ représente le plus petit sous-espace d'état insensible au défaut. Ainsi, s'il y a sensibilité, il existe une distribution observable qui n'est pas incluse dans $\left(C_{*}^{P}\right)^{\perp}$.

(ii) Le second point est plus délicat à démontrer :

si $w(t) \in \mathbb{R}^{q}$ (avec $q>1$ ) et $\Xi^{P} \neq\{0\}$, nous ne savons pas si les $q$ composantes du vecteur $w(t)$ sont représentées dans $\Xi^{P} \neq\{0\}$. En effet, il est possible que le sous-espace d'état sensible à une (ou plusieurs) partie(s) du vecteur défaut soit inobservable. Dans ce cas cette partie (ou ces parties) n'est pas détectable et cette condition n'est pas suffisante. En revanche, pour les mêmes raisons que précédemment (i), elle reste nécessaire.

En remarque, nous précisons que dans le cas particulier des systèmes observables, $\left(\operatorname{dim}\left(\operatorname{span}\left\{\gamma_{o b s}\right\}\right)=n, \gamma_{\text {inobs }}=\{0\}\right)$, le vecteur des défauts est détectable si $C_{*}^{P_{j}} \neq\{0\}$, $\forall j$.

En supposant que chaque défaut $w_{j}(t)(\forall j \in\{1, \cdots, q\})$ affecte au moins une sortie, nous nous proposons d'étudier le problème de la localisation des défauts. Dans un premier paragraphe, nous montrons la manière d'aboutir à des conditions nécessaires à l'isolation des défauts. Des conditions suffisantes sont, quant à elles, énoncées dans un deuxième paragraphe.

\subsubsection{Conditions nécessaires à l'isolation des défauts}

La détectabilité de l'ensemble des défauts constitue une première condition nécessaire à leur isolation. Cependant, l’isolabilité des défauts nécessite des hypothèses supplémentaires. En effet, il est possible que plusieurs défauts agissent sur une seule et même sortie, ce qui a pour effet de ne pas les rendre isolables puisque leurs effets ne sont alors pas distinguables par la simple évaluation des sorties. Pour approfondir les conditions nécessaires à l'isolation des défauts, une analyse se fondant sur les travaux de J-P. Cassar et al [11], J-F. Magni [50] et de J.J. Gertler [31] est menée. Ainsi, si nous étudions le défaut $w_{j}(t)$ et considérons le plus 
grand sous-espace d'état sensible à ce défaut exprimé par la distribution $C_{*}^{P_{j}}$. L'objectif est de déterminer, à l'aide de tests d'inclusion (analyse structurelle), la distribution insensible au plus grand nombre d'autres défauts. A cette fin, nous considérons les distributions $S_{m *}^{P_{i}}$ qui traduisent, via une injection de sortie, les plus petits sous-espaces d'état sensibles aux défauts $w_{i}(t)$. Naturellement, s'il existe une distribution incluse dans le sous-espace d'état insensible à tous les défauts sauf un, nous retrouvons le cas du F.P.R.G. Dans cette section, nous ne nous limitons pas à l'étude de ce cas puisque nous cherchons à déterminer une distribution sensible à un défaut particulier et devant être insensible au plus grand nombre d'autres défauts. Plus ce nombre est grand, plus grand est le nombre de défauts simultanément isolables. De plus, cette considération est en accord avec l'augmentation de la distance de Hamming ([11, 31]) qui est une mesure de la différence entre les signatures associées à chaque défaut.

Dans ce paragraphe, puisque la distribution $C_{*}^{P_{j}}$ majore en terme de dimension le sousespace d'état sensible au défaut, seules des conditions nécessaires mais non suffisantes sont déterminées pour l'isolation de défauts.

Nous précisons que toute distribution $\tau(x(t))$ telle que $\tau(x(t)) \subseteq\left(C_{*}^{P_{j}}\right)^{\perp}$ est nécessairement insensible au défaut $w_{j}(t)$ quelle que soit l'injection de sortie. En revanche, si $\tau(x(t)) \nsubseteq$ $\left(C_{*}^{P_{j}}\right)^{\perp}$, nous ne sommes pas capable de dire si cette distribution est sensible ou pas au défaut $w_{j}(t)$. Cependant, des conditions nécessaires peuvent être déclinées à l'aide de cette distribution.

Afin d'étudier les intersections des différents sous-espaces d'état, nous introduisons comme suit la matrice $\mathcal{A}^{P_{j}}$ associée au défaut $w_{j}(t)$ :

$$
\mathcal{A}^{P_{j}}=\left(\begin{array}{cccc}
\left(\mathcal{A}^{P_{j}}\right)_{1}^{1} & \left(\mathcal{A}^{P_{j}}\right)_{1}^{2} & \cdots & \left(\mathcal{A}^{P_{j}}\right)_{1}^{q} \\
\left(\mathcal{A}^{P_{j}}\right)_{2}^{1} & \left(\mathcal{A}^{P_{j}}\right)_{2}^{2} & \cdots & \left(\mathcal{A}^{P_{j}}\right)_{2}^{q} \\
\vdots & \vdots & \ddots & \vdots \\
\left(\mathcal{A}^{P_{j}}\right)_{2^{q-1}-1}^{1} & \left(\mathcal{A}^{P_{j}}\right)_{2^{q-1}-1}^{2} & \cdots & \left(\mathcal{A}^{P_{j}}\right)_{2^{q-1}-1}^{q}
\end{array}\right) \in \mathbb{R}^{\left(2^{q-1}-1\right) \times q}
$$

où $(\square)_{i}^{k}$ est l'élément de la $i^{\mathrm{e}}$ ligne et de la $k^{\mathrm{e}}$ colonne de la matrice $\square$. Dans le cas particulier d'une distribution (resp. co-distribution), l'indice $k$ (resp. $i$ ) n'est pas nécessaire.

La matrice $\mathcal{A}^{P_{j}}$ qui est composée de $2^{q-1}-1$ lignes et $q$ colonnes permet de tester toutes les combinaisons d'intersection entre les sous-espaces $\left(S_{m *}^{P_{k}}\right)^{\perp}$ avec $k \neq j$. Les résultats de ces tests sont traduits à l'aide de " 0 " et de "1" et forment ainsi une matrice binaire $\left(\mathcal{A}^{P_{j}}\right)_{i}^{k} \in\{0,1\}, \forall(i, j, k)$. Bien sûr, certains tests sont obsolètes au vu des résultats déjà obtenus.

Afin de mieux comprendre la méthode de construction de la matrice $\mathcal{A}^{P_{j}}$, nous proposons deux exemples. 


\section{Exemple 1}

Le cas "trois défauts" est considéré. Nous nous intéressons à l'élaboration de la matrice $\mathcal{A}^{P_{2}}$ associée au défaut $w_{2}(t)$ pondéré par $P_{2}$. Dans ce cas particulier, la matrice (2.72) est composée de 3 colonnes et $2^{2}-1=3$ lignes et s'écrit :

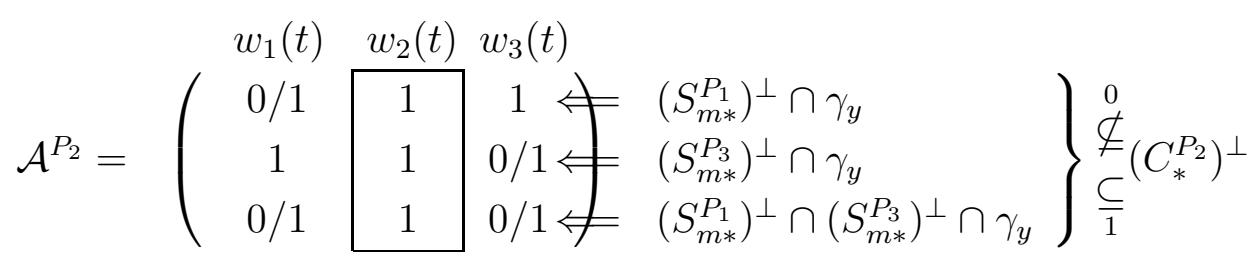

où le terme "0/1" est égal à " 1 " si l'inclusion est satisfaite et " 0 " sinon.

Nous rappelons que le fait d'étudier le défaut $w_{2}(t)$ signifie que nous cherchons une distribution sensible à $w_{2}(t)$. C'est pourquoi seule la non inclusion dans $\left(C_{*}^{P_{2}}\right)^{\perp}$ est recherchée et

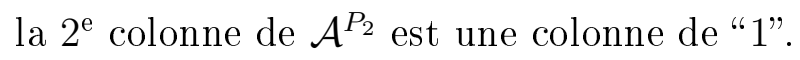

De plus, comme l'objectif de cette étude est la génération de résidus assurant l'isolation de l'ensemble des défauts, une contrainte supplémentaire doit être ajoutée : une solution (ligne binaire de la matrice notée $\left.\left(\mathcal{A}^{P_{j}}\right)_{i}\right)$ ne peut être considérée que s'il existe une distribution associée $y$-reconstructible. Ceci se traduit par la suite d'intersections avec $\gamma_{y}$ définie en $(2.4)$.

\section{Exemple 2}

Le cas "quatre défauts" est illustré. Nous nous intéressons à l'élaboration de la matrice $\mathcal{A}^{P_{3}}$ dédiée au défaut $w_{3}(t)$ pondéré par $P_{3}$. Dans ce cas particulier, la matrice (2.72) est composée de 4 colonnes et $2^{3}-1=7$ lignes et s'écrit :

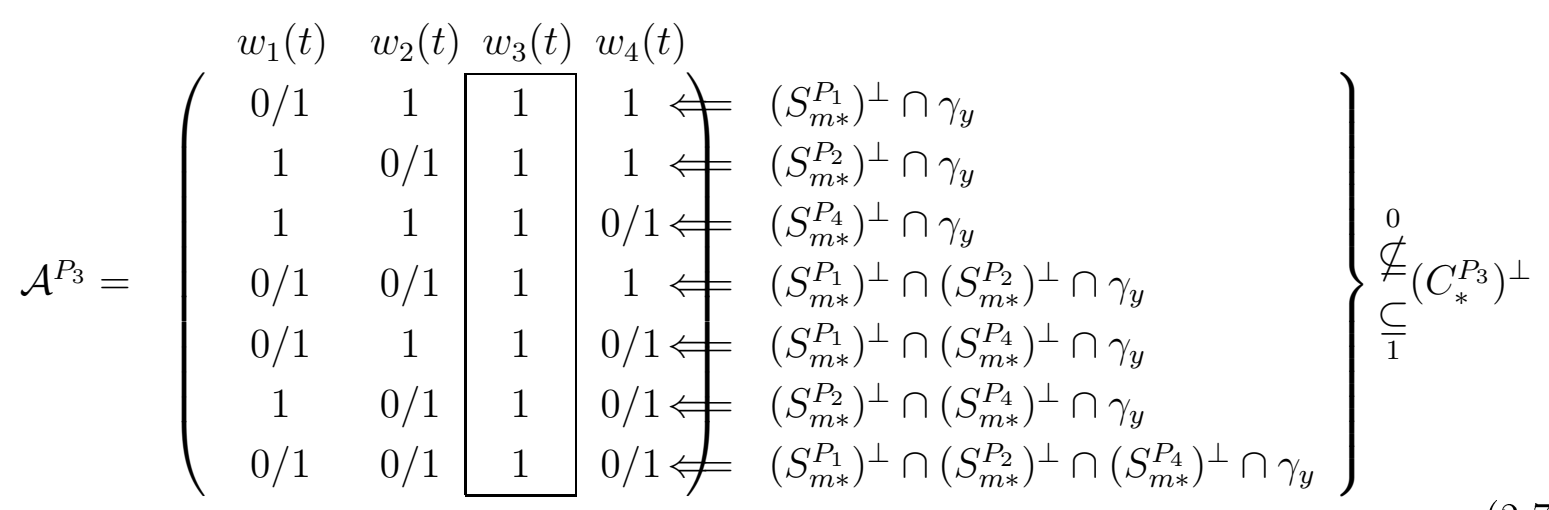


A cette fin, nous cherchons les lignes de la matrice (2.72) ayant un nombre maximal d'éléments égaux à " 0 ". Une fois les matrices $\mathcal{A}^{P_{j}}$ construites, nous déterminons les distributions sensibles à $w_{j}(t)$ et insensibles au plus grand nombre d'autres défauts.

Si $\beta j$ correspond à la valeur minimale du critère suivant :

$$
\beta j=\min _{i}\left(\sum_{k=1}^{q}\left(\mathcal{A}^{P_{j}}\right)_{i}^{k}\right)
$$

alors un nouvel ensemble noté $\mathcal{B}^{P_{j}}$ est défini :

$$
\mathcal{B}^{P_{j}}=\left(\begin{array}{c}
\left(\mathcal{B}^{P_{j}}\right)_{1} \\
\left(\mathcal{B}^{P_{j}}\right)_{2} \\
\vdots \\
\left(\mathcal{B}^{P_{j}}\right)_{\epsilon_{P_{j}}}
\end{array}\right) \in \mathbb{R}^{\epsilon_{P_{j}} \times q}
$$

où chaque élément $\left(\mathcal{B}^{P_{j}}\right)_{i}$ correspond à une ligne de $\mathcal{A}^{P_{j}}$ et est tel que $\sum_{k=1}^{q}\left(\mathcal{B}^{P_{j}}\right)_{i}^{k}=\beta j$.

Afin de déterminer les conditions d'isolation de l'ensemble des défauts, nous regroupons les $q$ matrices $\mathcal{B}^{P_{j}}$ au sein d'une seule et même matrice $\mathcal{A}$ de la façon suivante :

$$
\mathcal{A}=\left(\begin{array}{c}
\mathcal{B}^{P_{1}} \\
\mathcal{B}^{P_{2}} \\
\vdots \\
\mathcal{B}^{P_{q}}
\end{array}\right) \in \mathbb{R}^{\left.\left(\sum_{j=1}^{q} \epsilon_{P_{j}}\right)\right) \times q}
$$

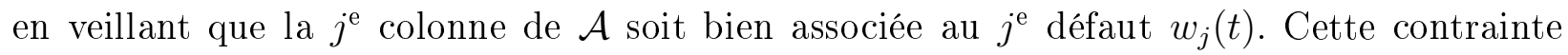
implique que les séquences de test d'inclusion visant la synthèse des matrices $\mathcal{B}^{P_{j}}$ pour $j=\{1, \cdots, q\}$ soient ordonnées.

Suite à la définition de la matrice (2.77), nous proposons le théorème suivant :

Théorème 2.11 Si le rang de la matrice binaire $\mathcal{A}$ est strictement inférieur à la dimension $q$ du défaut, c'est-à-dire si $\operatorname{rang}(\mathcal{A})<\operatorname{dim}(w(t))=q$ alors tous les défauts ne sont pas isolables.

\section{Preuve}

Nous rappelons que l'étude considère les distributions représentées par $C_{*}^{P_{j}}$. Celles-ci majorent le sous-espace sensible à un défaut $w_{j}(t)$ modulo une injection de sortie. $C^{\prime}$ 'st pourquoi nous avons $S_{m *}^{P_{j}} \subseteq S_{*}^{P_{j}} \subseteq C_{*}^{P_{j}}$ ou encore de manière équivalente $\left(C_{*}^{P_{j}}\right)^{\perp} \subseteq\left(S_{*}^{P_{j}}\right)^{\perp} \subseteq\left(S_{m *}^{P_{j}}\right)^{\perp}$. $\left(C_{*}^{P_{j}}\right)^{\perp}$ est donc le plus petit sous-espace d'état insensible au défaut $w_{j}(t)$.

La matrice $\mathcal{A}$ est synthétisée dans le cadre de la recherche du sous-espace d'état sensible à un défaut $w_{j}(t)$ et insensible au plus grand nombre d'autres défauts pour $j=\{1, \cdots, q\}$. 
Dans ce théorème, nous considérons qu'une distribution est sensible au défaut $w_{j}(t)$ si elle n'appartient pas à $\left(C_{*}^{P_{j}}\right)^{\perp}$ bien que celle-ci minore la réalité. Nous nous plaçons donc dans le meilleur des cas car il est fort probable que le sous-espace d'état insensible au défaut $w_{j}(t)$ soit plus grand que $\left(C_{*}^{P_{j}}\right)^{\perp}$.

Cependant, s'il n'existe pas de distributions non incluses dans $\left(C_{*}^{P_{j}}\right)^{\perp}$ alors il n'en existe pas (cf. jeux d'inclusion). Dans ce sens, il s'agit d'une condition nécessaire à l'isolation car si le rang de la matrice $\mathcal{A}$ n'excède pas $q-1$, ce ne sera jamais possible. Or le rang de la matrice $\mathcal{A}$ se veut le reflet du nombre de signatures différentes des défauts en terme de sensibilité sur les résidus. Ainsi s'il n'existe pas plus de $q-1$ signatures différentes, l'ensemble des composantes du vecteur défaut ne pourra pas être isolé.

La condition de rang définie dans ce paragraphe doit être testée avant toute autre étude. Elle constitue en effet une condition nécessaire à la localisation des défauts. Le paragraphe suivant propose en complément des conditions suffisantes à la résolution d'un tel problème. Celles-ci se fondent non plus sur la plus grande distribution sensible à un défaut, mais sur la plus petite par un choix optimal de l'injection de sortie.

\subsubsection{Conditions suffisantes à l'isolation des défauts}

A l'opposé du paragraphe précédent, nous minorons le sous-espace d'état sensible à un défaut par $S_{m *}^{P_{j}}$. En effet, toute distribution $\tau(x(t))$ telle que $\tau(x(t)) \nsubseteq\left(S_{m *}^{P_{j}}\right)^{\perp}$ est nécessairement sensible au défaut $w_{j}(t)$ quelle que soit l'injection de sortie.

Nous précisons que dans le cadre du calcul de $S_{m *}^{P_{j}}$, il est possible de découpler totalement le défaut $\left(S_{m *}^{P_{j}}=\{0\}\right)$. Ainsi nous distinguons deux cas lors de la synthèse de la matrice binaire $\mathcal{A}_{m}^{P_{j}}$ qui exprime les sensibilités de l'état au défaut $w_{j}(t)$ :

$\underline{\text { premier cas : } S_{m *}^{P_{j}}=\{0\}}$

Bien qu'il n'est pas judicieux de découpler totalement le défaut $w_{j}(t)$ dans dans le cadre de l'isolation, dans un contexte général nous traitons ce cas. $\mathcal{A}_{m}^{P_{j}}$ est défini identiquement à $\mathcal{A}^{P_{j}}$ mais en considérant la distribution $S_{m *}^{P_{j}}$ à la place de $\left(C_{*}^{P_{j}}\right)^{\perp}$. Dans ce cas la $j^{\mathrm{e}}$ colonne de la matrice $\mathcal{A}_{m}^{P_{j}}$ n'est plus une colonne de "1" mais plutôt de " 0 ", car aucune distribution de l'espace d'état n'est sensible à $w_{j}(t)$. Nous cherchons donc la distribution (non nulle) insensible au plus grand nombre de défauts.

deuxième cas : $S_{m *}^{P_{j}} \neq\{0\}$

D'une manière analogue à $\mathcal{A}^{P_{j}}, \mathcal{A}_{m}^{P_{j}}$ est définie avec $\left(S_{m *}^{P_{j}}\right)^{\perp}$ à la place de $\left(C_{*}^{P_{j}}\right)^{\perp}$. 
En menant les mêmes calculs que précédemment, avec pour base de réflexion la matrice $\mathcal{A}_{m}^{P_{j}}$, nous obtenons la matrice de synthèse suivante :

$$
\mathcal{A}_{m}=\left(\begin{array}{c}
\mathcal{B}_{m}^{P_{1}} \\
\mathcal{B}_{m}^{P_{2}} \\
\vdots \\
\mathcal{B}_{m}^{P_{q}}
\end{array}\right) \in \mathbb{R}^{\left(\sum_{j=1}^{q} \epsilon_{P_{j}}\right) \times q}
$$

Nous proposons le théorème suivant :

Théorème 2.12 Si le rang de la matrice binaire $\mathcal{A}_{m}$ est égal à la dimension du défaut, c'est-à-dire si $\operatorname{rang}\left(\mathcal{A}_{m}\right)=\operatorname{dim}(w(t))=q$ alors tous les défauts sont isolables.

\section{Preuve}

Si le rang de la matrice $\mathcal{A}_{m}$ est égal à q, alors il existe q résidus possédant des sensibilités différentes aux défauts. Il est alors possible de distinguer l'ensemble des défauts.

Contrairement au théorème 2.11, la synthèse de la matrice $\mathcal{A}_{m}$ est réalisée à l'aide de la distribution $S_{m *}^{P_{j}}$ pour $j=\{1, \cdots, q\}$. Or comme nous l'avons montré dans la section précédente, il s'agit du plus petit sous-espace d'état sensible au défaut $w_{j}(t)$. Par conséquent $\left(S_{m *}^{P_{j}}\right)^{\perp}$ est le plus grand sous-espace d'état insensible au défaut $w_{j}(t)$.

Ainsi, nous savons que cette distribution minore la réalité car l'objectif est non pas de minimiser l'effet du défaut $w_{j}(t)$ mais plutôt celui du plus grand nombre des autres défauts. Nous nous situons donc dans le pire des cas, ce qui explique le caractère suffisant du théorème.

Contrairement au découplage des systèmes, lors de l'analyse du défaut particulier $w_{j}(t)$ menant à la détermination de la matrice $\mathcal{A}_{m}^{P_{j}}$ puis d'une ligne de $\mathcal{A}_{m}$, l'estimation du défaut peut être préjudiciable pour la localisation. En effet, sous cette hypothèse, la colonne $j$ de la matrice $\mathcal{A}_{m}$ est une colonne de "0". Nous rappelons qu'un défaut estimable est associé à un sous-espace d'état dans sa totalité insensible à ce défaut. Dans ces termes comment pourrait-t-on détecter ce défaut? C'est pourquoi nous conseillons lors de l'étude particulière du défaut $w_{j}(t)$ et donc menant à la synthèse de la matrice $\mathcal{A}_{m}^{P_{j}}$ d'inhiber l'étape d'estimation du défaut. Par conséquent, tout en utilisant une injection de sortie généralisée avec $\left(\bar{S}_{m 1}^{P_{x}}=\operatorname{span}\left\{P_{x}\right\}(2.53)\right)$, nous assurons une diagonale de "1". De plus dans tous les cas, en remarquant que $\operatorname{span}\left\{P_{x}\right\} \subseteq S_{m *}^{P_{x}} \subseteq S_{*}^{P_{x}}$ est toujours vérifié, les conditions d'isolations qui en sont déduites seront nécessairement moins restrictives que celles énumérées en (2.69) et (2.70).

Inhiber l'étape d'estimation du défaut lors de l'établissement des conditions suffisantes à l'isolation des défauts, ne remet évidemment pas en cause les résultats de découplage assurés par cette étape (paragraphe 2.3.3.1). 
Suite à l'établissement des théorèmes 2.11 et 2.12, il nous semble important de préciser la démarche à suivre lorsque l'isolation de défauts est l'objet d'une étude.

\subsubsection{Méthodologie}

Lors de l'analyse de la géométrie d'un système non linéaire, dans le but d'en réaliser le diagnostic, il semble important de valider les différentes étapes suivantes.

Dans un premier temps, rappelons que diagnostiquer un système c'est détecter et localiser l'ensemble des défauts pouvant agir sur ce dernier. Par hypothèse de travail, en ce qui nous concerne, les défauts sont préalablement identifiés.

Nous définissons précisément la démarche, à l'aide d'étapes à valider :

(1) la première étape est sans nulle doute la détectabilité de l'ensemble des défauts. C'est-àdire que chaque défaut $w_{j}(t)$ doit avoir un effet sur au moins une sortie $\left(\gamma_{o b s} \nsubseteq\left(C_{*}^{P_{j}}\right)^{\perp}, \forall j\right)$.

(2) la deuxième étape est le test des conditions nécessaires à l'isolation des défauts. C'està-dire la vérification du théorème 2.11 .

(3) la troisième étape est le test des conditions suffisantes à l'isolation des défauts. C'est-àdire la vérification du théorème 2.12 .

Si toutes ces étapes sont validées alors un vecteur de résidus peut être généré par (en rappelant que chacune des distributions retenue lors de l'étude se veut $y$-reconstructible) :

$$
r(t)=\chi\left(y_{x}(t)\right)-\chi\left(y_{z}(t)\right)=\chi \circ h(x(t))-\chi \circ h(z(t))
$$

et pour conclure cette étude, la matrice $\mathcal{A}_{m}$ peut s'interpréter comme la table de signature TAB 2.1 (en considérant inhibée l'étape d'estimation des défauts). Il semble alors évident que certaines configurations (en terme de sensibilité) ne permettent pas l'isolation des défauts s'ils apparaissent simultanément.

En revanche, si les étapes (1) et (2) sont validées sans que la troisième le soit, alors nous ne pouvons pas conclure. C'est-à-dire que nous ne sommes pas en mesure à l'aide des conditions de rang d'affirmer ou d'infirmer si les défauts sont isolables. Nous ajoutons que si l'étape (3) est validée, (2) l'est aussi. Et si l'étape (2) n'est pas validée (3) ne peut l'être. Ces deux dernières remarques sont triviales en précisant que $S_{m *}^{P_{j}} \nsubseteq C_{*}^{P_{j}}$.

Nous proposons l'étude de deux exemples académiques afin de mieux appréhender les 3 étapes que nous venons de décrire. 


\begin{tabular}{|c|c|c|c|c|}
\hline $\begin{array}{ll}\text { Résid us } & \text { Défauts } \\
\end{array}$ & $w_{1}$ & $w_{2}$ & $\cdots$ & $w_{\mathbf{q}}$ \\
\hline \multirow{3}{*}{$r_{1}$} & \multirow{3}{*}{ sensible à to $w_{1}$} & $\begin{array}{c}\text { insensible à } w_{2} \text { si }\left(A_{m}^{P_{1}}\right)_{1}^{1}=0 \\
\text { sinon sensible }\end{array}$ & \multirow{3}{*}{$\cdots$} & $\begin{array}{c}\text { insensible à } w_{\mathbf{q}} \text { si }\left(A_{m}^{P_{1}}\right)_{1}^{q}=0 \\
\text { sinon sensible }\end{array}$ \\
\hline & & & & \\
\hline & & $\begin{array}{c}\text { insensible à } w_{2} \text { si }\left(A_{m}^{p_{1}}\right)_{\epsilon_{p_{1}}}^{1}=0 \\
\text { sinon sensible }\end{array}$ & & $\begin{array}{c}\text { insensible à } w_{\mathbf{q}} \text { si }\left(A_{m}^{P_{1}}\right)_{\epsilon_{P_{1}}^{q}}^{q}=0 \\
\text { sinon sensible }\end{array}$ \\
\hline \multirow{3}{*}{$r_{2}$} & $\begin{array}{c}\text { insensible à } w_{1} \text { si }\left(A_{m}^{P_{2}}\right)_{1}^{1}=0 \\
\text { sinon sensible }\end{array}$ & \multirow{3}{*}{ sensible à $w_{2}$} & & $\begin{array}{c}\text { insensible à } w_{\mathbf{q}} \text { si }\left(A_{m}^{P_{2}}\right)_{1}^{q}=0 \\
\text { sinon sensible }\end{array}$ \\
\hline & & & & \\
\hline & $\begin{array}{c}\text { insensible à } w_{1} \text { si }\left(A_{m}^{P_{2}}\right)_{\epsilon_{P_{2}}}^{1}=0 \\
\text { sinon sensible }\end{array}$ & & & $\begin{array}{c}\text { insensible à } w_{\mathbf{q}} \text { si }\left(A_{m}^{P 2}\right)_{\epsilon_{P_{2}}}^{q}=0 \\
\text { sinon sensible }\end{array}$ \\
\hline$\vdots$ & . & & & \\
\hline \multirow{3}{*}{$r_{q}$} & $\begin{array}{c}\text { insensible à } w_{1} \text { si }\left(A_{m}^{P_{q}}\right)_{1}^{1}=0 \\
\text { sinon sensible }\end{array}$ & $\begin{array}{c}\text { insensible à } w_{2} \text { si }\left(A_{m}^{P_{q}}\right)_{1}^{2}=0 \\
\text { sinon sensible }\end{array}$ & \multirow{3}{*}{$\cdots$} & \multirow{3}{*}{ sensible à $w_{\mathbf{q}}$} \\
\hline & $\vdots$ & $\vdots$ & & \\
\hline & $\begin{array}{c}\text { insensible à } w_{1} \text { si }\left(A_{m}^{P q}\right)_{\epsilon_{P q}}^{1}=0 \\
\text { sinon sensible }\end{array}$ & $\begin{array}{c}\text { insensible à } w_{2} \text { si }\left(A_{m}^{P q}\right)_{\epsilon_{P_{q}}^{2}}^{2}=0 \\
\text { sinon sensible }\end{array}$ & & \\
\hline
\end{tabular}

TAB. 2.1: Table de signatures

\subsubsection{Exemples académiques}

\subsubsection{Exemple 1}

Le système considéré est non linéaire affine en la commande et modélisé comme suit (cf. [25]) :

$$
\Sigma_{N L 1}:\left\{\begin{array}{l}
\dot{x}(t)=\left(\begin{array}{c}
x_{1}(t) x_{4}(t) \\
x_{3}(t)\left(1-x_{4}(t)\right) \\
0 \\
0
\end{array}\right)+\left(\begin{array}{cc}
0 & 0 \\
0 & 0 \\
0 & x_{1}(t) \\
1 & 0
\end{array}\right)\left(\begin{array}{l}
u_{1}(t)+w_{1}(t) \\
u_{2}(t)+w_{2}(t)
\end{array}\right) \\
y_{x}(t)=\left(\begin{array}{l}
x_{1}(t) \\
x_{3}(t)
\end{array}\right)
\end{array}\right.
$$

où $x(t) \in \mathbb{R}^{4}, u(t) \in \mathbb{R}^{2}, y_{x}(t) \in \mathbb{R}^{2}$ et $w(t) \in \mathbb{R}^{2}$ sont respectivement les états, commandes, sorties et défauts du système.

Nous calculons les différentes distributions définies au sein de ce chapitre : 


$$
\begin{array}{ll}
C_{*}^{P_{1}}=\operatorname{span}\left\{\left(\begin{array}{l}
0 \\
0 \\
0 \\
1
\end{array}\right),\left(\begin{array}{c}
-x_{1}(t) \\
x_{3}(t) \\
0 \\
0
\end{array}\right),\left(\begin{array}{c}
0 \\
x_{1}(t) \\
x_{1}(t) \\
0
\end{array}\right),\left(\begin{array}{c}
0 \\
x_{1}(t)+x_{2}(t) \\
x_{1}(t) \\
0
\end{array}\right)\right\} & C_{*}^{P_{2}}=\operatorname{span}\left\{\left(\begin{array}{c}
0 \\
0 \\
x_{1}(t) \\
0
\end{array}\right),\left(\begin{array}{c}
0 \\
-x_{1}(t)\left(1-x_{4}(t)\right) \\
x_{1}(t) x_{4}(t) \\
0
\end{array}\right)\right\} \\
\left(C_{*}^{P_{1}}\right)^{\perp}=\{0\} & \left(C_{*}^{P_{2}}\right)^{\perp}=\operatorname{span}\left\{\left(\begin{array}{l}
1 \\
0 \\
0 \\
0
\end{array}\right),\left(\begin{array}{l}
0 \\
0 \\
0 \\
1
\end{array}\right)\right\} \\
S_{*}^{P_{1}}=\operatorname{span}\left\{\left(\begin{array}{l}
0 \\
0 \\
0 \\
1
\end{array}\right),\left(\begin{array}{c}
-x_{1}(t) \\
x_{3}(t) \\
0 \\
0
\end{array}\right)\right\} & S_{*}^{P_{2}}=\operatorname{span}\left\{\left(\begin{array}{c}
0 \\
0 \\
x_{1}(t) \\
0
\end{array}\right)\right\} \\
S_{m *}^{P_{1}}=\{0\} & S_{m^{2}}^{P_{2}}=\{0\} \\
\left(S_{*}^{P_{1}}\right)^{\perp}=\operatorname{span}\left\{\left(\begin{array}{l}
0 \\
0 \\
1 \\
0
\end{array}\right),\left(\begin{array}{l}
x_{3}(t) \\
x_{1}(t) \\
0 \\
0
\end{array}\right)\right\} \quad\left(S_{*}^{P_{2}}\right)^{\perp}=\operatorname{span}\left\{\left(\begin{array}{l}
1 \\
0 \\
0 \\
0
\end{array}\right),\left(\begin{array}{l}
0 \\
1 \\
0 \\
0
\end{array}\right),\left(\begin{array}{l}
0 \\
0 \\
0 \\
1
\end{array}\right)\right\} \\
\left(S_{m *}^{P_{1}}\right)^{\perp}=\operatorname{span}\left\{\left(\begin{array}{l}
1 \\
0 \\
0 \\
0
\end{array}\right),\left(\begin{array}{l}
0 \\
1 \\
0 \\
0
\end{array}\right),\left(\begin{array}{l}
0 \\
0 \\
1 \\
0
\end{array}\right),\left(\begin{array}{l}
0 \\
0 \\
0 \\
1
\end{array}\right)\right\}
\end{array}
$$

Les dimensions des distributions $S_{*}^{P_{1}}$ et $S_{*}^{P_{2}}$ sont diminuées lors du calcul de $S_{m *}^{P_{1}}$ et $S_{m *}^{P_{2}}$ car il est possible d'estimer les défauts $w_{1}(t)$ et $w_{2}(t)$ à partir d'une combinaison de sorties. En effet, nous vérifions facilement que $w_{1}(t)=\frac{y_{x 1}^{(2)}(t) y_{x 1}(t)-\left(y_{x 1}^{(1)}(t)\right)^{2}}{y_{x 1}^{2}(t)}-u_{1}(t)$ et $w_{2}(t)=\left(y_{x 2}^{(1)}(t)-\right.$ $\left.y_{x 1}(t) u_{2}(t)\right) / y_{x 1}(t)$.

\section{Conditions nécessaires (théorème 2.11)}

En nous aidant des calculs précédents, nous vérifions les non-inclusions suivantes :

$$
\begin{aligned}
& \left(S_{*}^{P_{2}}\right)^{\perp} \cap \gamma_{y} \nsubseteq\left(C_{*}^{P_{1}}\right)^{\perp} \\
& \left(S_{*}^{P_{1}}\right)^{\perp} \cap \gamma_{y} \nsubseteq\left(C_{*}^{P_{2}}\right)^{\perp}
\end{aligned} \quad \Rightarrow \mathcal{A}=\left(\begin{array}{ll}
1 & 0 \\
0 & 1
\end{array}\right) \Rightarrow \operatorname{rang}(\mathcal{A})=2
$$

De plus puisque $S_{m *}^{P_{j}} \subseteq S_{*}^{P_{j}}$, ce qui suit est évident :

$$
\begin{aligned}
& \left(S_{m *}^{P_{2}}\right)^{\perp} \cap \gamma_{y} \nsubseteq\left(C_{*}^{P_{1}}\right)^{\perp} \\
& \left(S_{m *}^{P_{1}}\right)^{\perp} \cap \gamma_{y} \nsubseteq\left(C_{*}^{P_{2}}\right)^{\perp}
\end{aligned} \quad \Rightarrow \quad \mathcal{A}=\left(\begin{array}{ll}
1 & 0 \\
0 & 1
\end{array}\right) \Rightarrow \operatorname{rang}(\mathcal{A})=2
$$

En conclusion, nous pouvons affirmer que les conditions nécessaires à l'isolation de défaut sont vérifiées, tant avec une injection de sortie restreinte (analyse structurelle avec $S_{*}^{P_{j}}$ ) que généralisée (analyse structurelle avec $S_{m *}^{P_{j}}$ ). Il est possible d'isoler l'ensemble des défauts si les conditions du théorème 2.12 sont également satisfaites. 


\section{Conditions suffisantes (théorème 2.12)}

Nous testons les inclusions suivantes :

$$
\begin{aligned}
& \left(S_{*}^{P_{2}}\right)^{\perp} \cap \gamma_{y} \nsubseteq\left(S_{*}^{P_{1}}\right)^{\perp} \\
& \left(S_{*}^{P_{1}}\right)^{\perp} \cap \gamma_{y} \nsubseteq\left(S_{*}^{P_{2}}\right)^{\perp}
\end{aligned} \quad \Rightarrow \quad \mathcal{A}_{m}=\left(\begin{array}{ll}
1 & 0 \\
0 & 1
\end{array}\right) \Rightarrow \operatorname{rang}\left(\mathcal{A}_{m}\right)=2
$$

et,

$$
\begin{aligned}
& \left(S_{m *}^{P_{2}}\right)^{\perp} \cap \gamma_{y} \nsubseteq S_{m *}^{P_{1}} \\
& \left(S_{m *}^{P_{1}}\right)^{\perp} \cap \gamma_{y} \nsubseteq S_{m *}^{P_{2}}
\end{aligned} \quad \Rightarrow \quad \mathcal{A}_{m}=\left(\begin{array}{ll}
0 & 0 \\
0 & 0
\end{array}\right) \Rightarrow \operatorname{rang}\left(\mathcal{A}_{m}\right)=0
$$

Puisque $S_{m *}^{P_{1}}=\{0\}$ et $S_{m *}^{P_{2}}=\{0\}$, nous nous trouvons dans le premier cas énoncé paragraphe 2.4.2.3 et les conditions du théorème 2.12 ne sont pas satisfaites. En revanche en inhibant l'étape d'estimation lors du calcul de $S_{m *}^{P_{1}} \neq\{0\}$ et $S_{m *}^{P_{2}} \neq\{0\}$, nous nous trouvons dans le deuxième cas :

$$
\begin{aligned}
& \left(S_{m *}^{P_{2}}\right)^{\perp} \cap \gamma_{y} \nsubseteq\left(S_{m *}^{P_{1}}\right)^{\perp} \\
& \left(S_{m *}^{P_{1}}\right)^{\perp} \cap \gamma_{y} \subseteq\left(S_{m *}^{P_{2}}\right)^{\perp}
\end{aligned} \quad \Rightarrow \mathcal{A}_{m}=\left(\begin{array}{ll}
1 & 0 \\
1 & 1
\end{array}\right) \Rightarrow \operatorname{rang}\left(\mathcal{A}_{m}\right)=2
$$

En conclusion, nous sommes certains de pouvoir isoler les deux défauts $w_{1}(t)$ et $w_{2}(t)$ en utilisant une injection de sortie restreinte puisque les conditions suffisantes à l'isolation du défaut sont vérifiées.

En revanche, concernant l'injection de sortie généralisée, les conditions suffisantes ne sont pas vérifiées. Nous notons la diagonale de "0" de la matrice $\mathcal{A}_{m}$ équation (2.84). Nous ne sommes donc pas en mesure de conclure.

Cette caractéristique était prévisible car le découplage des défauts $w_{1}(t)$ et $w_{2}(t)$ est total ce qui implique qu'aucune partie du sous-espace d'état n'y est sensible. En nous interdisant toute possibilité de découplage total (étape d'estimation inhibée), nous obtenons les mêmes résultats qu'avec une injection de sortie restreinte.

\subsubsection{Exemple 2}

Le système considéré est modélisé comme suit :

$$
\Sigma_{N L 2}:\left\{\begin{array}{l}
\dot{x}(t)=\left(\begin{array}{c}
x_{1}(t) x_{4}(t) \\
x_{3}(t)\left(1-x_{4}(t)\right) \\
0 \\
0
\end{array}\right)+\left(\begin{array}{cc}
0 & 0 \\
0 & 0 \\
0 & x_{1}(t) \\
1 & 0
\end{array}\right)\left(\begin{array}{l}
u_{1}(t)+w_{1}(t) \\
u_{2}(t)+w_{2}(t)
\end{array}\right) \\
y_{x}(t)=\left(\begin{array}{l}
x_{1}(t) \\
x_{2}(t)
\end{array}\right)
\end{array}\right.
$$

où $x(t) \in \mathbb{R}^{4}, u(t) \in \mathbb{R}^{2}, y_{x}(t) \in \mathbb{R}^{2}$ et $w(t) \in \mathbb{R}^{2}$ sont respectivement les états, commandes, sorties et défauts du système non linéaire affine en la commande (2.85). 
Nous insistons sur le changement du vecteur de sortie en comparant avec le système (2.79). Nous illustrons par cet exemple l'influence de $\gamma_{y}$ lors de l'écriture des matrices $\mathcal{A}$ et $\mathcal{A}_{m}$.

$$
\begin{aligned}
& C_{*}^{P_{1}}=\operatorname{span}\left\{\left(\begin{array}{l}
0 \\
0 \\
0 \\
1
\end{array}\right),\left(\begin{array}{c}
-x_{1}(t) \\
x_{3}(t) \\
0 \\
0
\end{array}\right),\left(\begin{array}{c}
0 \\
x_{1}(t) \\
x_{1}(t) \\
0
\end{array}\right),\left(\begin{array}{c}
0 \\
x_{1}(t)+x_{2}(t) \\
x_{1}(t) \\
0
\end{array}\right)\right\} \quad C_{*}^{P_{2}}=\operatorname{span}\left\{\left(\begin{array}{c}
0 \\
0 \\
x_{1}(t) \\
0
\end{array}\right),\left(\begin{array}{c}
0 \\
-x_{1}(t)\left(1-x_{4}(t)\right) \\
x_{1}(t) x_{4}(t) \\
0
\end{array}\right)\right\} \\
& \left(C_{*}^{P_{1}}\right)^{\perp}=\{0\} \\
& S_{*}^{P_{1}}=\operatorname{span}\left\{\left(\begin{array}{l}
0 \\
0 \\
0 \\
1
\end{array}\right),\left(\begin{array}{c}
-x_{1}(t) \\
x_{3}(t) \\
0 \\
0
\end{array}\right)\right\} \\
& S_{*}^{P_{2}}=\operatorname{span}\left\{\left(\begin{array}{c}
0 \\
0 \\
x_{1}(t) \\
0
\end{array}\right),\left(\begin{array}{c}
0 \\
-x_{1}(t)\left(1-x_{4}(t)\right) \\
x_{1}(t) x_{4}(t) \\
0
\end{array}\right)\right\} \\
& S_{m *}^{P_{1}}=\operatorname{span}\left\{\left(\begin{array}{l}
0 \\
0 \\
0 \\
1
\end{array}\right)\right\} \\
& S_{m *}^{P_{2}}=\operatorname{span}\left\{\left(\begin{array}{c}
0 \\
0 \\
x_{1}(t) \\
0
\end{array}\right)\right\} \\
& \left(S_{*}^{P_{1}}\right)^{\perp}=\operatorname{span}\left\{\left(\begin{array}{l}
0 \\
0 \\
1 \\
0
\end{array}\right),\left(\begin{array}{c}
x_{3}(t) \\
x_{1}(t) \\
0 \\
0
\end{array}\right)\right\} \\
& \left(S_{*}^{P_{2}}\right)^{\perp}=\operatorname{span}\left\{\left(\begin{array}{l}
1 \\
0 \\
0 \\
0
\end{array}\right),\left(\begin{array}{l}
0 \\
0 \\
0 \\
1
\end{array}\right)\right\} \\
& \left(S_{m *}^{P_{1}}\right)^{\perp}=\operatorname{span}\left\{\left(\begin{array}{l}
1 \\
0 \\
0 \\
0
\end{array}\right),\left(\begin{array}{l}
0 \\
1 \\
0 \\
0
\end{array}\right),\left(\begin{array}{l}
0 \\
0 \\
1 \\
0
\end{array}\right)\right\} \\
& \left(C_{*}^{P_{2}}\right)^{\perp}=\operatorname{span}\left\{\left(\begin{array}{l}
1 \\
0 \\
0 \\
0
\end{array}\right),\left(\begin{array}{l}
0 \\
0 \\
0 \\
1
\end{array}\right)\right\} \\
& \left(S_{m^{*}}^{P_{2}}\right)^{\perp}=\operatorname{span}\left\{\left(\begin{array}{l}
1 \\
0 \\
0 \\
0
\end{array}\right),\left(\begin{array}{l}
0 \\
1 \\
0 \\
0
\end{array}\right),\left(\begin{array}{l}
0 \\
0 \\
0 \\
1
\end{array}\right)\right\}
\end{aligned}
$$

En commentaire de ces calculs, nous précisons que l'étape d'estimation est inhibée lors du calcul de $S_{m *}^{P_{1}}$ et $S_{m *}^{P_{2}}$. Les dimensions des sous-espaces d'état associés aux distributions $S_{m *}^{P_{1}}$ et $S_{m *}^{P_{2}}$ s'avèrent diminuées par rapport à $S_{*}^{P_{1}}$ et $S_{*}^{P_{2}}$.

\section{Conditions nécessaires (théorème 2.11)}

$$
\begin{aligned}
& \left(S_{*}^{P_{2}}\right)^{\perp} \cap \gamma_{y} \not \subset\left(C_{*}^{P_{1}}\right)^{\perp} \\
& \left(S_{*}^{P_{1}}\right)^{\perp} \cap \gamma_{y} \not \subseteq\left(C_{*}^{P_{2}}\right)^{\perp}
\end{aligned} \quad \Rightarrow \mathcal{A}=\left(\begin{array}{ll}
1 & 0 \\
0 & 1
\end{array}\right) \Rightarrow \operatorname{rang}(\mathcal{A})=2
$$

De plus, si nous considérons les distributions nouvellement définies,

$$
\begin{aligned}
& \left(S_{m *}^{P_{2}}\right)^{\perp} \cap \gamma_{y} \nsubseteq \nsubseteq\left(C_{*}^{P_{1}}\right)^{\perp} \\
& \left(S_{m *}^{P_{1}}\right)^{\perp} \cap \gamma_{y} \nsubseteq \subseteq\left(C_{*}^{P_{2}}\right)^{\perp}
\end{aligned} \quad \Rightarrow \mathcal{A}=\left(\begin{array}{ll}
1 & 0 \\
0 & 1
\end{array}\right) \Rightarrow \operatorname{rang}(\mathcal{A})=2
$$

Les conditions nécessaires à l'isolation des défauts sont vérifiées quel que soit le type de l'injection de sortie considérée (injection utilisant les signaux connus $y(t)$ ou $\Delta$ ). 


\section{Conditions suffisantes (théorème 2.12)}

$$
\begin{aligned}
& \left(S_{*}^{P_{2}}\right)^{\perp} \cap \gamma_{y} \subseteq\left(S_{*}^{P_{1}}\right)^{\perp} \\
& \left(S_{*}^{P_{1}}\right)^{\perp} \cap \gamma_{y} \nsubseteq\left(S_{*}^{P_{2}}\right)^{\perp}
\end{aligned} \quad \Rightarrow \quad \mathcal{A}_{m}=\left(\begin{array}{ll}
1 & 1 \\
0 & 1
\end{array}\right) \Rightarrow \operatorname{rang}\left(\mathcal{A}_{m}\right)=2
$$

et

$$
\begin{aligned}
& \left(S_{m *}^{P_{2}}\right)^{\perp} \cap \gamma_{y} \subseteq\left(S_{m *}^{P_{1}}\right)^{\perp} \\
& \left(S_{m *}^{P_{1}}\right)^{\perp} \cap \gamma_{y} \nsubseteq\left(S_{m *}^{P_{2}}\right)^{\perp}
\end{aligned} \quad \Rightarrow \mathcal{A}_{m}=\left(\begin{array}{ll}
1 & 1 \\
0 & 1
\end{array}\right) \Rightarrow \operatorname{rang}\left(\mathcal{A}_{m}\right)=2
$$

Nous insistons sur la ligne de "1" composant les matrices $\mathcal{A}_{m}$. Celle-ci est due au fait qu'il n'existe pas de distribution $y$-reconstructible sensible à $w_{2}(t)$ et insensible à $w_{1}(t)\left(\left(S_{m *}^{P_{2}}\right)^{\perp} \cap\right.$ $\gamma_{y} \subseteq\left(S_{m *}^{P_{1}}\right)^{\perp}$ alors que $\left.\left(S_{m *}^{P_{2}}\right)^{\perp} \nsubseteq\left(S_{m *}^{P_{1}}\right)^{\perp}\right)$.

Les conditions suffisantes à l'isolation des défauts sont tout de même satisfaites quel que soit le type de l'injection de sortie considérée. Nous pouvons conclure cette étude en affirmant que tous les défauts sont isolables.

\subsection{Conclusion}

En nous fondant sur les travaux de C. De Persis et A. Isidori qui sont rappelés et commentés, nous détaillons dans ce chapitre la problématique associée au calcul de la plus petite distribution invariante insensible à un défaut.

Il se fonde sur la modification de l'injection de sortie et plus particulièrement sur sa généralisation en terme de signaux la composant. Cette amélioration se perçoit à deux niveaux. Le premier est la définition du découplage total. Ceci est la conséquence d'une estimation de la perturbation. Le deuxième est la minimisation du sous-espace d'état sensible à un défaut, menant à l'augmentation non chiffrée mais démontrée de la borne inférieure. De plus, nous proposons une méthode intégrant des contraintes physiques dans le calcul du plus petit sous-espace insensible à un défaut.

Suite au calcul de ces distributions, une étude qualifiée d'analyse structurelle est présentée afin de déterminer les conditions nécessaires à l'isolation des défauts d'une part et suffisantes d'autre part. Cette étude est novatrice dans le sens où les conditions proposées lors de la résolution du F.P.R.G. ne sont pas toujours nécessaires. En effet, nous montrons que l'isolation de défauts peut être réalisée avec des signatures non diagonales.

Enfin, chaque résultat est illustré à l'aide d'exemples académiques permettant une mise en valeur des différents travaux effectués. 


\section{Chapitre 3}

\section{Synthèse de filtres non linéaires}

Plusieurs méthodes de découplage ont été proposées dans le chapitre précédent en considérant différentes formes d'injection de sortie.

Dans la première partie de ce chapitre, nous nous proposons d'étudier, pour chacune d'entre elles, une méthodologie menant à la détermination explicite de l'injection de sortie pour la synthèse de filtres.

De manière identique à la synthèse d'observateurs, le filtrage visant l'estimation de l'état réel du système nécessite l'étude de la convergence de ses estimations. Dans la deuxième partie nous proposons une étude novatrice permettant la preuve de la convergence des filtres de détection et localisation.

\subsection{Problématique}

Considérons le modèle du système suivant :

$$
\Sigma_{N L}:\left\{\begin{array}{l}
\dot{x}(t)=f_{0}(x(t))+\sum_{i=1}^{m} f_{i}(x(t)) u_{i}(t)+P(x(t)) w(t) \\
y_{x}(t)=h(x(t))
\end{array}\right.
$$

En supposant que l'une des méthodes proposées dans le chapitre précédent permet de restreindre l'effet d'une partie du défaut $w(t)$ à une partie du sous-espace d'état $x(t)$, nous définissons le difféomorphisme suivant :

$$
\tilde{x}(t)=\Phi(x(t))=\left(\begin{array}{c}
\Phi_{1}(x(t)) \\
--- \\
\Phi_{2}(x(t))
\end{array}\right)=\left(\begin{array}{c}
\tilde{x}_{1}(t) \\
--- \\
\tilde{x}_{2}(t)
\end{array}\right)
$$


où $\operatorname{span}\left\{d \tilde{x}_{1}^{T}\right\}=\operatorname{span}\left\{\left(\left(d \tilde{x}_{2}\right)^{\perp}\right)^{T}\right\}$.

Suite à l'application de cette transformation le système (3.1) s'écrit :

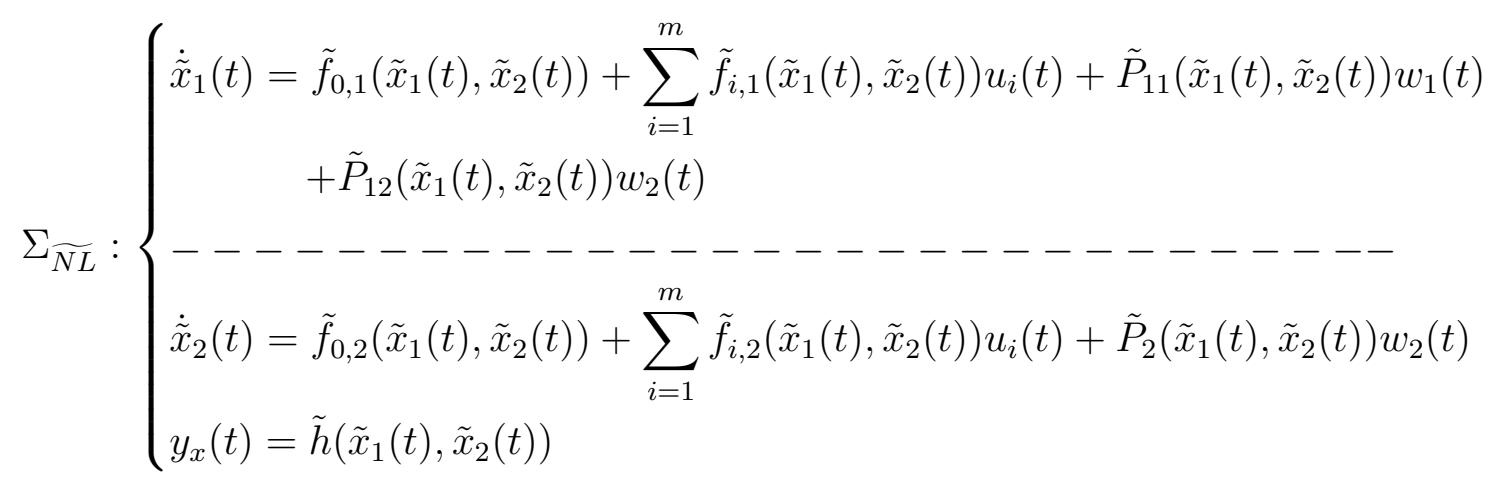

où $w_{1}(t)$ est la partie du défaut $w(t)$ à découpler et $w_{2}(t)$ l'autre partie de $w(t)$.

L'écriture (3.3) n'est en rien le reflet d'un découplage mais plutôt le résultat d'un changement de base. En effet, si un défaut apparaît $\left(w_{1}(t)\right.$ en l'occurrence) au niveau du sous-espace d'état associé à $\tilde{x}_{1}(t)$, il semble évident que ce dernier aura un effet sur l'espace d'état $\tilde{x}_{2}(t)$. Ceci s'explique par la présence de l'état $\tilde{x}_{1}(t)$ porteur du défaut dans l'écriture de l'équation différentielle régissant $\tilde{x}_{2}(t)$. Par conséquent, l'état $\tilde{x}_{2}(t)$ sera également entaché de l'effet du défaut $w_{1}(t)$.

L'utilisation d'une injection de sortie a pour objectif l'expression des composantes de $\tilde{x}_{1}(t)$ intervenant dans la dynamique de $\tilde{x}_{2}(t)$. Ainsi, à l'écriture du système (3.3) nous associons le filtre suivant :

$$
\Sigma_{\widetilde{F N L}}:\left\{\begin{array}{l}
\dot{\tilde{z}}_{1}(t)=\tilde{f}_{0,1}\left(\tilde{z}_{1}(t), \tilde{z}_{2}(t)\right)+\sum_{i=1}^{m} \tilde{f}_{i, 1}\left(\tilde{z}_{1}(t), \tilde{z}_{2}(t)\right) u_{i}(t)+\tilde{\Psi}_{1}\left(\bullet_{x}(t), \bullet_{z}(t), \bullet \bullet_{u}(t)\right) \\
-------------------------------\tilde{\Psi}_{2}\left(\bullet_{x}(t), \bullet_{z}(t), \bullet_{u}(t)\right) \\
\dot{\tilde{z}}_{2}(t)=\tilde{f}_{0,2}\left(\tilde{z}_{1}(t), \tilde{z}_{2}(t)\right)+\sum_{i=1}^{m} \tilde{f}_{i, 2}\left(\tilde{z}_{1}(t), \tilde{z}_{2}(t)\right) u_{i}(t)+\tilde{\Psi}_{z}(t)=\tilde{h}_{(}\left(\tilde{z}_{1}(t), \tilde{z}_{2}(t)\right)
\end{array}\right.
$$

où $\tilde{\Psi}\left(\bullet_{x}(t), \bullet_{z}(t), \bullet_{u}(t)\right)$ représente l'injection de sortie dont l'expression est introduite en $(2.2)$.

Nous cherchons une injection de sortie qui satisfait l'égalité suivante :

$$
\tilde{f}_{0,2}\left(\tilde{z}_{1}(t), \tilde{z}_{2}(t)\right)+\sum_{i=1}^{m} \tilde{f}_{i, 2}\left(\tilde{z}_{1}(t), \tilde{z}_{2}(t)\right) u_{i}(t)+\tilde{\Psi}_{2}\left(\bullet_{x}(t), \bullet_{z}(t), \bullet \bullet_{u}(t)\right)=\dot{\tilde{x}}_{2}(t), \forall(w(t), u(t), t)
$$

L'expression de l'injection de sortie précédemment définie est générale mais prend différentes formes en fonction des méthodes de découplage employées. L'étape de synthèse du filtre et, a fortiori, la détermination de l'injection de sortie sont la suite logique du découplage. Cependant cette synthèse n'est que trop rarement développée dans la littérature. 


\subsection{Calcul de l'injection de sortie pour le découplage}

Dans un cadre général, l'injection de sortie s'écrit $\tilde{\Psi}\left(\tilde{z}_{2}(t), \Delta\right)$ où $\Delta$ représente l'ensemble des signaux connus. En revanche, dans le cadre de l'étude menée par C. De Persi et A. Isidori, les signaux utilisés pour synthétiser l'injection de sortie sont réduits. Dans un premier temps, nous développons le calcul (i.e. l'expression explicite) de l'injection de sortie dans ce cas plus restrictif.

\subsubsection{Calcul de l'injection de sortie dans un cadre particulier}

Selon l'écriture du filtre (3.4), l'étude que nous proposons est une restriction du cas général avec :

$$
\begin{aligned}
& \tilde{\Psi}_{1}\left(\bullet_{x}(t), \bullet_{z}(t), \bullet \bullet_{u}(t)\right)=0 \\
& \text { et } \\
& \tilde{\Psi}_{2}\left(\bullet_{x}(t), \bullet_{z}(t), \bullet \bullet_{u}(t)\right)=\tilde{\Psi}\left(\tilde{z}_{2}(t), u(t), y_{x}(t)\right)-\tilde{\Psi}\left(\tilde{z}_{2}(t), u(t), y_{z}(t)\right)
\end{aligned}
$$

Nous rappelons que l'objet de ce chapitre est la synthèse d'un ou plusieurs filtres visant à l'isolation des défauts. L'étape de découplage réalisée, une détermination explicite de l'injection de sortie en accord avec l'étude de découplage finalise la synthèse du filtre. Nous distinguons les méthodes où chaque filtre est dédié à l'isolation d'un seul défaut des méthodes où un seul filtre est synthétisé dans l'objectif d'isoler tous les défauts. Dans ce paragraphe, nous supposons que les conditions d'isolation définies par les équations (2.69) et (2.70) sont satisfaites. Ainsi, il est possible d'isoler tous les défauts et de synthétiser un résidu résolvant le problème fondamental de la génération de résidus ( $c f$. équation (1.46)). Dans un premier temps, nous cherchons à synthétiser $q$ filtres, chacun d'entre eux visant l'isolation d'un seul défaut. A cette fin, il est nécessaire de déterminer $q$ sous-espaces d'état observables et sensibles à un défaut particulier et également insensibles à tous les autres défauts.

\subsubsection{Calcul de l'injection de sortie dans le cas multi-filtres}

Puisqu'il est possible d'isoler tous les défauts, d'après le calcul des différentes séquences non-décroissantes $S_{*}^{\overline{P_{j}}}$ avec $j=\{1, \cdots, q\}$, nous définissons $q$ difféomorphismes suivant la relation :

$$
\tilde{x}^{j}(t)=\Phi^{j}(x(t))=\left(\begin{array}{c}
\Phi_{1}^{j}(x(t)) \\
--- \\
\Phi_{2}^{j}(x(t))
\end{array}\right)=\left(\begin{array}{c}
\tilde{x}_{1}^{j}(t) \\
--- \\
\tilde{x}_{2}^{j}(t)
\end{array}\right) \text { avec } \frac{\partial \Phi^{j}(x(t))}{\partial x(t)}=\left(\begin{array}{c}
\left(S_{*}^{\overline{P_{j}}}\right)^{T} \\
--- \\
\left(\left(S_{*}^{\overline{P_{j}}}\right)^{\perp}\right)^{T}
\end{array}\right)
$$


Chacun de ces difféomorphismes permet la définition d'un filtre particulier :

$$
\Sigma_{\widetilde{F N L^{j}}}:\left\{\begin{aligned}
\dot{z}_{1}^{j}(t) & =\tilde{f}_{0,1}^{j}\left(\tilde{z}_{1}^{j}(t), \tilde{z}_{2}^{j}(t)\right)+\sum_{i=1}^{m} \tilde{f}_{i, 1}^{j}\left(\tilde{z}_{1}^{j}(t), \tilde{z}_{2}^{j}(t)\right) u_{i}(t) \\
\dot{\tilde{z}}_{2}^{j}(t) & =\tilde{f}_{0,2}^{j}\left(\tilde{z}_{1}^{j}(t), \tilde{z}_{2}^{j}(t)\right)+\sum_{i=1}^{m} \tilde{f}_{i, 2}^{j}\left(\tilde{z}_{1}^{j}(t), \tilde{z}_{2}^{j}(t)\right) u_{i}(t) \\
& +\tilde{\Psi}^{j}\left(\tilde{z}_{2}^{j}(t), u(t), y_{x}(t)\right)-\tilde{\Psi}^{j}\left(\tilde{z}_{2}^{j}(t), u(t), y_{z}(t)\right) \\
y_{z}(t) & =\tilde{h}^{j}\left(\tilde{z}_{1}^{j}(t), \tilde{z}_{2}^{j}(t)\right)
\end{aligned}\right.
$$

où le sous-espace d'état associé à $\tilde{z}_{1}^{j}(t)$ est le plus petit sous-espace d'état sensible au vecteur de défauts $\left(w_{1}(t) \cdots w_{j-1}(t) w_{j+1}(t) \cdots w_{q}(t)\right)^{T}$ (via l'injection de sortie (3.6)). Au contraire, le sous-espace d'état associé à $\tilde{z}_{2}^{j}(t)$ est le plus grand sous-espace d'état insensible au défaut $w_{j}(t)$. Ainsi, si l'injection de sortie $\tilde{\Psi}^{j}\left(\bullet_{x}(t), \bullet_{z}(t), \bullet_{u}(t)\right)$ est définie de manière à satisfaire l'égalité suivante :

$$
\dot{\tilde{z}}_{2}^{j}(t)=\dot{\tilde{x}}_{2}(t), \forall w_{i}(t) \text { avec } i=\{1, \cdots, j-1, j+1, \cdots, q\}
$$

il en résulte l'expression suivante de l'injection de sortie :

$$
\begin{gathered}
\tilde{f}_{0,2}^{j}\left(\tilde{z}^{j}(t)\right)+\sum_{i=1}^{m} \tilde{f}_{i, 2}^{j}\left(\tilde{z}^{j}(t)\right) u_{i}(t)=\breve{f}_{0,2}^{j}\left(\tilde{z}_{2}^{j}(t)\right)+\sum_{i=1}^{m} \breve{f}_{i, 2}^{j}\left(\tilde{z}_{2}^{j}(t)\right) u_{i}(t)+\tilde{\Psi}^{j}\left(\tilde{z}_{2}^{j}(t), u(t), y_{z}(t)\right)(t) \\
\Downarrow \\
\tilde{\Psi}^{j}\left(\tilde{z}_{2}^{j}(t), u(t), y_{z}(t)\right)(t)=\tilde{f}_{0,2}^{j}\left(\tilde{z}^{j}(t)\right)+\sum_{i=1}^{m} \tilde{f}_{i, 2}^{j}\left(\tilde{z}^{j}(t)\right) u_{i}-\left(\breve{f}_{0,2}^{j}\left(\tilde{z}_{2}^{j}(t)\right)+\sum_{i=1}^{m} \breve{f}_{i, 2}^{j}\left(\tilde{z}_{2}^{j}(t)\right) u_{i}(t)\right) \\
\text { avec } j=\{1, \cdots, q\}
\end{gathered}
$$

Tous les filtres ont donc la représentation d'état décrite par l'équation (3.11) :

$$
\Sigma_{\widetilde{F N L^{j}}}:\left\{\begin{array}{l}
\dot{z}_{1}^{j}(t)=\tilde{f}_{0,1}^{j}\left(\tilde{z}_{1}^{j}(t), \tilde{z}_{2}^{j}(t)\right)+\sum_{i=1}^{m} \tilde{f}_{i, 1}^{j}\left(\tilde{z}_{1}^{j}(t), \tilde{z}_{2}^{j}(t)\right) u_{i}(t) \\
\dot{z}_{2}^{j}(t)=\breve{f}_{0,2}^{j}\left(\tilde{z}_{2}^{j}(t)\right)+\sum_{i=1}^{m} \breve{f}_{i, 2}^{j}\left(\tilde{z}_{2}^{j}(t)\right) u_{i}(t)+\tilde{\Psi}^{j}\left(\tilde{z}_{2}^{j}(t), u(t), y_{x}(t)\right) \\
y_{z}(t)=\tilde{h}^{j}\left(\tilde{z}_{1}^{j}(t), \tilde{z}_{2}^{j}(t)\right)
\end{array}\right.
$$

Par la suite, sous respect des conditions (3.10), nous savons que l'injection de sortie satisfait la propriété d'invariance car toute distribution $\tau(x(t)) \subset \operatorname{span}\left\{d \tilde{z}_{1}^{T}\right\}$ est invariante à la dynamique du système ( $c f$. annexe $\mathrm{B}$ ). Ceci peut se traduire mathématiquement comme suit :

$$
\left[\left(\begin{array}{c}
\tilde{f}_{i, 1}^{j} \\
\breve{f}_{i, 2}^{j}
\end{array}\right), \tau(x(t))\right] \subseteq \operatorname{span}\left\{d \tilde{z}_{1}^{T}\right\}, \forall i \in\{0, \cdots, m\}
$$

Afin d'illustrer cette classe d'injection de sortie, nous reprenons l'exemple traité paragraphe 2.3.5.1. 


\section{Exemple académique}

Nous rappelons le modèle du système étudié (équation (2.57)) :

$$
\Sigma_{N L}:\left\{\begin{array}{l}
\dot{x}_{1}(t)=x_{2}(t) x_{3}(t)+w(t) \\
\dot{x}_{2}(t)=-x_{1}(t) x_{2}(t)+x_{3}^{2}(t) u(t) \\
\dot{x}_{3}(t)=x_{2}(t)-x_{3}(t) \\
y_{x 1}(t)=x_{2}(t) \\
y_{x 2}(t)=x_{3}(t)
\end{array}\right.
$$

Puisque le défaut est scalaire, nous ne synthétisons qu'un seul filtre.

Suite à l'étude menant au découplage, nous montrons qu'il est possible via une injection de sortie de rendre insensible au défaut $w(t)$ le sous-espace d'état associé à $x_{3}(t)$.

Nous cherchons donc à synthétiser un filtre de la forme (3.8) :

$$
\Sigma_{F N L}:\left\{\begin{array}{l}
\dot{z}_{1}(t)=z_{2}(t) z_{3}(t)+\Psi_{1}\left(z(t), y_{x}(t)\right)-\Psi_{1}\left(z(t), y_{z}(t)\right) \\
\dot{z}_{2}(t)=-z_{1}(t) z_{2}(t)+z_{3}^{2}(t) u(t)+\Psi_{2}\left(z(t), y_{x}(t)\right)-\Psi_{2}\left(z(t), y_{z}(t)\right) \\
\dot{z}_{3}(t)=z_{2}(t)-z_{3}(t)+\Psi_{3}\left(z(t), y_{x}(t)\right)-\Psi_{3}\left(z(t), y_{z}(t)\right) \\
y_{z 1}(t)=z_{2}(t) \\
y_{z 2}(t)=z_{3}(t)
\end{array}\right.
$$

En rappelant que la matrice jacobienne du difféomorphisme est une matrice identité définie lors de l'étude de découplage (2.63), le système transformé s'écrit:

$$
\Sigma_{\widetilde{F N L}}:\left\{\begin{array}{l}
\dot{\tilde{z}}_{1}(t)=\left(\begin{array}{l}
\dot{z}_{1}(t) \\
\dot{z}_{2}(t)
\end{array}\right)=\left(\begin{array}{c}
z_{2}(t) z_{3}(t) \\
-z_{1}(t) z_{2}(t)+z_{3}^{2}(t) u(t)
\end{array}\right) \\
\dot{\tilde{z}}_{2}(t)=\dot{z}_{3}(t)=z_{2}(t)-z_{3}(t)+\tilde{\Psi}\left(\tilde{z}(t), y_{x}(t)\right)-\tilde{\Psi}\left(\tilde{z}(t), y_{z}(t)\right) \\
y_{z 1}(t)=z_{2}(t) \\
y_{z 2}(t)=z_{3}(t)
\end{array}\right.
$$

Nous remarquons la restriction de l'injection de sortie à la seule équation différentielle régissant $\tilde{z}_{2}(t)$ ( $c f$. équation du système général (3.8)), car le sous-espace d'état associé à $\tilde{z}_{1}(t)$ est sensible au défaut.

L'objectif est d'exprimer au sein de $\dot{\tilde{z}}_{2}(t)$ tout état sensible au défaut à l'aide de l'injection de sortie. Puisque $z_{2}(t)$ est sensible au défaut nous écrivons $\tilde{\Psi}\left(\tilde{z}(t), y_{z}(t)\right)=z_{2}(t)=y_{z 1}(t)$ 
d'où le filtre suivant :

$$
\Sigma_{\widetilde{F N L}}:\left\{\begin{array}{l}
\dot{\tilde{z}}_{1}(t)=\left(\begin{array}{c}
z_{2}(t) z_{3}(t) \\
-z_{1}(t) z_{2}(t)+z_{3}^{2}(t) u(t)
\end{array}\right) \\
\dot{\tilde{z}}_{2}(t)=-z_{3}(t)+\tilde{\Psi}\left(\tilde{z}(t), y_{x}(t)\right) \\
y_{z 1}(t)=z_{2}(t) \\
y_{z 2}(t)=z_{3}(t)
\end{array}\right.
$$

avec $\tilde{\Psi}\left(\tilde{z}(t), y_{x}(t)\right)=x_{2}(t)=y_{x 1}(t)$.

Suite à cette définition, nous assurons l'égalité entre $x_{3}(t)$ et $z_{3}(t)$. Cependant, ceci n'est pas suffisant pour le diagnostic du système. En effet, une dernière étape de génération de résidu est nécessaire.

D'après les conditions exposées en (2.69) et (2.70), il est possible de déterminer au moins un résidu $r_{j}(t) y$-reconstructible, c'est-à-dire qu'il existe $r_{j}(t)$ tel que :

$$
\begin{aligned}
r_{j}(t) & =\chi^{\overline{P_{j}}} \circ h(x(t))-\chi^{\overline{P_{j}}} \circ h(z(t)) \\
& =\chi^{\overline{P_{j}}} \circ h\left(\tilde{x}_{2}(t)\right)-\chi^{\overline{P_{j}}} \circ h\left(\tilde{z}_{2}(t)\right)
\end{aligned}
$$

La synthèse de $q$ résidus de la forme (3.17), (chaque résidu n'étant sensible qu'au seul défaut $w_{j}(t)$ ), permet l'isolation de toutes les composantes du défaut $w(t)$. Cependant la définition des $q$ filtres peut, dans un cadre industriel, devenir une source de problèmes liés par exemple à la charge de calculs ou à l'espace mémoire requis. C'est pourquoi, dans la section suivante nous proposons la synthèse d'un seul filtre dédié à la localisation de chacune des composantes du vecteur défaut $w(t)$.

\subsubsection{Calcul de l'injection de sortie dans le cas mono-filtre}

La synthèse d'un unique filtre isolateur de défauts, nécessite des hypothèses de construction plus contraignante ([40]). En effet, pour isoler le vecteur défaut à l'aide d'un seul filtre, il est nécessaire de satisfaire à de nouvelles caractéristiques géométriques. Ainsi, nous décomposons l'ensemble des distributions $S_{*}^{P_{j}}$ (avec $j=\{1, \cdots, q\}$ ) en sommes de distributions mettant en relief leurs intersections. Nous recherchons au sein du sous-espace d'état $S_{*}^{P_{j}}$ les distributions sensibles à d'autres défauts. Ainsi $\phi^{P_{i}, \cdots, P_{j}}$ est défini comme étant la partie commune au sens strict des distributions $S_{*}^{P_{i}}, \cdots$, et $S_{*}^{P_{j}}$.

La distribution $\phi^{P_{i}, \cdots, P_{j}}$ vérifie les propriétés suivantes :

(i) $\phi^{P_{i}, \cdots, P_{j}} \subseteq S_{*}^{P_{i}} \cap \cdots \cap S_{*}^{P_{j}} \neq\{0\}$ avec $i \neq j$,

(ii) $\phi^{P_{i}, \cdots, P_{j}} \cap S_{*}^{P_{k}}=\{0\}$ avec $k \neq\{i, \cdots, j\}$, 
Remarquons que $\phi^{P_{i}, \cdots, P_{j}}$ n'est défini que dans le cas de non réduction à la distribution nulle $(\{0\})$. Par définition $\phi^{P_{j}}$ est une distribution du sous-espace d'état $S_{*}^{P_{j}}$ ne possédant aucune intersection avec $S_{*}^{P_{k}}$ où $k=\{1, \cdots, j-1, j+1, \cdots, q\}$. Suite à ces définitions, nous récrivons le sous-espace d'état $S_{*}^{P_{j}}$ :

$$
S_{*}^{P_{j}}=\phi^{P_{j}} \oplus \phi^{P_{j}, P_{i}} \oplus \cdots \oplus \phi^{\cdots, P_{j}, \cdots}
$$

La non réduction à $\{0\}$ de la distribution $\phi^{P_{j}}$ est particulièrement importante à prouver pour la synthèse du filtre et du résidu.

Théorème 3.1 Si tous les défauts constituant le vecteur défaut $w(t)$ sont isolables d'après les conditions de C. De Persis et A. Isidori ((2.69) et (2.70)) alors à chaque défaut $w_{j}(t)$, $j=\{1, \cdots, q\}$, il est possible d'associer une distribution notée $\phi^{P_{j}}$ uniquement sensible au défaut $w_{j}(t)$.

\section{Preuve}

Cette démonstration s'appuie sur les conditions d'isolation décrites en (2.69) et (2.70). En effet, ces dernières assurent l'inclusion $\operatorname{span}\left\{P_{j}\right\} \subseteq\left(S_{*}^{\overline{P_{j}}}\right)^{\perp}$ et par définition de la séquence non décroissante $S_{*}^{P_{j}}$ nous avons alors $\operatorname{span}\left\{P_{j}\right\} \subseteq S_{*}^{P_{j}}$.

De plus $S_{*}^{P_{i}} \subseteq S_{*}^{\overline{P_{j}}}$ avec $i=\{1, \cdots, j-1, j+1, \cdots, q\}$. Nous précisons également que d'après la définition de l'involutivité d'une distribution (cf. annexe A définition A.1) $\left(\bigcup_{i=1, i \neq j}^{q} S_{*}^{P_{i}}\right) \subseteq$ $S_{*}^{\overline{P_{j}}}$. Ceci implique la suite d'inclusions suivante $: \operatorname{span}\left\{P_{j}\right\} \subseteq\left(S_{*}^{\overline{P_{j}}}\right)^{\perp} \subseteq\left(\bigcup_{i=1, i \neq j}^{q} S_{*}^{P_{i}}\right)^{\perp}$. Pour conclure cette démonstration, il est facile de comprendre que $\phi^{P_{j}} \neq\{0\}$ car cette distribution est au minimum réduite à $\operatorname{span}\left\{P_{j}\right\} \neq\{0\}$.

Il est plus aisé d'appréhender ces différentes notions et inclusions à l'aide d'un schéma tel que celui représenté à la figure 3.1 où le cas de quatre défauts est considéré. Afin de simplifier la représentation, nous ne faisons pas figurer sur le schéma l'élément $\{0\}$ commun à chaque sousespace. Ce schéma établit un sens graphique aux différentes inclusions telles que $: \operatorname{span}\left\{P_{1}\right\} \subseteq$ $S_{*}^{P_{1}}$ ou encore la triple inclusion de $S_{*}^{P_{2}}, S_{*}^{P_{3}}$ et $S_{*}^{P_{4}}$ dans $S_{*}^{\overline{P_{1}}}$.

Par construction, nous savons que $S_{*}^{P_{j}}$ est au minimum (au sens des inclusions) égal à $\operatorname{span}\left\{P_{j}\right\}$. Or d'après le théorème $3.1 \operatorname{span}\left\{P_{j}\right\}$ est aussi une propriété de $\phi^{P_{j}}$.

Ainsi les $q$ premiers éléments qui définissent le difféomorphisme $(\tilde{x}(t)=\Phi(x(t)))$ sont non nuls :

$$
\frac{\partial \Phi(x(t))}{\partial x(t)}=\left(\begin{array}{lllllll}
\phi^{P_{1}} & \cdots & \phi^{P_{q}} & \phi^{P_{k 1}, P_{k 2}} & \cdots & \phi^{P_{k 3}, \cdots, P_{k 4}} & \gamma
\end{array}\right)^{T}
$$




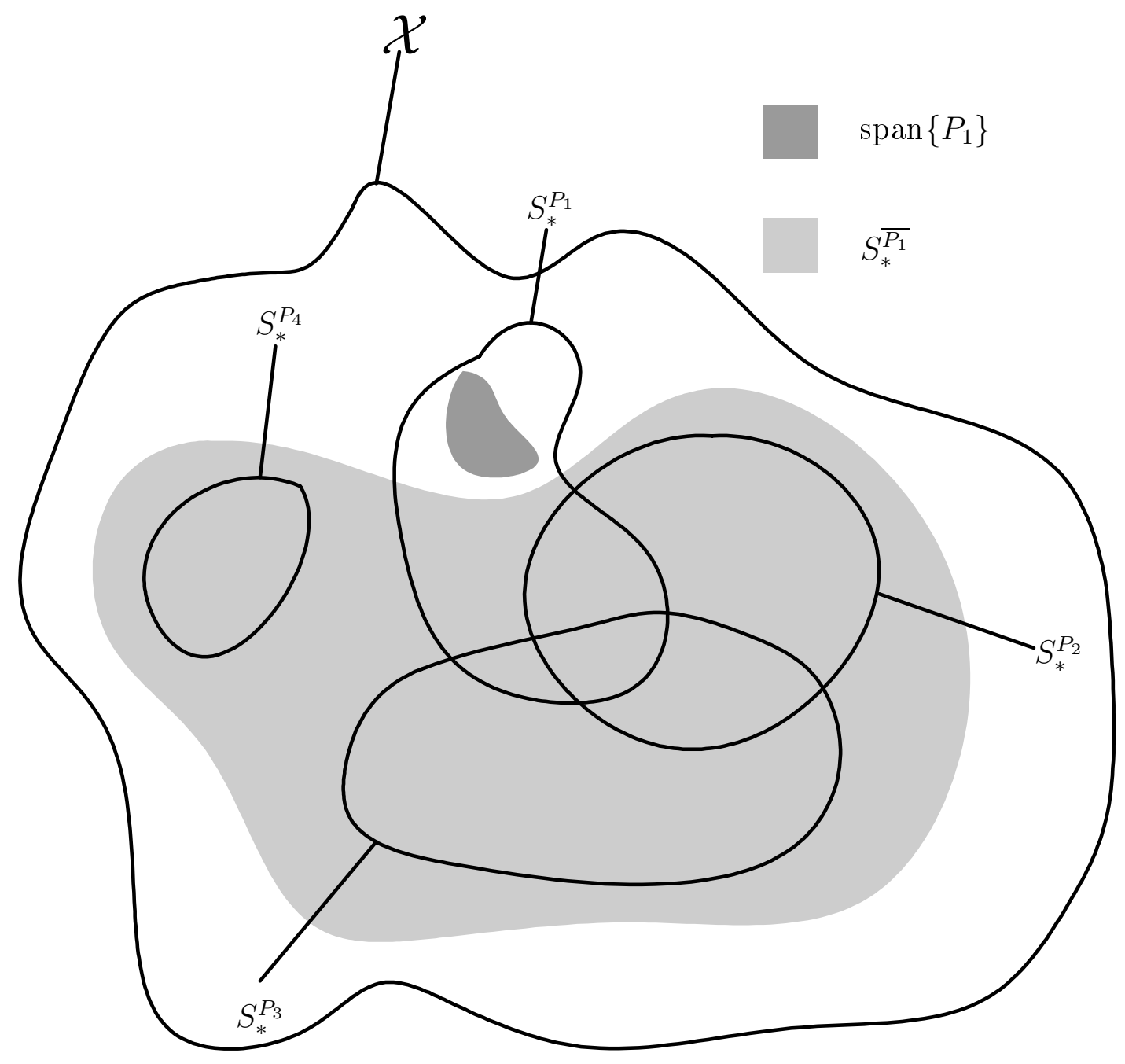

FIG. 3.1: Schématisation de l'espace d'état et de ses sous-espaces 
où la distribution $\gamma$ est choisie de manière à assurer l'intégration de $\frac{\partial \Phi(x(t))}{\partial x(t)}$ et est orthogonale aux autres distributions $\left(\right.$ i.e. $\left.\gamma \subset\left(\phi^{\bullet}\right)^{\perp}, \forall \bullet\right)$. Chaque distribution $\phi^{\bullet}$ provient de la décomposition des distributions $S_{*}^{P_{j}}$ avec $j=\{1, \cdots, q\}$ donc $k 1, k 2, k 3, \cdots, k 4 \in\{1, \cdots, q\}$. Posons $\tilde{x}(t)=\left(\begin{array}{lllllll}\tilde{x}_{1}(t) & \cdots & \tilde{x}_{q}(t) & \tilde{x}_{k 1, k 2}(t) & \cdots & \tilde{x}_{k 3, \cdots, k 4}(t) & \left.\tilde{x}_{\gamma}(t)\right)^{T}=\Phi(x(t)) .\end{array}\right.$

Dans ce nouveau système de coordonnées, afin d'assurer les découplages entre les différentes variables $\tilde{x}_{\bullet}(t)$, une hypothèse supplémentaire de construction doit être satisfaite.

Si nous prenons l'exemple des deux distributions $\phi^{P_{i}}$ et $\phi^{P_{i}, P_{j}}$, nous cherchons à l'aide d'une injection de sortie à rendre l'état $\tilde{x}_{i}(t)$ exclusivement sensible au défaut $w_{i}(t)$. Cependant ceci n'est possible que si la distribution $\phi^{P_{i}}$ est orthogonale à toutes les autres distributions $\phi^{\cdots, P_{k}, \cdots}$ avec $k \neq i$.

De la même façon, pour que l'état $\tilde{x}_{i, j}(t)$ ne soit sensible qu'aux défauts $w_{i}(t)$ et $w_{j}(t)$, la distribution $\phi^{P_{i}, P_{j}}$ doit être orthogonale à toutes les distributions $\phi^{\cdots, P_{k}, \cdots}$ avec $k \neq i, j$.

En suivant le même raisonnement avec l'ensemble des distributions nous supposons les distributions décrivant les sous-espaces d'état $S_{*}^{P_{j}}$ orthogonales entre elles (avec $j=\{1, \cdots, q\}$ ).

Pour résumer, l'hypothèse dite de construction ou de décomposition en somme directe est la suivante avec $j=\{1, \cdots, q\}$ :

$$
S_{*}^{P_{j}}=\phi^{P_{j}} \oplus \phi^{P_{j}, P_{i}} \oplus \cdots \oplus \phi^{\cdots, P_{j}, \cdots}
$$

où l'ensemble des distributions $\phi^{\bullet}$ sont orthogonales entre elles.

Enfin pour terminer nous assurons que $\operatorname{span}\left\{d x_{\gamma}^{T}\right\}$ est insensible au vecteur défaut $w(t)$ via une injection de sortie.

Sous cette hypothèse, une détermination explicite de l'injection de sortie assurant l'invariance des sous-espaces d'état est possible.

Afin de mieux appréhender les problèmes liés à la non satisfaction de l'hypothèse d'orthogonalité des éléments qui composent la somme (3.20), nous proposons une étude de cas.

\section{Etude de cas}

Dans un premier temps, nous étudions un cas où l'hypothèse proposée n'est pas satisfaite et les difficultés résultantes sont mises en relief. Dans un deuxième temps, nous montrons que l'hypothèse de décomposition est satisfaite.

\section{Cas 1}


Considérons les deux distributions suivantes :

$$
S_{*}^{P_{1}}=\operatorname{span}\left\{\left(\begin{array}{l}
0 \\
0 \\
0 \\
1
\end{array}\right),\left(\begin{array}{l}
0 \\
1 \\
1 \\
0
\end{array}\right)\right\} S_{*}^{P_{2}}=\operatorname{span}\left\{\left(\begin{array}{l}
1 \\
0 \\
0 \\
0
\end{array}\right),\left(\begin{array}{l}
0 \\
1 \\
0 \\
0
\end{array}\right)\right\}
$$

alors $\phi^{P_{1}}=S_{*}^{P_{1}}, \phi^{P_{2}}=S_{*}^{P_{2}}$ et $\phi^{P_{1}, P_{2}}$ n'est pas définie car $S_{*}^{P_{1}} \cap S_{*}^{P_{2}}=\{0\}$. Ainsi l'équation (3.20) se réduit à :

$$
S_{*}^{P_{1}}=\phi^{P_{1}} \text { et } S_{*}^{P_{2}}=\phi^{P_{2}}
$$

Cependant $\phi^{P_{1}}$ n'est pas orthogonale à $\phi^{P_{2}}$ donc l'hypothèse de construction n'est pas satisfaite. Ainsi nous ne pouvons pas assurer le découplage entre ces deux sous-espaces d'état. Si nous suivons, tout de même, la méthodologie précédemment décrite avec une autre définition de distributions orthogonales $\phi^{P_{1}}$ et $\phi^{P_{2}}$ (cf. équation (3.23)), le difféomorphisme $\Phi(x(t))$ est défini par :

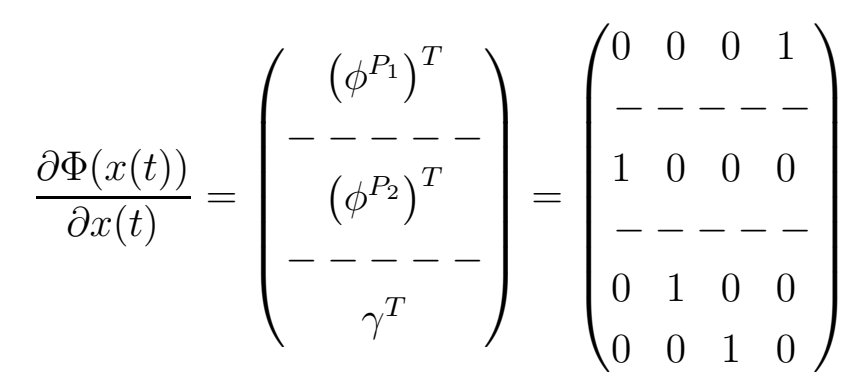

avec $\gamma \subseteq\left(\phi^{P_{1}}\right)^{\perp}$ et $\gamma \subseteq\left(\phi^{P_{2}}\right)^{\perp}$.

Or ce difféomorphisme ne résulte pas d'une décomposition en somme directe donc les sensibilités des distributions ne peuvent être déduites. En effet, $\gamma$ n'est pas insensible au vecteur défaut $w(t)$ car la distribution (élément générateur de $\gamma$ ) $\left(\begin{array}{llll}0 & 1 & 0 & 0\end{array}\right)^{T}$ est incluse ni dans $\left(S_{*}^{P_{1}}\right)^{\perp}$ ni dans $\left(S_{*}^{P_{2}}\right)^{\perp}$. Elle est donc sensible aux défauts $w_{1}(t)$ et $w_{2}(t)$.

Ainsi, il n'est pas possible de garantir que la propagation du défaut $w_{2}(t)$ sera restreinte au sous-espace d'état $S_{*}^{P_{2}}$. Ce raisonnement peut être reproduit pour la distribution $\left(\begin{array}{llll}0 & 0 & 1 & 0\end{array}\right)^{T}$ . C'est-à-dire qu'il n'est pas possible d'empêcher via une injection de sortie la propagation de l'effet des défauts $w_{1}(t)$ et $w_{2}(t)$ au travers de l'espace d'état au moyen d'un unique filtre.

\section{Cas 2}

Nous considérons les deux distributions suivantes :

$$
S_{*}^{P_{1}}=\operatorname{span}\left\{\left(\begin{array}{l}
0 \\
0 \\
0 \\
1
\end{array}\right),\left(\begin{array}{l}
0 \\
1 \\
0 \\
0
\end{array}\right)\right\} S_{*}^{P_{2}}=\operatorname{span}\left\{\left(\begin{array}{l}
1 \\
0 \\
0 \\
0
\end{array}\right),\left(\begin{array}{l}
0 \\
1 \\
0 \\
0
\end{array}\right)\right\}
$$


$\phi^{P_{1}}$ et $\phi^{P_{2}}$ sont identiques au cas précédent, en revanche $\phi^{P_{1}, P_{2}}=\operatorname{span}\left\{\left(\begin{array}{llll}0 & 1 & 0 & 0\end{array}\right)^{T}\right\}$.

De plus, l'hypothèse de décomposition en somme directe est satisfaite :

$$
\begin{aligned}
& S_{*}^{P_{1}}=\phi^{P_{1}} \oplus \phi^{P_{1}, P_{2}} \text { avec } \phi^{P_{1}} \subseteq\left(\phi^{P_{2}}\right)^{\perp} \text { et } \phi^{P_{1}} \subseteq\left(\phi^{P_{1}, P_{2}}\right)^{\perp} \\
& S_{*}^{P_{2}}=\phi^{P_{2}} \oplus \phi^{P_{1}, P_{2}} \text { avec } \phi^{P_{2}} \subseteq\left(\phi^{P_{1}}\right)^{\perp} \text { et } \phi^{P_{2}} \subseteq\left(\phi^{P_{1}, P_{2}}\right)^{\perp}
\end{aligned}
$$

Le difféomorphisme décrit en (3.19) s'écrit :

$$
\frac{\partial \Phi(x(t))}{\partial x(t)}=\left(\begin{array}{c}
\left(\phi^{P_{1}}\right)^{T} \\
----- \\
\left(\phi^{P_{2}}\right)^{T} \\
----- \\
\left(\phi^{P_{1}, P_{2}}\right)^{T} \\
----- \\
(\gamma)^{T}
\end{array}\right)=\left(\begin{array}{cccc}
0 & 0 & 0 & 1 \\
- & - & - & - \\
1 & 0 & 0 & 0 \\
- & - & - & - \\
0 & 1 & 0 & 0 \\
- & - & - & - \\
0 & 0 & 1 & 0
\end{array}\right)
$$

Nous assurons alors la possibilité d'obtenir via une injection de sortie les caractéristiques désirées en terme d'invariance et de sensibilité.

L'hypothèse de décomposition en somme directe (3.20) satisfaite, le système général (3.1) suite à l'application de la transformation (3.19) s'écrit comme suit :

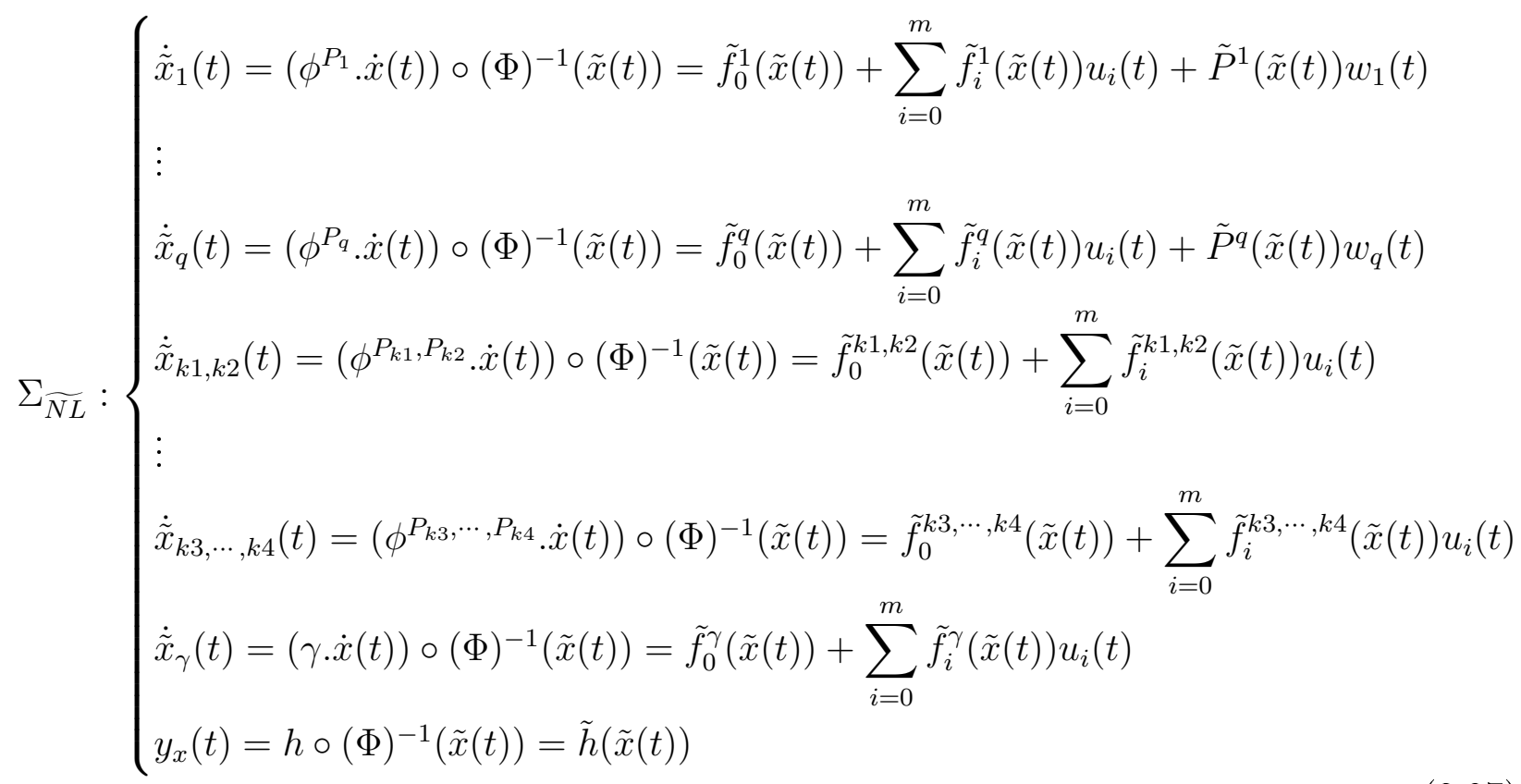

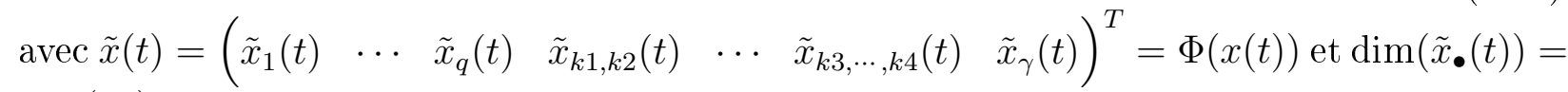
$\operatorname{dim}\left(\phi^{\bullet}\right)$.

Notons que $w_{j}(t)$ apparaît seulement au niveau de l'équation différentielle régissant la $j^{\mathrm{e}}$ composante d'état $\tilde{x}_{j}(t)$ avec $j=\{1, \cdots, q\}$. Cette remarque est évidemment la conséquence du 
théorème 3.1 .

A partir de cette écriture, nous développons la détermination de l'injection de sortie définissant le filtre suivant :

$\Sigma_{\widetilde{F N L}}:\left\{\begin{array}{l}\dot{\tilde{z}}_{1}(t)=\tilde{f}_{0}^{1}(\tilde{z}(t))+\sum_{i=0}^{m} \tilde{f}_{i}^{1}(\tilde{z}(t)) u_{i}(t)+\tilde{\Psi}^{1}\left(\tilde{z}(t), u(t), y_{x}(t)\right)-\tilde{\Psi}^{1}\left(\tilde{z}(t), u(t), y_{z}(t)\right) \\ \vdots \\ \dot{\tilde{z}}_{q}(t)=\tilde{f}_{0}^{q}(\tilde{z}(t))+\sum_{i=0}^{m} \tilde{f}_{i}^{q}(\tilde{z}(t)) u_{i}(t)+\tilde{\Psi}^{q}\left(\tilde{z}(t), u(t), y_{x}(t)\right)-\tilde{\Psi}^{q}\left(\tilde{z}(t), u(t), y_{z}(t)\right) \\ \quad+\tilde{\Psi}^{k 1, k 2}\left(\tilde{z}(t), u(t), y_{x}(t)\right)-\tilde{\Psi}^{k 1, k 2}\left(\tilde{z}(t), u(t), y_{z}(t)\right) \\ \quad+\tilde{z}_{k 1, k 2}(t)=\tilde{f}^{k 1, k 2}(\tilde{z}(t))+\sum_{i=0}^{k 1, \cdots, k 4}\left(\tilde{z}(t), u(t), y_{x}(t)\right)-\tilde{\Psi}(t) u_{i}(t) \\ \dot{\tilde{z}}_{k 3, \cdots, k 4}(t)=\tilde{f}_{0}^{k 3, \cdots, k 4}(\tilde{z}(t))+\sum_{i=0}^{m} \tilde{f}_{i}^{k 3, \cdots, k 4}(\tilde{z}(t)) u_{i}(t) \\ \left.\dot{\tilde{z}}_{\gamma}(t)=\tilde{f}_{0}^{\gamma}(\tilde{z}(t))+\sum_{i=0}^{m} \tilde{f}_{i}^{\gamma}(\tilde{z}(t)) u_{i}(t)+\tilde{\Psi}^{\gamma}\left(\tilde{z}(t), u(t), y_{x}(t)\right)-\tilde{\Psi}_{z}^{\gamma}(t)\right) \\ \left.y_{z}(t)=\tilde{z}(t), u(t), y_{z}(t)\right)\end{array}\right.$

Sous les hypothèses développées d'une part par C. De Persis et A. Isodori ((2.69) et (2.70)) et d'autre part introduite dans cette section par l'équation (3.20), il est possible de récrire le filtre comme suit :

$$
\Sigma_{\widetilde{F N L}}:\left\{\begin{array}{l}
\dot{\tilde{z}}_{1}(t)=\breve{f}_{0}^{1}\left(\breve{z}_{*}^{1}(t)\right)+\sum_{i=0}^{m} \breve{f}_{i}^{1}\left(\breve{z}_{*}^{1}(t)\right) u_{i}(t)+\tilde{\Psi}^{1}\left(\breve{z}_{*}^{1}(t), u(t), y_{x}(t)\right) \\
\vdots \\
\dot{\tilde{z}}_{q}(t)=\breve{f}_{0}^{q}\left(\breve{z}_{*}^{q}(t)\right)+\sum_{i=0}^{m} \breve{f}_{i}^{q}\left(\breve{z}_{*}^{q}(t)\right) u_{i}(t)+\tilde{\Psi}^{q}\left(\breve{z}_{*}^{q}(t), u(t), y_{x}(t)\right) \\
\dot{\tilde{z}}_{k 1, k 2}(t)=\breve{f}_{0}^{k 1, k 2}\left(\breve{z}_{*}^{k 1, k 2}(t)\right)+\sum_{i=0}^{m} \breve{f}_{i}^{k 1, k 2}\left(\breve{z}_{*}^{k 1, k 2}(t)\right) u_{i}(t)+\tilde{\Psi}^{k 1, k 2}\left(\breve{z}_{*}^{k 1, k 2}(t), u(t), y_{x}(t)\right) \\
\dot{\tilde{z}}_{k 3, \cdots, k 4}(t)=\breve{f}_{0}^{k 3, \cdots, k 4}\left(\breve{z}_{*}^{k 3, \cdots, k 4}(t)\right)+\sum_{i=0}^{m} \breve{f}_{i}^{k 3, \cdots, k 4}\left(\breve{z}_{*}^{k 3, \cdots, k 4}(t)\right) u_{i}(t) \\
\quad+\tilde{\Psi}^{k 3, \cdots, k 4}\left(\breve{z}_{*}^{k 3, \cdots, k 4}(t), u(t), y_{x}(t)\right) \\
\dot{\tilde{z}}_{\gamma}(t)=\breve{f}_{0}^{\gamma}\left(\breve{z}_{*}^{\gamma}(t)\right)+\sum_{i=0}^{m} \breve{f}_{i}^{\gamma}\left(\breve{z}_{*}^{\gamma}(t)\right) u_{i}(t)+\tilde{\Psi}^{\gamma}\left(\breve{z}_{*}^{\gamma}(t), u(t), y_{x}(t)\right) \\
y_{z}(t)=\tilde{h}(\tilde{z}(t))
\end{array}\right.
$$


avec

$$
\begin{aligned}
& \left(\begin{array}{lllllll}
\breve{z}_{*}^{1}(t) & \cdots & \breve{z}_{*}^{q}(t) & \breve{z}_{*}^{k 1, k 2}(t) & \cdots & \breve{z}^{k 3, \cdots, k 4}(t) & \breve{z}_{*}^{\gamma}(t)
\end{array}\right)= \\
& \left(\left[\tilde{z}_{1}(t), \tilde{z}_{\gamma}(t)\right] \cdots\left[\tilde{z}_{q}(t), \tilde{z}_{\gamma}(t)\right] \quad\left[\tilde{z}_{k 1}(t), \tilde{z}_{k 2}(t), \tilde{z}_{k 1, k 2}(t), \tilde{z}_{\gamma}(t)\right]\right. \\
& \left.\cdots \quad\left[\tilde{z}_{k 3}(t), \cdots, \tilde{z}_{k 4}(t), \tilde{z}_{k 3, \cdots, k 4}(t), \tilde{z}_{\gamma}(t)\right] \quad\left[\tilde{z}_{\gamma}(t)\right]\right)
\end{aligned}
$$

Ainsi chaque composante de l'injection de sortie a pour objectif l'expression des contributions des autres états ne s'inscrivant pas dans la définition (3.30). En précisant cette idée, nous donnons les égalités suivantes:

$$
\begin{gathered}
\breve{f}_{0}^{\bullet}\left(\breve{z}_{*}^{\bullet}(t)\right)+\sum_{i=0}^{m} \breve{f}_{i}^{\bullet}\left(\breve{z}_{*}^{\bullet}(t)\right) u_{i}(t)+\tilde{\Psi}^{\bullet}\left(\breve{z}_{*}^{\bullet}(t), u(t), y_{z}(t)\right)=\tilde{f}_{0}^{\bullet}(\tilde{z}(t))+\sum_{i=0}^{m} \tilde{f}_{i}^{\bullet}(\tilde{z}(t)) u_{i}(t) \\
\Downarrow \\
\tilde{\Psi}^{\bullet}\left(\breve{z}_{*}^{\bullet}(t), u(t), y_{z}(t)\right)=\tilde{f}_{0}^{\bullet}(\tilde{z}(t))+\sum_{i=0}^{m} \tilde{f}_{i}^{\bullet}(\tilde{z}(t)) u_{i}(t)-\left(\breve{f}_{0}^{\bullet}\left(\breve{z}_{*}^{\bullet}(t)\right)+\sum_{i=0}^{m} \breve{f}_{i}^{\bullet}\left(\breve{z}_{*}^{\bullet}(t)\right) u_{i}(t)\right)
\end{gathered}
$$

avec $\bullet=\{[1], \cdots,[q],[k 1, k 2], \cdots,[k 3, \cdots, k 4],[\gamma]\}$.

L'équation (3.31) détermine explicitement l'injection de sortie menant à la synthèse d'un filtre isolateur du vecteur des défauts $w(t)$.

L'hypothèse de $y$-reconstructabilité d'une partie de $\phi^{P_{j}}, j=\{1, \cdots, q\}$ géométriquement traduite par l'intersection non nulle (2.69), assure l'existence d'un résidu à $q$ composantes. Chaque composante $r_{j}(t)$ est alors sensible à un seul défaut $w_{j}(t)$ avec :

$$
\exists r_{j}(t) \text { tel que } \chi^{\overline{P_{j}}} \circ \tilde{h}(\tilde{x}(t))=\chi^{\overline{P_{j}}}\left(\breve{x}_{*}^{j}\right)
$$

où $\breve{x}_{*}^{j}$ est associé à $\breve{z}_{*}^{j}$.

L'équation du filtre définie en (3.29) constitue avec (3.32) l'ensemble des éléments permettant l'isolation des défauts. Ce filtre isolateur répond au problème fondamental de la génération de résidus (F.P.R.G.).

Cependant lors du chapitre précédent, nous avions montré qu'il était possible d'isoler l'ensemble des défauts sans qu'il soit nécessaire de répondre au problème fondamental de la génération de résidus. Dans le paragraphe suivant nous proposons une méthode menant à la détermination de l'injection de sortie suite à une analyse structurelle.

\subsubsection{Analyse structurelle pour la synthèse d'un filtre d'isolation}

Une condition de rang qui doit être satisfaite pour que l'isolation des défauts soit possible est énoncée au théorème 2.12. Cette alternative au F.P.R.G. publiée dans [41] constitue une première étape. Dans cette section, nous proposons une méthode permettant la synthèse du 
filtre correspondant.

Cette dernière s'apparente beaucoup à la précédente, mais les différences, que nous mettons en relief justifient son développement.

Ainsi l'hypothèse de départ est identique à l'équation (3.20) :

$$
S_{*}^{P_{j}}=\phi^{P_{j}} \oplus \phi^{P_{j}, P_{i}} \oplus \cdots \oplus \phi^{\cdots, P_{j}, \cdots}
$$

En revanche, le théorème 3.1 n'est plus applicable car les conditions d'isolation ne sont plus celles de C. De Persis et A. Isidori. En effet, la distribution $\phi^{P_{j}}, j=\{1, \cdots, q\}$ peut à présent être nulle.

Suite à la transformation $\tilde{x}(t)=\Phi(x(t))$, le système (3.1) s'écrit comme suit :

$$
\Sigma_{\widetilde{N L}}:\left\{\begin{array}{l}
\dot{\tilde{x}}_{k 1}(t)=\tilde{f}_{0}^{k 1}(\tilde{x}(t))+\sum_{i=0}^{m} \tilde{f}_{i}^{k 1}(\tilde{x}(t)) u_{i}(t)+\tilde{P}^{k 1}(\tilde{x}(t)) w_{k 1}(t) \\
\vdots \\
\dot{\tilde{x}}_{k 2}(t)=\tilde{f}_{0}^{k 2}(\tilde{x}(t))+\sum_{i=0}^{m} \tilde{f}_{i}^{k 2}(\tilde{x}(t)) u_{i}(t)+\tilde{P}^{k 2}(\tilde{x}(t)) w_{k 2}(t) \\
\dot{\tilde{x}}_{k 3, k 4}(t)=\tilde{f}_{0}^{k 3, k 4}(\tilde{x}(t))+\sum_{i=0}^{m} \tilde{f}_{i}^{k 3, k 4}(\tilde{x}(t)) u_{i}(t)+\sum_{l=\{k 3, k 4\}} \tilde{P}_{l}^{k 3, k 4}(\tilde{x}(t)) w_{l}(t) \\
\dot{\tilde{x}}_{k 5, \cdots, k 6}(t)=\tilde{f}_{0}^{k 5, \cdots, k 6}(\tilde{x}(t))+\sum_{i=0}^{m} \tilde{f}_{i}^{k 5, \cdots, k 6}(\tilde{x}(t)) u_{i}(t)+\sum_{l=\{k 5, \cdots, k 6\}} \tilde{P}_{l}^{k 5, \cdots, k 6}(\tilde{x}(t)) w_{l}(t) \\
\dot{\tilde{x}}_{\gamma}(t)=\tilde{f}_{0}^{\gamma}(\tilde{x}(t))+\sum_{i=0}^{m} \tilde{f}_{i}^{\gamma}(\tilde{x}(t)) u_{i}(t) \\
y_{x}(t)=(h) \circ(\Phi)^{-1}(\tilde{x}(t))=\tilde{h}(\tilde{x}(t))
\end{array}\right.
$$

avec $\tilde{x}(t)=\left(\begin{array}{lllllll}\tilde{x}_{k 1}(t) & \cdots & \tilde{x}_{k 2}(t) & \tilde{x}_{k 3, k 4}(t) & \cdots & \tilde{x}_{k 5, \cdots, k 6}(t) & \tilde{x}_{\gamma}(t)\end{array}\right)^{T}$

,$\frac{\partial \Phi(x(t))}{\partial x(t)}=\left(\begin{array}{lllllll}\phi^{P_{k 1}} & \cdots & \phi^{P_{k 2}} & \phi^{P_{k 3}, P_{k 4}} & \cdots & \phi^{P_{k 5}, \ldots, P_{k 6}} & \gamma\end{array}\right)^{T}$ et $k 1, \cdots, k 6 \in\{1, \cdots, q\}$.

Puisque span $\left\{P_{j}\right\}$ n'est plus nécessairement inclus dans $\phi^{P_{j}}$, le défaut $w_{j}(t)$ apparaît dans l'écriture de chaque composante issue de l'intersection avec $S_{*}^{P_{j}}$. Ainsi, $w_{j}(t)$ est a priori présent sur chaque état $\left(\begin{array}{llll}\tilde{x}_{j}(t) & \tilde{x}_{j, i}(t) & \cdots & \tilde{x}_{\ldots, j, \ldots}(t)\end{array}\right)$. La figure 3.2 donne un sens graphique à nos développements. Elle se différentie de la figure 3.1 et 3.2 en terme d'intersection entre $\operatorname{span}\left\{P_{1}\right\}, S_{*}^{P_{2}}$ et $S_{*}^{P_{3}}$.

En ce qui concerne la détermination explicite de l'injection de sortie, la méthode employée est identique à celle de la section précédente et n'est ainsi pas rappelée.

Nous développons à présent un exemple illustrant le calcul proposé de l'injection de sortie. 


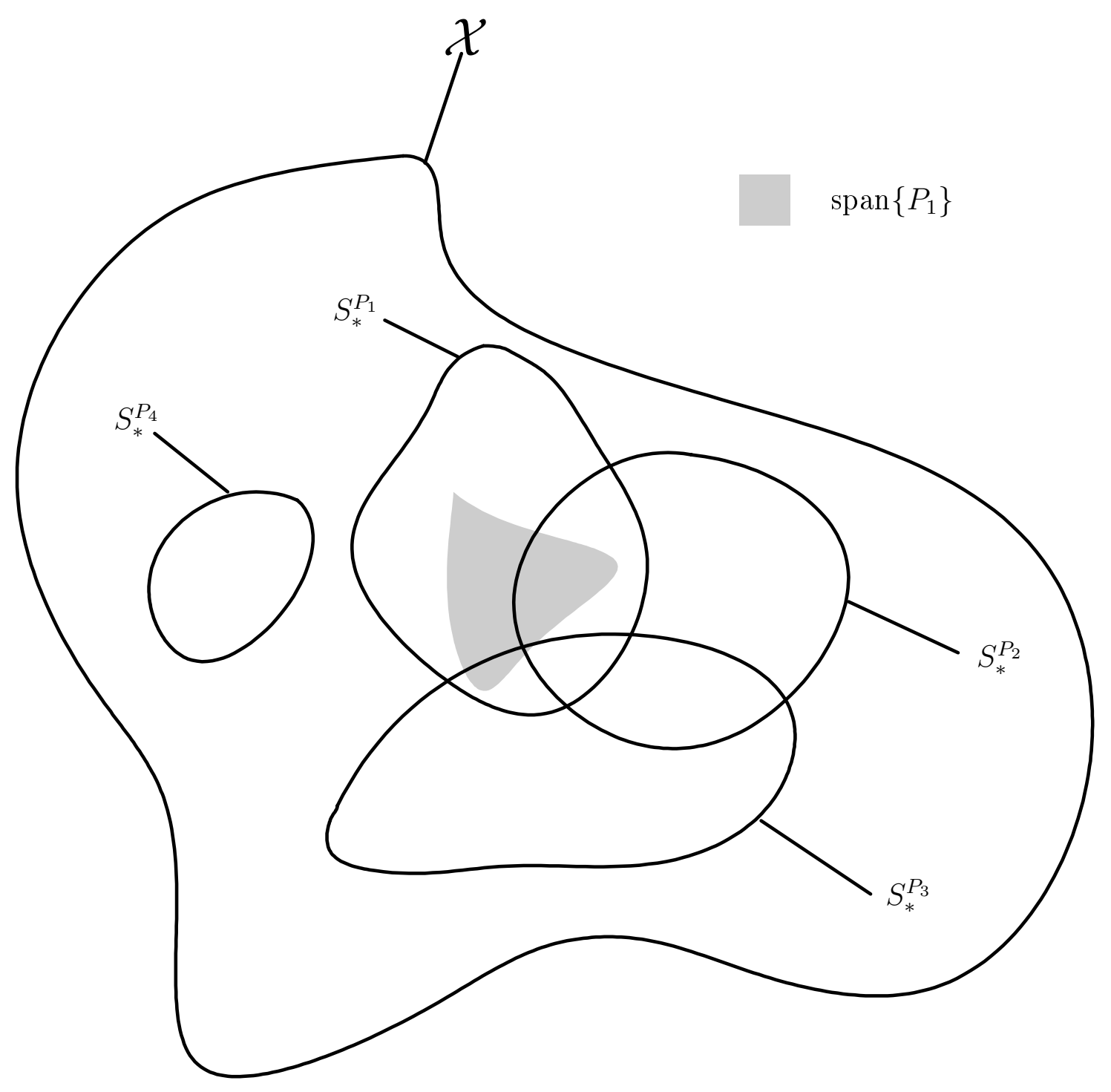

FiG. 3.2: Schématisation de l'espace d'état et de ses sous-espaces 


\section{Exemple académique}

Le système considéré est modélisé comme suit :

$$
\Sigma_{N L}:\left\{\begin{array}{l}
\dot{x}_{1}(t)=x_{1}(t)+u_{1}(t)+w_{1}(t) \\
\dot{x}_{2}(t)=x_{3}(t) x_{4}^{2}(t)+x_{1}(t) u_{2}(t)+w_{2}(t) \\
\dot{x}_{3}(t)=x_{2}(t) x_{3}(t)+x_{4}(t) \\
\dot{x}_{4}(t)=x_{4}^{2}(t)+u_{3}(t)+w_{3}(t) \\
y_{x}(t)=\left[\begin{array}{lll}
x_{1}^{2}(t) & x_{2}(t) x_{3}(t) & x_{3}(t)
\end{array}\right]^{T}
\end{array}\right.
$$

avec $x(t) \in \mathbb{R}^{4}, u(t) \in \mathbb{R}^{3}, y_{x}(t) \in \mathbb{R}^{3}$ et $w(t) \in \mathbb{R}^{3}$ qui sont respectivement les états, commandes, sorties et défauts du système non linéaire affine en la commande. D'après cette représentation, nous posons $\left(\begin{array}{lll}P_{1} & P_{2} & P_{3}\end{array}\right)^{T}=\left(\begin{array}{llll}1 & 0 & 0 & 0 \\ 0 & 1 & 0 & 0 \\ 0 & 0 & 0 & 1\end{array}\right)$. Nous supposons que les états $x_{1}(t), x_{2}(t)$ et $x_{3}(t)$ sont non nuls et donc $\operatorname{ker}\{d h\}=\operatorname{span}\left\{\left(\begin{array}{llll}0 & 0 & 0 & 1\end{array}\right)^{T}\right\}$.

Suite au calcul des distributions :

$$
\begin{aligned}
& C_{*}^{P_{1}}=\operatorname{span}\left\{\left(\begin{array}{l}
1 \\
0 \\
0 \\
0
\end{array}\right),\left(\begin{array}{l}
0 \\
1 \\
0 \\
0
\end{array}\right),\left(\begin{array}{c}
0 \\
0 \\
x_{3}(t) \\
0
\end{array}\right)\right\} \quad C_{*}^{P_{2}}=\operatorname{span}\left\{\left(\begin{array}{l}
0 \\
1 \\
0 \\
0
\end{array}\right),\left(\begin{array}{c}
0 \\
0 \\
x_{3}(t) \\
0
\end{array}\right)\right\} \quad C_{*}^{P_{3}}=\operatorname{span}\left\{\left(\begin{array}{l}
0 \\
0 \\
0 \\
1
\end{array}\right),\left(\begin{array}{c}
0 \\
2 x_{3}(t) \\
0 \\
2
\end{array}\right),\left(\begin{array}{c}
0 \\
2 x_{3}(t) x_{4}(t) \\
1 \\
2 x_{4}(t)
\end{array}\right)\right\} \\
& S_{*}^{P_{1}}=\operatorname{span}\left\{\left(\begin{array}{l}
1 \\
0 \\
0 \\
0
\end{array}\right)\right\} \quad S_{*}^{P_{2}}=\operatorname{span}\left\{\left(\begin{array}{l}
0 \\
1 \\
0 \\
0
\end{array}\right)\right\} \quad S_{*}^{P_{3}}=\operatorname{span}\left\{\left(\begin{array}{l}
0 \\
0 \\
0 \\
1
\end{array}\right),\left(\begin{array}{c}
0 \\
2 x_{3}(t) \\
0 \\
2
\end{array}\right),\left(\begin{array}{c}
2 x_{3}(t) x_{4}(t) \\
1 \\
2 x_{4}(t)
\end{array}\right)\right\} \\
& \left(S_{*}^{P_{1}}\right)^{\perp}=\operatorname{span}\left\{\left(\begin{array}{l}
0 \\
1 \\
0 \\
0
\end{array}\right),\left(\begin{array}{l}
0 \\
0 \\
1 \\
0
\end{array}\right),\left(\begin{array}{l}
0 \\
0 \\
0 \\
1
\end{array}\right)\right\} \quad\left(S_{*}^{P_{2}}\right)^{\perp}=\operatorname{span}\left\{\left(\begin{array}{l}
1 \\
0 \\
0 \\
0
\end{array}\right),\left(\begin{array}{l}
0 \\
0 \\
1 \\
0
\end{array}\right),\left(\begin{array}{l}
0 \\
0 \\
0 \\
1
\end{array}\right)\right\} \quad\left(S_{*}^{P_{3}}\right)^{\perp}=\operatorname{span}\left\{\left(\begin{array}{l}
1 \\
0 \\
0 \\
0
\end{array}\right)\right\}
\end{aligned}
$$

l'application des méthodes développées dans le chapitre précédent montre que les défauts sont isolables et que la matrice $\mathcal{A}_{m}$ (définie par l'équation (2.78)) s'écrit :

$$
\mathcal{A}_{m}=\left(\begin{array}{lll}
1 & 0 & 0 \\
0 & 1 & 1 \\
0 & 0 & 1
\end{array}\right)
$$

La matrice est de rang plein, le théorème 2.12 est donc vérifié. Les suppositions de décomposition en sommes directes des distributions $S_{*}^{P_{j}}$ avec $j=\{1,2,3\}$ sont également satisfaites :

$$
\begin{aligned}
& S_{*}^{p_{1}}=\phi^{p_{1}}=\operatorname{span}\left\{\left(\begin{array}{llll}
1 & 0 & 0 & 0
\end{array}\right)^{T}\right\} \\
& S_{*}^{p_{2}}=\phi^{p_{2}, p_{3}}=\operatorname{span}\left\{\left(\begin{array}{llll}
0 & 1 & 0 & 0
\end{array}\right)^{T}\right\} \\
& S_{*}^{p_{3}}=\phi^{p_{3}} \oplus \phi^{p_{2}, p_{3}} \text { avec } \phi^{p_{3}}=\operatorname{span}\left\{\left(\begin{array}{llll}
0 & 0 & 1 & 0
\end{array}\right)^{T},\left(\begin{array}{llll}
0 & 0 & 0 & 1
\end{array}\right)^{T}\right\}
\end{aligned}
$$


nous définissons le difféomorphisme suivant :

$$
\tilde{x}(t)=\Phi(x(t)) \text { avec } \frac{\partial \Phi(x(t))}{\partial x(t)}=\left(\begin{array}{lll}
\phi^{p_{1}} & \phi^{p_{3}} & \phi^{p_{2}, p_{3}}
\end{array}\right)^{T}
$$

Dans cet exemple la distribution $\gamma$ n'est pas définie car l'ensemble des distributions $\phi^{p_{1}}, \phi^{p_{3}}$ et $\phi^{p_{2}, p_{3}}$ définissent l'espace d'état dans sa totalité.

Dans ces nouvelles coordonnées, le filtre s'écrit :

$$
\Sigma_{\widetilde{F N L}}:\left\{\begin{array}{l}
\dot{\tilde{z}}_{1}(t)=\tilde{z}_{1}(t)+u_{1}(t)+\Psi^{1}\left(\breve{z}_{*}^{1}(t), u(t), y_{x}(t)\right)-\Psi^{1}\left(\breve{z}_{*}^{1}(t), u(t), y_{z}(t)\right) \\
\dot{\tilde{z}}_{3}(t)=\left(\begin{array}{l}
\dot{\tilde{z}}_{31}(t) \\
\dot{\tilde{z}}_{32}(t)
\end{array}\right)=\left(\begin{array}{c}
\tilde{z}_{2,3}(t) \tilde{z}_{31}(t)+\tilde{z}_{32}(t) \\
\tilde{z}_{32}^{2}(t)+u_{3}(t)
\end{array}\right)+\Psi^{3}\left(\breve{z}_{*}^{3}(t), u(t), y_{x}(t)\right) \\
\left.\dot{\tilde{z}}_{2,3}(t)=\tilde{z}_{31}(t) \tilde{z}_{32}^{2}(t)+\tilde{z}_{*}^{3}(t), u(t), y_{z}(t)\right) \\
y_{z}(t)=\left[\tilde{z}_{1}^{2}(t) \tilde{z}_{2,3}(t) \tilde{z}_{31}(t) \tilde{z}_{31}(t)\right]^{T}
\end{array}\right.
$$

avec $\operatorname{dim}\left(\tilde{z}_{3}(t)\right)=\operatorname{dim}\left(\phi^{p_{3}}\right)=2$ et où les notations référencées lors de l'écriture du système (3.30) sont définies comme suit :

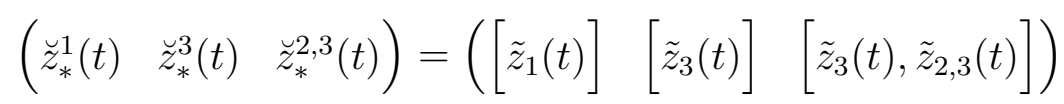

nous posons alors

$$
\breve{z}_{*}^{1}(t)=\tilde{z}_{1}(t) \quad ; \quad \breve{z}_{*}^{3}(t)=\left(\begin{array}{c}
\breve{z}_{* 1}^{3}(t) \\
\breve{z}_{* 2}^{3}(t)
\end{array}\right)=\left(\begin{array}{c}
\tilde{z}_{31}(t) \\
\tilde{z}_{32}(t)
\end{array}\right) \quad ; \quad \breve{z}_{*}^{2,3}(t)=\left(\begin{array}{c}
\breve{z}_{* 1}^{2,3}(t) \\
\breve{z}_{* 2}^{2,3}(t) \\
\breve{z}_{* 3}^{2,3}(t)
\end{array}\right)=\left(\begin{array}{c}
\tilde{z}_{31}(t) \\
\tilde{z}_{32}(t) \\
\tilde{z}_{2,3}(t)
\end{array}\right)
$$

et chacune des composantes de l'injection de sortie est définie afin de satisfaire aux caractéristiques d'invariance. Ainsi les égalités définies en (3.31) doivent être satisfaites :

$$
\left\{\begin{array}{l}
\breve{z}_{*}^{1}(t)+u_{1}(t)+\Psi^{1}\left(\breve{z}_{*}^{1}(t), u(t), y_{z}(t)\right)=\tilde{z}_{1}(t)+u_{1}(t) \\
\left(\begin{array}{c}
\breve{z}_{* 2}^{3}(t) \\
\left(\breve{z}_{* 2}^{3}\right)^{2}(t)+u_{3}(t)
\end{array}\right)+\Psi^{3}\left(\breve{z}_{*}^{3}(t), u(t), y_{z}(t)\right)=\left(\begin{array}{c}
\tilde{z}_{2,3}(t) \tilde{z}_{31}(t)+\tilde{z}_{32}(t) \\
\tilde{z}_{32}^{2}(t)+u_{3}(t)
\end{array}\right) \\
\breve{z}_{* 1}^{2,3}(t)\left(\breve{z}_{* 2}^{2,3}\right)^{2}(t)+\Psi^{2,3}\left(\breve{z}_{*}^{2,3}(t), u(t), y_{z}(t)\right)=\tilde{z}_{31}(t) \tilde{z}_{32}^{2}(t)+\tilde{z}_{1}(t) u_{2}(t)
\end{array}\right.
$$

nous en déduisons :

$$
\left\{\begin{array}{l}
\Psi^{1}\left(\breve{z}_{*}^{1}(t), u(t), y_{z}(t)\right)=0 \\
\Psi^{3}\left(\breve{z}_{*}^{3}(t), u(t), y_{z}(t)\right)=\left(\begin{array}{c}
y_{z 2}(t) \\
0
\end{array}\right) \\
\Psi^{2,3}\left(\breve{z}_{*}^{2,3}(t), u(t), y_{z}(t)\right)=\sqrt{y_{z 1}(t)} u_{2}(t)
\end{array}\right.
$$


et le filtre s'écrit comme suit dans le système de coordonnées d'origine :

$$
\Sigma_{F N L}:\left\{\begin{aligned}
\dot{z}_{1}(t) & =z_{1}(t)+u_{1}(t) \\
\dot{z}_{2}(t) & =z_{3}(t) z_{4}^{2}(t)+\sqrt{y_{x 1}(t)} u_{2}(t) \\
\dot{z}_{3}(t) & =y_{x 2}(t)+z_{4}(t) \\
\dot{z}_{4}(t) & =z_{4}^{2}(t)+u_{3}(t) \\
y_{z}(t) & =\left(z_{1}^{2}(t) z_{2}(t) z_{3}(t) z_{3}(t)\right)^{T}
\end{aligned}\right.
$$

Suite à ces différentes manipulations, nous assurons :

- l'insensibilité du sous-espace d'état associé à $z_{1}(t)$ à tout défaut autre que $w_{1}(t)$. $z_{1}(t)$ est en effet égal à $x_{1}(t) \forall w_{2}(t)$, et $w_{3}(t)$ si, et seulement si, $w_{1}(t)=0$.

- l'insensibilité du sous-espace d'état associé à $z_{2}(t)$ à tout défaut autre que $w_{2}(t)$ et $w_{3}(t)$. $z_{2}(t)$ est en effet égal à $x_{2}(t) \forall w_{1}(t)$ si, et seulement si, $w_{2}(t)=0$ et $w_{3}(t)=0$.

- l'insensibilité du sous-espace d'état associé à $\left(z_{3}(t) z_{4}(t)\right)$ à tout défaut autre que $w_{3}(t)$. $\left(z_{3}(t) z_{4}(t)\right)$ est en effet égal à $\left(x_{3}(t) x_{4}(t)\right) \forall w_{1}(t)$, et $w_{2}(t)$ si, et seulement si, $w_{3}(t)=0$. Une fois effectuée la synthèse du filtre, l'étape même de génération de résidus est nécessaire afin de finaliser l'étude.

Lors de l'analyse structurelle (chapitre 2) menant aux conditions d'isolation du vecteur défaut $w(t)$, la $y$-reconstructibilité a été considérée. Bien que le résidu $r_{j}(t)$ vérifie $\operatorname{span}\left\{d r_{j}^{T}\right\} \nsubseteq$ $\phi^{P_{j}}$, il est important de noter que la localisation est assurée par l'inclusion $\operatorname{span}\left\{d r_{j}^{T}\right\} \subseteq$ $\left(\phi^{P_{j}} \oplus \phi^{P_{j}, P_{i}} \oplus \cdots \oplus \phi^{\cdots, P_{j}, \cdots}\right)$. En effet, d'après la condition de rang énoncée au théorème 2.12 , il existe $q$ résidus permettant l'isolation des défauts avec la certitude que chacun d'entre eux est sensible à au moins un défaut différent du résidu précédent.

\subsubsection{Calcul de l'injection de sortie dans un cadre général}

Il semble particulièrement intéressant de développer le calcul ou le moyen de déterminer l'injection de sortie généralisée dans un cas multi-filtres. Ainsi dans cette section, la localisation des défauts est réalisée à l'aide d'un banc de $q$ filtres.

Cette présentation se justifie par l'intérêt qu'offre la synthèse de plusieurs filtres au problème du découplage total. En effet, comme nous l'avons déjà signalé au chapitre 2, un tel découplage est à proscrire lors de la recherche d'un seul filtre isolateur de défauts. En effet, dans ce cadre, il n'est pas avantageux de rendre insensible l'état estimé aux défauts. Ces derniers seraient ni détectables ni isolables. En revanche nous montrons que ce n'est pas le cas lors de la synthèse de plusieurs filtres.

L'énoncé du théorème 2.12 s'appuie sur l'étude du rang de la matrice $\mathcal{A}_{m}$ et énonce clairement les conditions d'isolation du vecteur défaut. L'analyse des inclusions des sous-espaces 
d'état représentés par les distributions $S_{m *}^{P_{j}}$ est à la base de notre réflexion.

Nous considérons le défaut $w_{j}(t)$ et nous cherchons à synthétiser le filtre dédié à son isolation. Cependant, nous précisons que l'étape de découplage total pour le calcul de $S_{m *}^{P_{j}}$ est inhibée, i.e. nous ne cherchons pas la nullité de $S_{m *}^{P_{j}}\left(\operatorname{span}\left\{P_{j}\right\} \subseteq S_{m *}^{P_{j}}\right)$. En revanche, plus $S_{m *}^{P_{k}}$ avec $k=\{1, \cdots, q\}$ et $k \neq j$ est "petit" (ou nul), moins l'inclusion de $\left(S_{m *}^{P_{k}}\right)^{\perp}$ dans $\left(S_{m *}^{P_{j}}\right)^{\perp}$ est probable. C'est la raison pour laquelle l'étape de découplage total n'est pas inhibée lors du calcul de $S_{m *}^{P_{k}}$. Le résidu résultant de cette étude doit être insensible au plus grand nombre de défauts tout en étant sensible au défaut $w_{j}(t)$.

Nous rappelons, ci-dessous, la forme de la matrice d'intersections (2.76) associée au seul défaut $w_{j}(t)$ :

$$
\mathcal{B}^{P_{j}}=\left(\begin{array}{c}
\left(\mathcal{B}^{P_{j}}\right)_{1} \\
\left(\mathcal{B}^{P_{j}}\right)_{2} \\
\vdots \\
\left(\mathcal{B}^{P_{j}}\right)_{\epsilon_{P_{j}}}
\end{array}\right) \in \mathbb{R}^{\epsilon_{P_{j}} \times q}
$$

où $\epsilon_{P_{j}}$ est le nombre de solutions du critère (2.75).

Bien que nous n'ayons pas trouvé de règles permettant de choisir une distribution parmi les $\epsilon_{P_{j}}$ distributions, certaines facilitent l'étude tant au niveau du découplage que lors de la génération du résidu.

Ainsi, nous notons $\Lambda^{P_{j}}$ la distribution choisie (arbitrairement ou non) et associée au défaut $w_{j}(t)$. Cette dernière vérifie une succession d'intersections :

$$
\Lambda^{P_{j}} \subseteq\left(S_{m *}^{P_{k 1}}\right)^{\perp} \cap \cdots \cap\left(S_{m *}^{P_{k 2}}\right)^{\perp} \cap\left(S_{m *}^{P_{k 3}}\right)^{\perp} \cap \cdots \cap\left(S_{m *}^{P_{k 4}}\right)^{\perp} \nsubseteq\left(S_{m *}^{P_{j}}\right)^{\perp}
$$

avec $k 1, \cdots, k 2, k 3, \cdots, k 4 \in\{1, \cdots, q\}$.

Dans le cadre de l'étude que nous menons et afin de préciser la démarche adoptée, nous distinguons les défauts faisant l'objet d'un découplage total des autres. C'est-à-dire que nous considérons les défauts $\left(\begin{array}{lll}w_{k 1}(t) & \cdots & w_{k 2}(t)\end{array}\right)$ non totalement découplables et les défauts $\left(w_{k 3}(t) \quad \cdots \quad w_{k 4}(t)\right)$ totalement découplables. Ainsi les sous-espaces d'état $S_{m *}^{P_{i}}$ pour $i=$ $\{k 3, \cdots, k 4\}$ sont réduits à l'ensemble nul.

En prenant l'exemple du découplage total du défaut $w_{k 3}(t)$, nous avons $S_{m *}^{P_{k 3}}=\{0\}$, ceci implique qu'aucune distribution $\phi^{\cdots,}, P_{k 3}, \cdots$ n'est définie ( $c f$. paragraphe 3.2.1.2).

De même que dans les paragraphes précédents, nous supposons :

$$
S_{m *}^{P_{j}}=\phi^{P_{j}} \oplus \phi^{P_{j}, P_{i}} \oplus \cdots \oplus \phi^{\cdots, P_{j}, \cdots}
$$

où $\{j\},\{i, j\}$ et $\{\cdots, j, \cdots\}$ sont des sous-ensembles de $\{k 1, \cdots, k 2\}$.

Lors de la synthèse du filtre dédié à l'isolation du défaut $w_{j}(t)$, seules les distributions $S_{m *}^{P_{k 1}}$, $\cdots, S_{m *}^{P_{k 2}}$ doivent satisfaire l'hypothèse de décomposition en somme directe. En ce sens, il peut être avantageux de synthétiser un banc de filtres. Rappelons que dans les deux sections précédentes, toutes les distributions $\left(S_{*}^{P_{j}}, j=\{1, \cdots, q\}\right)$ doivent satisfaire cette hypothèse. 
Suite à ces définitions, nous considérons le changement de coordonnées suivant nécessaire à la détermination de l'injection de sortie :

$$
\frac{\partial \tilde{x}(t)}{\partial x(t)}=\frac{\partial \Phi(x(t))}{\partial x(t)}=\left(\begin{array}{llllllll}
\phi^{P_{k 5}} & \cdots & \phi^{P_{k 6}} & \phi^{P_{k 7}, P_{k 8}} & \cdots & \phi^{P_{k 9}, \cdots, P_{k 10}} & \Lambda^{P_{j}} & \gamma
\end{array}\right)^{T}
$$

où $\phi^{\bullet}$ est le résultat de la décomposition des distributions $S_{m *}^{P_{k 1}}, \cdots, S_{m *}^{P_{k 2}}$ donc $k 5, \cdots, k 10 \in\{k 1, \cdots, k 2\}$. $\gamma$ est choisi de manière à assurer l'intégrabilité de $\Phi(x(t))$ et est orthogonale à toute autre distribution définissant le difféomorphisme.

Il faut remarquer que $\Lambda^{P_{j}}$, d'après la contrainte (3.46), est nécessairement orthogonale au plus petit sous-espace d'état sensible aux défauts $\left(\begin{array}{lllllll}w_{k 1}(t) & \cdots & w_{k 2}(t) & w_{k 3}(t) & \cdots & w_{k 4}(t)\end{array}\right)$, soit $\Lambda^{P_{j}} \subseteq\left(\phi^{\bullet}\right)^{\perp}$. Remarquons que ce difféomorphisme ne joue aucun rôle dans l'expression de l'injection de sortie dédiée au découplage total des défauts $w_{i}(t), i=\{k 3, \cdots, k 4\}$. En effet, cette expression est implicite suite à l'estimation des défauts $w_{i}(t)$.

La distinction de traitement réalisée entre les indices $\{k 1, \cdots, k 2\}$ (découplage classique) et $\{k 3, \cdots, k 4\}$ (découplage total) se prolonge donc dans la formulation de l'injection de sortie. En effet,

- lors d'un découplage total, l'injection de sortie est de la forme suivante :

$$
\Psi\left(\bullet_{x}(t), \bullet_{z}(t), \bullet_{u}(t)\right)=\Psi_{d t}\left(\Delta_{x}\right)
$$

avec

$$
\Delta_{x}=\left[Y_{x}(t), u(t), \cdots, u^{(\max -1)}(t), \xi_{1}\left(Y_{x}(t), Y_{x 1}(t)\right), \cdots, \xi_{l}\left(Y_{x}(t), Y_{x l}(t)\right), \tilde{z}_{N 2}(t)\right]
$$

- et lors d'un découplage classique :

$$
\Psi\left(\bullet_{x}(t), \bullet_{z}(t), \bullet_{u}(t)\right)=\Psi_{d c}\left(\Delta_{x}\right)-\Psi_{d c}\left(\Delta_{z}\right)
$$

avec $\Delta_{x}$ défini comme précédemment et

$$
\begin{gathered}
\Delta_{z}=\left[Y_{z}(t), u(t), \cdots, u^{(\max -1)}(t), \xi_{1}\left(Y_{z}(t), Y_{z 1}(t)\right), \cdots, \xi_{l}\left(Y_{z}(t), Y_{z l}(t)\right), \tilde{z}_{N 2}(t)\right] \\
Y_{\bullet}(t)=\left[y_{\bullet 1}^{(0)}(t), \cdots, y_{\bullet 1}^{\left(\min _{j}\left(\rho_{m 1}^{w_{j}}\right)-1\right)}(t), \cdots, y_{\bullet p}^{(0)}(t), \cdots, y_{\bullet p}^{\left(\min _{j}\left(\rho_{m p}^{w_{j}}\right)-1\right)}(t)\right] \text { avec } \bullet=x(t) \text { ou } y(t), \\
Y_{\bullet}(t), \cdots, Y_{\bullet}(t) \text { des signaux constitués de dérivations des sorties d'indices supérieurs aux } \\
\left(\min _{j}\left(\rho_{m i}^{w_{j}}\right)-1\right) \text {. Les fonctions } \xi_{1}(\bullet), \cdots, \xi_{l}(\bullet) \text { ne font pas intervenir dans leurs écritures } \\
\text { les défauts ou leurs dérivations et max rappelle l'indice de dérivation maximal des sorties. }
\end{gathered}
$$

Ainsi, dans cette section, nous cherchons l'expression des injections de sortie en fonction des types de découplage effectués (classique ou total). Dans le nouveau système de coordonnées 
défini par le difféomorphisme (3.48), nous écrivons les filtres suivants $(j=\{1, \cdots, q\})$ :

$\Sigma_{\widetilde{F N L}}:\left\{\begin{array}{l}\dot{\tilde{z}}_{k 5}(t)=\tilde{f}_{0}^{k 5}\left(\tilde{z}_{*}^{k 5}(t)\right)+\sum_{i=0}^{m} \tilde{f}_{i}^{k 5}\left(\tilde{z}_{*}^{k 5}(t)\right) u_{i}(t)+\tilde{\Psi}^{k 5}\left(\bullet_{x}(t), \bullet_{z}(t), \bullet_{u}(t)\right) \\ \vdots \\ \dot{\tilde{z}}_{k 6}(t)=\tilde{f}_{0}^{k 6}\left(\tilde{z}_{*}^{k 6}(t)\right)+\sum_{i=0}^{m} \tilde{f}_{i}^{k 6}\left(\tilde{z}_{*}^{k 6}(t)\right) u_{i}(t)+\tilde{\Psi}^{k 6}\left(\bullet_{x}(t), \bullet_{z}(t), \bullet_{u}(t)\right) \\ \dot{\tilde{z}}_{k 7, k 8}(t)=\tilde{f}_{0}^{k 7, k 8}\left(\tilde{z}_{*}^{k 7, k 8}(t)\right)+\sum_{i=0}^{m} \tilde{f}_{i}^{k 7, k 8}\left(\tilde{z}_{*}^{k 7, k 8}(t)\right) u_{i}(t)+\tilde{\Psi}^{k 7, k 8}\left(\bullet_{x}(t), \bullet_{z}(t), \bullet_{u}(t)\right) \\ \quad+\tilde{\tilde{z}}_{k 9, \cdots, k 10}(t)=\breve{f}_{0}^{k 9, \cdots, k 10}\left(\breve{z}_{*}^{k 9, \cdots, k 10}(t)\right)+\sum_{i=0}^{m} \breve{f}_{i}^{k 9, \cdots, k 10}\left(\tilde{z}_{*}^{k 9, \cdots, k 10}(t)\right) u_{i}(t) \\ \dot{\tilde{z}}_{\Lambda^{P j}}(t)=\tilde{f}_{0}^{\Lambda^{P_{j}}}\left(\breve{z}_{*}^{\Lambda^{P_{j}}}(t)\right)+\sum_{i=0}^{m} \tilde{f}_{i}^{\Lambda^{P_{j}}}\left(\breve{z}_{*}^{\Lambda^{P_{j}}}(t)\right) u_{i}(t)+\tilde{\Psi}^{\Lambda^{P_{j}}}\left(\bullet_{x}(t), \bullet_{z}(t), \bullet_{u}(t)\right) \\ \dot{\tilde{z}}_{\gamma}(t)=\tilde{f}_{0}^{\gamma}\left(\breve{z}_{*}^{\gamma}(t)\right)+\sum_{i=0}^{m} \tilde{f}_{i}^{\gamma}\left(\tilde{z}_{*}^{\gamma}(t)\right) u_{i}(t)+\tilde{\Psi}^{\gamma}\left(\bullet_{x}(t), \bullet_{z}(t), \bullet_{u}(t)\right) \\ \left.y_{z}(t)=\tilde{h}_{(\tilde{z}}(t)\right)\end{array}\right.$

avec les notations :

$$
\begin{aligned}
& \left(\begin{array}{lllllll}
\breve{z}_{*}^{k 5}(t) & \cdots & \breve{z}_{*}^{k 6}(t) & \breve{z}_{*}^{k 7, k 8}(t) & \cdots & \breve{z}^{k 9, \cdots, k 10}(t) & \breve{z}_{*}^{\Lambda^{P_{j}}}(t) \quad \breve{z}_{*}^{\gamma}(t)
\end{array}\right)= \\
& \left(\left[\tilde{z}_{k 5}(t), \tilde{z}_{\Lambda^{P_{j}}}(t), \tilde{z}_{\gamma}(t)\right] \cdots \quad\left[\tilde{z}_{k 6}(t), \tilde{z}_{\Lambda^{P_{j}}}(t), \tilde{z}_{\gamma}(t)\right]\right. \\
& {\left[\tilde{z}_{k 7}(t), \tilde{z}_{k 8}(t), \tilde{z}_{k 7, k 8}(t), \tilde{z}_{\Lambda^{P_{j}}}(t), \tilde{z}_{\gamma}(t)\right] \quad \cdots \quad\left[\tilde{z}_{k 9}(t), \cdots, \quad \tilde{z}_{k 10}(t), \tilde{z}_{k 9, \cdots, k 10}(t), \tilde{z}_{\Lambda^{P_{j}}}(t), \tilde{z}_{\gamma}(t)\right]} \\
& \left.\left[\tilde{z}_{\Lambda^{P_{j}}}(t), \tilde{z}_{\gamma}(t)\right] \quad\left[\tilde{z}_{\Lambda^{P_{j}}}(t), \tilde{z}_{\gamma}(t)\right]\right)
\end{aligned}
$$

Evidemment chaque formulation de l'injection de sortie doit comporter une partie liée au découplage total et une partie liée au découplage classique :

$$
\begin{aligned}
\tilde{\Psi}^{\bullet}\left(\bullet_{x}(t), \bullet_{z}(t), \bullet_{u}(t)\right) & =\tilde{\Psi}_{d t}^{\bullet}\left(\bullet_{x}(t), \bullet_{z}(t), \bullet_{u}(t)\right)+\tilde{\Psi}_{d c}^{\bullet}\left(\bullet_{x}(t), \bullet_{z}(t), \bullet_{u}(t)\right) \\
& =\tilde{\Psi}_{d t}^{\bullet}\left(\Delta_{x}\right)+\tilde{\Psi}_{d c}^{\bullet}\left(\Delta_{x}\right)-\tilde{\Psi}_{d c}^{\bullet}\left(\Delta_{z}\right)
\end{aligned}
$$

Les conditions d'invariance ne sont vérifiées que si l'injection de sortie liée au découplage classique satisfait l'égalité suivante :

$$
\begin{gathered}
\breve{f}_{0}^{\bullet}\left(\breve{z}_{*}^{\bullet}(t)\right)+\sum_{i=0}^{m} \breve{f}_{i}^{\bullet}\left(\breve{z}_{*}^{\bullet}(t)\right) u_{i}(t)+\tilde{\Psi}_{d c}^{\bullet}\left(\Delta_{z}\right)=\tilde{f}_{0}^{\bullet}(\tilde{z}(t))+\sum_{i=0}^{m} \tilde{f}_{i}^{\bullet}(\tilde{z}(t)) u_{i}(t) \\
\Downarrow \\
\tilde{\Psi}_{d c}^{\bullet}\left(\Delta_{z}\right)=\tilde{f}_{0}^{\bullet}(\tilde{z}(t))+\sum_{i=0}^{m} \tilde{f}_{i}^{\bullet}(\tilde{z}(t)) u_{i}(t)-\left(\breve{f}_{0}^{\bullet}\left(\breve{z}_{*}^{\bullet}(t)\right)+\sum_{i=0}^{m} \breve{f}_{i}^{\bullet}\left(\breve{z}_{*}^{\bullet}(t)\right) u_{i}(t)\right)
\end{gathered}
$$


avec $\bullet=\left\{[k 5], \cdots,[k 6],[k 7, k 8], \cdots,[k 9, \cdots, k 10],\left[\Lambda^{P_{j}}\right],[\gamma]\right\}$.

De cette écriture (3.56), $\tilde{\Psi}_{d c}^{\bullet}\left(\Delta_{x}\right)$ est déduite.

Concernant l'injection de sortie liée au découplage total, puisque son existence implique $\Psi_{d t}^{\bullet}\left(\Delta_{x}\right)=\sum_{i=k 3, \cdots, k 4} P_{i} w_{i}(t)$, son expression est implicite à partir de l'effet des défauts $w_{i}(t)$.

\section{Exemples académiques}

Nous proposons la synthèse du filtre associé à l'étude réalisée dans le paragraphe 2.3.5.1. Dans cette section, nous avions proposé le découplage d'un défaut de dimension deux à l'aide, d'une injection de sortie généralisée (utilisant les dérivées des sorties). Nous considérons dans cet exemple la synthèse d'un filtre assurant l'isolation d'un hypothétique défaut $w_{j}(t)$. Nous rappelons l'écriture du système défini en (2.66) :

$$
\Sigma_{N L}\left\{\begin{array}{l}
\dot{x}_{1}(t)=-x_{1}^{2}(t)+u_{1}(t)+w_{1}(t) \\
\dot{x}_{2}(t)=-x_{2}(t)+x_{3}(t) u_{2}(t) \\
\dot{x}_{3}(t)=x_{2}(t)-x_{3}(t) x_{2}(t)^{2} w_{2}(t)+u_{3}(t) \\
\dot{x}_{4}(t)=-x_{3}(t) x_{4}(t) \\
y_{x 1}(t)=x_{1}(t) \\
y_{x 2}(t)=x_{4}(t)
\end{array}\right.
$$

Nous avons montré que $S_{m *}^{P_{1}}=\{0\}$ et $S_{m *}^{P_{2}}=\left\{\left(\begin{array}{llll}0 & 0 & 1 & 0\end{array}\right)^{T}\right\}$. Ainsi en appliquant la procédure développée dans le paragraphe précédent :

- l'ensemble des indices $\{k 1, \cdots, k 2\}$ est réduit à $\{2\}$ et représente l'indice du défaut non estimable

- l'ensemble des indices $\{k 3, \cdots, k 4\}$ est réduit à $\{1\}$ et représente l'indice du défaut estimable et donc totalement découplable.

Seul le défaut $w_{1}(t)$ est estimable donc seule la distribution $S_{m *}^{P_{2}}$ doit être décomposable en somme directe. Cette propriété est nécessairement satisfaite puisque dans cet exemple :

$$
S_{m *}^{P_{2}}=\phi^{P_{2}}
$$

Ainsi toute distribution de $\left(S_{m *}^{P_{2}}\right)^{\perp}$ est insensible aux défauts $w_{1}(t)$ et $w_{2}(t)$. Arbitrairement nous choisissons :

$$
\Lambda^{P_{j}}=\operatorname{span}\left\{\left(\begin{array}{llll}
1 & 0 & 0 & 0
\end{array}\right)^{T}\right\}
$$

Le difféomorphisme qui définit le changement de coordonnées s'écrit comme suit :

$$
\tilde{x}(t)=\Phi(x(t))=\left(\begin{array}{c}
\left(\phi^{P_{2}}\right)^{T} \\
\left(\Lambda^{P_{j}}\right)^{T} \\
(\gamma)^{T}
\end{array}\right)=\left(\begin{array}{llll}
0 & 0 & 1 & 0 \\
1 & 0 & 0 & 0 \\
0 & 1 & 0 & 0 \\
0 & 0 & 0 & 1
\end{array}\right)
$$


Dans ce système de coordonnées, nous définissons le filtre suivant :

$$
\Sigma_{\widetilde{F N L}}\left\{\begin{array}{l}
\dot{\tilde{z}}_{1}(t)=\tilde{z}_{\gamma 1}(t)+u_{3}(t)+\tilde{\Psi}^{1}\left(\bullet_{x}(t), \bullet_{z}(t), \bullet_{u}(t)\right) \\
\dot{\tilde{z}}_{\Lambda^{P_{j}}}(t)=-\tilde{z}_{\Lambda^{P_{j}}}(t)+u_{1}(t)+\tilde{\Psi}^{2}\left(\bullet_{x}(t), \bullet_{z}(t), \bullet_{u}(t)\right) \\
\dot{\tilde{z}}_{\gamma}(t)=\left(\begin{array}{l}
\dot{\tilde{z}}_{\gamma 1}(t) \\
\dot{\tilde{z}}_{\gamma 2}(t)
\end{array}\right)=\left(\begin{array}{c}
-\tilde{z}_{\Lambda^{P_{j}}}(t)+\tilde{z}_{1}(t) u_{2}(t) \\
-\tilde{z}_{1}(t) \tilde{z}_{\gamma 2}(t)
\end{array}\right)+\tilde{\Psi}^{3}\left(\bullet_{x}(t), \bullet_{z}(t), \bullet_{u}(t)\right) \\
y_{z 1}(t)=\tilde{z}_{\Lambda^{P_{j}}}(t) \\
y_{z 2}(t)=\tilde{z}_{\gamma 2}(t)
\end{array}\right.
$$

Rappelons que chaque injection de sortie est la concaténation d'une injection visant à l'estimation d'une partie du défaut $\Psi_{d t}\left(\Delta_{x}\right)$ et d'une injection ayant pour objectif le découplage de l'autre partie du défaut $\Psi_{d c}\left(\Delta_{x}\right)$.

En précisant que le défaut $w_{1}(t)$ est $\Delta_{x}$-reconstructible $\left(w_{1}(t)=y_{x 1}^{(1)}(t)+y_{x 1}^{2}(t)-u_{1}(t)\right)$ et suite aux notations définies lors de l'étude théorique, nous précisons les différentes composantes de l'injection de sortie :

$$
\begin{aligned}
\tilde{\Psi}^{1}\left(\bullet_{x}(t), \bullet_{z}(t), \bullet_{u}(t)\right) & =\tilde{\Psi}_{d c}^{1}\left(\Delta_{x}\right)-\tilde{\Psi}_{d c}^{1}\left(\Delta_{z}\right) \\
\tilde{\Psi}^{2}\left(\bullet_{x}(t), \bullet_{z}(t), \bullet_{u}(t)\right) & =\tilde{\Psi}_{d t}^{2}\left(\Delta_{x}\right)+\tilde{\Psi}_{d c}^{2}\left(\Delta_{x}\right)-\tilde{\Psi}_{d c}^{2}\left(\Delta_{z}\right) \\
& =y_{x 1}^{(1)}(t)+y_{x 1}^{2}(t)-u_{1}(t)+\tilde{\Psi}_{d c}^{2}\left(\Delta_{x}\right)-\tilde{\Psi}_{d c}^{2}\left(\Delta_{z}\right) \\
\tilde{\Psi}^{3}\left(\bullet_{x}(t), \bullet_{z}(t), \bullet_{u}(t)\right) & =\tilde{\Psi}_{d c}^{3}\left(\Delta_{x}\right)-\tilde{\Psi}_{d c}^{3}\left(\Delta_{z}\right)
\end{aligned}
$$

La transformation choisie implique les notations suivantes :

$$
\left(\breve{z}_{*}^{1}(t) \quad \breve{z}_{*}^{\Lambda^{P_{j}}}(t) \quad \breve{z}_{*}^{\gamma}(t)\right)=\left(\left[\tilde{z}_{1}(t), \tilde{z}_{\Lambda^{P_{j}}}(t), \tilde{z}_{\gamma}(t)\right] \quad\left[\tilde{z}_{\Lambda^{P_{j}}}(t), \tilde{z}_{\gamma}(t)\right] \quad\left[\tilde{z}_{\Lambda^{P_{j}}}(t), \tilde{z}_{\gamma}(t)\right]\right)
$$

En appliquant la méthode de détermination explicite des injections de sorties, nous obtenons :

$$
\left\{\begin{array}{l}
\tilde{z}_{\gamma 1}(t)+u_{3}(t)+\tilde{\Psi}_{d c}^{1}\left(\Delta_{z}\right)=\tilde{z}_{\gamma 1}(t)+u_{3}(t) \\
-\tilde{z}_{\Lambda^{P_{j}}}^{2}(t)+u_{1}(t)+\tilde{\Psi}_{d c}^{2}\left(\Delta_{z}\right)=-\tilde{z}_{\Lambda^{P_{j}}}^{2}(t)+u_{1}(t) \\
\left(\begin{array}{c}
-\tilde{z}_{\Lambda^{P_{j}}}(t) \\
0
\end{array}\right)+\tilde{\Psi}_{d c}^{3}\left(\Delta_{z}\right)=\left(\begin{array}{c}
-\tilde{z}_{\Lambda^{P_{j}}}(t)+\tilde{z}_{1}(t) u_{2}(t) \\
-\tilde{z}_{1}(t) \tilde{z}_{\gamma 2}(t)
\end{array}\right)
\end{array}\right.
$$

et nous en déduisons :

$$
\left\{\begin{array}{l}
\tilde{\Psi}_{d c}^{1}\left(\Delta_{z}\right)=0 \\
\tilde{\Psi}_{d c}^{2}\left(\Delta_{z}\right)=0 \\
\tilde{\Psi}_{d c}^{3}\left(\Delta_{z}\right)=\left(\begin{array}{c}
\frac{-y_{z 2}^{(1)}(t)}{y_{z 2}(t)} u_{2}(t) \\
\frac{y_{z 2}^{(1)}(t)}{y_{z 2}(t)} \tilde{z}_{\gamma 2}(t)
\end{array}\right)
\end{array}\right.
$$


Dans la base d'origine, le filtre s'écrit comme suit :

$$
\Sigma_{N L}\left\{\begin{array}{l}
\dot{z}_{1}(t)=-z_{1}^{2}(t)+u_{1}(t)+y_{x 1}^{(1)}(t)+y_{x 1}^{2}(t)-u_{1}(t) \\
\dot{z}_{2}(t)=-z_{2}(t)+\left(\frac{-y_{x 2}^{(1)}(t)}{y_{x 2}(t)}\right) u_{2}(t) \\
\dot{z}_{3}(t)=z_{2}(t)+u_{3}(t) \\
\dot{z}_{4}(t)=\left(\frac{y_{x 2}^{(1)}(t)}{y_{x 2}(t)}\right) z_{4}(t) \\
y_{z 1}(t)=z_{1}(t) \\
y_{z 2}(t)=z_{4}(t)
\end{array}\right.
$$

Suite à ces différentes manipulations, nous assurons :

- l'insensibilité du sous-espace d'état tout entier à tout défaut autre que $w_{2}(t) . z(t)$ est en effet égal à $x(t) \forall w_{1}(t)$ si, et seulement si, $w_{2}(t)=0$.

- l'insensibilité du sous-espace d'état associé à $\left(z_{1}(t) z_{2}(t) z_{4}(t)\right)$ à tout défaut. $\left(z_{1}(t) z_{2}(t) z_{4}(t)\right)$ est égal à $\left(x_{1}(t) \quad x_{2}(t) \quad x_{4}(t)\right), \forall w_{1}(t)$ et $w_{2}(t)$.

Une fois la synthèse du filtre effectuée, l'étape même de génération de résidus est nécessaire afin de finaliser l'étude.

En supposant que le théorème 2.12 est vérifié, il existe toujours une distribution $d r_{j}(\forall j=$ $1, \cdots, q)$ telle que :

- les conditions de sensibilité sont satisfaites $\operatorname{span}\left\{d r_{j}^{T}\right\} \subseteq \Lambda^{P_{j}}=\left(S_{m *}^{P_{k 1}}\right)^{\perp} \cap \cdots \cap\left(S_{m *}^{P_{k 2}}\right)^{\perp} \cap$ $\left(S_{m *}^{P_{k 3}}\right)^{\perp} \cap \cdots \cap\left(S_{m *}^{P_{k 4}}\right)^{\perp} \nsubseteq\left(S_{m *}^{P_{j}}\right)^{\perp}$

- la distribution $d r_{j}$ est $y$-reconstructible et permet la synthèse du signal $r_{j}(t)$.

Ces différentes propositions déterminent précisément la forme du filtre assurant l'isolation du défaut $w_{j}(t)$. L'itération de ces étapes $(j=\{1, \cdots, q\})$ mène à la synthèse de $q$ filtres. Le résidu $r_{j}(t)$ provenant du $j^{\mathrm{e}}$ filtre permet d'isoler le défaut $w_{j}(t)$.

Dans ce paragraphe, nous avons déterminé une injection de sortie assurant l'invariance des sous-espaces d'état sensibles aux défauts.

Dans les chapitres précédents, nous avons supposé connaître précisément l'état initial du système et ainsi posé $x(t=0)=z(t=0)$. Sous cette hypothèse, nous assurons en l'absence de défauts l'égalité $x(t)=z(t), \forall t$ (rappelons que le modèle du système est parfait). En revanche, en nous affranchissant de cette hypothèse $(x(t=0) \neq z(t=0))$, il est particulièrement important, et ceci d'autant plus pour le diagnostic de système, de garantir la convergence $x(t) \rightarrow z(t)$ lorsque $t \rightarrow+\infty$. Cette discussion fait l'objet du paragraphe suivant. 


\subsection{Calcul de l'injection de sortie pour la convergence}

Lors de l'étude des systèmes linéaires, prouver la convergence des estimations joue un rôle d'importance lors de la synthèse d'observateurs ou de filtres. Cette étude assure la convergence vers zéro des résidus $r(t)=y_{x}(t)-y_{z}(t)$ en l'absence de défauts malgré une mauvaise initialisation de l'observateur ou du filtre : $\forall \varepsilon>0, \exists t$ tel que $\|r(t)\|<\varepsilon$.

Bien que l'intérêt de cette caractéristique ne soit plus à prouver, il reste difficile, dans le domaine des systèmes non linéaires de garantir la convergence. Par conséquent, nous nous proposons d'appliquer au domaine du diagnostic l'approche intuitive connue sous le nom d'analyse de contraction ou théorie de la contraction.

\subsubsection{Introduction à la théorie de la contraction}

L'étude de la convergence est souvent envisagée au travers de l'utilisation de fonctions de Lyapounov qui, bien que largement utilisées dans la littérature, présentent l'inconvénient de requérir la description explicite d'un mouvement nominal à partir duquel les propriétés de convergence sont étudiées. En outre, ceci conduit souvent à des expressions de l'erreur d'estimation parfois difficiles à exprimer de manière simple. Dans la théorie de la contraction ([49]), l'analyse de convergence des différentes trajectoires s'effectue de manière différentielle et sans nécessiter la connaissance du mouvement nominal. Ce type d'approche se révèle relativement simple pour analyser des techniques de commande et d'applications $([49,44])$ faisant intervenir le concept de relation entre trajectoires (tracking, observateurs, filtres).

Dans cette section, nous décrivons les fondements de la théorie de la contraction. Dans l'article original de W. Lohmiller et J-J. Slotine ([49]), le système non linéaire considéré s'écrit comme suit :

$$
\dot{x}(t)=f(x(t), t)
$$

En supposant $f(x(t), t)$ continûment différentiable, l'équation (3.67) vérifie :

$$
\delta \dot{x}=\frac{\partial f(x(t), t)}{\partial x(t)} \delta x
$$

avec $\delta x$ un déplacement virtuel infinitésimal défini à un instant donné.

En considérant deux trajectoires voisines vérifiant l'équation différentielle $\dot{x}(t)=f(x(t), t)$, nous représentons à la figure 3.3 le déplacement virtuel.

Le terme quadratique $\delta x^{T} \delta x$ peut être défini par :

$$
\frac{d}{d t}\left(\delta x^{T} \delta x\right)=2 \delta x^{T} \delta \dot{x}=2 \delta x^{T} \frac{\partial f(x(t), t)}{\partial x(t)} \delta x
$$




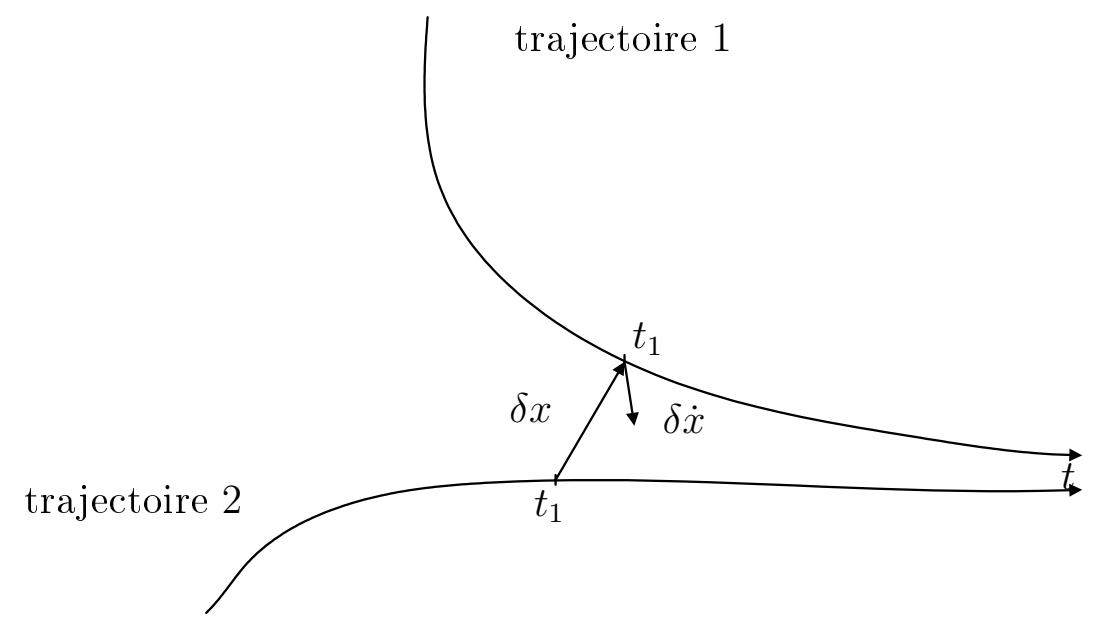

FIG. 3.3: Trajectoires et déplacements virtuels

pour lequel nous remarquons que :

$$
\frac{d}{d t}\left(\delta x^{T} \delta x\right)=\frac{d}{d t}\left(\delta x^{T} \delta x\right)^{T}=2 \delta x^{T}\left(\frac{\partial f(x(t), t)}{\partial x(t)}\right)^{T} \delta x
$$

Ainsi si $\lambda_{\max }(x, t)$ est la plus grande valeur propre de la partie symétrique de la matrice jacobienne $\partial f(x(t), t) / \partial x(t)\left(0.5\left((\partial f(x(t), t) / \partial x(t))+(\partial f(x(t), t) / \partial x(t))^{T}\right)\right)$ et en supposant que $\lambda_{\max }(x(t), t)$ est uniformément négative alors toute longueur infinitésimale $\|\delta x\|$ converge exponentiellement vers zéro. Cependant nous précisons que le taux de décroissance est majoré sans être maîtrisé. En effet, ce dernier varie le long de la trajectoire suivie par le système. Ainsi, nous rappelons la définition d'une région de contraction ([49]) :

Définition 3.1 Etant donné un système d'équations $\dot{x}(t)=f(x(t), t)$, une région de l'espace d'état est appelée région de contraction si la matrice jacobienne $\partial f(x(t), t) / \partial x(t)$ est définie uniformément négative dans cette région.

Par $\partial f(x(t), t) / \partial x(t)$ défini uniformément négatif, nous entendons :

$$
\exists \beta>0, \forall x, \forall t \geq 0, \frac{1}{2}\left(\frac{\partial f(x(t), t)}{\partial x(t)}+\frac{\partial f^{T}(x(t), t)}{\partial x(t)}\right) \leq-\beta I<0 .
$$

où $I$ est une matrice identité de dimension appropriée. Cependant pour des raisons calculatoires, il peut s'avérer judicieux de réaliser un changement de coordonnées :

$$
\delta z=\Theta(x(t), t) \delta x
$$

où $\Theta(x(t), t)$ est une matrice carrée.

L'évolution temporelle de $\delta z$ peut être déduite :

$$
\begin{aligned}
\frac{d}{d t} \delta z & =\dot{\Theta} \delta x+\Theta \dot{\delta} x \\
& =\left(\dot{\Theta}+\Theta \frac{\partial f(x(t), t)}{\partial x(t)}\right) \Theta^{-1} \delta z=F \delta z
\end{aligned}
$$


où la matrice jacobienne généralisée $F$ :

$$
F=\left(\dot{\Theta}+\Theta \frac{\partial f(x(t), t)}{\partial x(t)}\right) \Theta^{-1}
$$

s'associe à l'écriture suivante :

$$
\frac{d}{d t}\left(\delta z^{T} \delta z\right)=2 \delta z^{T} F \delta z
$$

D'une manière similaire à ce que nous avons décrit précédemment, la convergence exponentielle vers zéro de $\delta z$ (et donc de $\delta x$ ) est assurée dans la région où $F$ est définie uniformément négative. En rappelant la notation :

$$
\delta z^{T} \delta z=\delta x^{T} M \delta x
$$

où $M(x(t), t)=\Theta^{T} \Theta$ représente une métrique symétrique et continûment différentiable.

L'équation (3.73) peut alors se récrire dans les coordonnées $\delta x$ :

$$
\frac{d}{d t}\left(\delta x^{T} M \delta x\right)=\delta x^{T}\left(\frac{\partial f^{T}(x(t), t)}{\partial x(t)} M+\dot{M}+M \frac{\partial f(x(t), t)}{\partial x(t)}\right) \delta x
$$

Une autre définition de la contraction peut également être donnée (cf. [49]) :

Définition 3.2 Soit l'équation du système $\dot{x}(t)=f(x(t), t)$, une région de l'espace d'état est appelée région de contraction avec une métrique $M(x, t)$ définie uniformément positive si $F$ (équation (3.74)) ou $\left(\frac{\partial f^{T}(x(t), t)}{\partial x(t)} M+\dot{M}+M \frac{\partial f(x(t), t)}{\partial x(t)}\right)$ est définie uniformément négative dans cette région.

Bien que la définition d'une métrique permette de nombreuses possibilités, pour des raisons de commodité de calcul, il peut s'avérer plus avantageux d'étudier les connections (ou combinaisons) entre des sous-systèmes contractants. Nous rappelons à présent les termes originaux utilisés par W. Lohmiller et J.-J. E. Slotine [49].

\subsubsection{1 "Parallel combination"}

Nous considérons deux systèmes de mêmes dimensions connectés en parallèle :

$$
\begin{aligned}
& \dot{x}_{1}(t)=f_{1}\left(x_{1}(t), t\right) \\
& \dot{x}_{2}(t)=f_{2}\left(x_{2}(t), t\right)
\end{aligned}
$$

avec des dynamiques virtuelles définies comme suit :

$$
\begin{aligned}
& \delta \dot{z}_{1}=F_{1} \delta z \\
& \delta \dot{z}_{2}=F_{2} \delta z
\end{aligned}
$$

Si les deux systèmes sont contractants dans la même métrique alors n’importe quelle superposition est définie uniformément négative :

$$
\alpha_{1}(t) \delta \dot{z}_{1}+\alpha_{2}(t) \delta \dot{z}_{2}
$$

où $\exists \alpha>0, \forall t \geq 0, \alpha_{i}(t) \geq \alpha$. 


\subsubsection{2 "Feedback combination"}

D'une manière similaire, nous définissons la connection entre deux systèmes de dimension non nécessairement identiques :

$$
\begin{aligned}
& \dot{x}_{1}(t)=f_{1}\left(x_{1}(t), x_{2}(t), t\right) \\
& \dot{x}_{2}(t)=f_{2}\left(x_{1}(t), x_{2}(t), t\right)
\end{aligned}
$$

avec une combinaison dite en "feedback" :

$$
\frac{d}{d t}\left(\begin{array}{l}
\delta z_{1} \\
\delta z_{2}
\end{array}\right)=\left(\begin{array}{cc}
F_{1} & G \\
-G^{T} & F_{2}
\end{array}\right)\left(\begin{array}{l}
\delta z_{1} \\
\delta z_{2}
\end{array}\right)
$$

Le système augmenté est contractant si, et seulement si, les sous-systèmes séparés sont contractants. Ce qui implique que les matrices $F_{1}$ et $F_{2}$ soient définies uniformément négatives.

\subsubsection{3 "Hierarchical combination"}

Considérons la dynamique des déplacements virtuels définie comme suit :

$$
\frac{d}{d t}\left(\begin{array}{l}
\delta z_{1} \\
\delta z_{2}
\end{array}\right)=\left(\begin{array}{cc}
F_{11} & 0 \\
F_{21} & F_{22}
\end{array}\right)\left(\begin{array}{l}
\delta z_{1} \\
\delta z_{2}
\end{array}\right)
$$

où les éléments de $F_{21}$ sont majorés. La première équation ne dépendant pas de la seconde, on peut conclure à la convergence exponentielle de $\delta z_{1}$ vers zéro si la matrice $F_{11}$ est uniformément définie négative.

Nous poursuivons le raisonnement en considérant $F_{21} \delta z_{1}$ comme une perturbation. Puisque $F_{21}$ est majorée, la convergence exponentielle de $\delta z_{1}$ vers zéro assure la dégradation de la perturbation. Ainsi, si $F_{22}$ est uniformément définie négative alors $\delta z_{2}$ converge exponentiellement vers zéro.

La convergence exponentielle du système dans sa globalité vers une seule et même trajectoire est donc prouvée.

L'étude de la convergence joue un rôle particulièrement important dans l'étude des systèmes non linéaires. Il s'agit d'un problème bien connu dans le cadre de la synthèse de commandes et du diagnostic de système. En effet, garantir la décroissance exponentielle vers zéro des résidus en l'absence de défauts est une propriété importante permettant de faciliter la prise de décision (détection et isolation des défauts). Dans le paragraphe suivant, nous proposons une méthode permettant d'appliquer la théorie de la contraction au diagnostic.

\subsubsection{Etude de la convergence des filtres d'isolation}

L'objectif de ce paragraphe est d'exposer une autre solution pour étudier la convergence que celle utilisant les fonctions de Lyapounov. D'après l'écriture du système (3.67), il est naturel 
de synthétiser dans un premier temps un filtre de même dynamique que le système :

$$
\dot{z}(t)=f(z(t), t)
$$

Nous cherchons à prouver la convergence exponentielle vers zéro de l'erreur d'estimation $e(t)=z(t)-x(t)$ qui constitue un résidu particulier. Nous rappelons que dans ce document les études sont réalisées en l'absence d'incertitudes de modèles. Evidemment, si l'état initial est parfaitement connu alors $e(t)=0, \forall t$. En revanche, dans le cas contraire, il n'y a pas de raison que $e(t)$ tende vers zéro. Nous proposons, à l'aide de la théorie de la contraction, une méthode visant à assurer la convergence exponentielle de $e(t)$ quelles que soient les conditions initiales. En d'autres termes, nous cherchons à montrer que les solutions des équations différentielles (3.67) et $(3.84), x(t)$ et $z(t)$ tendent vers une seule et même trajectoire.

Dans un premier temps, l'équation différentielle du filtre est identique (en terme de dynamique) à celle du système, toute solution de (3.67) est nécessairement solution de l'équation différentielle (3.84). Ainsi si la matrice jacobienne $\frac{\partial f(x(t), t)}{\partial x(t)}$ (ou jacobienne généralisée) est définie uniformément négative alors le système est contractant. Dans ce cas, $x(t)$ et $z(t)$ tendent exponentiellement vers une seule et même trajectoire ce qui implique que $e(t)$ tende exponentiellement vers zéro.

Dans le cas contraire, où la convergence ne peut être prouvée ni par l'étude de la jacobienne ni à l'aide des propriétés de connection précédemment rappelées, il est nécessaire d'adjoindre un retour de sortie au filtre.

Soit le système non linéaire muni de l'équation de sortie suivant :

$$
\left\{\begin{array}{l}
\dot{x}(t)=f(x(t), t) \\
y_{x}(t)=h(x(t))
\end{array}\right.
$$

Nous synthétisons un filtre qui copie la dynamique du système précédent tout en y ajoutant une injection de sortie (ou retour de sortie) :

$$
\left\{\begin{array}{l}
\dot{z}(t)=f(z(t), t)+\Psi^{a}\left(z(t), u(t), y_{x}(t), y_{z}(t)\right) \\
y_{z}(t)=h(z(t))
\end{array}\right.
$$

Ce filtre est contractant s'il satisfait à certaines propriétés. En effet, l'équation du système doit toujours vérifier celle du filtre ainsi :

$$
f(z(t), t)+\left.\Psi^{a}\left(z(t), u(t), y_{x}(t), y_{z}(t)\right)\right|_{z(t)=x(t)}=f(x(t), t)
$$

Une solution relativement simple menant à la vérification de cette égalité est d'imposer :

$$
\Psi^{a}\left(z(t), u(t), y_{x}(t), y_{z}(t)\right)=\Psi^{a}\left(z(t), u(t), y_{x}(t)\right)-\Psi^{a}\left(z(t), u(t), y_{z}(t)\right)
$$


car dans ce cas :

$$
\left.\Psi^{a}\left(z(t), u(t), y_{x}(t)\right)\right|_{z(t)=x(t)}=\left.\Psi^{a}\left(z(t), u(t), y_{z}(t)\right)\right|_{z(t)=x(t)}
$$

et toute trajectoire du système est ainsi une trajectoire du filtre. Si nous prouvons que le filtre et le système tend vers une seule et même trajectoire indépendamment de l'état initial, alors toute estimation du filtre tendra vers l'état réel du système.

En considérant la matrice jacobienne du filtre $\frac{\partial}{\partial z(t)}\left(f(z(t), t)+\Psi^{a}\left(z(t), u(t), y_{x}(t), y_{z}(t)\right)\right)$, l'injection de sortie permet d'ajouter des degrés de liberté. En effet, grâce à la linéarité de l'opérateur dérivée partielle, la matrice jacobienne du filtre se décompose en la somme $\frac{\partial}{\partial z(t)} f(z(t), t)+\frac{\partial}{\partial z(t)} \Psi^{a}\left(z(t), u(t), y_{x}(t), y_{z}(t)\right)$ dont :

- la première partie est fixée par la dynamique du système,

- la deuxième partie, mise à part la contrainte définie par l'équation (3.88), est libre.

Ainsi, à ce stade de l'étude, l'objectif est de prouver qu'à l'aide de l'injection de sortie il est possible :

- soit de se ramener à une forme bien connue de combinaison (parallèle, "feedback" ou hiérarchique),

- soit de prouver d'une manière générale que la matrice jacobienne généralisée est uniformément définie négative.

et dans ce cas il est possible de prouver la convergence de l'erreur d'estimation vers zéro.

Ce type d'étude peut être appliqué au cas des systèmes découplés ayant pour objectif la garantie de l'estimation d'une partie des états malgré la présence de défauts.

\subsubsection{Application de la théorie de la contraction au découplage}

L'objectif de ce paragraphe est d'expliquer dans un premier temps la méthodologie à suivre dans le cadre d'un découplage mono-défaut (section 3.2.1) ([39]). L'extension au cas monofiltre multi-défauts est proposée par la suite.

L'augmentation de l'injection de sortie menant à la convergence exponentielle nécessite des restrictions supplémentaires à celles dues au découplage.

Nous considérons l'écriture d'un système non linéaire dans une base de coordonnées particulière :

$$
\Sigma_{\widetilde{N L}}:\left\{\begin{array}{l}
\dot{\tilde{x}}_{1}(t)=\tilde{f}_{0,1}\left(\tilde{x}_{1}(t), \tilde{x}_{2}(t)\right)+\sum_{i=1}^{m} \tilde{f}_{i, 1}\left(\tilde{x}_{1}(t), \tilde{x}_{2}(t)\right) u_{i}(t)+\tilde{P}_{x}\left(\tilde{x}_{1}(t), \tilde{x}_{2}(t)\right) w(t) \\
\dot{\tilde{x}}_{2}(t)=\tilde{f}_{0,2}\left(\tilde{x}_{1}(t), \tilde{x}_{2}(t)\right)+\sum_{i=1}^{m} \tilde{f}_{i, 2}\left(\tilde{x}_{1}(t), \tilde{x}_{2}(t)\right) u_{i}(t) \\
y_{x}(t)=\tilde{h}\left(\tilde{x}_{1}(t), \tilde{x}_{2}(t)\right)
\end{array}\right.
$$


Nous associons à ce système, le filtre non linéaire synthétisé à l'aide d'une injection de sortie conformément au paragraphe 3.2.1.1 de ce chapitre :

$$
\Sigma_{\widetilde{F N L}}:\left\{\begin{aligned}
\dot{\tilde{z}}_{1}(t) & =\tilde{f}_{0,1}\left(\tilde{z}_{1}(t), \tilde{z}_{2}(t)\right)+\sum_{i=1}^{m} \tilde{f}_{i, 1}\left(\tilde{z}_{1}(t), \tilde{z}_{2}(t)\right) u_{i}(t) \\
\dot{\tilde{z}}_{2}(t) & =\tilde{f}_{0,2}\left(\tilde{z}_{1}(t), \tilde{z}_{2}(t)\right)+\sum_{i=1}^{m} \tilde{f}_{i, 2}\left(\tilde{z}_{1}(t), \tilde{z}_{2}(t)\right) u_{i}(t) \\
& +\tilde{\Psi}\left(\tilde{z}_{2}(t), u(t), y_{x}(t)\right)-\tilde{\Psi}\left(\tilde{z}_{2}(t), u(t), y_{z}(t)\right) \\
y_{z}(t) & =\tilde{h}\left(\tilde{z}_{1}(t), \tilde{z}_{2}(t)\right)
\end{aligned}\right.
$$

En choisissant l'injection de sortie comme nous l'expliquons dans la section précédente, le sous-espace d'état associé à $\tilde{z}_{1}(t)$ est nécessairement invariant à la dynamique du système. Ainsi, l'injection de sortie $\tilde{\Psi}\left(\tilde{z}_{2}(t), u(t), y_{z}(t)\right)$ vérifie l'égalité suivante :

$$
\begin{aligned}
& \tilde{f}_{i, 2}\left(\tilde{z}_{1}(t), \tilde{z}_{2}(t)\right)-\tilde{\Psi}\left(\tilde{z}_{2}(t), u(t), y_{z}(t)\right)=\breve{f}_{i, 2}\left(\tilde{z}_{2}(t)\right) \\
& \text { avec } i=\{0, \cdots, m\}
\end{aligned}
$$

D'après cette définition, le filtre (3.91) s'écrit :

$$
\Sigma_{\widetilde{F N L}}:\left\{\begin{array}{l}
\dot{\tilde{z}}_{1}(t)=\tilde{f}_{0,1}\left(\tilde{z}_{1}(t), \tilde{z}_{2}(t)\right)+\sum_{i=1}^{m} \tilde{f}_{i, 1}\left(\tilde{z}_{1}(t), \tilde{z}_{2}(t)\right) u_{i}(t) \\
\dot{\tilde{z}}_{2}(t)=\breve{f}_{0,2}\left(\tilde{z}_{2}(t)\right)+\sum_{i=1}^{m} \breve{f}_{i, 2}\left(\tilde{z}_{2}(t)\right) u_{i}(t)+\tilde{\Psi}\left(\tilde{z}_{2}(t), u(t), y_{x}(t)\right) \\
y_{z}(t)=\tilde{h}_{(}\left(\tilde{z}_{1}(t), \tilde{z}_{2}(t)\right)
\end{array}\right.
$$

en y incluant la forme de l'injection de sortie augmentée (3.86) $\left(\Psi^{a}\left(z(t), y_{x}(t), y_{z}(t)\right)\right)$, nous écrivons le filtre comme suit :

$$
\Sigma_{\widetilde{F N L}}:\left\{\begin{aligned}
\dot{\tilde{z}}_{1}(t) & =\tilde{f}_{0,1}\left(\tilde{z}_{1}(t), \tilde{z}_{2}(t)\right)+\sum_{i=1}^{m} \tilde{f}_{i, 1}\left(\tilde{z}_{1}(t), \tilde{z}_{2}(t)\right) u_{i}(t)+\tilde{\Psi}_{1}^{a}\left(z(t), u(t), y_{x}(t), y_{z}(t)\right) \\
\dot{\tilde{z}}_{2}(t) & =\breve{f}_{0,2}\left(\tilde{z}_{2}(t)\right)+\sum_{i=1}^{m} \breve{f}_{i, 2}\left(\tilde{z}_{2}(t)\right) u_{i}(t) \\
& +\tilde{\Psi}\left(\tilde{z}_{2}(t), u(t), y_{x}(t)\right)+\tilde{\Psi}_{2}^{a}\left(z(t), u(t), y_{x}(t), y_{z}(t)\right) \\
y_{z}(t) & =\tilde{h}_{(}\left(\tilde{z}_{1}(t), \tilde{z}_{2}(t)\right)
\end{aligned}\right.
$$


Lors de l'analyse de la convergence, il s'avère nécessaire de décomposer les sorties de la manière suivante :

$$
\Sigma_{\widetilde{F N L}}:\left\{\begin{aligned}
\dot{\tilde{z}}_{1}(t) & =\tilde{f}_{0,1}\left(\tilde{z}_{1}(t), \tilde{z}_{2}(t)\right)+\sum_{i=1}^{m} \tilde{f}_{i, 1}\left(\tilde{z}_{1}(t), \tilde{z}_{2}(t)\right) u_{i}(t)+\tilde{\Psi}_{1}^{a}\left(\tilde{z}(t), u(t), y_{x}(t), y_{z}(t)\right) \\
\dot{\tilde{z}}_{2}(t) & =\breve{f}_{0,2}\left(\tilde{z}_{2}(t)\right)+\sum_{i=1}^{m} \breve{f}_{i, 2}\left(\tilde{z}_{2}(t)\right) u_{i}(t) \\
& +\tilde{\Psi}_{(}\left(\tilde{z}_{2}(t), u(t), y_{x}(t)\right)+\tilde{\Psi}_{2}^{a}\left(\tilde{z}(t), u(t), y_{x}(t), y_{z}(t)\right) \\
y_{z 1}(t) & =\breve{h}_{1}\left(\tilde{z}_{1}(t), \tilde{z}_{2}(t)\right) \\
y_{z 2}(t) & =\breve{h}_{2}\left(\tilde{z}_{2}(t)\right)
\end{aligned}\right.
$$

Nous assurons l'égalité parfaite de $y_{z 2}(t)$ et de $y_{x 2}(t)$ en l'absence d'erreur d'initialisation. En revanche, il est évident que toute fonction de $y_{z 1}(t)-y_{x 1}(t)$ est dépendante du défaut $w(t)$. Cependant, nous avons déjà mis en relief une contrainte particulière (3.88) due à la concordance des trajectoires du filtre et du système. Dans le but de ne pas détériorer les caractéristiques liées au découplage du défaut, nous restreignons à nouveau l'injection de sortie augmentée à l'aide des égalités suivantes :

$$
\begin{aligned}
& \tilde{\Psi}_{1}^{a}\left(\tilde{z}(t), u(t), y_{x}(t), y_{z}(t)\right)=\tilde{\Psi}_{1}^{a}\left(\tilde{z}(t), u(t), y_{x 1}(t), y_{x 2}(t), y_{z 1}, y_{z 2}(t)\right) \\
& \tilde{\Psi}_{2}^{a}\left(\tilde{z}(t), u(t), y_{x}(t), y_{z}(t)\right)=\tilde{\Psi}_{1}^{a}\left(\tilde{z}_{2}(t), u(t), y_{x 2}(t), y_{z 2}(t)\right)
\end{aligned}
$$

En considérant ces deux restrictions (l'une due aux notions de trajectoire, l'autre liée au découplage), nous obtenons l'injection de sortie augmentée suivante :

$$
\begin{aligned}
& \tilde{\Psi}_{1}^{a}\left(\tilde{z}(t), u(t), y_{x}(t), y_{z}(t)\right)=\tilde{\Psi}_{1}^{a}\left(\tilde{z}(t), u(t), y_{x 1}(t), y_{x 2}(t)\right)-\tilde{\Psi}_{1}^{a}\left(\tilde{z}(t), u(t), y_{z 1}(t), y_{z 2}(t)\right) \\
& \tilde{\Psi}_{2}^{a}\left(\tilde{z}(t), u(t), y_{x}(t), y_{z}(t)\right)=\tilde{\Psi}_{2}^{a}\left(\tilde{z}_{2}(t), u(t), y_{x 2}(t)\right)-\tilde{\Psi}_{2}^{a}\left(\tilde{z}_{2}(t), u(t), y_{z 2}(t)\right)
\end{aligned}
$$

La matrice jacobienne du filtre, suite à ces égalités, s'écrit comme suit :

$$
\begin{aligned}
& \widetilde{J a c}_{F D}=\frac{\partial \dot{\tilde{z}}(t)}{\partial \tilde{z}(t)}=\left(\begin{array}{cc}
\alpha_{1}+\beta_{1} & \alpha_{2}+\beta_{2} \\
0 & \alpha_{3}+\beta_{3}
\end{array}\right) \\
& =\frac{\partial}{\partial \tilde{z}(t)}\left(\begin{array}{c}
\tilde{f}_{0,1}\left(\tilde{z}_{1}(t), \tilde{z}_{2}(t)\right)+\sum_{i=1}^{m} \tilde{f}_{i, 1}\left(\tilde{z}_{1}(t), \tilde{z}_{2}(t)\right) u_{i}(t)+\tilde{\Psi}_{1}^{a}\left(\tilde{z}(t), u(t), y_{x}(t), y_{z}(t)\right) \\
\breve{f}_{0,2}\left(\tilde{z}_{2}(t)\right)+\sum_{i=1}^{m} \tilde{f}_{i, 2}\left(\tilde{z}_{2}(t)\right) u_{i}(t)+\tilde{\Psi}\left(\tilde{z}_{2}(t), u(t), y_{x}(t)\right)+\tilde{\Psi}_{2}^{a}\left(\tilde{z}(t), u(t), y_{x}(t), y_{z}(t)\right)
\end{array}\right)
\end{aligned}
$$

Ainsi les degrés de liberté, $\beta_{j}, j=\{1,2,3\}$ sont définis par :

$$
\begin{aligned}
& \beta_{1}=\frac{\partial}{\partial \tilde{z}_{1}(t)}\left(\tilde{\Psi}_{1}^{a}\left(\tilde{z}(t), u(t), y_{x}(t), y_{z}(t)\right)\right) \\
& \beta_{2}=\frac{\partial}{\partial \tilde{z}_{2}(t)}\left(\tilde{\Psi}_{1}^{a}\left(\tilde{z}(t), u(t), y_{x}(t), y_{z}(t)\right)\right) \\
& \beta_{3}=\frac{\partial}{\partial \tilde{z}_{2}(t)}\left(\tilde{\Psi}_{2}^{a}\left(\tilde{z}_{2}(t), u(t), y_{x 2}(t), y_{z 2}(t)\right)\right)
\end{aligned}
$$


Au même titre que pour l'étude de l'observabilité dans le cadre des modèles linéaires $\operatorname{span}\left\{d h^{T}\right\}$ joue un rôle très important dans le calcul menant à la convergence du filtre. Les coefficients $\beta_{j}$ sont en effet choisis de manière à rendre, dans la mesure du possible, la matrice $\widetilde{J a c}_{F D}$ uniformément définie négative. Mais comme nous l'avons précisé dans cette section, il est possible de montrer différemment la convergence en considérant les propriétés d'inter-connection.

En supposant qu'à l'aide des degrés de liberté, la convergence du filtre et du système vers une seule et même trajectoire est prouvée, nous assurons la convergence exponentielle de $z(t)$ vers $x(t)$, seulement si le défaut n'est pas présent sur le système $(w(t)=0)$. En revanche, si le défaut est présent, nous assurons tout de même la convergence de la partie découplée $z_{2}(t)$ (i.e. insensible au défaut).

En conclusion, nous insistons sur les contraintes que doit satisfaire l'injection de sortie (définie par le couple d'égalités (3.97)). Dans le cadre d'un découplage de plusieurs défauts à l'aide d'un seul filtre (cas développé dans le paragraphe 3.2.1.2 par exemple), la contrainte liée à la concordance des trajectoires reste identique à précédemment :

$$
\tilde{\Psi}_{a}\left(\tilde{z}(t), u(t), y_{x}(t), y_{z}(t)\right)=\tilde{\Psi}_{a}\left(\tilde{z}(t), u(t), y_{x}(t)\right)-\tilde{\Psi}_{a}\left(\tilde{z}(t), u(t), y_{z}(t)\right)
$$

En revanche, les restrictions liées au découplage sont plus fortes. Nous le montrons par l'écriture du système (3.29) muni cette fois d'une injection de sortie augmentée :

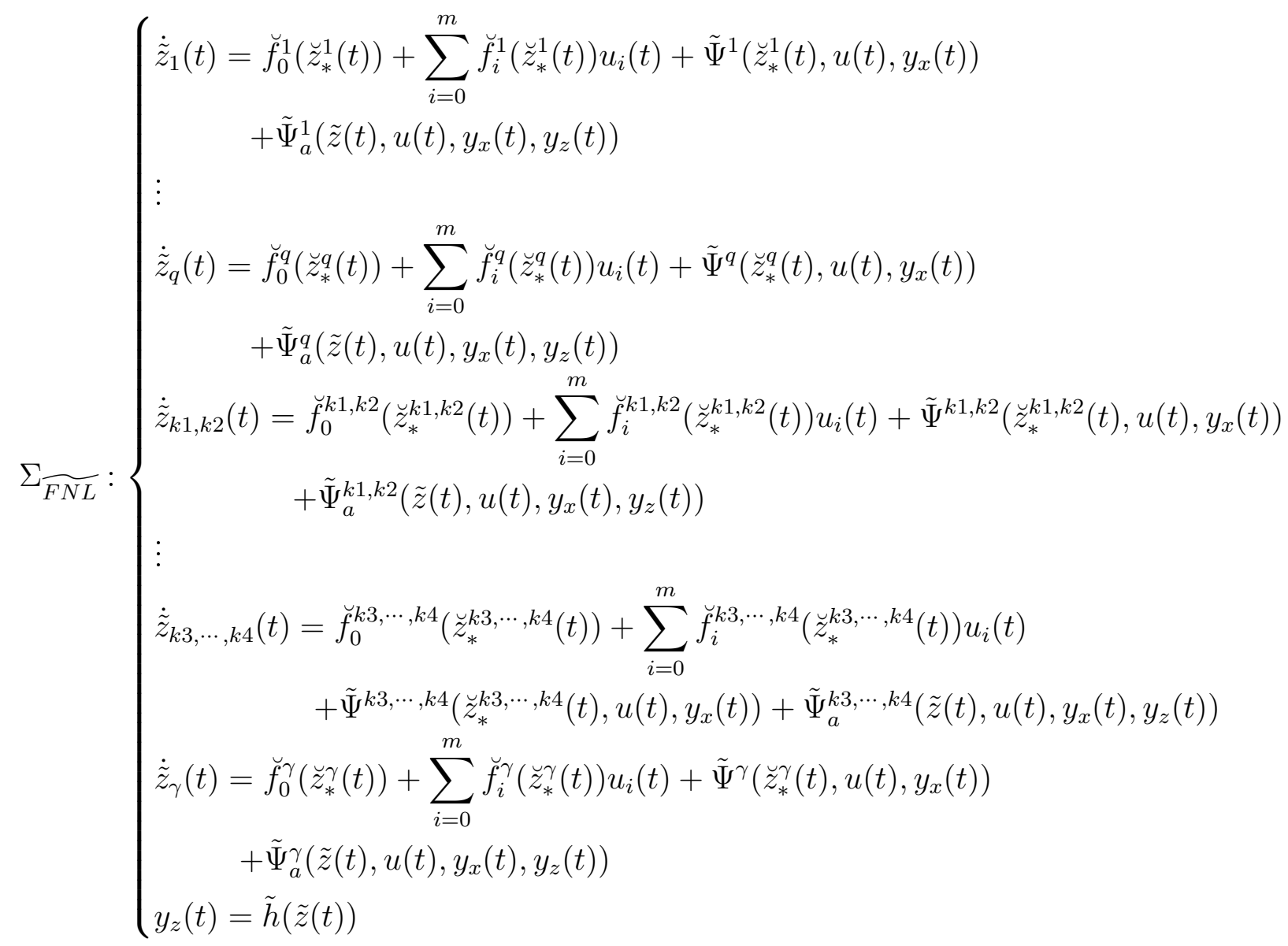


avec

$$
\begin{aligned}
& \left(\begin{array}{lllllll}
\breve{z}_{*}^{1}(t) & \cdots & \breve{z}_{*}^{q}(t) & \breve{z}_{*}^{k 1, k 2}(t) & \cdots & \breve{z}^{k 3, \cdots, k 4}(t) & \breve{z}_{*}^{\gamma}(t)
\end{array}\right)= \\
& \left(\left[\tilde{z}_{1}(t), \tilde{z}_{\gamma}(t)\right] \cdots\left[\tilde{z}_{q}(t), \tilde{z}_{\gamma}(t)\right] \quad\left[\tilde{z}_{k 1}(t), \tilde{z}_{2}(t), \tilde{z}_{k 2, k 3}(t), \tilde{z}_{\gamma}(t)\right]\right. \\
& \left.\cdots\left[\tilde{z}_{k 3}(t), \cdots, \quad \tilde{z}_{k 4}(t), \tilde{z}_{k 3, \cdots, k 4}(t), \tilde{z}_{\gamma}(t)\right]\left[\tilde{z}_{\gamma}(t)\right]\right)
\end{aligned}
$$

En décomposant la sortie comme suit :

$$
\begin{aligned}
& \left(\begin{array}{lllllll}
y_{z}^{1}(t) & \cdots & y_{z}^{q}(t) & y_{z}^{k 1, k 2}(t) & \cdots & y_{z}^{k 3, \cdots, k 4}(t) & y_{z}^{\gamma}(t)
\end{array}\right)^{T}=
\end{aligned}
$$

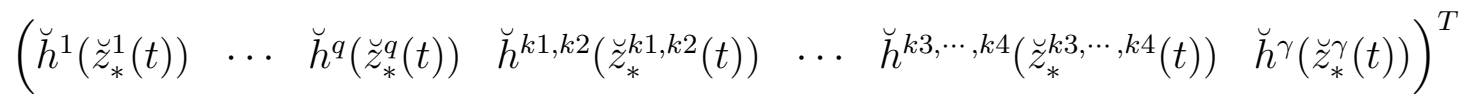

la condition qui permet de conserver le découplage se traduit comme suit :

$$
\begin{aligned}
& \tilde{\Psi}_{a}^{\bullet}\left(\tilde{z}(t), u(t), y_{x}(t), y_{z}(t)\right)=\tilde{\Psi}_{a}^{\bullet}\left(\breve{z}_{*}^{\bullet}(t), u(t), y_{x}^{\bullet}(t)\right)-\tilde{\Psi}_{a}^{\bullet}\left(\breve{z}_{*}^{\bullet}(t), u(t), y_{z}^{\bullet}(t)\right) \\
& \text { avec } \bullet=\left[\begin{array}{lllllll}
{[1]} & \cdots & {[q]} & {[k 1, k 2]} & \cdots & {[k 3, \cdots, k 4]} & {[\gamma]}
\end{array}\right]
\end{aligned}
$$

Satisfaire l'égalité précédente constitue une contrainte relativement forte car toutes les composantes de l'injection de sortie sont concernées.

Pour conclure cette section, nous insistons sur la nécessité d'un compromis entre isoler tous les défauts à l'aide d'un unique filtre et prouver la convergence.

\subsection{Conclusion}

Supposant les conditions d'isolation satisfaites, nous nous attachons, dans ce chapitre, à la synthèse de filtres générateurs de résidus assurant l'isolation des défauts. Les filtres que nous proposons sont synthétisés à l'aide d'injections de sortie de natures différentes (utilisant les signaux de sortie exclusivement et/ou leurs dérivées). Ainsi, nous associons la synthèse d'un filtre à la détermination de son injection de sortie. Se fondant sur les contraintes d'invariance issues de l'analyse structurelle, une méthode de détermination explicite de l'injection de sortie est proposée. Naturellement, nous montrons que ces contraintes sont moins restrictives lorsqu'un banc de filtres est synthétisé. Cependant une évolution de ces techniques consiste à isoler l'ensemble du vecteur défaut à l'aide d'un seul et même filtre. C'est pourquoi, bien que des conditions de construction supplémentaires soient nécessaires à l'isolation des défauts à l'aide d'un seul et même filtre, nous proposons une méthode menant à ce résultat.

Lors de la synthèse de filtres, un problème supplémentaire consiste à prouver la convergence des estimations issues d'un ou plusieurs filtres. L'intérêt de notre proposition est d'assurer, malgré des erreurs d'initialisation, la convergence des estimations vers les états réels du système. 
A cette fin, nous appliquons la théorie de la contraction qui permet de prouver la convergence exponentielle, sous certaines conditions. Nous proposons une extension à cette théorie afin de conserver les propriétés de découplage tout en assurant la convergence des estimations. De plus certains états, même en présence de défauts, sont correctement estimés. 



\section{Chapitre 4}

\section{Application au système des trois cuves}

Dans ce chapitre, nous appliquons l'ensemble des méthodes développées dans les chapitres précédents au système des trois cuves (figure 4.1). Nous avons choisi d'étudier ce système car nous en connaissons relativement bien la description mathématique. Ainsi nous illustrons l'ensemble de nos résultats à l'aide d'un simulateur de ce système ([42] et [60]).

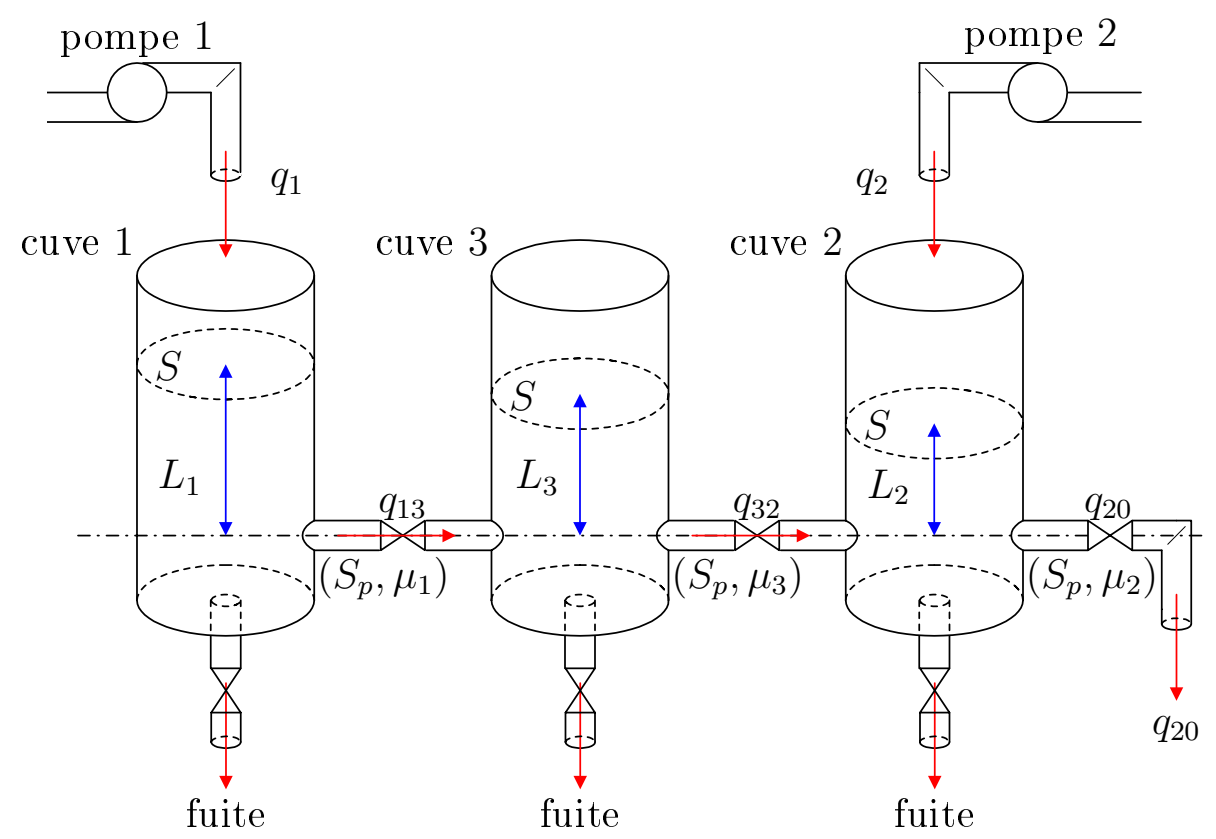

FIG. 4.1: Schématisation du système des 3 cuves

\subsection{Description du système}

Le benchmark considéré se compose de trois cuves cylindriques de section identique $S$. Les cuves sont reliées par deux tuyaux cylindriques de section $S_{p}$ dont le coefficient de viscosité est $\mu_{1}=\mu_{3}$. La sortie du système est située au niveau de la cuve 2, elle est aussi caractérisée par une section $S_{p}$ et un coefficient de viscosité $\mu_{2}$. Deux pompes commandées par des moteurs à courant continu alimentent les cuves 1 et 2 avec les débits $q_{1}(t)$ et $q_{2}(t)$. Les trois 
cuves sont équipées de capteurs de pression pour mesurer le niveau de liquide $\left(L_{1}(t), L_{2}(t)\right.$ et $\left.L_{3}(t)\right)$.

\subsection{Modèle mathématique du système}

En écrivant les équations de la conservation du volume de liquide, nous obtenons :

$$
\Sigma_{N L}:\left\{\begin{array}{l}
S \frac{d L_{1}(t)}{d t}=q_{1}(t)-q_{13}(t) \\
S \frac{d L_{2}(t)}{d t}=q_{2}(t)+q_{32}(t)-q_{20}(t) \\
S \frac{d L_{3}(t)}{d t}=q_{13}(t)-q_{32}(t)
\end{array}\right.
$$

où $q_{i j}(t)$ représente le débit de liquide de la cuve $i$ vers la cuve $j(i, j=1,2,3 \forall i \neq j)$ qui peut être exprimé en utilisant la loi de Torricelli par :

$$
q_{i j}(t)=\mu_{i} . S_{p} \cdot \operatorname{sign}\left(L_{i}(t)-L_{j}(t)\right) \cdot \sqrt{2 g\left|L_{i}(t)-L_{j}(t)\right|}
$$

et $q_{20}(t)$ représente le débit de sortie, avec :

$$
q_{20}(t)=\mu_{2} \cdot S_{p} \cdot \sqrt{2 g L_{2}(t)}
$$

Sans restreindre notre étude, nous considérons le système tel que les niveaux vérifient les inégalités suivantes: $L_{1}(t)>L_{3}(t)>L_{2}(t)$. En d'autres termes, nous considérons un sens particulier des débits inter-cuves $\left(q_{i j}(t)\right)$. Avec ces équations, nous supposons que le système des 3 cuves est parfaitement décrit à l'aide du modèle non linéaire affine en la commande défini équation (1.5) :

$$
\Sigma_{N L}:\left\{\begin{array}{l}
\dot{x}_{1}(t)=-2 C_{1} \sqrt{x_{1}(t)-x_{3}(t)}+u_{1}(t) / S \\
\dot{x}_{2}(t)=2 C_{3} \sqrt{x_{3}(t)-x_{2}(t)}-2 C_{2} \sqrt{x_{2}(t)}+u_{2}(t) / S \\
\dot{x}_{3}(t)=2 C_{1} \sqrt{x_{1}(t)-x_{3}(t)}-2 C_{3} \sqrt{x_{3}(t)-x_{2}(t)} \\
y_{x 1}(t)=x_{1}(t) \\
y_{x 2}(t)=x_{2}(t) \\
y_{x 3}(t)=x_{3}(t)
\end{array}\right.
$$

avec $x_{i}(t)$ le niveau de liquide dans la cuve $i$ et $C_{i}=(1 / 2) \cdot(1 / S) \cdot \mu_{i} \cdot S_{p} \cdot \sqrt{2 g}$. Les deux signaux de commande $u_{1}(t), u_{2}(t)$ sont respectivement les deux débits d'entrée $q_{1}(t)$ et $q_{2}(t)$. De plus, puisque les 3 niveaux sont mesurés, il est trivial que $\left(\gamma_{o b s}\right)^{\perp}$ et $\left(\gamma_{y}\right)^{\perp}$ sont réduits à l'ensemble $\{0\}$. 


\subsection{Diagnostic du système}

Nous récrivons le système en faisant apparaître les différents défauts que nous étudions dans ce chapitre :

$$
\Sigma_{N L}:\left\{\begin{array}{l}
\dot{x}_{1}(t)=-2 C_{1} \sqrt{x_{1}(t)-x_{3}(t)}+\left(u_{1}(t)+w_{a 1}(t)\right) / S \\
\dot{x}_{2}(t)=2 C_{3} \sqrt{x_{3}(t)-x_{2}(t)}-2 C_{2} \sqrt{x_{2}(t)}+\left(u_{2}(t)+w_{a 2}(t)\right) / S \\
\dot{x}_{3}(t)=2 C_{1} \sqrt{x_{1}(t)-x_{3}(t)}-2 C_{3} \sqrt{x_{3}(t)-x_{2}(t)} \\
y_{x 1}(t)=x_{1}(t)+w_{c 1}(t) \\
y_{x 2}(t)=x_{2}(t)+w_{c 2}(t) \\
y_{x 3}(t)=x_{3}(t)+w_{c 3}(t)
\end{array}\right.
$$

avec $w_{a i}(t)$ le défaut associé à l'actionneur $i(i=\{1,2\})$ et $w_{c j}(t)$ le défaut associé au capteur $j(j=\{1,2,3\})$.

Nous considérons exclusivement les défauts de type actionneur ou capteur.

\subsubsection{Défauts actionneur}

Dans cette section, seuls les défauts actionneur notés $P_{1} w_{a 1}(t)=\left(\begin{array}{lll}1 / S & 0 & 0\end{array}\right)^{T} w_{a 1}(t)$ et $P_{2} w_{a 2}(t)=\left(\begin{array}{lll}0 & 1 / S & 0\end{array}\right)^{T} w_{a 2}(t)$ sont considérés $\left(i . e . w_{c 1}(t)=w_{c 2}(t)=w_{c 3}(t)=0, \forall t\right)$. Nous calculons les différentes distributions définies dans les chapitres précédents :

$$
\begin{array}{ll}
C_{*}^{P_{1}}=\operatorname{span}\left\{\left(\begin{array}{l}
1 \\
0 \\
0
\end{array}\right),\left(\begin{array}{l}
0 \\
1 \\
0
\end{array}\right),\left(\begin{array}{l}
0 \\
0 \\
1
\end{array}\right)\right\} & C_{*}^{P_{2}}=\operatorname{span}\left\{\left(\begin{array}{l}
1 \\
0 \\
0
\end{array}\right),\left(\begin{array}{l}
0 \\
1 \\
0
\end{array}\right),\left(\begin{array}{l}
0 \\
0 \\
1
\end{array}\right)\right\} \\
\left(C_{*}^{P_{1}}\right)^{\perp}=\operatorname{span}\{0\} & \left(C_{*}^{P_{2}}\right)^{\perp}=\operatorname{span}\{0\} \\
S_{*}^{P_{1}}=\operatorname{span}\left\{\left(\begin{array}{l}
1 \\
0 \\
0
\end{array}\right)\right\} & S_{*}^{P_{2}}=\operatorname{span}\left\{\left(\begin{array}{l}
0 \\
1 \\
0
\end{array}\right)\right\} \\
\left(S_{*}^{P_{1}}\right)^{\perp}=\operatorname{span}\left\{\left(\begin{array}{l}
0 \\
1 \\
0
\end{array}\right),\left(\begin{array}{l}
0 \\
0 \\
1
\end{array}\right)\right\} \quad\left(S_{*}^{P_{2}}\right)^{\perp}=\operatorname{span}\left\{\left(\begin{array}{l}
1 \\
0 \\
0
\end{array}\right),\left(\begin{array}{l}
0 \\
0 \\
1
\end{array}\right)\right\} \\
S_{m *}^{P_{1}}=\operatorname{span}\{0\} \\
\left(S_{m *}^{P_{1}}\right)^{\perp}=\operatorname{span}\left\{\left(\begin{array}{l}
1 \\
0 \\
0
\end{array}\right),\left(\begin{array}{l}
0 \\
1 \\
0
\end{array}\right),\left(\begin{array}{l}
0 \\
0 \\
1
\end{array}\right)\right\} \quad\left(S_{m *}^{P_{2}}\right)^{\perp}=\operatorname{span}\left\{\left(\begin{array}{l}
1 \\
0 \\
0
\end{array}\right),\left(\begin{array}{l}
0 \\
1 \\
0
\end{array}\right),\left(\begin{array}{l}
0 \\
0 \\
1
\end{array}\right)\right\}
\end{array}
$$

Remarquons la nullité de $S_{m *}^{P_{1}}\left(\operatorname{resp} . S_{m *}^{P_{2}}\right)$ car il est possible d'exprimer le défaut $w_{a 1}(t)$ (resp. $\left.w_{a 2}(t)\right)$ à partir d'une combinaison des signaux connus. En effet, si nous considérons 
les dérivées des sorties alors $w_{a 1}(t)=S\left(\dot{y}_{x 1}(t)+2 C_{1} \sqrt{y_{x 1}(t)-y_{x 3}(t)}-u_{1}(t) / S\right)$ $\left(\operatorname{resp} . w_{a 2}(t)=S\left(\dot{y}_{x 2}(t)-2 C_{3} \sqrt{y_{x 3}(t)-y_{x 2}(t)}+2 C_{2} \sqrt{y_{x 2}(t)}-u_{2}(t) / S\right)\right)$.

Suite à ces premiers calculs, nous pouvons d'ores et déjà ajouter que les deux défauts sont détectables car :

- le sous-espace insensible au défaut $w_{a 1}(t)$ (resp. $\left.w_{a 2}(t)\right)$ sans injection de sortie est réduit à l'espace nul $\left(C_{*}^{P_{1}}\right)^{\perp}=\{0\}$ (resp. $\left.\left(C_{*}^{P_{2}}\right)^{\perp}=\{0\}\right)$,

- l'espace observable est non nul.

Ainsi, la non-inclusion $\gamma_{o b s} \nsubseteq\left(C_{*}^{P_{1}}\right)^{\perp}$ (resp. $\left.\gamma_{o b s} \nsubseteq\left(C_{*}^{P_{2}}\right)^{\perp}\right)$ est satisfaite et est synonyme de détectabilité ( $c f .(2.23))$.

Dans le troisième chapitre, plusieurs cas (mono-filtre et multi-filtres) ont été étudiés. Pour la synthèse de chaque filtre, une méthode de détermination de l'injection de sortie est proposée. Appliquons ces méthodes au cas des défauts d'actionneur.

\subsubsection{Isolation à l'aide de plusieurs filtres}

Dans cette section, nous développons deux méthodes ayant pour point commun la synthèse de $q$ filtres (ici $q=2$ ). Chaque filtre assure l'isolation d'un défaut particulier. Les deux méthodes exposées se distinguent au travers de la distribution employée $S_{*}^{P_{j}}$ ou $S_{m *}^{P_{j}}$.

\section{Méthode se fondant sur $S_{*}^{P_{j}}$}

Afin de vérifier que les conditions d'isolation sont satisfaites, une analyse structurelle est réalisée.

Puisque $\left(S_{*}^{P_{2}}\right)^{\perp} \nsubseteq\left(S_{*}^{P_{1}}\right)^{\perp}$, il existe au moins une distribution sensible à $w_{1}(t)$ et insensible à $w_{2}(t)$. De plus, il existe une distribution $y$-reconstructible parmi les distributions vérifiant la non inclusion précédente ((1 000$)^{T}$ par exemple), donc $\left(S_{*}^{P_{2}}\right)^{\perp} \cap \gamma_{y} \nsubseteq\left(S_{*}^{P_{1}}\right)^{\perp}$ et $\mathcal{A}_{m}^{P_{1}}=\left(\begin{array}{ll}1 & 0\end{array}\right)$. Nous reproduisons ce raisonnement pour le défaut $w_{2}(t)$. Avec $\left(S_{*}^{P_{1}}\right)^{\perp} \cap \gamma_{y} \nsubseteq\left(S_{*}^{P_{2}}\right)^{\perp}$, nous obtenons l'expression de $\mathcal{A}_{m}^{P_{2}}=\left(\begin{array}{ll}0 & 1\end{array}\right)$. Cette non inclusion est par exemple vérifiée par la distribution $\left(\begin{array}{lll}0 & 1 & 0\end{array}\right)^{T}$ qui est $y$-reconstructible.

Suite à ce raisonnement, la matrice $\mathcal{A}_{m}$ décrivant les différentes sensibilités s'écrit comme suit :

$$
\mathcal{A}_{m}=\left(\begin{array}{ll}
1 & 0 \\
0 & 1
\end{array}\right)
$$

et le théorème 2.12 est donc satisfait. De plus, la matrice $\mathcal{A}_{m}$ est diagonale ce qui signifie qu'il existe une solution au F.P.R.G.

Les filtres suivants sont synthétisés : 
- filtre isolateur du défaut $w_{a 2}(t)$ :

$$
\Sigma_{F \widetilde{N L w_{a 1}}}:\left\{\begin{aligned}
\dot{\tilde{z}}_{1}(t) & =-2 C_{1} \sqrt{\tilde{z}_{1}(t)-\tilde{z}_{22}(t)}+u_{1}(t) / S \\
\dot{\tilde{z}}_{2}(t) & =\left(\begin{array}{c}
\dot{\tilde{z}}_{21}(t) \\
\dot{\tilde{z}}_{22}(t)
\end{array}\right) \\
& =\left(\begin{array}{c}
2 C_{3} \sqrt{\tilde{z}_{22}(t)-\tilde{z}_{21}(t)}-2 C_{2} \sqrt{\tilde{z}_{21}(t)}+u_{2}(t) / S \\
2 C_{1} \sqrt{\tilde{z}_{1}(t)-\tilde{z}_{22}(t)}-2 C_{3} \sqrt{\tilde{z}_{22}(t)-\tilde{z}_{21}(t)}
\end{array}\right) \\
& +\tilde{\Psi}\left(\tilde{z}_{2}(t), y_{x}(t)\right)-\tilde{\Psi}\left(\tilde{z}_{2}(t), y_{z}(t)\right) \\
y_{z 1}(t) & =\tilde{z}_{1}(t) \\
y_{z 2}(t) & =\tilde{z}_{21}(t) \\
y_{z 3}(t) & =\tilde{z}_{22}(t)
\end{aligned}\right.
$$

Puisque la présence de l'état $\tilde{z}_{1}(t)$ est nuisible dans l'équation différentielle régissant $\tilde{z}_{2}(t)$, nous cherchons une injection de sortie qui neutralise son effet. En posant $\tilde{\Psi}\left(\tilde{z}_{2}(t), y_{\bullet}(t)\right)=$ $\left(\begin{array}{ll}0 & 2 C_{1} \sqrt{y_{\bullet 1}(t)-z_{22}(t)}\end{array}\right)^{T}$, nous déterminons une solution qui conduit à l'écriture suivante du filtre dans sa base d'origine :

$$
\Sigma_{F N L w_{a 1}}:\left\{\begin{array}{l}
\dot{z}_{1}(t)=-2 C_{1} \sqrt{z_{1}(t)-z_{3}(t)}+u_{1}(t) / S \\
\dot{z}_{2}(t)=2 C_{3} \sqrt{z_{3}(t)-z_{2}(t)}-2 C_{2} \sqrt{z_{2}(t)}+u_{2}(t) / S \\
\dot{z}_{3}(t)=2 C_{1} \sqrt{y_{x 1}(t)-z_{3}(t)}-2 C_{3} \sqrt{z_{3}(t)-z_{2}(t)} \\
y_{z 1}(t)=z_{1}(t) \\
y_{z 2}(t)=z_{2}(t) \\
y_{z 3}(t)=z_{3}(t)
\end{array}\right.
$$

Ainsi les états $x_{2}(t)$ et $x_{3}(t)$ sont parfaitement estimés par $z_{2}(t)$ et $z_{3}(t) \forall w_{a 1}(t)$. En revanche, en présence du défaut $w_{a 2}(t)$, l'état $x_{2}(t)$ n'est pas identique à $z_{2}(t)$.

- filtre isolateur du défaut $w_{a 1}(t)$ :

Avec un raisonnement similaire, nous déterminons le filtre suivant :

$$
\Sigma_{F N L w_{a 2}}:\left\{\begin{array}{l}
\dot{z}_{1}(t)=-2 C_{1} \sqrt{z_{1}(t)-z_{3}(t)}+u_{1}(t) / S \\
\dot{z}_{2}(t)=2 C_{3} \sqrt{z_{3}(t)-z_{2}(t)}-2 C_{2} \sqrt{z_{2}(t)}+u_{2}(t) / S \\
\dot{z}_{3}(t)=2 C_{1} \sqrt{z_{1}(t)-z_{3}(t)}-2 C_{3} \sqrt{z_{3}(t)-y_{x 2}(t)} \\
y_{z 1}(t)=z_{1}(t) \\
y_{z 2}(t)=z_{2}(t) \\
y_{z 3}(t)=z_{3}(t)
\end{array}\right.
$$

Ainsi, les états $x_{1}(t)$ et $x_{3}(t)$ sont parfaitement estimés par $z_{1}(t)$ et $z_{3}(t) \forall w_{a 2}(t)$. En revanche, en présence du défaut $w_{a 1}(t)$, l'état $x_{1}(t)$ n'est pas identique à $z_{1}(t)$. 
Il est alors trivial que l'étude des résidus formés par :

- la différence $y_{z 1}(t)-y_{x 1}(t)$ du filtre (4.10) permet d'isoler le défaut $w_{a 1}(t)$,

- la différence $y_{z 2}(t)-y_{x 2}(t)$ du filtre (4.9) permet d'isoler le défaut $w_{a 2}(t)$.

De plus, puisque la matrice $\mathcal{A}_{m}$ est diagonale, les défauts sont isolables même s'ils apparaissent simultanément.

\section{Méthode se fondant sur $S_{m *}^{P_{j}}$}

Nous rappelons que pour isoler le défaut $w_{j}(t)$, l'étape d'estimation du défaut (découplage total) doit être inhibée lors du calcul de la distribution $S_{m *}^{P_{j}}$. Ainsi, dans ce cas concret il est facile de justifier l'utilisation des distributions $S_{*}^{P_{j}}$ au détriment de $S_{m *}^{P_{j}}$. Suite au calcul de $S_{m *}^{P_{j}}=\{0\}$ avec $j=\{1,2\}$, il n'est pas possible d'isoler les défauts. En revanche, si nous inhibons l'étape d'estimation des défauts alors $S_{m *}^{P_{1}}=S_{*}^{P_{1}}$ et $S_{m *}^{P_{2}}=S_{*}^{P_{2}}$ d'où leur utilisation. Par la suite, nous considérons les deux distributions suivantes en vis à vis :

- la distribution $S_{*}^{P_{1}}$ est considérée pour isoler le défaut $w_{a 1}(t)$. La deuxième distribution est $S_{m *}^{P_{2}}$ est également utilisée puisque nous cherchons le plus grand sous-espace d'état insensible au défaut $w_{a 2}(t)$,

- les distributions $S_{*}^{P_{2}}$ et $S_{m *}^{P_{1}}$ sont considérées pour isoler le défaut $w_{a 2}(t)$ pour les même raisons que précédemment.

La condition d'isolation (théorème 2.12) est satisfaite car $\left(S_{m *}^{P_{1}}\right)^{\perp} \nsubseteq\left(S_{*}^{P_{2}}\right)^{\perp}$ et $\left(S_{m *}^{P_{2}}\right)^{\perp} \nsubseteq$ $\left(S_{*}^{P_{1}}\right)^{\perp}$.

D'après l'équation $w_{a 1}(t)=S\left(\dot{y}_{x 1}(t)+2 C_{1} \sqrt{y_{x 1}(t)-y_{x 3}(t)}-u_{1}(t) / S\right)$

$\left(\right.$ resp. $\left.w_{a 2}(t)=S\left(\dot{y}_{x 2}(t)-2 C_{3} \sqrt{y_{x 3}(t)-y_{x 2}(t)}+2 C_{2} \sqrt{y_{x 2}(t)}-u_{2}(t) / S\right)\right)$,

l'injection de sortie se déduit facilement car elle estime le défaut $\Psi_{d t}\left(\Delta_{x}\right)=S\left(\dot{y}_{x 1}(t)+2 C_{1} \sqrt{y_{x 1}(t)-y_{x 3}(t)}-u_{1}(t) / S\right)$

$\left(\right.$ resp. $\left.\Psi_{d t}\left(\Delta_{x}\right)=S\left(\dot{y}_{x 2}(t)-2 C_{3} \sqrt{y_{x 3}(t)-y_{x 2}(t)}+2 C_{2} \sqrt{y_{x 2}(t)}-u_{2}(t) / S\right)\right)$.

Les filtres s'écrivent alors comme suit :

- pour l'isolation du défaut $w_{a 2}(t)$ :

$$
\Sigma_{F N L w_{a 1}}: \begin{cases}\dot{z}_{1}(t) & =-2 C_{1} \sqrt{z_{1}(t)-z_{3}(t)}+u_{1}(t) / S \\ & +S\left(\dot{y}_{x 1}(t)-u_{1}(t) / S+C_{1} \sqrt{y_{x 1}(t)-y_{x 3}(t)}\right) \\ \dot{z}_{2}(t) & =2 C_{3} \sqrt{z_{3}(t)-z_{2}(t)}-2 C_{2} \sqrt{z_{2}(t)}+u_{2}(t) / S \\ \dot{z}_{3}(t) & =2 C_{1} \sqrt{z_{1}(t)-z_{3}(t)}-2 C_{3} \sqrt{z_{3}(t)-z_{2}(t)} \\ y_{z 1}(t) & =z_{1}(t) \\ y_{z 2}(t) & =z_{2}(t) \\ y_{z 3}(t) & =z_{3}(t)\end{cases}
$$


Ainsi, les états $x_{1}(t), x_{2}(t)$ et $x_{3}(t)$ sont parfaitement estimés par $z_{1}(t), z_{2}(t)$ et $z_{3}(t)$ $\forall w_{a 1}(t)$. En revanche, en présence du défaut $w_{a 2}(t)$, l'état $x_{2}(t)$ n'est pas identique à $z_{2}(t)$.

- pour l'isolation du défaut $w_{a 1}(t)$ :

$$
\Sigma_{F N L w_{a 2}}: \begin{cases}\dot{z}_{1}(t) & =-2 C_{1} \sqrt{z_{1}(t)-z_{3}(t)}+u_{1}(t) / S \\ \dot{z}_{2}(t) & =2 C_{3} \sqrt{z_{3}(t)-z_{2}(t)}-2 C_{2} \sqrt{z_{2}(t)}+u_{2}(t) / S \\ & +S\left(\dot{y}_{x 2}(t)-2 C_{3} \sqrt{y_{x 3}(t)-y_{x 2}(t)}+2 C_{2} \sqrt{y_{x 2}(t)}-u_{2}(t) / S\right) \\ \dot{z}_{3}(t) & =2 C_{1} \sqrt{z_{1}(t)-z_{3}(t)}-2 C_{3} \sqrt{z_{3}(t)-z_{2}(t)} \\ y_{z 1}(t) & =z_{1}(t) \\ y_{z 2}(t) & =z_{2}(t) \\ y_{z 3}(t) & =z_{3}(t)\end{cases}
$$

Ainsi, les états $x_{1}(t), x_{2}(t)$ et $x_{3}(t)$ sont parfaitement estimés par $z_{1}(t), z_{2}(t)$ et $z_{3}(t)$ $\forall w_{a 2}(t)$. En revanche, en présence du défaut $w_{a 1}(t)$, l'état $x_{1}(t)$ n'est pas identique à $z_{1}(t)$.

Si nous comparons ces résultats avec ceux obtenus lors de l'étude précédente, la différence est significative :

- dans le premier cas (méthode se fondant sur $S_{*}^{P_{j}}$ ), le sous-espace d'état insensible à $w_{a 1}(t)$ (resp. $\left.w_{a 2}(t)\right)$ est de dimension deux pour le filtre (4.9) (resp. (4.10)),

- dans le deuxième cas (méthode se fondant sur $S_{m *}^{P_{j}}$ ), le sous-espace est de dimension trois pour le filtre (4.11) (resp. (4.12)).

En synthétisant les mêmes résidus que précédemment, les deux défauts actionneur sont isolables même s'ils apparaissent simultanément.

Nous avons également présenté, dans le chapitre précédent, des méthodes permettant l'isolation de plusieurs défauts à l'aide d'un seul et même filtre (paragraphe 3.2.1.2). Nous nous proposons de les appliquer à ce système particulier.

\subsubsection{Isolation à l'aide d'un filtre unique}

L'hypothèse de décomposition en somme directe (3.20) est nécessaire à la synthèse d'un unique filtre. Avec $S_{*}^{P_{1}}=\phi^{P_{1}}$ (car il n'y a aucune partie commune avec d'autres distributions, i.e. $\left.\phi^{P_{1}, P_{2}}=\{0\}\right)$ et $S_{*}^{P_{2}}=\phi^{P_{2}}$, cette hypothèse est satisfaite.

A l'aide de ces calculs, nous définissons un difféomorphisme $\tilde{x}(t)=\Phi(x(t))$ de la forme suivante :

$$
\frac{\partial \Phi(x(t))}{\partial x(t)}=\left(\begin{array}{lll}
\phi^{P_{1}} & \phi^{P_{2}} & \gamma
\end{array}\right)^{T}
$$

où $\gamma$ est orthogonal à $\phi^{P_{1}}$ et $\phi^{P_{2}}$, et rend le difféomorphisme intégrable. Nous choisissons $\gamma=\left(\begin{array}{lll}0 & 0 & 1\end{array}\right)^{T}$. 
Dans ce système de coordonnées, le filtre s'écrit comme suit :

$$
\Sigma_{F N w_{a}}:\left\{\begin{aligned}
\dot{\tilde{z}}_{1}(t) & =-2 C_{1} \sqrt{\tilde{z}_{1}(t)-\tilde{z}_{\gamma}(t)}+u_{1}(t) / S \\
& +\tilde{\Psi}^{1}\left(\tilde{z}(t), y_{x}(t)\right)-\tilde{\Psi}^{1}\left(\tilde{z}(t), y_{z}(t)\right) \\
\dot{\tilde{z}}_{2}(t) & =2 C_{3} \sqrt{\tilde{z}_{\gamma}(t)-\tilde{z}_{2}(t)}-2 C_{2} \sqrt{\tilde{z}_{2}(t)}+u_{2}(t) / S \\
& +\tilde{\Psi}^{2}\left(\tilde{z}(t), y_{x}(t)\right)-\tilde{\Psi}^{2}\left(\tilde{z}(t), y_{z}(t)\right) \\
\dot{\tilde{z}}_{\gamma}(t) & =2 C_{1} \sqrt{\tilde{z}_{1}(t)-\tilde{z}_{\gamma}(t)}-2 C_{3} \sqrt{\tilde{z}_{\gamma}(t)-\tilde{z}_{2}(t)} \\
& +\tilde{\Psi}^{\gamma}\left(\tilde{z}_{\gamma}(t), y_{x}(t)\right)-\tilde{\Psi}^{\gamma}\left(\tilde{z}_{\gamma}(t), y_{z}(t)\right) \\
y_{z 1}(t) & =\tilde{z}_{1}(t) \\
y_{z 2}(t) & =\tilde{z}_{2}(t) \\
y_{z 3}(t) & =\tilde{z}_{\gamma}(t)
\end{aligned}\right.
$$

Nous remarquons la présence des deux états $\tilde{z}_{1}(t)$ et $\tilde{z}_{2}(t)$ dans l'équation $\dot{\tilde{z}}_{\gamma}(t)$. Cependant à $\tilde{z}_{\gamma}(t)$ nous associons le sous-espace d'état insensible à tous les défauts. A l'aide de l'injection de sortie, nous cherchons donc à exprimer les états $\tilde{z}_{1}(t)$ et $\tilde{z}_{2}(t)$ respectivement sensibles aux défauts $w_{a 1}(t)$ et $w_{a 2}(t)$. Poser $\tilde{\Psi}^{\gamma}\left(z_{\gamma}(t), y_{\bullet}(t)\right)=2 C_{1} \sqrt{y_{\bullet 1}(t)-\tilde{z}_{\gamma}(t)}-2 C_{3} \sqrt{\tilde{z}_{\gamma}(t)-\tilde{y}_{\bullet 2}(t)}$ et $\tilde{\Psi}^{1}\left(\tilde{z}(t), y_{\bullet}(t)\right)=\tilde{\Psi}^{2}\left(\tilde{z}(t), y_{\bullet}(t)\right)=0$ est une solution et le filtre s'écrit alors :

$$
\Sigma_{F N L w_{a}}:\left\{\begin{array}{l}
\dot{z}_{1}(t)=-2 C_{1} \sqrt{z_{1}(t)-z_{3}(t)}+u_{1}(t) / S \\
\dot{z}_{2}(t)=2 C_{3} \sqrt{z_{3}(t)-z_{2}(t)}-2 C_{2} \sqrt{z_{2}(t)}+u_{2}(t) / S \\
\dot{z}_{3}(t)=2 C_{1} \sqrt{y_{x 1}(t)-z_{3}(t)}-2 C_{3} \sqrt{z_{3}(t)-y_{x 2}(t)} \\
y_{z 1}(t)=z_{1}(t) \\
y_{z 2}(t)=z_{2}(t) \\
y_{z 3}(t)=z_{3}(t)
\end{array}\right.
$$

avec l'égalité suivante $\left(\begin{array}{llll}\tilde{z}_{1}(t) & \tilde{z}_{2}(t) & \tilde{z}_{\gamma}(t)\end{array}\right)^{T}=\left(\begin{array}{lll}z_{1}(t) & z_{2}(t) & z_{3}(t)\end{array}\right)^{T}$.

Si nous synthétisons le vecteur résidu $\left(y_{z 1}(t)-y_{x 1}(t) \quad y_{z 2}(t)-y_{x 2}(t)\right)^{T}$, chacune des composantes n'est sensible qu'à un seul défaut. Ainsi leur isolation est possible à l'aide d'un unique filtre.

Dans la section suivante, nous nous intéressons aux défauts capteur, en supposant l'absence de défauts actionneur. 


\subsubsection{Défauts capteur}

Une augmentation d'état permet d'écrire tous les défauts capteur comme des défauts actionneur :

$$
\Sigma_{N L}:\left\{\begin{array}{l}
\dot{x}_{1}(t)=-2 C_{1} \sqrt{x_{1}(t)-x_{3}(t)}+u_{1}(t) / S \\
\dot{x}_{2}(t)=2 C_{3} \sqrt{x_{3}(t)-x_{2}(t)}-2 C_{2} \sqrt{x_{2}(t)}+u_{2}(t) / S \\
\dot{x}_{3}(t)=2 C_{1} \sqrt{x_{1}(t)-x_{3}(t)}-2 C_{3} \sqrt{x_{3}(t)-x_{2}(t)} \\
\dot{x}_{4}(t)=-\Gamma_{1}\left(x_{4}(t)-w_{c 1}(t)\right) \\
\dot{x}_{5}(t)=-\Gamma_{2}\left(x_{5}(t)-w_{c 2}(t)\right) \\
\dot{x}_{6}(t)=-\Gamma_{3}\left(x_{6}(t)-w_{c 3}(t)\right) \\
y_{x 1}(t)=x_{1}(t)+x_{4}(t) \\
y_{x 2}(t)=x_{2}(t)+x_{5}(t) \\
y_{x 3}(t)=x_{3}(t)+x_{6}(t)
\end{array}\right.
$$

En imposant une dynamique aux défauts $x_{i+3}(t)=\frac{\Gamma_{i}}{s+\Gamma_{i}} w_{c i}(t)$ (qui doit être plus rapide que la dynamique du système), l'écriture précédente est évidente. Ainsi, les distributions

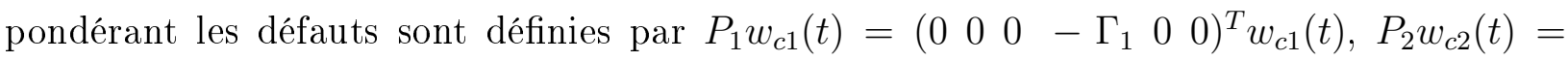
$\left(\begin{array}{lllllll}0 & 0 & 0 & 0 & - & \Gamma_{2} & 0\end{array}\right)^{T} w_{c 2}(t)$ et $P_{3} w_{c 3}(t)=\left(\begin{array}{lllllll}0 & 0 & 0 & 0 & 0 & - & \Gamma_{3}\end{array}\right)^{T} w_{c 3}(t)$.

Le calcul des différentes distributions nécessaires à la synthèse des filtres conduit aux résultats suivants :

$$
\begin{aligned}
& C_{*}^{P_{1}}=\operatorname{span}\left\{\left(\begin{array}{l}
0 \\
0 \\
0 \\
1 \\
0 \\
0
\end{array}\right)\right\} C_{*}^{P_{2}}=\operatorname{span}\left\{\left(\begin{array}{l}
0 \\
0 \\
0 \\
0 \\
1 \\
0
\end{array}\right)\right\} C_{*}^{P_{3}}=\operatorname{span}\left\{\left(\begin{array}{l}
0 \\
0 \\
0 \\
0 \\
0 \\
1
\end{array}\right)\right\} \\
& S_{*}^{P_{1}}=C_{*}^{P_{1}} \quad S_{*}^{P_{2}}=C_{*}^{P_{2}} \quad S_{*}^{P_{3}}=C_{*}^{P_{3}} \\
& \left(S_{*}^{P_{1}}\right)^{\perp}=\left(C_{*}^{P_{1}}\right)^{\perp} \quad\left(S_{*}^{P_{2}}\right)^{\perp}=\left(C_{*}^{P_{2}}\right)^{\perp} \quad\left(S_{*}^{P_{3}}\right)^{\perp}=\left(C_{*}^{P_{3}}\right)^{\perp} \\
& S_{m *}^{P_{1}}=C_{*}^{P_{1}} \quad S_{m *}^{P_{2}}=C_{*}^{P_{2}} \quad S_{m *}^{P_{3}}=C_{*}^{P_{3}} \\
& \left(S_{m *}^{P_{1}}\right)^{\perp}=\left(C_{*}^{P_{1}}\right)^{\perp} \quad\left(S_{m *}^{P_{2}}\right)^{\perp}=\left(C_{*}^{P_{2}}\right)^{\perp} \quad\left(S_{m *}^{P_{3}}\right)^{\perp}=\left(C_{*}^{P_{3}}\right)^{\perp}
\end{aligned}
$$

Le découplage de l'effet des défauts n'est pas à réaliser car, dans le cadre de défauts capteur, aucun couplage n'existe. En effet, les défauts capteur ne se propagent pas et ceci est mis en relief par $\operatorname{dim}\left(C_{*}^{P_{j}}\right)=1$. Quant au diagnostic et plus particulièrement à l'isolation des 
défauts, l'étude est particulièrement simple puisque :

$$
\begin{aligned}
& \left(\frac{\partial y_{x 1}(t)}{\partial x(t)}\right)^{T}=\left(\begin{array}{llllll}
1 & 0 & 0 & 1 & 0 & 0
\end{array}\right)^{T} \subseteq\left(S_{*}^{P_{2}}\right)^{\perp} \cap\left(S_{*}^{P_{3}}\right)^{\perp} \nsubseteq\left(S_{*}^{P_{1}}\right)^{\perp} \\
& \left(\frac{\partial y_{x 2}(t)}{\partial x(t)}\right)^{T}=\left(\begin{array}{llllll}
0 & 1 & 0 & 0 & 1 & 0
\end{array}\right)^{T} \subseteq\left(S_{*}^{P_{1}}\right)^{\perp} \cap\left(S_{*}^{P_{3}}\right)^{\perp} \nsubseteq\left(S_{*}^{P_{2}}\right)^{\perp} \\
& \left(\frac{\partial y_{x 3}(t)}{\partial x(t)}\right)^{T}=\left(\begin{array}{llllll}
0 & 0 & 1 & 0 & 0 & 1
\end{array}\right)^{T} \subseteq\left(S_{*}^{P_{1}}\right)^{\perp} \cap\left(S_{*}^{P_{2}}\right)^{\perp} \nsubseteq\left(S_{*}^{P_{3}}\right)^{\perp}
\end{aligned}
$$

Suite à cette analyse, le filtre suivant est synthétisé :

$$
\Sigma_{F N L_{w_{c i}}}:\left\{\begin{array}{l}
\dot{z}_{1}(t)=-2 C_{1} \sqrt{z_{1}(t)-z_{3}(t)}+u_{1}(t) / S \\
\dot{z}_{2}(t)=2 C_{3} \sqrt{z_{3}(t)-z_{2}(t)}-2 C_{2} \sqrt{z_{2}(t)}+u_{2}(t) / S \\
\dot{z}_{3}(t)=2 C_{1} \sqrt{z_{1}(t)-z_{3}(t)}-2 C_{3} \sqrt{z_{3}(t)-z_{2}(t)} \\
\dot{z}_{4}(t)=-\Gamma_{1} z_{4}(t) \\
\dot{z}_{5}(t)=-\Gamma_{2} z_{5}(t) \\
\dot{z}_{6}(t)=-\Gamma_{3} z_{6}(t) \\
y_{z 1}(t)=z_{1}(t)+z_{4}(t) \\
y_{z 2}(t)=z_{2}(t)+z_{5}(t) \\
y_{z 3}(t)=z_{3}(t)+z_{6}(t)
\end{array}\right.
$$

où $i=\{1,2,3\}$ et les états estimés $z_{4}(t), z_{5}(t)$ et $z_{6}(t)$ sont constamment nuls.

Aucune injection de sortie n'est nécessaire pour découpler l'état de l'effet des défauts. Ainsi, ce filtre peut être considéré comme un filtre isolant chacun des défauts mais aussi comme un filtre d'isolation de l'ensemble des défauts.

En revanche, cette distinction se justifie lors de l'analyse de la convergence qui est développée dans la section suivante.

\subsection{Analyse de la convergence}

A l'aide de la théorie de la contraction (section 3.3), nous avons montré que l'étude de la convergence revient à étudier la matrice jacobienne du système. Par ailleurs, nous avons montré (section 3.3.3) l'intérêt d'augmenter l'injection de sortie. Si la convergence est prouvée, les états estimés convergent exponentiellement vers les états réels du système (en l'absence de défauts), malgré des erreurs d'initialisation.

Pour les filtres précédents (équations (4.11), (4.12), (4.14) et (4.18)), nous définissons la forme de chacune des injections de sortie augmentées. 


\subsubsection{Etude de la convergence du filtre isolant un défaut actionneur}

Nous appliquons la méthode menant aux résultats décrits dans la section 3.3.3 et plus particulièrement à la définition de l'injection de sortie augmentée. Cette dernière doit satisfaire des contraintes ( $c f$. équation (3.97)) dues d'une part aux notions de trajectoires et d'autre part aux découplages.

\section{Méthode se fondant sur $S_{*}^{P_{j}}$}

Le filtre réalisant le découplage de l'effet du défaut $w_{a 1}(t)$ muni de l'injection de sortie augmentée s'écrit :

$$
\Sigma_{F N L w_{a 1}}:\left\{\begin{array}{l}
\dot{z}_{1}(t)=-2 C_{1} \sqrt{z_{1}(t)-z_{3}(t)}+u_{1}(t) / S+\Psi_{1}^{a}\left(z(t), y_{x}(t), y_{z}(t)\right) \\
\dot{z}_{2}(t)=2 C_{3} \sqrt{z_{3}(t)-z_{2}(t)}-2 C_{2} \sqrt{z_{2}(t)}+u_{2}(t) / S+\Psi_{2}^{a}\left(z(t), y_{x}(t), y_{z}(t)\right) \\
\dot{z}_{3}(t)=2 C_{1} \sqrt{y_{x 1}(t)-z_{3}(t)}-2 C_{3} \sqrt{z_{3}(t)-z_{2}(t)}+\Psi_{3}^{a}\left(z(t), y_{x}(t), y_{z}(t)\right) \\
y_{z 1}(t)=z_{1}(t) \\
y_{z 2}(t)=z_{2}(t) \\
y_{z 3}(t)=z_{3}(t)
\end{array}\right.
$$

Si nous respectons les contraintes structurelles associée à l'injection de sortie augmentée, les égalités suivantes doivent être vérifiées :

$$
\begin{array}{r}
\Psi_{\bullet}^{a}\left(z(t), y_{x}(t), y_{z}(t)\right)=\Psi_{\bullet}^{a}\left(z(t), y_{x}(t)\right)-\Psi_{\bullet}^{a}\left(z(t), y_{z}(t)\right), \text { avec } \bullet=\{1,2,3\} \\
\Psi_{\bullet}^{a}\left(z(t), y_{x}(t), y_{z}(t)\right)=\Psi_{\bullet}^{a}\left(\overline{z_{1}}(t), \overline{y_{x 1}}(t), \overline{y_{z 1}}(t)\right), \text { avec } \bullet=\{2,3\}
\end{array}
$$

où pour tout signal $v_{\bullet}(t), \bar{v}_{\bullet}(t)=\left[v_{1}(t), \cdots, v_{\bullet-1}(t), v_{\bullet+1}(t), \cdots, v_{n}(t)\right]$. Ainsi la matrice jacobienne du filtre s'écrit comme suit :

$$
J a c=\left(\begin{array}{ccc}
-\alpha_{1}+\beta_{1} & 0+\beta_{2} & \alpha_{1}+\beta_{3} \\
0 & -\left(\alpha_{2}+\alpha_{3}\right)+\beta_{4} & \alpha_{3}+\beta_{5} \\
0 & \alpha_{3}+\beta_{6} & -\left(\alpha_{1}+\alpha_{3}\right)+\beta_{7}
\end{array}\right)
$$

avec la matrice $\beta=\frac{\partial}{\partial x(t)}\left(\Psi_{1}^{a} \Psi_{2}^{a} \Psi_{3}^{a}\right)^{T}$ constituée des différents éléments $\beta_{j}$.

Dans un premier temps, nous montrons que choisir $\beta_{6}=-\alpha_{3}$ permet d'écrire la matrice jacobienne sous forme de blocs qui n'est pas sans rappeler l'écriture d'une combinaison hiérarchique :

$$
J a c=\left(\begin{array}{c|c}
\hline B_{1} & B_{2} \\
\hline 0 & B_{3}
\end{array}\right)=\left(\begin{array}{cc|c}
\hline \alpha_{1} & 0 & \alpha_{1} \\
0 & -\left(\alpha_{2}+\alpha_{3}\right) & \alpha_{3} \\
\hline 0 & 0 & -\left(\alpha_{1}+\alpha_{3}\right) \\
\hline
\end{array}\right.
$$

avec $\beta_{i}=0, i=\{1, \cdots, 5,7\}$. Dans le domaine de fonctionnement choisi, $\alpha_{i}>0, \forall i$ puisque $\alpha_{i}=C t e / \sqrt{l_{i}(t)}, \forall i$ où $C t e>0$ et $l_{i}(t)$ la mesure d'un niveau ou la différence entre 
deux niveaux. Avec $0<l_{i}(t)<\infty, \forall i$, il semble évident que $0<\alpha_{i}<\infty$. Ainsi, les éléments qui composent le bloc matriciel $B_{2}$ sont majorés. De plus, les deux blocs $B_{1}$ et $B_{3}$ sont uniformément définis négatifs car il s'agit de blocs diagonaux dont les éléments sont strictements négatifs.

Suite à cette discussion, la solution que nous proposons est la suivante :

$$
\begin{aligned}
\Psi_{1}^{a}\left(z(t), y_{x}(t), y_{z}(t)\right) & =0 \\
\Psi_{2}^{a}\left(\overline{z_{1}}(t), \overline{y_{x 1}}(t), \overline{y_{z 1}}(t)\right) & =0 \\
\Psi_{3}^{a}\left(\overline{z_{1}}(t), \overline{y_{x 1}}(t), \overline{y_{z 1}}(t)\right) & =\Psi_{3}^{a}\left(z_{3}(t), y_{x 2}(t)\right)-\Psi_{3}^{a}\left(z_{3}(t), y_{z 2}(t)\right) \\
& =2 C_{3} \sqrt{z_{3}(t)-y_{z 2}(t)}-2 C_{3} \sqrt{z_{3}(t)-y_{x 2}(t)}
\end{aligned}
$$

Ceci permet de récrire le filtre :

$$
\Sigma_{F N L w_{a 1}}:\left\{\begin{array}{l}
\dot{z}_{1}(t)=-2 C_{1} \sqrt{z_{1}(t)-z_{3}(t)}+u_{1}(t) / S \\
\dot{z}_{2}(t)=2 C_{3} \sqrt{z_{3}(t)-z_{2}(t)}-2 C_{2} \sqrt{z_{2}(t)}+u_{2}(t) / S \\
\dot{z}_{3}(t)=2 C_{1} \sqrt{y_{x 1}(t)-z_{3}(t)}-2 C_{3} \sqrt{z_{3}(t)-y_{x 2}(t)} \\
y_{z 1}(t)=z_{1}(t) \\
y_{z 2}(t)=z_{2}(t) \\
y_{z 3}(t)=z_{3}(t)
\end{array}\right.
$$

Ce filtre assure la convergence exponentielle des estimations de l'état vers l'état réel du système malgré une mauvaise initialisation. En d'autres termes, $z(t) \longrightarrow x(t)$ avec $w_{a 1}(t)=$ $w_{a 2}(t)=0$ pour tout $z(t=0)$ et $\left(z_{2}(t), z_{3}(t)\right) \longrightarrow\left(x_{2}(t), x_{3}(t)\right) \forall w_{a 1}(t)$, avec $w_{a 2}(t)=0$ pour tout $z(t=0)$ sont les deux points forts de l'étude de la convergence.

Pour la synthèse du filtre menant à l'isolation du défaut $w_{a 1}(t)$, une étude similaire à la précédente est menée et le filtre muni d'une injection de sortie augmentée s'écrit alors comme suit :

$$
\Sigma_{F N L w_{a 2}}:\left\{\begin{array}{l}
\dot{z}_{1}(t)=-2 C_{1} \sqrt{z_{1}(t)-z_{3}(t)}+u_{1}(t) / S \\
\dot{z}_{2}(t)=2 C_{3} \sqrt{z_{3}(t)-z_{2}(t)}-2 C_{2} \sqrt{z_{2}(t)}+u_{2}(t) / S \\
\dot{z}_{3}(t)=2 C_{1} \sqrt{y_{x 1}(t)-z_{3}(t)}-2 C_{3} \sqrt{z_{3}(t)-y_{x 2}(t)} \\
y_{z 1}(t)=z_{1}(t) \\
y_{z 2}(t)=z_{2}(t) \\
y_{z 3}(t)=z_{3}(t)
\end{array}\right.
$$

Ainsi, ce filtre permet d'assurer la convergence exponentielle des estimations malgré une mauvaise initialisation, i.e. $z(t) \longrightarrow x(t)$ avec $w_{a 1}(t)=w_{a 2}(t)=0$ pour tout $z(t=0)$ et $\left(z_{2}(t), z_{3}(t)\right) \longrightarrow\left(x_{2}(t), x_{3}(t)\right) \forall w_{a 1}(t)$, avec $w_{a 2}(t)=0$ pour tout $z(t=0)$.

En remarque, nous ajoutons que ce filtre est exactement identique au précédent en raison de la symétrie du système physique étudié. En effet, la cuve 3 joue le rôle de cuve tampon tant pour la cuve 1 que pour la cuve 2, ce rôle médian constituant la symétrie. 
Méthode se fondant sur $S_{m *}^{P_{j}}$

Dans le paragraphe 4.3.1.1, nous avons synthétisé un filtre découplant totalement le défaut $w_{a 1}(t)$ (4.12). Sur cette base, nous cherchons la forme d'une injection de sortie augmentée qui assure la convergence des estimations des états du filtre vers les états réels du système :

$$
\Sigma_{F N L w_{a 1}}: \begin{cases}\dot{z}_{1}(t) & =-2 C_{1} \sqrt{z_{1}(t)-z_{3}(t)}+u_{1}(t) / S \\ & +S\left(\dot{y}_{x 1}(t)-u_{1}(t) / S+2 C_{1} \sqrt{y_{x 1}(t)-y_{x 3}(t)}\right)+\Psi_{a}^{1}\left(z(t), y_{x}(t), y_{z}(t)\right) \\ \dot{z}_{2}(t) & =2 C_{3} \sqrt{z_{3}(t)-z_{2}(t)}-2 C_{2} \sqrt{z_{2}(t)}+u_{2}(t) / S+\Psi_{a}^{2}\left(z(t), y_{x}(t), y_{z}(t)\right) \\ \dot{z}_{3}(t) & =2 C_{1} \sqrt{z_{1}(t)-z_{3}(t)}-2 C_{3} \sqrt{z_{3}(t)-z_{2}(t)}+\Psi_{a}^{3}\left(z(t), y_{x}(t), y_{z}(t)\right) \\ y_{z 1}(t) & =z_{1}(t) \\ y_{z 2}(t) & =z_{2}(t) \\ y_{z 3}(t) & =z_{3}(t)\end{cases}
$$

La matrice jacobienne associée à ce filtre s'écrit comme suit :

$$
\left(\begin{array}{ccc}
-\alpha_{1}+\beta_{1} & 0+\beta_{2} & \alpha_{1}+\beta_{3} \\
0+\beta_{4} & -\left(\alpha_{2}+\alpha_{3}\right)+\beta_{5} & \alpha_{3}+\beta_{6} \\
\alpha_{1}+\beta_{7} & \alpha_{3}+\beta_{8} & -\left(\alpha_{1}+\alpha_{3}\right)+\beta_{9}
\end{array}\right)
$$

Pour le filtre précédent, nous proposons la solution suivante (ségmentation de la matrice jacobienne (4.22)) :

$$
\begin{aligned}
\Psi_{1}^{a}\left(z(t), y_{x}(t), y_{z}(t)\right) & =0 \\
\Psi_{2}^{a}\left(z(t), y_{x}(t), y_{z}(t)\right) & =0 \\
\Psi_{3}^{a}\left(z(t), y_{x}(t), y_{z}(t)\right) & =\Psi_{3}^{a}\left(z_{3}(t), y_{x 1}(t)-\Psi_{3}^{a}\left(z_{3}(t), y_{z 1}(t)\right)+\Psi_{3}^{a}\left(z_{3}(t), y_{x 2}(t)\right)-\Psi_{3}^{a}\left(z_{3}(t), y_{z 2}(t)\right)\right. \\
& =2 C_{1} \sqrt{y_{x 1}(t)-z_{3}(t)}-2 C_{3} \sqrt{z_{3}(t)-y_{x 2}(t)} \\
& -\left(2 C_{1} \sqrt{y_{z 1}(t)-z_{3}(t)}-2 C_{3} \sqrt{z_{3}(t)-y_{z 2}(t)}\right)
\end{aligned}
$$

Nous récrivons le filtre comme suit :

$$
\Sigma_{F N L w_{a 1}}:\left\{\begin{array}{l}
\dot{z}_{1}(t)=-2 C_{1} \sqrt{z_{1}(t)-z_{3}(t)}+u_{1} / S+S\left(\dot{y}_{x 1}(t)-u_{1}(t) / S+2 C_{1} \sqrt{y_{x 1}(t)-y_{x 3}(t)}\right) \\
\dot{z}_{2}(t)=2 C_{3} \sqrt{z_{3}(t)-z_{2}(t)}-2 C_{2} \sqrt{z_{2}(t)}+u_{2}(t) / S \\
\dot{z}_{3}(t)=2 C_{1} \sqrt{y_{x 1}(t)-z_{3}(t)}-2 C_{3} \sqrt{z_{3}(t)-y_{x 2}(t)} \\
y_{z 1}(t)=z_{1}(t) \\
y_{z 2}(t)=z_{2}(t) \\
y_{z 3}(t)=z_{3}(t)
\end{array}\right.
$$

Ce filtre assure la convergence exponentielle des estimations de l'état vers l'état réel du système malgré une mauvaise initialisation du filtre, c'est-à-dire $z(t) \longrightarrow x(t) \forall w_{a 1}(t)$, avec 
$w_{a 2}(t)=0$ pour tout $z(t=0)$.

Une étude analogue permet, à l'aide d'une injection de sortie judicieusement choisie, de prouver la convergence du filtre (4.11).

\subsubsection{Etude de la convergence du filtre isolant tous les défauts ac- tionneur}

Nous rappelons l'écriture du filtre assurant l'isolation des défauts actionneur (4.15) dans sa base d'origine :

$$
\Sigma_{F N L w_{a}}:\left\{\begin{array}{l}
\dot{z}_{1}(t)=-2 C_{1} \sqrt{z_{1}(t)-z_{3}(t)}+u_{1}(t) / S \\
\dot{z}_{2}(t)=2 C_{3} \sqrt{z_{3}(t)-z_{2}(t)}-2 C_{2} \sqrt{z_{2}(t)}+u_{2}(t) / S \\
\dot{z}_{3}(t)=2 C_{1} \sqrt{y_{x 1}(t)-z_{3}(t)}-2 C_{3} \sqrt{z_{3}(t)-y_{x 2}(t)} \\
y_{z 1}(t)=z_{1}(t) \\
y_{z 2}(t)=z_{2}(t) \\
y_{z 3}(t)=z_{3}(t)
\end{array}\right.
$$

Or ce filtre possède la même matrice jacobienne que celle du filtre précédent (4.29). Il n'est donc pas nécessaire d'augmenter l'injection de sortie pour prouver la convergence du filtre.

Ce filtre assure la convergence exponentielle des estimations de l'état vers l'état réel du système malgré une mauvaise initialisation. En d'autres termes, le filtre possède les propriétés suivantes : $z_{1}(t) \longrightarrow x_{1}(t) \forall w_{a 2}(t)$ avec $w_{a 1}(t)=0$, pour tout $z(t=0)$, de même $z_{2}(t) \longrightarrow x_{2}(t) \forall w_{a 1}(t)$, avec $w_{a 2}(t)=0$ pour tout $z(t=0)$.

Nous nous sommes intéressés à l'analyse de la convergence des filtres dédiés à l'isolation des défauts actionneur. Dans les sections suivantes, nous cherchons une expression explicite des injections de sortie augmentées assurant la convergence des estimations des filtres dédiés aux défauts capteur.

\subsubsection{Etude de la convergence du filtre isolant un défaut capteur}

Nous supposons que la dynamique du filtre est identique à celle du système car les défauts capteur sont naturellement découplés. Ainsi, ce filtre, muni d'une injection de sortie 
augmentée, s'écrit comme suit :

$$
\Sigma_{F N L_{w_{c 1}}}:\left\{\begin{array}{l}
\dot{z}_{1}(t)=-2 C_{1} \sqrt{z_{1}(t)-z_{3}(t)}+u_{1}(t) / S+\Psi_{1}^{a}\left(\overline{z_{4}}(t), \overline{y_{x 1}}(t), \overline{y_{z 1}}(t)\right) \\
\dot{z}_{2}(t)=2 C_{3} \sqrt{z_{3}(t)-z_{2}(t)}-2 C_{2} \sqrt{z_{2}(t)}+u_{2}(t) / S+\Psi_{2}^{a}\left(\overline{z_{4}}(t), \overline{y_{x 1}}(t), \overline{y_{z 1}}(t)\right) \\
\dot{z}_{3}(t)=2 C_{1} \sqrt{z_{1}(t)-z_{3}(t)}-2 C_{3} \sqrt{z_{3}(t)-z_{2}(t)}+\Psi_{3}^{a}\left(\overline{z_{4}}(t), \overline{y_{x 1}}(t), \overline{y_{z 1}}(t)\right) \\
\dot{z}_{4}(t)=-\Gamma_{1} z_{4}(t)+\Psi_{4}^{a}\left(z(t), y_{x}(t), y_{z}(t)\right) \\
\dot{z}_{5}(t)=-\Gamma_{2} z_{5}(t)+\Psi_{5}^{a}\left(\overline{z_{4}}(t), \overline{y_{x 1}}(t), \overline{y_{z 1}}(t)\right) \\
\dot{z}_{6}(t)=-\Gamma_{3} z_{6}(t)+\Psi_{6}^{a}\left(\overline{z_{4}}(t), \overline{y_{x 1}}(t), \overline{y_{z 1}}(t)\right) \\
y_{z 1}(t)=z_{1}(t)+z_{4}(t) \\
y_{z 2}(t)=z_{2}(t)+z_{5}(t) \\
y_{z 3}(t)=z_{3}(t)+z_{6}(t)
\end{array}\right.
$$

Remarquons les restrictions en terme de signaux utilisables $\left(\overline{z_{4}}(t), \overline{y_{x 1}}(t), \overline{y_{z 1}}(t)\right)$ dans les écritures des injections de sorties augmentées. Celles-ci s'expliquent par le fait que le filtre est synthétisé dans le but de restreindre l'effet du défaut capteur $w_{c 1}(t)$ à la seule sortie $y_{z 1}(t)$. Ces restrictions s'observent au niveau de l'écriture de la matrice jacobienne :

$$
\left(\begin{array}{cccccc}
-\alpha_{1}+\beta_{1} & \beta_{2} & \alpha_{1}+\beta_{3} & 0 & \beta_{4} & \beta_{5} \\
\beta_{6} & -\left(\alpha_{2}+\alpha_{3}\right)+\beta_{7} & \alpha_{3}+\beta_{8} & 0 & \beta_{9} & \beta_{10} \\
\alpha_{1}+\beta_{11} & \alpha_{3}+\beta_{12} & -\left(\alpha_{1}+\alpha_{3}\right)+\beta_{13} & 0 & \beta_{14} & \beta_{15} \\
\beta_{17} & \beta_{18} & \beta_{19} & -\Gamma_{1}+\beta_{20} & \beta_{21} & \beta_{22} \\
\beta_{23} & \beta_{24} & \beta_{25} & 0 & -\Gamma_{2}+\beta_{26} & \beta_{27} \\
\beta_{28} & \beta_{29} & \beta_{30} & 0 & \beta_{31} & -\Gamma_{3}+\beta_{32}
\end{array}\right)
$$

Pour assurer la convergence, nous proposons la solution suivante :

$$
\begin{aligned}
& \Psi_{1}^{a}\left(\overline{z_{4}}(t), \overline{y_{x 1}}(t), \overline{y_{z 1}}(t)\right)=-2 C_{1} \sqrt{z_{1}(t)-y_{x 3}(t)}+2 C_{1} \sqrt{z_{1}(t)-y_{z 3}(t)} \\
& \Psi_{2}^{a}\left(\overline{z_{4}}(t), \overline{y_{x 1}}(t), \overline{y_{z 1}}(t)\right)=2 C_{3} \sqrt{y_{x 3}(t)-z_{2}(t)}-2 C_{3} \sqrt{y_{z 3}(t)-z_{2}(t)} \\
& \Psi_{3}^{a}\left(\overline{z_{4}}(t), \overline{y_{x 1}}(t), \overline{y_{z 1}}(t)\right)=0 \\
& \Psi_{4}^{a}\left(z(t), y_{x}(t), y_{z}(t)\right)=0 \\
& \Psi_{5}^{a}\left(\overline{z_{4}}(t), \overline{y_{x 1}}(t), \overline{y_{z 1}}(t)\right)=0 \\
& \Psi_{6}^{a}\left(\overline{z_{4}}(t), \overline{y_{x 1}}(t), \overline{y_{z 1}}(t)\right)=0
\end{aligned}
$$


Le filtre isolant le défaut capteur 1 s'écrit alors :

$$
\Sigma_{F N L_{w_{c 1}}}:\left\{\begin{array}{l}
\dot{z}_{1}(t)=-2 C_{1} \sqrt{z_{1}(t)-y_{x 3}(t)}+u_{1}(t) / S \\
\dot{z}_{2}(t)=2 C_{3} \sqrt{y_{x 3}(t)-z_{2}(t)}-2 C_{2} \sqrt{z_{2}(t)}+u_{2}(t) / S \\
\dot{z}_{3}(t)=2 C_{1} \sqrt{z_{1}(t)-z_{3}(t)}-2 C_{3} \sqrt{z_{3}(t)-z_{2}(t)} \\
\dot{z}_{4}(t)=-\Gamma_{1} z_{4}(t) \\
\dot{z}_{5}(t)=-\Gamma_{2} z_{5}(t) \\
\dot{z}_{6}(t)=-\Gamma_{3} z_{6}(t) \\
y_{z 1}(t)=z_{1}(t)+z_{4}(t) \\
y_{z 2}(t)=z_{2}(t)+z_{5}(t) \\
y_{z 3}(t)=z_{3}(t)+z_{6}(t)
\end{array}\right.
$$

et assure la convergence exponentielle des estimations de l'état vers l'état réel du système malgré une mauvaise initialisation du filtre. En d'autres termes, le filtre possède les propriétés suivantes : $z_{4}(t) \longrightarrow x_{4}(t) \forall w_{c 2}(t), w_{c 3}(t)$ avec $w_{c 1}(t)=0$, pour tout $z(t=0)$, de même $\left(\begin{array}{llllllll}z_{1}(t) & z_{2}(t) & z_{3}(t) & z_{5}(t) & z_{6}(t)\end{array}\right) \longrightarrow\left(\begin{array}{lllll}x_{1}(t) & x_{2}(t) & x_{3}(t) & x_{5}(t) & x_{6}(t)\end{array}\right) \quad \forall w_{c 1}(t)$, avec $w_{c 2}(t)=w_{c 3}(t)=0$ pour tout $z(t=0)$.

A l'aide d'études similaires, nous proposons deux filtres isolant les deux autres défauts capteur.

Le filtre assurant l'isolation du défaut capteur 2 s'écrit comme suit :

$$
\Sigma_{F N L_{w_{c 2}}}:\left\{\begin{array}{l}
\dot{z}_{1}(t)=-2 C_{1} \sqrt{z_{1}(t)-y_{x 3}(t)}+u_{1}(t) / S \\
\dot{z}_{2}(t)=2 C_{3} \sqrt{y_{x 3}(t)-z_{2}(t)}-2 C_{2} \sqrt{z_{2}(t)}+u_{2}(t) / S \\
\dot{z}_{3}(t)=2 C_{1} \sqrt{z_{1}(t)-z_{3}(t)}-2 C_{3} \sqrt{z_{3}(t)-z_{2}(t)} \\
\dot{z}_{4}(t)=-\Gamma_{1} z_{4}(t) \\
\dot{z}_{5}(t)=-\Gamma_{2} z_{5}(t) \\
\dot{z}_{6}(t)=-\Gamma_{3} z_{6}(t) \\
y_{z 1}(t)=z_{1}(t)+z_{4}(t) \\
y_{z 2}(t)=z_{2}(t)+z_{5}(t) \\
y_{z 3}(t)=z_{3}(t)+z_{6}(t)
\end{array}\right.
$$


et le filtre suivant isole le défaut capteur 3 :

$$
\Sigma_{F N L_{w_{c 3}}}:\left\{\begin{array}{l}
\dot{z}_{1}(t)=-2 C_{1} \sqrt{z_{1}(t)-z_{3}(t)}+u_{1}(t) / S \\
\dot{z}_{2}(t)=2 C_{3} \sqrt{z_{3}(t)-z_{2}(t)}-2 C_{2} \sqrt{z_{2}(t)}+u_{2}(t) / S \\
\dot{z}_{3}(t)=2 C_{1} \sqrt{y_{x 1}(t)-z_{3}(t)}-2 C_{3} \sqrt{z_{3}(t)-y_{x 2}(t)} \\
\dot{z}_{4}(t)=-\Gamma_{1} z_{4}(t) \\
\dot{z}_{5}(t)=-\Gamma_{2} z_{5}(t) \\
\dot{z}_{6}(t)=-\Gamma_{3} z_{6}(t) \\
y_{z 1}(t)=z_{1}(t)+z_{4}(t) \\
y_{z 2}(t)=z_{2}(t)+z_{5}(t) \\
y_{z 3}(t)=z_{3}(t)+z_{6}(t)
\end{array}\right.
$$

En remarque, nous ajoutons que les 3 filtres synthétisés dans cette section s'inscrivent tout à fait dans une démarche visant la synthèse d'un banc d'observateurs G.O.S. (définie au paragraphe 1.3.2.3).

Lors de l'analyse structurelle, nous insistons sur la forme du filtre unique (4.18) qui permet l'isolation de l'ensemble des défauts. Dans la section suivante, nous étudions la convergence d'un tel filtre.

\subsubsection{Etude de la convergence du filtre isolant tous les défauts cap- teur}

Le filtre (4.18) muni d'une injection de sortie augmentée s'écrit :

$$
\Sigma_{F N L_{w_{c}}}:\left\{\begin{array}{l}
\dot{z}_{1}(t)=-2 C_{1} \sqrt{z_{1}(t)-z_{3}(t)}+u_{1}(t) / S \\
\dot{z}_{2}(t)=2 C_{3} \sqrt{z_{3}(t)-z_{2}(t)}-2 C_{2} \sqrt{z_{2}(t)}+u_{2}(t) / S \\
\dot{z}_{3}(t)=2 C_{1} \sqrt{z_{1}(t)-z_{3}(t)}-2 C_{3} \sqrt{z_{3}(t)-z_{2}(t)} \\
\dot{z}_{4}(t)=-\Gamma_{1}\left(z_{4}(t)\right)+\Psi_{1}^{a}\left(z_{4}(t), y_{x 1}(t), y_{z 1}(t)\right) \\
\dot{z}_{5}(t)=-\Gamma_{2}\left(z_{5}(t)\right)+\Psi_{2}^{a}\left(z_{5}(t), y_{x 2}(t), y_{z 2}(t)\right) \\
\dot{z}_{6}(t)=-\Gamma_{3}\left(z_{6}(t)\right)+\Psi_{3}^{a}\left(z_{6}(t), y_{x 3}(t), y_{z 3}(t)\right) \\
y_{z 1}(t)=z_{1}(t)+z_{4}(t) \\
y_{z 2}(t)=z_{2}(t)+z_{5}(t) \\
y_{z 3}(t)=z_{3}(t)+z_{6}(t)
\end{array}\right.
$$


La matrice jacobienne associée à ce filtre possède la forme suivante :

$$
\left(\begin{array}{cccccc}
-\alpha_{1} & 0 & +\alpha_{1} & 0 & 0 & 0 \\
0 & -\left(\alpha_{2}+\alpha_{3}\right) & \alpha_{3} & 0 & 0 & 0 \\
\alpha_{1} & \alpha_{3} & -\left(\alpha_{1}+\alpha_{3}\right) & 0 & 0 & 0 \\
0 & 0 & 0 & -\Gamma_{1}+\beta_{1} & 0 & 0 \\
0 & 0 & 0 & 0 & -\Gamma_{2}+\beta_{2} & 0 \\
0 & 0 & 0 & 0 & 0 & -\Gamma_{3}+\beta_{3}
\end{array}\right)
$$

Il en résulte que la matrice jacobienne n'est pas uniformément définie négative quelles que soient les injections de sorties choisies. La convergence ne peut donc pas être prouvée.

Une solution à ce problème consiste à diminuer le nombre de défauts à isoler avec un filtre unique de manière à gagner des degrés de libertés dans l'écriture de l'injection de sortie augmentée. Ceci est le principe même des structures de banc d'observateurs (dédiée et généralisée définies à la section 1.3.2).

Dans la section suivante, nous illustrons ces différents résultats théoriques à l'aide de simulations.

\subsection{Simulations}

Les figures 4.2(a) et 4.2(b) représentent l'ensemble des signaux connus et mesurés du système.

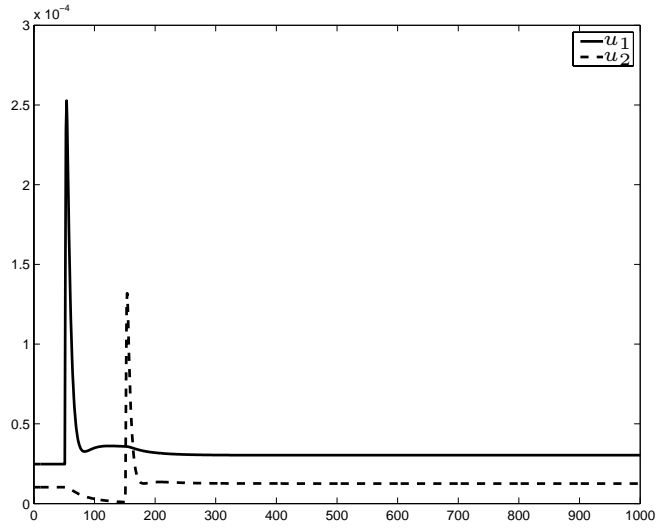

(a) Commandes

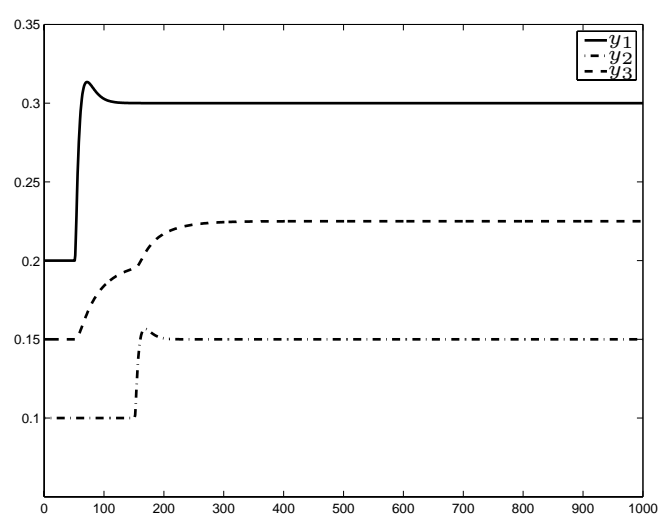

(b) Sorties du système

FIG. 4.2: Signaux connus du système dans le cas sans défaut

La figure 4.2(a) présente le signal de commande ou plus précisément les deux débits des deux pompes (actionneurs) tandis que la deuxième 4.2(b) fait apparaître les sorties du système. Le système est en boucle ouverte, i.e. la commande n'est pas fonction des sorties du système. Toutefois, grâce à la connaissance du modèle du système, il est possible de déterminer les 
commandes $u_{1}(t)$ et $u_{2}(t)$ de façon à faire coïncider les sorties $y_{1}(t)$ et $y_{2}(t)$ aux deux fonctions de références définies comme suit :

niveau cuve $1\left\{\begin{array}{l}0 \leq t<50 \quad L_{1 \text { ref }}(t)=0.2 m \\ t \geq 50 \quad L_{1 \text { ref }}(t)=0.3 m\end{array} \quad\right.$ niveau cuve $2\left\{\begin{array}{l}0 \leq t<50 \quad L_{2 \text { ref }}(t)=0.1 m \\ t \geq 150 \quad L_{2 \text { ref }}(t)=0.15 m\end{array}\right.$

D'après la figure $4.2(b)$, nous remarquons que ces trajectoires sont suivies avec un temps de réponse relativement faible.

Ensuite, afin d'évaluer l'influence d'une mauvaise connaissance des conditions initiales, nous initialisons différemment le système du filtre :

$x(t=0)-z(t=0)=\left(\begin{array}{lll}0.1 \cdot 10^{-6} & 0.2 \cdot 10^{-6} & 0.3 \cdot 10^{-6}\end{array}\right)^{T}$.

Nous ajoutons que l'ensemble des défauts actionneur (resp. capteur) considérés lors de ces simulations sont de type biais et ont une amplitude égale à $10 \%$ de la commande (resp. de la sortie).

\subsubsection{Défauts actionneur}

Nous montrons à la figure 4.3 l'effet du défaut actionneur $w_{a 1}(t)$ sur les sorties du système.

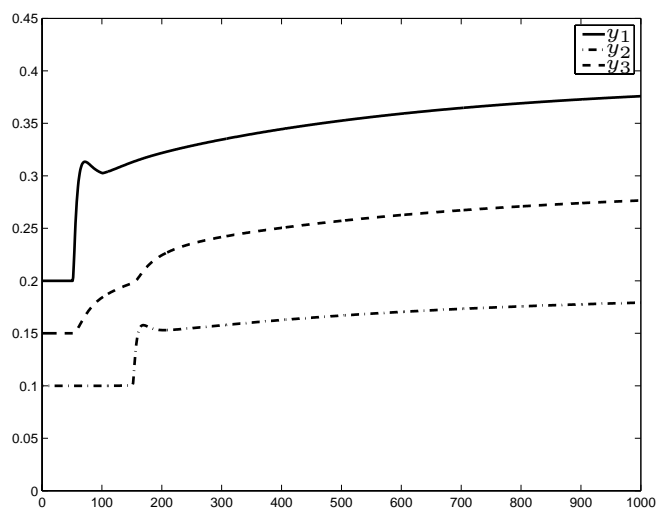

FIG. 4.3: Sortie du système dans le cas d'un défaut actionneur $w_{a 1}(t)$

Une dérive sur les trois niveaux est à noter. Puisqu'il s'agit d'un défaut agissant directement sur le débit d'entrée dans la cuve 1 , la dérive est plus franche sur ce niveau (i.e. $L_{1}$ ou $\left.z_{1}(t)\right)$. Mais puisque toutes les cuves sont connectées entre elles, le défaut influence, dans une moindre mesure, les niveaux des cuves 2 et 3 .

Les effets du défaut actionneur $w_{a 2}(t)$ étant identiques, ils ne sont pas représentés.

Les erreurs d'estimation des filtres (4.11) et (4.26) sont représentés aux figures $4.4(a)$ et 4.4(b) en présence du défaut $w_{a 1}(t)$.

La figure 4.4(a) reflète le problème lié à la convergence qui se traduit par une erreur d'estimation tendant très lentement, si tentée qu'elle le fasse, vers zéro. En revanche, l'erreur d'estimation figure $4.4(b)$ tend très rapidement vers zéro malgré l'occurrence du défaut actionneur $w_{a 1}(t)$ et illustre l'intérêt de l'étude de la convergence. Quant à la figure 4.4(c), elle 


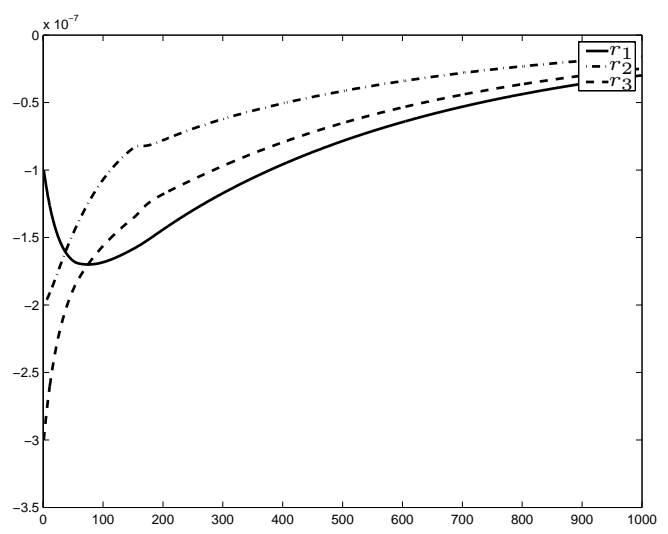

(a) En présence du défaut actionneur $w_{a 1}(t)$

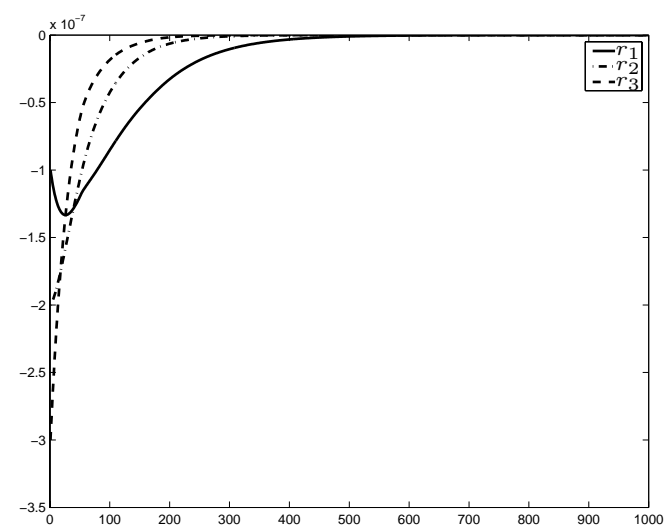

(b) En présence du défaut actionneur $w_{a 1}(t)$ et avec l'injection de sortie augmentée

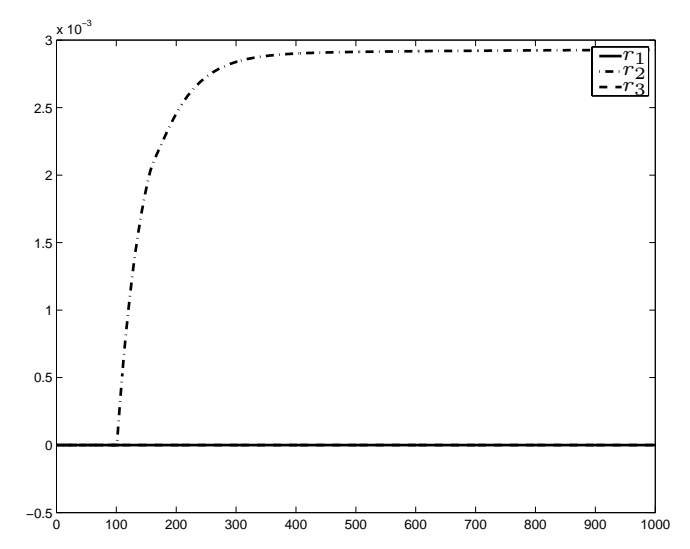

(c) En présence du défaut actionneur $w_{a 2}(t)$ et avec l'injection de sortie augmentée

FIG. 4.4: Erreur d'estimation du filtre insensible au défaut actionneur $w_{a 1}(t)$

met en exergue la sensibilité du filtre au défaut actionneur $w_{a 2}(t)$.

Puisque nous montrons qu'il est possible de synthétiser un filtre assurant l'isolation des deux défauts actionneur (4.30), nous présentons à la figure 4.5 les sorties du système 4.5( $a$ ) d'une part et l'erreur d'estimation de ce filtre d'autre part $4.5(b)$.

Notons que les défauts actionneur se traduisent par une déviation des trajectoires du système de leurs références. Mais ce qui constitue sans doute l'un des résultats majeurs est illustré par la simulation 4.5(b). En effet, à l'aide de ce seul filtre nous détectons aisément l'occurrence du défaut actionneur $w_{a 1}(t)$ à l'instant 100 et du défaut actionneur $w_{a 2}(t)$ à l'instant 200.

Lors de l'étude théorique, nous avons étudié un deuxième groupe de défauts (les défauts capteur). La section suivante présente plusieurs simulations illustrant l'isolation de ces défauts. 


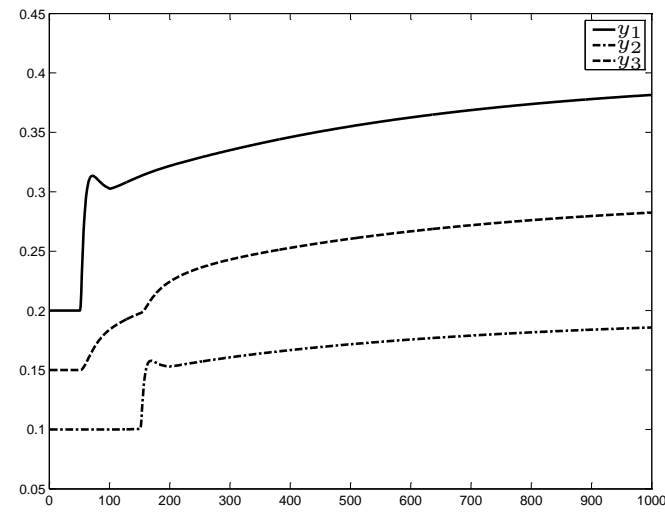

(a) Sorties du système

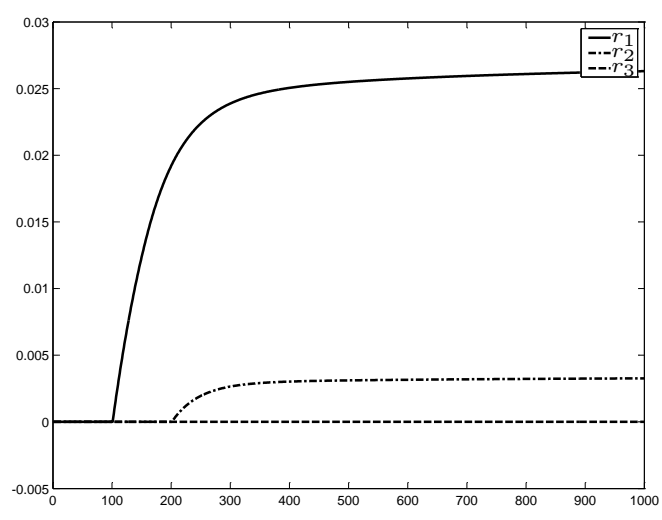

(b) Erreur d'estimation du filtre d'isolation

FIG. 4.5: Simulation de l'apparition de deux défauts consécutifs

\subsubsection{Défauts capteur}

Nous présentons les simulations du filtre qui permettent l'isolation du défaut capteur $w_{c 1}(t)$ (défini par l'équation (4.34)). La figure 4.6 met en relief, identiquement aux filtres précédents, l'analyse de convergence. Les résultats sont particulièrement probants en l'absence de défauts.

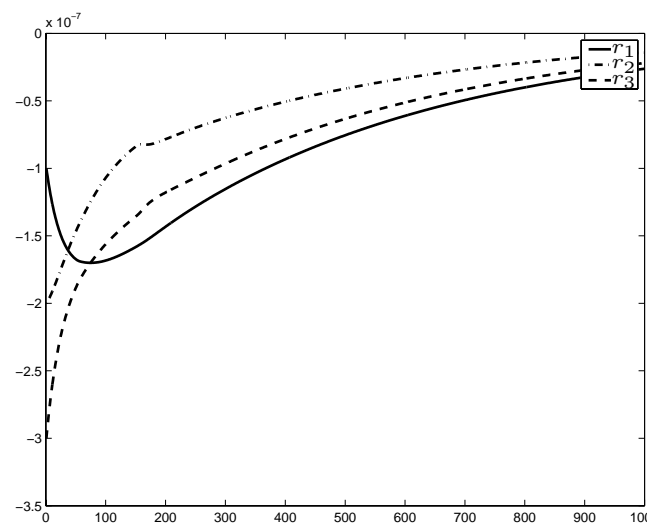

(a) Sans prouver la convergence

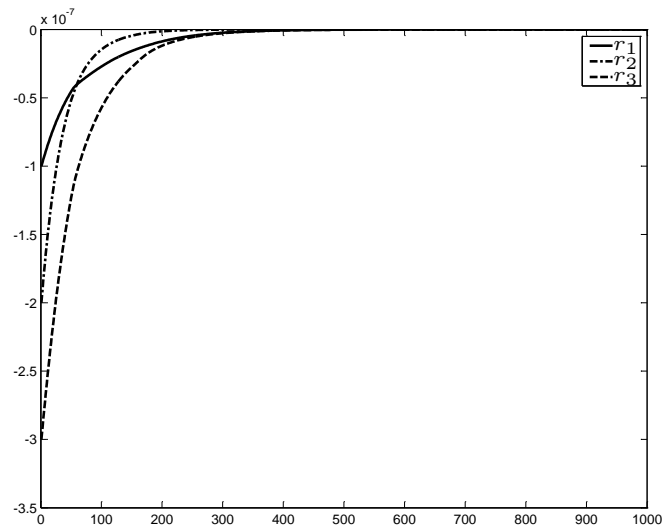

(b) En ayant prouvé la convergence

Fig. 4.6: Erreur d'estimation dans le cas sans défaut

Nous choisissons de présenter à la figure 4.7 les sorties du système en présence du défaut capteur $w_{c 1}(t)$. Il est alors trivial de reproduire l'effet des 2 autres défauts capteur.

Par la suite nous présentons plus particulièrement le comportement du filtre assurant l'isolation du défaut capteur $w_{c 1}(t)$ (figure 4.8) lors de l'occurrence de l'ensemble des défauts :

- $w_{c 1}(t)$ figure $4.8(a)$,

- $w_{c 2}(t)$ figure $4.8(b)$,

- $w_{c 3}(t)$ figure $4.8(c)$.

Une étude théorique de l'erreur d'estimation (identique au résidu pour les simulations) $r(t)=$ $y_{z}(t)-y_{x}(t)=z(t)-x(t)$ mène à différents résultats. Suite à la définition du filtre (4.31) 


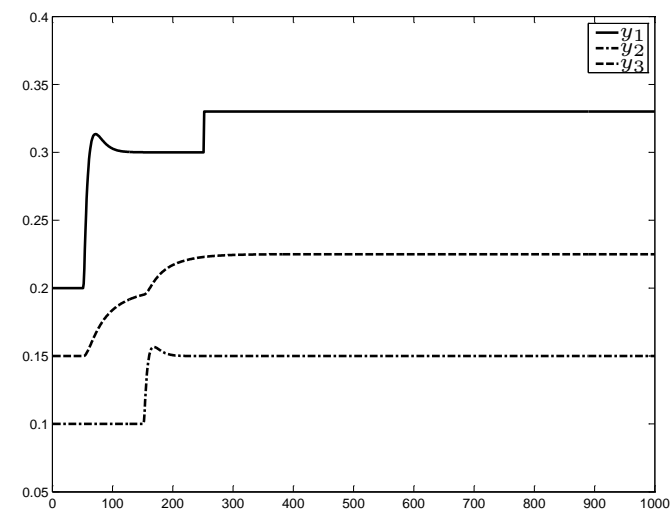

(a) Sorties du système

FIG. 4.7: Cas du défaut capteur $w_{c 1}(t)$

intégrant les contraintes dues au découplage, nous sommes en mesure de déterminer les sensibilités du résidu aux défauts. En effet, une étude de l'invariance des sous-espaces d'état permet d'établir le tableau 4.1 où les éléments qui le constituent sont à interpréter comme suit :

- "1" équivaut à une sensibilité certaine,

- "0" équivaut à une insensibilité certaine,

- " $1 / 0 "$ équivaut à une indétermination.

\begin{tabular}{|c|c|c|c|}
\hline Résidus & $w_{c 1}(t)$ & $w_{c 2}(t)$ & $w_{c 3}(t)$ \\
\hline \hline$z_{1}(t)-x_{1}(t)$ & 1 & $1 / 0$ & $1 / 0$ \\
\hline$z_{2}(t)-x_{2}(t)$ & 0 & 1 & $1 / 0$ \\
\hline$z_{3}(t)-x_{3}(t)$ & 0 & $1 / 0$ & 1 \\
\hline
\end{tabular}

TAB. 4.1: Sensibilité des résidus

Les indéterminations sont dues à la forme générale de l'injection de sortie augmentée dans l'écriture du système d'équation (4.31). En revanche, si nous précisions l'écriture du filtre à l'aide des égalités définies par les équations (4.33), alors nous sommes en mesure de lever les indéterminations et de récrire le tableau 4.1 en 4.2 . Notons la colonne de "1" du tableau qui

\begin{tabular}{|c|c|c|c|}
\hline Résidus & $w_{c 1}(t)$ & $w_{c 2}(t)$ & $w_{c 3}(t)$ \\
\hline \hline$z_{1}(t)-x_{1}(t)$ & 1 & 0 & 1 \\
\hline$z_{2}(t)-x_{2}(t)$ & 0 & 1 & 1 \\
\hline$z_{3}(t)-x_{3}(t)$ & 0 & 0 & 1 \\
\hline
\end{tabular}

TAB. 4.2: Sensibilité des résidus

s'explique par l'utilisation de la sortie $y_{x 3}(t)$ au sein de l'injection de sortie (sortie sensible au 

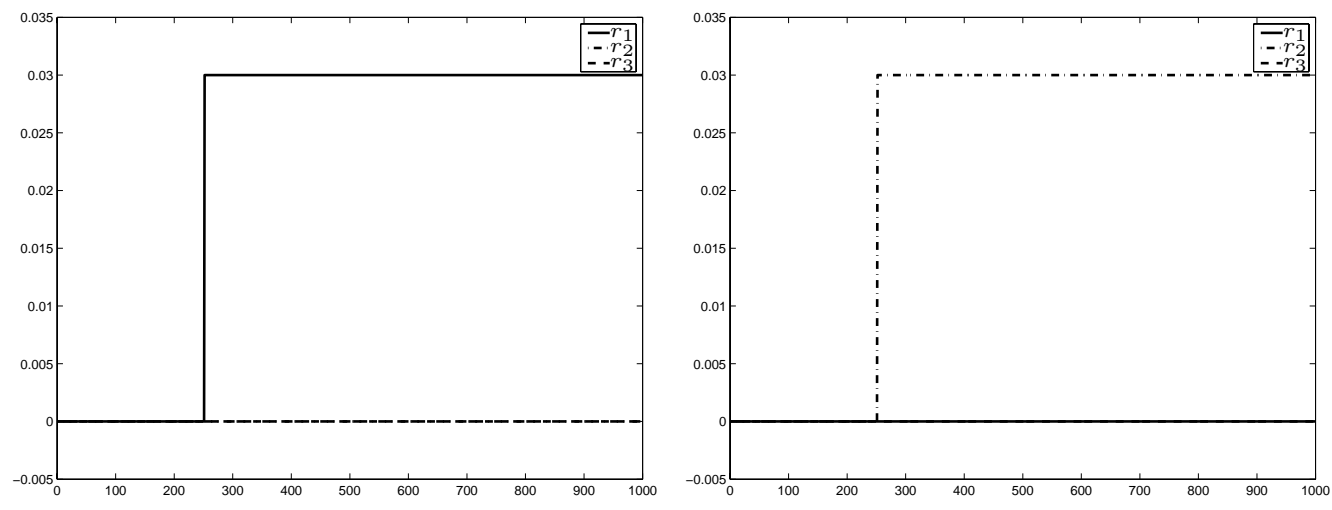

(a) Dans le cas du défaut capteur $w_{c 1}(t)$

(b) Dans le cas du défaut capteur $w_{c 2}(t)$

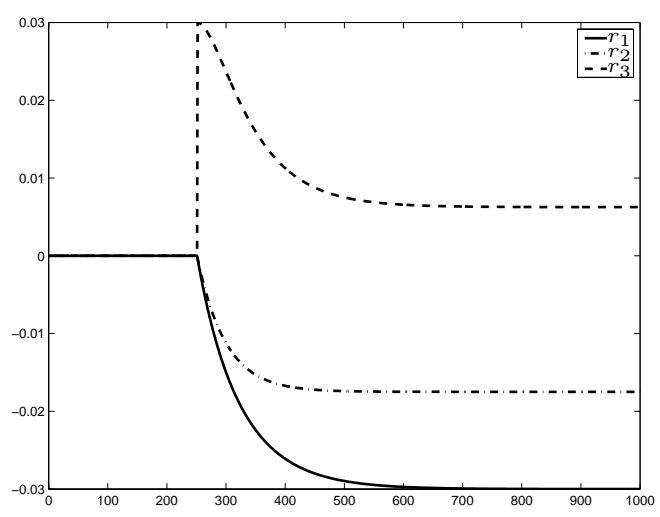

(c) Dans le cas du défaut capteur $w_{c 3}(t)$

FIG. 4.8: Erreur d'estimation du filtre 
défaut capteur $\left.w_{c 3}(t)\right)$. En revanche, puisque la sortie $y_{x 2}(t)$ n'est pas utilisée, nous assurons la seule sensibilité de $z_{2}(t)-x_{2}(t)$ à $w_{c 2}(t)$.

Le tableau 4.2 est illustré par les courbes représentées à la figure 4.8. En effet, la figure 4.8( $a)$ présente les trois résidus et seul le premier est sensible à l'occurrence du défaut $w_{c 1}(t)$. La figure 4.8(b) présente les trois résidus et seul le deuxième est sensible à l'occurrence du défaut $w_{c 2}(t)$. Quant à la figure 4.8(c) la sensibilité des trois résidus au défaut $w_{c 3}(t)$ est évidente.

\subsection{Conclusion}

Nous avons présenté dans ce chapitre l'application à un cas concret des différentes méthodes théoriques proposées dans les chapitres précédents. Nous avons choisi le système physique des 3 cuves qui possède l'avantage de présenter un couplage fort entre les différents niveaux de liquide et permet ainsi la mise en relief des résultats propres au découplage. Quatre méthodes de découplages sont présentées.

Une première méthode reflète la synthèse de filtres assurant l'isolation des défauts actionneur à l'aide des distributions introduites par C. De Persis et A. Isidori $\left(S_{*}^{P_{j}}\right)$. L'isolation des défauts n'est assurée que si 2 filtres sont synthétisés.

Une deuxième méthode consiste à découpler totalement chaque défaut actionneur. Cet objectif est réalisé grâce au calcul des distributions $S_{m *}^{P_{j}}$ (introduites au chapitre 2). Ces dernières permettent d'augmenter (en terme d'inclusion) le sous-espace insensible à un défaut. Comme précédemment, l'utilisation de 2 filtres est nécessaire à l'isolation des défauts.

En troisième méthode, nous proposons l'isolation des deux défauts actionneur à l'aide d'un seul et même filtre. Ceci nécessite la prise en compte de contraintes additionnelles (définies dans le chapitre 3) qui sont satisfaites pour cet exemple.

Enfin, une quatrième méthode visant l'isolation des défauts capteur est mise en oeuvre. Suite à l'énoncé des structures particulières de banc de filtres (chapitre 1), nous présentons et simulons un banc d'observateurs de type G.O.S.

Afin d'assurer la convergence exponentielle des estimations provenant de chaque filtre malgré la présence d'erreurs d'initialisation, nous appliquons la théorie de la contraction. Cette dernière montre, et c'est tout son intérêt, une facilité de mise en oeuvre intéressante. Les résultats obtenus en simulation sont particulièrement probants. 


\section{Chapitre 5}

\section{Ouverture : vers un formalisme algébrique du diagnostic}

\section{$5.1 \quad$ Introduction}

Ce chapitre est une ouverture tant d'un point de vue des résultats que par les notions mises en oeuvres. En effet, suite à l'école d'été de Grenoble 2001 (s'intitulant : Geometric modelling and control of physical systems) et plus précisément à l'exposé de M. Fliess, nous avons été intéressés par les outils mathématiques mis en oeuvre dans de nombreux articles ([23], [20], [22] et [19]). Ces articles traitent principalement des problèmes de commande des systèmes. Basée sur des notions algébriques avancées, la théorie des modules permet un formalisme particulièrement intéressant. Comme nous le mettons en évidence dans les chapitres 2 et 3 , de nombreuses notions sont difficiles à traduire (en terme de formalisme) à l'aide d'une approche différentielle du type espace d'état. Il serait facile d'imputer ces difficultés au fait que les systèmes traités sont non linéaires mais il n'en est rien. En effet, les mêmes difficultés sont rencontrées lors de l'étude des systèmes linéaires.

Ces difficultés sont grandement réduites grâce à l'utilisation de notions algébriques telles que la théorie des modules. En effet, les propriétés qui définissent un module ( $c f$. annexe C) semblent particulièrement appropriées à la caractérisation des systèmes.

Nous insistons sur le fait que ce chapitre d'ouverture n'est certainement pas exhaustif mais constitue en soit une perspective intéressante tant pour les systèmes linéaires que les extensions envisageables pour les systèmes non linéaires. En d'autres termes, plutôt que d'évoquer lors de la conclusion générale les possibilités intéressantes de formalisme qu'offre l'algèbre, nous les mettons en relief à l'aide de ce chapitre. Evidemment, ces caractéristiques seraient bien plus explicites dans le cadre de l'étude des systèmes non linéaires, mais ceci pourrait faire l'objet d'une future thèse. C'est pourquoi, dans ce chapitre, nous ne ferons qu'en exhiber les intérêts en considérant les systèmes linéaires.

En tant que chapitre d'ouverture, nous présentons dans un premier temps la caractérisation 
d'un système linéaire à l'aide des modules. Puis l'établissement des notions "habituelles" en automatique (commandabilité, observabilité et sorties plates) qui caractérisent un système est effectué à l'aide de la théorie des modules. Nous proposons ensuite les définitions de la détectabilité et de l'isolabilité de défauts. Enfin, nous illustrons nos résultats à l'aide d'exemples académiques.

\subsection{Représentation des systèmes linéaires à l'aide de la théorie des modules}

Dans le cadre de l'automatique, nous définissons des opérateurs particuliers qui satisfont les propriétés d'un module. Nous pouvons alors décrire les systèmes linéaires à l'aide d'un formalisme algébrique. Pour faciliter la lecture, de nombreuses définitions sont rappelées en annexe C.

\subsubsection{Définition d'un module approprié aux systèmes linéaires}

Dans ce qui suit, nous posons les opérations définissant les modules (cf. [19]).

En considérant un champ $k$ muni d'une simple dérivation $d / d t$ comme opération, nous avons :

$$
\begin{array}{r}
\forall a, b \in k, \quad \frac{d}{d t}(a+b)=\dot{a}+\dot{b} \\
\frac{d}{d t}(a b)=\dot{a} b+a \dot{b}
\end{array}
$$

il s'agit en l'occurrence d'un champ commutatif.

L'anneau ( $c f$. annexe C) des opérateurs différentiels linéaires noté $k[d / d t]$ est de la forme suivante :

$$
\sum_{\text {finie }} a_{\alpha} \frac{d^{\alpha}}{d t^{\alpha}}, \quad a_{\alpha} \in k
$$

Nous ajoutons que l'anneau $k[d / d t]$ est commutatif si, et seulement si, $k$ est un champ de constantes (i.e. $\left.\frac{d}{d t} a_{\alpha}=0\right)$.

Suite à cette remarque, nous distinguons un $\mathbb{R}[d / d t]$-module (où $k$ est le champ de constantes $\mathbb{R})$ d'un $k[d / d t]$-module.

\subsubsection{Définition d'un système linéaire à l'aide de la théorie des mo- dules}

Comme nous venons de le définir, il est possible de considérer aussi bien des coefficients variables dans le temps $(k[d / d t]$-module) menant à la définition des systèmes Linéaires Va- 
riant dans le Temps (L.T.V.) que des coefficients constants pour la définition des systèmes Linéaires Invariant dans le Temps (L.T.I.).

Si chacun d'eux est perturbé par un défaut $w$, la représentation kalmanienne d'un système L.T.V. perturbé est donnée par :

$$
\Sigma_{L T V}:\left\{\begin{array}{l}
\dot{x}(t)=A(t) x(t)+B(t) u(t)+P(t) w(t) \\
y(t)=C(t) x(t)
\end{array}\right.
$$

tandis que celle d'un système L.T.I. perturbé reflète un cas particulier :

$$
\Sigma_{L T I}:\left\{\begin{array}{l}
\dot{x}(t)=A x(t)+B u(t)+P w(t) \\
y(t)=C x(t)
\end{array}\right.
$$

où $x(t) \in \mathbb{R}^{n}, u(t) \in \mathbb{R}^{m}, y(t) \in \mathbb{R}^{p}$ et $w(t) \in \mathbb{R}^{q}$ représentent respectivement l'état, la commande, la sortie et l'entrée inconnue perturbatrice nommée défaut. Quant aux matrices $A, B, C$ et $P$ nous les considérons constantes dans le cadre d'un système L.T.I. ou fonction du temps pour un système L.T.V.

Afin de simplifier les écritures, nous considérons par la suite uniquement des systèmes L.T.I., tout en insistant sur la possibilité d'étendre tous les résultats aux systèmes L.T.V.

Soit le $\mathbb{R}[d / d t]$-module libre engendré par $\bar{x}, \bar{u}, \bar{w}$ noté $N$. Tout élément $n$ de $N$ s'écrit :

$$
n=\sum_{\text {finie }} a_{\alpha} \bar{x}^{(\alpha)}+\sum_{\text {finie }} a_{\beta} \bar{u}^{(\beta)}+\sum_{\text {finie }} a_{\gamma} \bar{w}^{(\gamma)}
$$

Soit $F$ un sous-module de $N$ engendré par les composantes du vecteur :

$$
(d / d t . I-A-B \quad-P)\left(\begin{array}{c}
\bar{x} \\
\bar{u} \\
\bar{w}
\end{array}\right)
$$

Nous définissons $\Lambda$ comme étant le $\mathbb{R}[d / d t]$-module associé à la dynamique linéaire du système. Alors $\Lambda$ doit vérifier les propriétés suivantes :

(i) $0 \longrightarrow N \longrightarrow F \longrightarrow \Lambda \longrightarrow 0$ est une suite exacte,

(ii) $\Lambda=N / F$, i.e. $\Lambda$ est le sous-module quotient de $\mathrm{N}$ par $\mathrm{F}$.

En remarque, nous ajoutons que $\left(\begin{array}{lll}x & u & w\end{array}\right)$ sont les résidus de $\left(\begin{array}{lll}\bar{x} & \bar{u} & \bar{w}\end{array}\right)$, c'est-à-dire leurs images (ou projections) canoniques dans $\Lambda$.

Néanmoins, il ne faut pas considérer que la définition du $\mathbb{R}[d / d t]$-module $\Lambda$ est exclusivement 
liée à la représentation utilisée (kalmanienne par exemple) pour décrire le système. En effet, il est toujours possible de définir des états généralisés $\eta=\left(\begin{array}{lll}\eta_{1} & \cdots & \eta_{n}\end{array}\right)^{T}$ comme des éléments de $\Lambda$ dont l'image canonique dans $\Lambda / \operatorname{span}_{\mathbb{R}[d / d t]}(u)$ est une base. Les états généralisés permettent une représentation d'état généralisée :

$$
\frac{d}{d t}\left(\begin{array}{c}
\eta_{1} \\
\vdots \\
\eta_{n}
\end{array}\right)=F\left(\begin{array}{c}
\eta_{1} \\
\vdots \\
\eta_{n}
\end{array}\right)+\sum_{\alpha=0}^{\nu} G_{\alpha} \frac{d^{\alpha}}{d t^{\alpha}}\left(\begin{array}{c}
u_{1} \\
\vdots \\
u_{m}
\end{array}\right)
$$

Suite à la définition du $\mathbb{R}[d / d t]$-module $\Lambda$ décrivant le système linéaire (5.4), nous définissons à l'aide de propriétés algébriques les caractéristiques couramment rencontrées en automatique.

\subsection{Caractérisation d'un système linéaire}

Introduisons le sous-module $\Lambda^{\text {nom }}$ de $\Lambda$ tel que $\Lambda^{\text {nom }}=\Lambda / \operatorname{span}_{\mathbb{R}[d / d t]}(w)$. En d'autres termes, ce sous-module est le reflet d'une dynamique (5.4) non perturbée $(w=0, \forall t)$.

Par hypothèse de travail, nous considérons une commande en boucle ouverte. Ainsi il semble trivial d'ajouter $: \operatorname{span}_{\mathbb{R}[d / d t]}(w) \cap \operatorname{span}_{\mathbb{R}[d / d t]}(u)=\{0\}$ donc $\operatorname{span}_{\mathbb{R}[d / d t]}(u)$ est isomorphe à sa projection canonique $\operatorname{span}_{\mathbb{R}[d / d t]}\left(u^{\text {nom }}\right)$ dans $\Lambda^{\text {nom }}$.

De plus, afin d'être en accord avec l'étude de la convergence réalisée dans le chapitre 3, nous supposons que les conditions initiales liées à chacune des équations différentielles sont :

- soit nulles,

- soit modélisées comme une perturbation.

Suite à cette remarque, nous savons que $\Lambda^{\text {nom }} / \operatorname{span}_{\mathbb{R}[d / d t]}\left(u^{\text {nom }}\right)$ est de torsion. En effet, tout élément, non influencé par la commande, l'est au moins par la perturbation.

Nous représentons un système linéaire à l'aide d'un diagramme mettant en oeuvre une suc- 
cession de suites exactes comme suit :

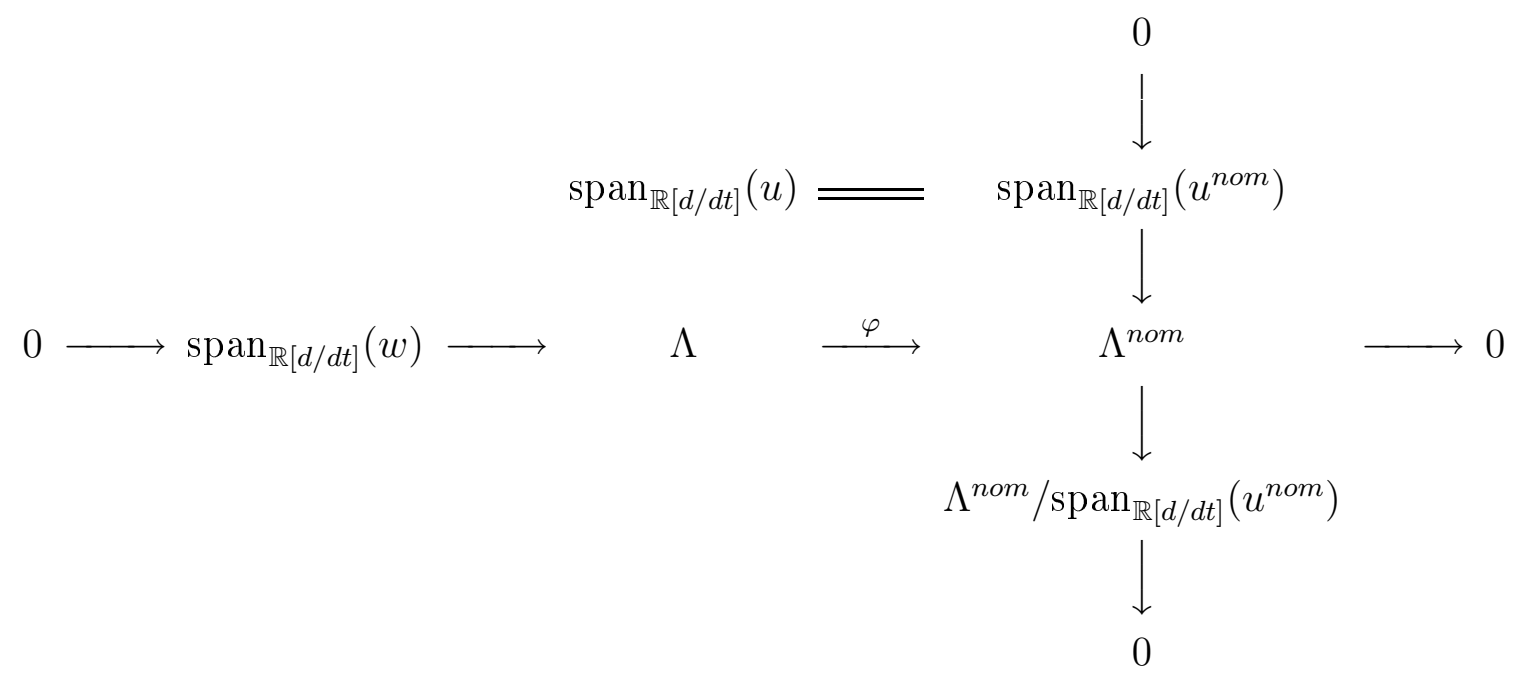

$\operatorname{avec} \Lambda^{\text {nom }}=\Lambda / \operatorname{span}_{\mathbb{R}[d / d t]}(w)$.

La perturbation ne joue aucun rôle dans la définition tant de la commandabilité que de l'observabilité. Ainsi, nous dirons qu'un système (5.4) est commandable ou observable si, et seulement si, $\Lambda^{\text {nom }}=\Lambda / \operatorname{span}_{\mathbb{R}[d / d t]}(w)$ est commandable ou observable.

Définition 5.1 Le système (5.4) est commandable si, et seulement si, $\Lambda^{\text {nom }}$ (qui lui est associé) est libre.

Suite à cette définition, nous remarquons que si le système (5.4) est commandable, le module nominal $\Lambda^{\text {nom }}$ qui lui est associé ne posséde pas d'éléments de torsion.

Avec une écriture de système kalmanienne, s'il est possible d'écrire ce qui suit après un changement de coordonnées :

$$
\Sigma_{L}:\left\{\begin{array}{l}
\vdots \\
\dot{\tilde{x}}_{i}=\alpha_{i} \tilde{x}_{i} \quad, \quad w=0 \\
\vdots
\end{array}\right.
$$

il est trivial de constater que cette partie de l'état n'est pas commandable.

En algèbre ceci s'exprime ainsi $\left(\operatorname{avec} \tilde{x}_{i}(t) \neq 0\right)$ : il existe un élément $a$ de l'anneau $k[d / d t]$ tel que $a \tilde{x}_{i}=0$. Dans ce cas $a=d / d t-\alpha_{i}$ et il existe alors au moins un élément de torsion. Ainsi, $\Lambda^{\text {nom }}$ n'est pas libre ce qui signifie d'après la définition que le système n'est pas commandable.

Définition 5.2 Tout m-uplet d'éléments de $\Lambda$, dont l'image canonique dans $\Lambda^{\text {nom }}$ est une base, est appelé sortie plate.

Les sorties plates jouent un rôle d'importance dans la commande des systèmes et plus particulièrement dans la commande des systèmes non linéaires en boucle ouverte ([21]). 
Nous accordons un intérêt particulier à la définition qui suit car elle est très proche des définitions analytiques proposées dans les chapitres précédents ( $c f . y$-reconstructabilité définition 2.4).

Définition 5.3 Le système (5.4) est observable si, et seulement si, $\Lambda^{\text {nom }}$ et $\operatorname{span}_{\mathbb{R}[d / d t]}\left(u^{\text {nom }}, y^{\text {nom }}\right)$ coïncident.

Nous notons avec quelle facilité l'ensemble des signaux dits connus sont représentés à l'aide de la théorie des modules $\left(\operatorname{span}_{\mathbb{R}[d / d t]}\left(u^{n o m}, y^{n o m}\right)\right)$. Nous ne manquons pas de faire le parallèle avec ce que nous développons dans le chapitre 2 et les difficultés rencontrées en terme de formalisme.

Dans la section suivante, nous proposons un formalisme algébrique de notions bien connues en diagnostic de systèmes. Ainsi nous nous proposons de revenir sur les définitions des chapitres précédents : détectabilité, isolabilité et découplage total à l'aide de la théorie des modules.

\subsection{Diagnostic des systèmes linéaires : une approche al- gébrique}

Lors de l'étude d'un système menant à son diagnostic, la première étape est sans doute de vérifier si l'ensemble des défauts considérés sont détectables. Il s'agit de l'objet du paragraphe suivant.

\subsubsection{Détectabilité des défauts}

Afin d'appréhender au mieux la notion de détectabilité en terme algébrique, nous étudions dans un premier temps un cas particulier. Puis nous étendons la définition qui en découle au cas général.

\subsubsection{Etude du cas mono-défaut}

Nous supposons que seul un défaut peut apparaître sur le système $(\operatorname{dim}(w)=1)$.

Un défaut est dit détectable si, et seulement si, il a un effet sur l'une au moins des sorties. En d'autres termes, lors de l'écriture des sorties et/ou de leurs dérivées, il doit apparaître l'expression du défaut $w$ et/ou de ses dérivées.

A l'aide des modules cette propriété peut se formaliser comme suit, avec $\Gamma_{u, w, y} \subseteq \Lambda$ le $\mathbb{R}[d / d t]$-module engendré par $\operatorname{span}_{\mathbb{R}[d / d t]}(u, w, y)$ : 
Définition $5.4 S i \Gamma_{u, w, y} / \operatorname{span}_{\mathbb{R}[d / d t]}(u)$ :

- est de torsion $\Leftrightarrow$ le défaut est dit non détectable,

- n'est pas de torsion $\Leftrightarrow$ le défaut est dit détectable.

Nous insistons sur le dernier point, car toutes les sorties ne sont pas nécessairement sensibles au défaut. En effet, en ajoutant que si le module n'est pas de torsion, cela ne signifie pas qu'il est libre, il suffit que la partie libre de $\Gamma_{u, w, y} / \operatorname{span}_{\mathbb{R}[d / d t]}(u)$ ne soit pas réduite à l'ensemble $\{0\}$.

De plus cette définition est basée sur l'indépendance de la commande et du défaut $\left(\operatorname{span}_{\mathbb{R}[d / d t]}(u) \cap \operatorname{span}_{\mathbb{R}[d / d t]}(w)=\{0\}\right)$.

Puisqu'il s'agit de l'étude d'un cas particulier (mono-défaut), nous étendons cette étude au cas plus général (multi-défauts).

\subsubsection{Etude du cas multi-défauts}

Plusieurs défauts peuvent apparaître sur le système $(\operatorname{dim}(w) \geq 1)$.

Les défauts sont détectables seulement si chaque signal scalaire $w_{i}$ l'est. En effet, si $\Gamma_{u, w, y} / \operatorname{span}_{\mathbb{R}[d / d t]}(u)$ n'est pas de torsion, alors une partie du défaut affecte les sorties mais rien ne prouve que tous les défauts agissent sur au moins l'une des sorties. Rappelons le cas où une partie du défaut est naturellement découplée et appartient au sous-espace d'état inobservable.

Définition 5.5 Tous les défauts sont dits détectables si, et seulement si, $\Gamma_{u, w, y} / \operatorname{span}_{\mathbb{R}[d / d t]}\left(u, \underline{w_{i}}\right)$ n'est pas de torsion avec $\underline{w_{i}}=\left[w_{1}, \ldots, w_{i-1}, w_{i+1}, \ldots, w_{q}\right]$ et $i=\{1, \cdots, q\}$.

Nous proposons le théorème suivant qui lie les définitions 5.4 et 5.5.

Théorème 5.1 Avec $\operatorname{dim}(w) \geq 1$, si $\Gamma_{u, w, y} / \operatorname{span}_{\mathbb{R}[d / d t]}(u)$ n'est pas de torsion alors l'une au moins des composantes du vecteur défaut est détectable.

\section{Preuve}

Si $\Gamma_{u, w, y} / \operatorname{span}_{\mathbb{R}[d / d t]}(u)$ n'est pas de torsion alors il existe un sous-module de $\Gamma_{u, w, y} / \operatorname{span}_{\mathbb{R}[d / d t]}(u)$ libre non réduit à $\{0\}$. Or seule l'apparition de $w$ au sein de ce sousmodule assure la non réduction à $\{0\}$ de sa partie libre car $\Gamma_{u, w, y} / \operatorname{span}_{\mathbb{R}[d / d t]}(u, w)$ est toujours un module de torsion. Alors il existe $i$ tel que $\Gamma_{u, w, y} / \operatorname{span}_{\mathbb{R}[d / d t]}\left(u, \underline{w_{i}}\right)$ ne soit pas de torsion. D'après la définition 5.5 ceci signifie qu'au moins la composante $w_{i}$ du défaut est détectable. 
Lors de l'étude d'un système pour le diagnostic, suite à l'établissement de la détectabilité de l'ensemble des défauts, l'automaticien souhaite les distinguer. Le paragraphe suivant propose une définition de cette propriété.

\subsubsection{Isolabilité des défauts}

Pour débuter l'étude de l'isolabilité des défauts, nous exprimons cette notion à l'aide de la phrase suivante :

un ensemble de défauts est isolable si à partir des signaux connus (sorties $y$ et commandes $u$ ) il est possible de les distinguer.

Dans ce qui suit, nous considèrons un cas d'isolabilité beaucoup plus large que ce que nous énonçons dans le chapitre 2 en début de section 2.4 ( $c f$. géométriquement isolable), car dans ce qui suit la dynamique des défauts sur les sorties est considérée et peut être un moyen de distinction.

L'hypothèse générale $\operatorname{span}_{\mathbb{R}[d / d t]}\left(w_{i}\right), \forall i=\{1, \cdots, q\}$ est sans torsion, implique que le nombre de sorties doit nécessairement être supérieur ou égal au nombre de défauts à isoler $(\operatorname{dim}(y) \geq \operatorname{dim}(w)=q)$.

Nous présentons dans un premier temps le cas général, puis à l'aide de l'étude du cas particulier 2_sorties-2_défauts, nous illustrons la méthode algébrique proposée.

\subsubsection{Etude du cas général}

Nous considérons un système possédant $p$ sorties et $q$ défauts $(p \geq q)$. A chaque défaut $w_{i}$ est associé le calcul d'un sous-module de $\Lambda$ :

$$
V_{i}=\operatorname{span}_{\mathbb{R}[d / d t]}\left(w_{i}\right) \cap \operatorname{span}_{\mathbb{R}[d / d t]}(y, u)
$$

Définition 5.6 L'ensemble des défauts sont dit isolables si, et seulement si :

$$
V_{i}=\operatorname{span}_{\mathbb{R}[d / d t]}\left(w_{i}\right) \cap \operatorname{span}_{\mathbb{R}[d / d t]}(y, u) \neq\{0\}, \forall i
$$

En remarque, nous ajoutons que l'hypothèse de détectabilité n'est pas nécessaire. En effet, l'énoncé de la définition précédente inclut cette hypothèse car il y a intersection entre les deux sous-modules uniquement lorsque le défaut possède un effet sur l'une au moins des sorties (il s'agit de la définition de la détectabilité).

D'après cette remarque, le théorème suivant semble trivial :

Théorème 5.2 Si l'ensemble des défauts sont isolables alors ils sont détectables. 


\section{Preuve}

En considérant la définition de l'isolabilité d'un défaut $w_{i}$ qui est $V_{i}=\operatorname{span}_{\mathbb{R}[d / d t]}\left(w_{i}\right) \cap$ $\operatorname{span}_{\mathbb{R}[d / d t]}(y, u) \neq\{0\}$, celle-ci permet de qualifier le module $\operatorname{span}_{\mathbb{R}[d / d t]}\left(y, u, w_{i}\right) / \operatorname{span}_{\mathbb{R}[d / d t]}(u)$ comme n'étant pas un module de torsion. Il semble alors évident que $\operatorname{span}_{\mathbb{R}[d / d t]}(y, u, w) / \operatorname{span}_{\mathbb{R}[d / d t]}\left(u, \underline{w_{i}}\right)$ en rappelant que $\underline{w_{i}}=\left(\begin{array}{lllllll}w_{1} & \cdots & w_{i-1} & w_{i+1} & \cdots & w_{q}\end{array}\right)$ n'est pas un module de torsion (définition de la détectabilité).

Afin de réaliser un lien entre les notions introduites dans ce chapitre et celles régulièrement employées dans la littérature, nous proposons le théorème suivant :

Théorème 5.3 Si l'ensemble des q défauts sont isolables $\Leftrightarrow$ la matrice de transfert des défauts sur les sorties est de rang $q$.

\section{Preuve}

$(\Leftarrow)$ si la matrice de transfert des défauts sur les sorties est de rang q (en rappelant que $q \leq p$ ) alors il est possible de l'écrire comme suit (via un changement de coordonnées) :

$$
\tilde{T}_{y w}=U(s)\left(\begin{array}{cccc}
\tilde{c}_{1}(s) & 0 & \cdots & 0 \\
0 & \ddots & \ddots & \vdots \\
\vdots & \ddots & \ddots & 0 \\
0 & \cdots & 0 & \tilde{c}_{q}(s) \\
0 & \cdots & \cdots & 0 \\
\vdots & & & \vdots \\
0 & \cdots & \cdots & 0
\end{array}\right)
$$

où s est l'opérateur de Laplace.

Ceci implique une transformation des sorties de la manière suivante :

$$
\left\{\begin{array} { l } 
{ a _ { 1 } ( s ) y _ { 1 } = b _ { 1 } ( s ) u + c _ { 1 } ( s ) w _ { 1 } + \cdots + d _ { 1 } ( s ) w _ { q } } \\
{ \vdots } \\
{ a _ { q } ( s ) y _ { q } = b _ { q } ( s ) u + c _ { q } ( s ) w _ { 1 } + \cdots + d _ { q } ( s ) w _ { q } } \\
{ a _ { q + 1 } ( s ) y _ { q + 1 } = b _ { q + 1 } ( s ) u + c _ { q + 1 } ( s ) w _ { 1 } + \cdots + d _ { q + 1 } ( s ) w _ { q } } \\
{ \vdots } \\
{ a _ { p } ( s ) y _ { p } = b _ { p } ( s ) u + c _ { p } ( s ) w _ { 1 } + \cdots + d _ { p } ( s ) w _ { q } }
\end{array} \Longleftrightarrow \left\{\begin{array}{l}
\bar{a}_{1}(s) y-\bar{b}_{1}(s) u=\bar{c}_{1}(s) w_{1} \\
\vdots \\
\bar{a}_{q}(s) y-\bar{b}_{q}(s) u=\bar{c}_{q}(s) w_{q} \\
\bar{a}_{q+1}(s) y-\bar{b}_{q+1}(s) u=0 \\
\vdots \\
\bar{a}_{p}(s) y-\bar{b}_{p}(s) u=0
\end{array}\right.\right.
$$

Il est alors trivial que $V_{i}=\operatorname{span}_{\mathbb{R}[d / d t]}\left(w_{i}\right) \cap \operatorname{span}_{\mathbb{R}[d / d t]}(y, u) \neq\{0\}$ avec $i=\{1, \cdots, q\}$, les défauts sont donc isolables par définition. 
$(\Rightarrow)$ si les défauts sont isolables alors chaque sous-module $V_{i}=\operatorname{span}_{\mathbb{R}[d / d t]}\left(w_{i}\right) \cap \operatorname{span}_{\mathbb{R}[d / d t]}(y, u) \neq$

$\{0\}$ ainsi à chaque défaut, il est possible d'associer l'équation suivante :

$$
\left\{\begin{array}{l}
p_{1}(s) w_{1}=q_{1}(s) y_{1}+\cdots+r_{1}(s) y_{p}+s_{1}(s) u \\
\vdots \\
r_{q}(s) w_{q}=q_{q}(s) y_{1}+\cdots+r_{q}(s) y_{p}+s_{q}(s) u
\end{array}\right.
$$

A l'aide d'une transformation équivalente à la précédente nous assurons l'écriture suivante:

$$
\left\{\begin{array}{l}
\bar{q}_{1}(s) \bar{y}_{1}=\bar{s}_{1}(s) u-\left(\bar{p}_{1}(s) w_{1}+\cdots+\bar{q}_{1}(s) w_{q}\right) \\
\vdots \\
\bar{q}_{q}(s) \bar{y}_{q}=\bar{s}_{q}(s) u-\left(\bar{p}_{q}(s) w_{1}+\cdots+\bar{q}_{q}(s) w_{q}\right)
\end{array}\right.
$$

Le problème lié à cette équation est l'apparition de combinaison de sorties dans le membre de gauche. Mais ajouter que $\bar{y}=H(s)$ y avec $H(s)$ inversible (de rang q) permet de conclure.

Dans le cadre où les défauts ne sont pas isolables, il semble particulièrement intéressant d'étudier l'intersection $\operatorname{span}_{\mathbb{R}[d / d t]}\left(w_{i}\right) \cap \operatorname{span}_{\mathbb{R}[d / d t]}\left(y, u, w_{j}\right)$ avec $i \neq j$. Cette étude permet l'isolation d'un groupe de défauts. Par exemple,

$$
\begin{aligned}
& \operatorname{span}_{\mathbb{R}[d / d t]}\left(w_{1}\right) \cap \operatorname{span}_{\mathbb{R}[d / d t]}(y, u)=\{0\} \\
& \operatorname{span}_{\mathbb{R}[d / d t]}\left(w_{2}\right) \cap \operatorname{span}_{\mathbb{R}[d / d t]}\left(y, u, w_{1}\right) \neq\{0\} \\
& \operatorname{span}_{\mathbb{R}[d / d t]}\left(w_{3}\right) \cap \operatorname{span}_{\mathbb{R}[d / d t]}\left(y, u, w_{1}\right) \neq\{0\}
\end{aligned}
$$

dans cet exemple, les défauts $w_{2}$ et $w_{3}$ sont isolables de par leurs effets sur les sorties.

Le paragraphe suivant présente les résultats dans le cadre de l'étude d'un système possédant 2 sorties et 2 défauts.

\subsubsection{Etude du cas particulier 2_sorties-2_défauts}

Considérons que $\operatorname{dim}(w)=\operatorname{dim}(y)=p=q=2$, c'est-à-dire :

$$
\Sigma_{L}:\left\{\begin{array}{l}
\dot{x}=A x+B u+P w \\
y=C x
\end{array} \Longleftrightarrow \Sigma_{L}:\left\{\begin{array}{l}
\dot{x}=A x+B u+P_{1} w_{1}+P_{2} w_{2} \\
y_{1}=C_{1} x \\
y_{2}=C_{2} x
\end{array}\right.\right.
$$

D'après la définition précédente du système, il est toujours possible d'écrire les sorties de la manière suivante :

$$
\begin{aligned}
& a_{1}(s) y_{1}=b_{1}(s) u+c_{1}(s) w_{1}+d_{1}(s) w_{2} \\
& a_{2}(s) y_{2}=b_{2}(s) u+c_{2}(s) w_{1}+d_{2}(s) w_{2}
\end{aligned}
$$


avec $a_{i}(s), b_{i}(s), c_{i}(s), d_{i}(s)$, des polynômes en $s$ (ce sont donc des éléments de l'anneau $\mathbb{R}[d / d t])$.

Nous proposons une étude de cas qui permettra une meilleure appréhension du problème. A cette fin, nous définissons la matrice de transfert des défauts sur les sorties comme suit :

$$
T_{y w}=\left(\begin{array}{ll}
c_{1}(s) & d_{1}(s) \\
c_{2}(s) & d_{2}(s)
\end{array}\right)
$$

Nous ajoutons (sous l'hypothèse de détectabilité de tous les défauts) que l'un au moins des polynômes $c_{1}(s)$ (resp. $d_{1}(s)$ ) et $c_{2}(s)$ (resp. $d_{2}(s)$ ) est non nul, c'est-à-dire qu'il ne peut $\mathrm{y}$ avoir plus de deux zéros dans la matrice $T_{y w}$. Pour débuter l'étude, nous énumérons les cas triviaux :

- dans le cas où seule une composante de la matrice $T_{y w}$ est nulle alors les défauts sont isolables,

- dans le cas où deux composantes de la matrice $T_{y w}$ sont nulles alors les défauts sont isolables (resp. non isolables) si les zéros ne sont pas sur la même ligne (resp. sont sur la même ligne).

Par conséquent seul le cas où les quatre polynômes sont non nulles est à étudier.

Il faut alors se poser la question suivante :

"Est-il possible d'éliminer, par substitution, l'effet d'un défaut sur l'une des deux sorties ?"

La réponse est "oui", si les équations ne sont pas équivalentes à un polynôme multiplicateur prêt, c'est-à-dire $\left.\left.p_{1}(s)\left(c_{1}(s) w_{1}+d_{1}(s) w_{2}\right)\right) \neq p_{2}(s)\left(c_{2}(s) w_{1}+d_{2}(s) w_{2}\right)\right)$, pour tous les couples de polynômes $\left(p_{1}(s), p_{2}(s)\right) \neq(0,0)$. Dans le cas contraire (égalité), il existe un transfert liant les deux équations de sorties $\left(p_{1}(s) p_{2}^{-1}(s)\right)$, ce qui interdit toutes possibilités de substitutions.

Sous l'hypothèse d'inégalité, les deux équations ((5.17) et (5.18)) peuvent s'écrire :

$$
\begin{aligned}
& c_{2}(s) a_{1}(s) y_{1}=c_{2}(s) b_{1}(s) u+c_{2}(s) c_{1}(s) w_{1}+c_{2}(s) d_{1}(s) w_{2} \\
& c_{1}(s) a_{2}(s) y_{2}=c_{1}(s) b_{2}(s) u+c_{1}(s) c_{2}(s) w_{1}+c_{1}(s) d_{2}(s) w_{2}
\end{aligned}
$$

et permettent :

$$
c_{2}(s) a_{1}(s) y_{1}-c_{1}(s) a_{2}(s) y_{2}-\left(c_{2}(s) b_{1}(s)-c_{1}(s) b_{2}(s)\right) u=\left(c_{2}(s) d_{1}(s)-c_{1}(s) d_{2}(s)\right) w_{2}
$$

Il existe alors une dynamique de $w_{2}$ (resp. $w_{1}$ ) exprimable à l'aide des sorties et de la commande. Dans ce cas, en considérant le corps rationnel, nous écrivons :

$$
\begin{aligned}
& \left(c_{2}(s) d_{1}(s)-c_{1}(s) d_{2}(s)\right)^{-1} c_{2}(s) a_{1}(s) y_{1} \\
& -\left(c_{2}(s) d_{1}(s)-c_{1}(s) d_{2}(s)\right)^{-1} c_{1}(s) a_{2}(s) y_{2} \\
& -\left(c_{2}(s) d_{1}(s)-c_{1}(s) d_{2}(s)\right)^{-1}\left(c_{2}(s) b_{1}(s)-c_{1}(s) b_{2}(s)\right) u=w_{2}
\end{aligned}
$$


Il semble alors trivial d'exprimer une combinaison de sorties et de commande seulement affectées par le défaut $w_{1}$ :

$$
\begin{aligned}
a_{1}(s) y_{1}= & \\
& d_{1}(s)\left(c_{2}(s) d_{1}(s)-c_{1}(s) d_{2}(s)\right)^{-1} c_{2}(s) a_{1}(s) y_{1} \\
& -d_{1}(s)\left(c_{2}(s) d_{1}(s)-c_{1}(s) d_{2}(s)\right)^{-1} c_{1}(s) a_{2}(s) y_{2} \\
& -d_{1}(s)\left(c_{2}(s) d_{1}(s)-c_{1}(s) d_{2}(s)\right)^{-1}\left(c_{2}(s) b_{1}(s)-c_{1}(s) b_{2}(s)\right) u \\
& +b_{1}(s) u+c_{1}(s) w_{1}
\end{aligned}
$$

En conclusion des calculs précédents, nous savons que l'équation (5.23) (resp. (5.22)) permet l'isolation du défaut $w_{1}$ (resp. $w_{2}$ ) puisqu'elle ne fait intervenir que son écriture et non celle de $w_{2}$ (resp. $\left.w_{1}\right)$.

Ainsi une manière subjective d'appréhender les conditions d'isolation de défauts est de considérer que s'il existe une combinaison de sorties et commandes permettant d'écrire chacun des défauts indépendamment les uns des autres alors ils sont isolables.

Dans ce qui suit, nous proposons, dans les mêmes conditions particulières $(p=q=2)$, l'énoncé des conditions d'isolation au moyen de la théorie des modules. Cependant comme nous venons de l'illustrer à l'aide d'une pré-étude, les fonctions de transfert sont nécessaires. Nous introduisons $\mathbb{R}(d / d t)$ le corps de fraction de $\mathbb{R}[d / d t]$. Soit le $\mathbb{R}(d / d t)$-espace vectoriel appelé espace de transfert du système $\Lambda$ défini comme suit :

$$
\hat{\Lambda}=\mathbb{R}(d / d t) \otimes_{\mathbb{R}[d / d t]} \Lambda
$$

où $\mathbb{R}(d / d t) \otimes_{\mathbb{R}[d / d t]} \bullet$ est le foncteur de localisation ( $c f$. annexe $\mathrm{C}$ ). Il semble important de rappeler que le foncteur de Laplace n'est pas fidèle. Cependant, par définition nous considérons que tout état est, soit influencé par un défaut, soit influencé par la commande. Ceci assure l'absence de tout élément de torsion dans $\Lambda$ et donc la réduction à $\{0\}$ de $\Lambda_{\text {torsion }}$. Evidemment, l'égalité $\Lambda_{\text {torsion }}=\{0\}$ n'est en rien synonyme de commandabilité, notion établie à partir de l'étude de $\Lambda^{\text {nom }}=\Lambda / \operatorname{span}_{\mathbb{R}[d / d t]}(w)$.

Posons :

$$
\begin{aligned}
& V_{1}=\operatorname{span}_{\mathbb{R}[d / d t]}\left(w_{1}\right) \cap \operatorname{span}_{\mathbb{R}[d / d t]}\left(y_{1}, y_{2}, u\right) \\
& V_{2}=\operatorname{span}_{\mathbb{R}[d / d t]}\left(w_{2}\right) \cap \operatorname{span}_{\mathbb{R}[d / d t]}\left(y_{1}, y_{2}, u\right)
\end{aligned}
$$

Nous rappelons que par définition, $w$ est une entrée exogène ce qui assure $\operatorname{span}_{\mathbb{R}[d / d t]}(u) \cap$ $\operatorname{span}_{\mathbb{R}[d / d t]}(w)=\{0\}$. Ainsi, nous interprétons l'existence (non réduction à $\{0\}$ ) du sousmodule $V_{1}$ (resp. $V_{2}$ ) par l'existence d'une équation du type (5.23) (resp. (5.22)) c'est-à-dire $p_{1}(s) y_{1}+p_{2}(s) y_{2}+p_{3}(s) u=p_{4}(s) w_{1}$ (resp. $\left.p_{1}(s) y_{1}+p_{2}(s) y_{2}+p_{3}(s) u=p_{4}(s) w_{2}\right)$. Ceci est une conséquence directe de la $\mathbb{R}(s)$-linéaire indépendance de $y_{1}$ et $y_{2}$.

En conclusion, les défauts sont isolables si, et seulement si, chaque sous-module associé 
à chaque défaut est non nul $\left(V_{i} \neq\{0\}, \forall i\right)$.

Notons que la définition de l'isolation des défauts proposée est directement liée à leur estimabilité (chapitre 2). Il parait naturel suite à ces définitions de définir le découplage total en terme algébrique.

\subsubsection{Système totalement découplable}

En considérant les signaux connus $u$ et $y$, à $\operatorname{span}_{\mathbb{R}[d / d t]}(u, y)$ nous associons l'ensemble des signaux $\Delta$-reconstructibles.

Ainsi la définition du découplage total est la suivante :

Définition 5.7 Un système linéaire $\Lambda$ de commande u et sortie y perturbé est dit totalement découplable si, et seulement si, $\operatorname{span}_{\mathbb{R}[d / d t]}(w) \subseteq \operatorname{span}_{\mathbb{R}[d / d t]}(u, y)$

Cette définition est justifiée quelle que soit la dimension de $w$ (cas mono et multi-défauts).

Nous ajoutons en remarque que dans le cadre des systèmes observables ceci signifie que $\Lambda$ et $\operatorname{span}_{\mathbb{R}[d / d t]}(u, y)$ coïncident.

\subsection{Exemples illustratifs et synthèse de filtres}

Dans ce paragraphe, nous proposons l'étude de plusieurs modèles académiques fictifs afin d'appliquer et d'illustrer les différentes notions précédemment introduites.

\subsubsection{Exemple 1}

$$
\Sigma_{L}:\left\{\begin{array}{l}
\dot{x}_{1}=-x_{1}+w_{1} \\
\dot{x}_{2}=-x_{2}+w_{2} \\
y=x_{1}
\end{array}\right.
$$

Nous débutons l'étude de cet exemple par l'évaluation de la détection de chacun des défauts. Ainsi d'après la définition 5.5, les modules $\operatorname{span}_{\mathbb{R}[d / d t]}(y, u, w) / \operatorname{span}_{\mathbb{R}[d / d t]}\left(u, w_{2}\right)$ et $\operatorname{span}_{\mathbb{R}[d / d t]}(y, u, w) / \operatorname{span}_{\mathbb{R}[d / d t]}\left(u, w_{1}\right)$ sont à étudier afin de déterminer s'il s'agit de modules de torsion.

Or avec $\dot{y}=\dot{x}_{1}=-x_{1}+w_{1}, y^{(i)}=(-1)^{i} x_{1}+w_{1}^{(i-1)}-w_{1}^{(i-2)} \cdots(-1)^{i-1} w_{1}$, $\operatorname{span}_{\mathbb{R}[d / d t]}(y, u, w) / \operatorname{span}_{\mathbb{R}[d / d t]}\left(u, w_{2}\right)$ n'est pas de torsion et donc $w_{1}$ est détectable. 
En revanche, $\operatorname{span}_{\mathbb{R}[d / d t]}(y, u, w) / \operatorname{span}_{\mathbb{R}[d / d t]}\left(u, w_{1}\right)$ est un sous-module de torsion car : avec $y \neq 0$, il existe $P(s) \neq 0$ tel que $P(s) y=0$, par exemple $P(s)=(s+1)$ et tous les polynômes $\mathbb{R}(s)$-linéaires dépendants. Alors le défaut $w_{2}$ n'est pas détectable.

De part l'établissement de la non détectabilité du défaut $w_{2}$, l'isolabilité ne peut être prouvée.

\subsubsection{Exemple 2}

$$
\Sigma_{L}:\left\{\begin{array}{l}
\dot{x}_{1}=-x_{1}+w_{1} \\
\dot{x}_{2}=-x_{2}+w_{2} \\
\dot{x}_{3}=-x_{1}+x_{2}-x_{3} \\
y_{1}=x_{1}+x_{2} \\
y_{2}=x_{3}
\end{array}\right.
$$

avec la seule écriture $\dot{y}_{1}=-x_{1}-x_{2}+w_{1}+w_{2}$, nous prouvons que les défauts $w_{1}$ et $w_{2}$ sont détectables.

Afin d'établir l'isolabilité des défauts, nous nous référons à la définition 5.6. C'est-à-dire que nous recherchons d'éventuelles intersections $\operatorname{span}_{\mathbb{R}[d / d t]}\left(w_{1}\right) \cap \operatorname{span}_{\mathbb{R}[d / d t]}(y, u)$ et $\operatorname{span}_{\mathbb{R}[d / d t]}\left(w_{2}\right) \cap$ $\operatorname{span}_{\mathbb{R}[d / d t]}(y, u)$ non réduites à l'espace nul.

Or, sans détailler les calculs, il est possible de déterminer les équations suivantes :

$$
\begin{gathered}
(s+1) y_{1}=w_{1}+w_{2} \\
\left(s^{2}+2 s+1\right) y_{2}=-w_{1}+w_{2}
\end{gathered}
$$

ainsi, nous obtenons $2 * w_{1}=(s+1) y_{1}-\left(s^{2}+2 s+1\right) y_{2}$ et $2 * w_{2}=(s+1) y_{1}+\left(s^{2}+2 s+1\right) y_{2}$ ce qui permet d'assurer respectivement que $V_{1}=\operatorname{span}_{\mathbb{R}[d / d t]}\left(w_{1}\right) \cap \operatorname{span}_{\mathbb{R}[d / d t]}(y, u) \neq\{0\}$ et $V_{2}=\operatorname{span}_{\mathbb{R}[d / d t]}\left(w_{2}\right) \cap \operatorname{span}_{\mathbb{R}[d / d t]}(y, u) \neq\{0\}$. Puisque $V_{1}$ et $V_{2}$ sont non réduits à l'espace nul alors les deux défauts $w_{1}$ et $w_{2}$ sont isolables.

De même, en référence à la méthode proposée dans la section 3.2.2 nous synthétisons les filtres suivant :

$$
\Sigma_{F D 1}:\left\{\begin{array}{l}
\dot{z}_{1}=-z_{1}+\Psi_{1} \\
\dot{z}_{2}=-z_{2} \\
\dot{z}_{3}=z_{1}+z_{2}+z_{3} \\
y_{z 1}=z_{1}+z_{2} \\
y_{z 2}=z_{3}
\end{array} \quad \Sigma_{F D 2}:\left\{\begin{array}{l}
\dot{z}_{1}=-z_{1} \\
\dot{z}_{2}=-z_{2}+\Psi_{2} \\
\dot{z}_{3}=z_{1}+z_{2}+z_{3} \\
y_{z 1}=z_{1}+z_{2} \\
y_{z 2}=z_{3}
\end{array}\right.\right.
$$


avec les égalités suivantes : $\Psi_{1}=1 / 2\left((s+1) y_{1}-\left(s^{2}+2 s+1\right) y_{2}\right)$ et $\Psi_{2}=1 / 2\left((s+1) y_{1}+\left(s^{2}+2 s+1\right) y_{2}\right)$. En remarque, nous insistons sur le fait que dans ce cas $\Psi_{\bullet} \subset \operatorname{span}_{\mathbb{R}[d / d t]}(y)$, mais il faut préciser que d'une manière générale, $\Psi_{T \bullet} \subset \hat{\Lambda}$ avec $\Psi_{\bullet}=\Psi_{T \bullet}(y u)$.

Nous assurons l'insensibilité du résidu $r_{1}=y_{x}-y_{z}$ (resp. $r_{2}=y_{x}-y_{z}$ ) au défaut $w_{2}$ (resp. $\left.w_{1}\right)$ c'est-à-dire que $r_{1}=0, \forall w_{1}\left(\operatorname{resp} . r_{2}=0, \forall w_{2}\right)$ et $r_{1} \neq 0, w_{2} \neq 0\left(\operatorname{resp} . r_{2} \neq 0, w_{1} \neq 0\right)$.

\subsubsection{Exemple 3}

$$
\Sigma_{L}:\left\{\begin{array}{l}
\dot{x}_{1}=-x_{1}+w_{1} \\
\dot{x}_{2}=-x_{1}-x_{2}+w_{2} \\
\dot{x}_{3}=-x_{2}-x_{3} \\
y_{1}=x_{2} \\
y_{2}=x_{3}
\end{array}\right.
$$

Il semble évident que les défauts sont détectables.

A l'aide d'une approche similaire à celle proposée dans l'exemple précédent, nous recherchons d'éventuelles intersections $\operatorname{span}_{\mathbb{R}[d / d t]}\left(w_{1}\right) \cap \operatorname{span}_{\mathbb{R}[d / d t]}(y, u)$ et $\operatorname{span}_{\mathbb{R}[d / d t]}\left(w_{2}\right) \cap \operatorname{span}_{\mathbb{R}[d / d t]}(y, u)$ étant non réduites à l'espace nul.

Or, il s'avère que :

$$
\begin{gathered}
\left(s^{2}+2 s+1\right) y_{1}=-w_{1}+(s+1) w_{2} \\
\left(s^{3}+3 s^{2}+3 s+1\right) y_{2}=w_{1}-(s+1) w_{2}
\end{gathered}
$$

et ces deux équations ne sont pas $\mathbb{R}(s)$-linéaires indépendantes. Ainsi nous concluons l'étude en ajoutant qu'il n'est pas possible d'exprimer les défauts indépendamment à l'aide des sorties et commandes $\left(V_{1}=V_{2}=\{0\}\right)$. Les défauts ne sont donc pas isolables.

\subsection{Conclusion}

Dans ce chapitre, sont proposées des définitions algébriques de propriétés qualifiant des systèmes L.T.I. (ou L.T.V.). Ces définitions générales sont indépendantes du type de représentation d'état utilisée (kalmanienne ou généralisée). De plus, il semble également ([24]) que l'approche algébrique soit bien appropriée pour représenter les systèmes à retard.

Ce chapitre d'ouverture offre des perspectives qui nous paraissent intéressantes dans l'étude des systèmes non linéaires. En effet, à première vue, la seule définition de nouveaux opérateurs sur l'anneau permettrait la modèlisation des systèmes non linéaires. Les définitions précédentes de la détectabilité et l'isolabilité, objets des chapitres 1 et 2 de ce manuscrit, 
seraient encore valides.

Cependant, cette formulation est à relativiser car il ne faut en aucun cas sous-évaluer la difficulté que représente l'étude des systèmes non linéaires. 


\section{Conclusion générale et perspectives}

Le travail présenté dans ce mémoire porte sur le diagnostic des systèmes non linéaires qui ne peut pas s'établir à l'aide des différentes méthodes classiques développées dans un cadre linéaire. Nous établissons avec précision les différentes étapes nécessaires à l'isolation des défauts en contribuant à leurs améliorations.

Dans un premier temps, les méthodes récentes de découplage de l'état aux perturbations sont rappelées. Nous comparons ces méthodes afin d'en faire ressortir les différents avantages et inconvénients. Grâce au découplage, nous attribuons aux résidus différentes caractéristiques de sensibilité qui nous permettent de différencier les défauts. Ainsi plusieurs schémas correspondant à différentes sensibilités sont présentés.

Dans un deuxième temps, nous évaluons une méthode de découplage en étudiant la dimension du sous-espace d'état rendu insensible à la perturbation via une injection de sortie. En nous fondant sur l'écriture d'une injection de sortie générale, nous améliorons les méthodes de découplage en augmentant la dimension du sous-espace d'état insensible à une perturbation.

Sous l'hypothèse de non apparition simultanée des défauts, les conditions suffisantes à leur détection et isolation sont déclinées. Suite à une analyse des propriétés géométriques du sousespace d'état associé à chaque défaut, des conditions moins restrictives que celles résolvant le problème fondamental de la génération de résidus sont obtenues. En effet, dans le cadre de notre approche, un résidu n'est plus exclusivement sensible à un unique défaut.

Sous l'hypothèse que les conditions d'isolation des défauts sont satisfaites, nous développons une méthode systématique de synthèse de filtres en accord avec l'analyse théorique du modèle du système. Puisque les conditions d'invariance à la dynamique du système ne peuvent pas toujours être satisfaites à l'aide d'un unique filtre, différentes structures de filtres sont exposées.

De plus, l'étude de la convergence est importante dans tout problème d'estimation d'état ou de génération de résidus. En nous appuyant sur les résultats novateurs obtenus par analyse de la contraction, la synthèse de l'injection de sortie est modifiée. L'application de la théorie de la contraction assure la convergence exponentielle du résidu vers zéro lorsque les états 
initiaux du filtre et du système s'avèrent différents.

L'ensemble de ces propositions est appliqué au Benchmark des trois cuves. Les résultats obtenus avec des méthodes antérieures sont tout d'abord comparés d'un point de vue théorique avec nos propositions avant d'être illustrés à l'aide de simulations.

Enfin, nous détaillons avec précision une première perspective que nous envisageons à ces travaux. En effet, les travaux réalisés dans ce mémoire et particulièrement ceux portant sur l'établissement de conditions suffisantes à l'isolation des défauts mettent en relief les difficultés rencontrées en terme d'analyse et de formalisme.

Sur l'exemple des systèmes linéairement modélisés, un formalisme algébrique menant à la définition intrinsèque de l'isolabilité des défauts est présenté. Cependant ceci ne constitue que les prémisses de recherche qui pourraient mener à des définitions comparables pour les modèles non linéaires.

En tant que perspective à court terme, il serait également intéressant d'intégrer l'algorithme (o.c.a.) proposé par C. De Persis au sein de nos propositions afin d'augmenter le sous-espace d'état considéré lors de la génération des résidus.

Naturellement, supposer le système parfaitement modéliser constitue l'une des hypothèses dont nous souhaiterions nous affranchir dans l'avenir. Cependant, l'étude de modèles incertains est généralement synonyme de minimisation de critère ou encore d'étude de sensibilité. Ce problème est particulièrement complexe à résoudre lorsque les modèles sont non linéaires. Ensuite, nous voudrions intégrer la procédure de diagnostic à la commande des systèmes en boucle fermée. Ceci nécessite la synthèse conjointe des modules de diagnostic et de commande et donc l'étude des interactions commande-diagnostic. Nous ajoutons que de prime abord, dans le cadre de systèmes parfaitement modélisés ceci semble trivial. En revanche, les difficultés apparaissent dans le cadre de l'étude des modèles incertains. 


\section{Annexe A}

\section{Algèbre}

\section{Matrice jacobienne}

Notons $d h$ la matrice jacobienne de la fonction vectorielle de $h(x(t))$ égale à :

$$
d h=\frac{\partial h(x(t))}{\partial x(t)}=\left[\begin{array}{ccccc}
\frac{\partial h_{1}(x(t))}{\partial x_{1}(t)} & \cdots & \frac{\partial h_{1}(x(t))}{\partial x_{i}(t)} & \cdots & \frac{\partial h_{1}(x(t))}{\partial x_{n}(t)} \\
\vdots & \ddots & \vdots & & \vdots \\
\frac{\partial h_{i}(x(t))}{\partial x_{1}(t)} & \cdots & \frac{\partial h_{i}(x(t))}{\partial x_{i}(t)} & \cdots & \frac{\partial h_{i}(x(t))}{\partial x_{n}(t)} \\
\vdots & & \vdots & \ddots & \vdots \\
\frac{\partial h_{p}(x(t))}{\partial x_{1}(t)} & \cdots & \frac{\partial h_{p}(x(t))}{\partial x_{i}(t)} & \cdots & \frac{\partial h_{p}(x(t))}{\partial x_{n}(t)}
\end{array}\right]
$$

Dérivée de Lie

La dérivée de Lie du vecteur $\tau(x(t))$ le long du champ $f(x(t))$ est :

$$
L_{f} \tau(x(t))=\frac{\partial \tau(x(t))}{\partial x(t)} f(x(t))
$$

avec les opérations suivantes :

$$
\begin{aligned}
& L_{g} L_{f} \tau(x(t))=\frac{\partial L_{f} \tau(x(t))}{\partial x(t)} g(x(t)) \\
& L_{f}^{k} \tau(x(t))=\frac{\partial L_{f}^{k-1} \tau(x(t))}{\partial x(t)} f(x(t))
\end{aligned}
$$

$\underline{\text { Dérivée du co-vecteur } \omega(x(t)) \text { le long du champ } f(x(t)) \text { : }}$

$$
L_{f} \omega(x(t))=\left(\frac{\partial \omega^{T}(x(t))}{\partial x(t)} f(x(t))\right)^{T}+\omega(x(t)) \frac{\partial f(x(t))}{\partial x(t)}
$$

$\underline{\text { Crochet de Lie }}$

$$
[f(x(t)), g(x(t))]=L_{f} g(x(t))-L_{g} f(x(t))=\frac{\partial g(x(t))}{\partial x(t)} f(x(t))-\frac{\partial f(x(t))}{\partial x(t)} g(x(t))
$$


Définition A.1 Une distribution $\Delta=\operatorname{span}\left\{\tau_{1}, \cdots, \tau_{n}\right\}$ sur une variété $M$ de dimension $m$ est involutive si : $\forall i, j \in n$

$$
\left[\tau_{i}, \tau_{j}\right]=\sum_{k \in n} f_{i j}^{k}(x) \tau_{k}
$$

où $f_{i j}^{k}(x)$ représentent des fonctions $C^{\infty}$ définies sur le voisinage considéré.

Théorème A.1 (Théorème de Frobenius) Une distribution $\Delta$ sur une variété $M$ de dimension $m$ est complètement integrable $\Longleftrightarrow$ elle est involutive.

Définition A.2 Un changement de coordonnés $(z(t)=\Phi(x(t)))$ est appelé difféomorphisme global sur $\mathbb{R}^{n}$ où $\Phi(x(t))$ s'écrit de la manière suivante :

$$
\Phi(x(t))=\left(\begin{array}{c}
\Phi_{1}(x(t)) \\
\Phi_{2}(x(t)) \\
\vdots \\
\Phi_{n}(x(t))
\end{array}\right)=\left(\begin{array}{c}
\Phi_{1}\left(x_{1}(t), \cdots, x_{n}(t)\right) \\
\Phi_{2}\left(x_{1}(t), \cdots, x_{n}(t)\right) \\
\vdots \\
\Phi_{n}\left(x_{1}(t), \cdots, x_{n}(t)\right)
\end{array}\right)
$$

et possède les propriétés suivantes :

(i) $\Phi(x(t))$ est inversible, c'est-à-dire qu'il existe une fonction $\Phi^{-1}(z(t))$ telle que $\Phi^{-1}(\Phi(x(t)))=x(t)$.

(ii) $\Phi(x(t))$ et $\Phi^{-1}(z(t))$ sont deux métriques lisses (smooth mappings en Anglais), c'est-àdire toutes leurs dérivées partielles sont continues. 


\section{Annexe B}

\section{Géométrie}

Pour faciliter la lecture nous rappelons ici les modèles des systèmes utilisés dans cette annexe.

- les systèmes linéaires (B.1),

$$
\Sigma_{L}:\left\{\begin{array}{l}
\dot{x}(t)=A x(t)+B u(t) \\
y(t)=C x(t)
\end{array}\right.
$$

- les systèmes non linéaires affines en la commande (B.2),

$$
\Sigma_{N L}:\left\{\begin{array}{l}
\dot{x}(t)=f_{0}(x(t))+\sum_{i=1}^{m} f_{i}(x(t)) u_{i}(t) \\
y(t)=h(x(t))
\end{array}\right.
$$

$\Delta$ est dit invariant pour la dynamique du système (B.1) si, et seulement si, $A \Delta \subseteq \Delta$. Nous parlerons de sous-espace vectoriel $A$-invariant.

Nous étendons cette notion aux modèles non linéaires. $\Delta$ est dit invariant pour la dynamique du système (B.2) si, et seulement si, $\left[f_{i}, \Delta\right] \subseteq \Delta \forall i \in\{0, \cdots, m\}$. Nous parlerons de sousespace vectoriel $\left(f_{i}\right)$-invariant.

Définition B.1 (Définition de l'espace observable) Considérons le système non linéaire (B.2). L'espace observable $\gamma_{o b s}$ est l'espace linéaire (sur $\left.\mathbb{R}\right)$ des fonctions contenant $h_{1}, \cdots, h_{p}$ et toutes les répétitions des dérivées de Lie

$$
L_{X_{1}} L_{X_{2}} \cdots L_{X_{k}} h_{j}, \quad j \in\{1, \cdots, p\}, k=\{1,2, \cdots\}
$$

avec $X_{i}, i \in k$, dans l'ensemble $\left\{f_{0}, \cdots, f_{m}\right\}$

Cette définition est donnée dans [56] page 94. D'autres définitions de l'espace d'observabilité peuvent être déclinées à partir de cette dernière.

Nous ajoutons que l'espace d'observbilité est un sous-espace d'état généré par les distributions notées $d \gamma_{o b s}$. 
Théorème B.1 (Observabilité théorème du rang) Considérons le système (B.2) avec $\operatorname{dim}(x(t)=n)$. Supposons que

$$
\operatorname{dim}\left(d \gamma_{o b s}\left(x_{0}(t)\right)\right)=n
$$

alors le système est localement observable en $x_{0}(t)$.

Dans ce mémoire, nous utilisons régulièrement les notions de sous-espace d'état et de base originelle. Ainsi, pour l'écriture d'un système tel que celui défini équation (B.2), la base originelle de l'espace d'état est :

$$
\text { Base }_{\text {originelle }}=\left(\frac{\partial}{\partial x_{1}(t)}, \frac{\partial}{\partial x_{2}(t)}, \cdots, \frac{\partial}{\partial x_{n}(t)}\right)
$$

Ainsi, par exemple, la fonction $x_{1}(t)$ est représentée par le vecteur $\left(\begin{array}{llll}1 & 0 & 0 & 0\end{array}\right)$ dans cet espace.

Suite à la définition de la base d'origine il convient de définir les changements de base (ou de coordonnées) que nous appelons difféomorphisme pour les raisons expliquées en Annexe A. Ainsi, soit $\tilde{x}(t)=\Phi(x(t))$, le nouveau système de coordonnées est alors :

$$
\text { Base }_{\text {transformée }}=\left(\frac{\partial}{\partial \tilde{x}_{1}(t)}, \frac{\partial}{\partial \tilde{x}_{2}(t)}, \cdots, \frac{\partial}{\partial \tilde{x}_{n}(t)}\right)
$$

Exemple :

Soit $\Phi(x(t))=\left(x_{1}(t)+x_{2}(t) \quad x_{1}(t)-x_{2}(t)\right)^{T}$, en considérant la fonction $x_{1}(t)$, nous obtenons après transformation $1 / 2\left(\tilde{x}_{1}(t)+\tilde{x}_{2}(t)\right)$ ce qui a pour représentation dans la base transformée : $\left(\begin{array}{llll}0.5 & 0.5 & 0 & 0\end{array}\right)$. 


\section{Annexe C}

\section{Algèbre commutative}

\section{C.1 Relation d'équivalence}

Soit $\mathcal{R}$ une relation et $E$ un ensemble.

Relation d'équivalence :

$\widehat{\mathcal{R}}$ est une relation d'équivalence si, et seulement si, les propriétés suivantes sont satisfaites :

(i) $\mathcal{R}$ est transitive : pour tous $x, y, z$ de $E$ si $x \mathcal{R} y$ et $y \mathcal{R} z$ alors $x \mathcal{R} z$

(ii) $\mathcal{R}$ est réflexive : pour tous $x$ de $E$ si $x \mathcal{R} x$

(iii) $\mathcal{R}$ est symétrique : pour tous $x, y$ de $E$ si $x \mathcal{R} y$ alors $y \mathcal{R} x$

Classe d'équivalence :

La classe d'équivalence de $x$ relativement à une relation d'équivalence $\mathcal{R}$ est l'ensemble des éléments en relation avec $x: C(x)=\{y \in E, x \mathcal{R} y\}$.

\section{Partition :}

L'ensemble des classes d'équivalence d'un ensemble $X$ pour une relation d'équivalence $\mathcal{R}$ définie une partition de $X$.

Quotient d'un ensemble :

Nous appelons ensemble quotient de l'ensemble $X$ pour la relation d'équivalence $\mathcal{R}$ l'ensemble des classes d'équivalence de la relation $\mathcal{R}$. Nous notons cet ensemble $X / \mathcal{R}$. A tout élément de $X$ nous pouvons associer la classe d'équivalence correspondante.

\section{C.2 Groupe}

Groupe :

Un groupe $G$ est un ensemble muni d'une loi de composition interne associative (notée $\star$ ), 
ayant un élément neutre 0 et telle que tout élément ait un symétrique á.

si $(a, b, c) \in G$ alors :

(i) $(a \star b) \star c=a \star(b \star c)$

(ii) $a \star b=b \star a$

(iii) $0 \star a=a \star 0=a$

(iv) $a \star(\dot{a})=(\dot{a}) \star a=0$

avec (ii) nous définissons un groupe abélien ou commutatif.

\section{C.3 Anneau}

Anneau :

Un anneau $A$ est un ensemble muni de deux lois de compositions internes notées $\star$ (addition) et $\diamond$ (multiplication) telles que :

(i) $(A, \star)$ est un groupe abélien

(ii) $\diamond$ est associative, i.e. $(x, y, z) \in A(x \diamond y) \diamond z=x \diamond(y \diamond z)$

(iii) $\diamond$ est distributive par rapport à $\star$, i.e. $x \diamond(y \star z)=x \diamond y \star x \diamond z$ et $(y \star z) \diamond x=y \diamond x \star z \diamond x$

Diviseur de zéro :

Soit $(A, \star, \diamond)$ un anneau. Un élément $x$ de $A$ est un diviseur de zéro si :

$$
\exists y \in A, y \neq 0 \quad x \diamond y=0
$$

\section{Anneau commutatif :}

L'anneau est dit commutatif si la loi $\diamond$ est commutative.

\section{Anneau unitaire :}

L'anneau est dit unitaire si la loi $\diamond$ admet un élément neutre.

\section{Anneau nul :}

Si l'anneau est réduit à l'élément neutre de la loi $\star$, on dit que l'anneau est nul.

Anneau intègre :

On appelle anneau intègre un anneau non nul sans diviseur de zéro autre que 0 .

\section{Idéal :}

On appelle idéal une partie $I$ de $A$ qui est un sous groupe additif de $A$, stable pour le produit 
par un élément de $A$.

L'idéal $I$ est dit principal si il existe un élément $\alpha$ tel que $I$ soit l'ensemble des multiple de $\alpha$, i.e.

$$
I=\{x \in A \mid \exists a \in A \text { et } x=a \diamond \alpha\}
$$

dans ce cas on dira que l'idéal $I$ est engendré par $\alpha$.

\section{C.4 Corps}

Corps :

Un corps $K$ un ensemble muni de deux lois de compositions internes notées $\star$ et $\diamond$ telles que :

(i) $(K, \star, \diamond)$ est un anneau

(ii) l'élément neutre de $\star$ est différent de l'élément neutre de $\diamond$

(iii) tout élément de $(K-\{0\}, \diamond)$ est inversible

\section{C.5 Module}

\section{Module :}

Soit un anneau commutatif $A(\star, \diamond)$, un $A$-module $M$ est un ensemble muni de deux opérations, la première $M \times M \longrightarrow M$ notée + est appelée l'addition. La seconde $A \times M \longrightarrow M$ notée . est appelée la multiplication externe (à droite), telle que avec $a, b \in A$ et $x, y, z \in M$ :

(1) $M$ muni de l'addition $(x, y) \longrightarrow x+y$ est un groupe abélien, i.e. :

(i) $x+(y+z)=(x+y)+z$

(ii) $x+y=y+x$

(iii) $x+0=0+x=x$ où l'élément 0 est l'élément neutre pour l'addition

(iv) $x+(-x)=(-x)+x=0$

(2) $M$ muni de la multiplication externe $(a, x) \longrightarrow a . x$

(i) $(a \diamond b) \cdot x=a \cdot(b \cdot x)$ (associativité mixte)

(ii) $1 . x=x$ où l'élément 1 est l'élément neutre pour la multiplication

(3) La multiplication externe . est doublement distributive sur l'addition, i.e. :

(i) $(a \star b) \cdot x=a \cdot x+b \cdot x$

(ii) $a \cdot(x+y)=a \cdot x+a \cdot y$

En remarque, nous ajoutons que si $A$ est un corps commutatif alors le $A$-module est un $A$-espace vectoriel. 
Soit $M$ un $A$-module, un sous-groupe $N$ de $M$ tel que $a x \in N$ pour $a \in A$ et $x \in N$ est un sous-module de $M$.

L'ensemble des éléments $\left(e_{i}\right)$ forme une base de $M$ si, seulement si, tout élément de $M$ s'écrit de façon unique comme combinaison linéaire des $e_{i}$.

Module libre :

Un $A$-module admettant une base est libre.

Elément de torsion :

Etant donné un anneau commutatif et unitaire $A$ et un $A$-module $M$, un élément $x$ de $M$ est dit de torsion s'il existe un élément $a$ non nul de $A$ tel que $a . x=0$.

\section{Sous-module de torsion :}

Etant donné un anneau commutatif et unitaire $A$ et un $A$-module $M$, on appelle sous-module de torsion de $M$ l'ensemble des éléments de torsion de $M$.

\section{Module sans torsion:}

Etant donné un anneau commutatif unitaire et intègre $A$, un $A$-module $M$ est dit sans torsion si son sous-module de torsion est nul.

\section{Module fini :}

Si $M$ est engendré par un nombre fini d'éléments, nous disons que $M$ est un module de type fini.

\section{C.6 Morphisme}

Morphisme de groupe :

Soient $(G,+)$ et $(H, t)$ deux groupes, une application $f$ de $G$ dans $H$ est un morphisme (nous rencontrons aussi homomorphisme) de groupe si, et seulement si, pour tout $x \in G, y \in G$ nous avons $f(x+y)=f(x) t f(y)$.

Morphisme d'anneau :

Soient $(A,+,$.$) et (B, \star, \diamond)$ deux anneaux, une application $f$ de $A$ dans $B$ est un morphisme (nous rencontrons aussi homomorphisme) d'anneau si, et seulement si, les propriétés suivantes sont vérifiées :

(i) $\mathrm{f}(1)=1$ où 1 est l'élément neutre de l'opération

(ii) pour tout $x \in A, y \in B$ nous avons $f(x+y)=f(x) \star f(y)$ 
(iii) pour tout $x \in A, y \in B$ nous avons $f(x . y)=f(x) \diamond f(y)$

Morphisme de $A$-module :

Soient $M$ et $N$ deux $A$-modules, une application $f$ de $M$ dans $N$ est un morphisme de $A$ modules si, et seulement si, la propriété suivante est vérifiée $: f(x . a+y \cdot b)=f(x) \cdot a+f(y) \cdot b$.

Isomorphisme :

Un morphisme $f$ de $S$ dans $S^{\prime}$ est appelé isomorphisme s'il admet un morphisme inverse, c'est-à-dire s'il existe $g$ de $S^{\prime}$ dans $S$ telle que $g \circ f=I_{S}$ et $f \circ g=I_{S^{\prime}}$.

Endomorphisme :

Un morphisme $f$ de $S$ dans $S$ est un endomorphisme.

Automorphisme :

Si l'endomorphisme $f$ est un isomorphisme alors $f$ est un automorphisme.

\section{C.7 Diagramme commutatif}

\section{Suites exactes :}

Nous appellons suite exacte de $A$-modules une collection d'applications A-linéaires du type :

$$
\cdots \longrightarrow M_{-2} \stackrel{u_{-2}}{\longrightarrow} M_{-1} \stackrel{u_{-1}}{\longrightarrow} M_{0} \stackrel{u_{0}}{\longrightarrow} M_{1} \stackrel{u_{1}}{\longrightarrow} M_{2} \stackrel{u_{2}}{\longrightarrow} \cdots
$$

telle que $\operatorname{ker}\left\{u_{n+1}\right\}=\operatorname{im}\left\{u_{n}\right\}$ pour tout $n \in \mathbb{Z}$.

Notons par abus 0 le $A$-module.

$\underline{\text { Suite exacte courte : }}$

Une suite exacte courte est une suite exacte du type :

$$
0 \longrightarrow M^{\prime} \stackrel{f}{\longrightarrow} M \stackrel{g}{\longrightarrow} M^{\prime \prime} \longrightarrow 0
$$

on dit que $M$ est une extension de $M^{\prime \prime}$ par $M^{\prime}$.

En remarque nous ajoutons que :

- considérer $0 \longrightarrow M^{\prime} \stackrel{f}{\longrightarrow} M$ exacte signifie que $f$ est injective.

- considérer $M \stackrel{g}{\longrightarrow} M^{\prime \prime} \longrightarrow 0$ exacte signifie que $g$ est surjective.

\section{C.8 Foncteur}

Catégories :

Une catégorie $\mathcal{V}$ consiste en une classe d'objets, une classe de morphismes et une loi de 
composition de morphismes.

\section{Foncteur :}

Soient deux catégories $\mathcal{V}$ et $\mathcal{W}$. Un foncteur covariant $T$ de $\mathcal{V}$ vers $\mathcal{W}$, noté $T: \mathcal{V} \rightarrow \mathcal{W}$, consiste en deux applications ensemblistes (notées également $T$ ) :

(i) une application qui associe à tout objet $V \in \mathcal{V}$ un objet $T(V) \in \mathcal{W}$

(ii) une application qui associe à tout morphisme $f \in \mathcal{V}, f: \mathcal{V} \rightarrow \mathcal{W}$ un morphisme $T(f) \in \mathcal{W}$ de la forme $T(f): T(\mathcal{V}) \rightarrow T(\mathcal{W})$ 


\section{Bibliographie}

[1] M. Adam-Medina, D. Theilliol, et D. Sauter. Diagnostic de défauts des systèmes non linéaires décrits par des modèles linéaires interpolés. Dans Conférence Internationale Francophone d'Automatique CIFA 2002, pages 317-322, Nante - France, Juillet 2002.

[2] E. Alcorta-Garcia et P. M. Frank. Deterministic nonlinear observer-based approaches to fault diagnosis : a survey. Control Engineering Practice, 5(5) : 663-670, 1997.

[3] E. Alcorta-Garcia et P. M. Frank. Fault detection and isolation in nonlinear systems. Dans $5^{e}$ European Control Conference, numéro F246, Karlsruhe, Allemagne, Septembre 1999.

[4] D. AuBRY. Contribution à la synthèse d'observateurs pour les systèmes non linéaires. Thèse de doctorat, Université Henri Poincaré, Nancy, France, Juin 1999.

[5] M. Basseville. On-board component fault detection and isolation using the statistical local approach. Automatica, 34(11) : 1391-1415, 1998.

[6] M. Basseville et V. Nikiforov. Detection of abrupt changes : theory and application. Prentice Hall information and system sciences series. Prentice Hall, Englewood Cliffs, 1993.

[7] R. V. BEARD. Failure accomodation in linear systems through self-reorganization. Thèse de doctorat, Massachusette Institute of Technologiy. Departement of Aeronautics and Astronautics, Cambridge, Massachusetts (USA), Fevrier 1971.

[8] G. Besançon. A nonlinear observer with disturbance attenuation and its application to fault detection. Dans $5^{e}$ European Control Conference, numéro F1054-2, Karlsruhe, Allemagne, Septembre 1999.

[9] M. Boutayeb, H. Rafaralahy, et M. Darouach. Convergence analysis of extended kalman filter used as an observer for nonlinear deterministic discrete-time system. IEEE Transaction on Automatic Control, 42(4) : 581-586, 1997.

[10] I. N. Bronshteim et K. A. Semendyayev. Handbook of mathematics. Springer-Verlag, Berlin, Heidelberg, New York, $3^{\text {e }}$ édition, 1997. 
[11] J. P. Cassar, R. G. Litwak, V. Cocquempot, et M. Staroswiecki. Approche structurelle de la conception de systèmes de surveillance pour les procédés industriels complexes. Diagnostic et Sûreté de Fonctionnement, 4(2) : 179-202, 1994.

[12] J. Chen et R. J. Patton. Robust model-based fault diagnosis for dynamic systems, volume 3 de Kluwer international series on Asian studies in computer and information science. Kluwer Academic Publishers, Boston, Dordrecht, London, 1999.

[13] C. De Persis et A. Isidori. On the problem of residual generation for fault detection in nonlinear systems and some related facts. Dans $5^{e}$ European Control Conference, numéro F0827, Karlsruhe, Allemagne, Septembre 1999.

[14] C. De Persis et A. Isidori. A geometric approach to nonlinear fault detection and isolation. Dans $4^{e}$ IFAC Symposium on Fault Detection Supervision and Safety for Technical Processes SAFEPROCESS, volume 1, pages 209-214, Budapest, Hongrie, Juin 2000.

[15] C. De Persis et A. IsIDORI. On the observability codistributions of a nonlinear system. Systems \&f Control Letters, 40 : 297-304, 2000.

[16] C. De Persis et A. IsIdori. A geometric approach to nonlinear fault detection and isolation. IEEE Transaction on Automatic Control, 46(6) : 853-865, 2001.

[17] C. DE PERsis et A. Isidori. On the design of fault detection filters with gametheoretic-optimal sensitivity. International Journal of Robust and Nonlinear Control, $12: 729-747,2002$.

[18] F. Fairman, S. Mahil, et L. Luk. Disturbance decoupled observer design via singular value decomposition. IEEE Transactions on Automatic Control, 24(1) : 84-89, 1984.

[19] M. FLIESS. Some basic structural properties of generalized linear systems. Systems $\&$ Control Letters, 15 : 391-396, 1990.

[20] M. FLIESS. Generalized controller canonical forms for linear and nonlinear dynamics. IEEE Transaction on Automatic Control, 46(6) : 853-865, 2001.

[21] M. Fliess, J. Lévine, P. Martin, et P. Rouchon. Flatness and defect of nonlinear systems : introductory theory and applications. International Journal of Control, $61: 1327-1361,1995$.

[22] M. Fliess, J. Lévine, P. Martin, et P. Rouchon. A lie-bäcklund approach to equivalence and flatness of nonlinear systems. IEEE Transaction on Automatic Control, 44(5) : 922-937, 1999.

[23] M. Fliess, R. Marquez, E. Delaleau, et H. Sira-Ramirez. Correcteurs proportionnels-intégraux généralisés. ESAIM : Control, Optimisation an Calculus of Variations, 200X, will be set by publisher.

[24] M. Fliess et H. Mounier. Controllability and observability of linear delay systems : an algebraic approach. ESAIM : Control, Optimisation an Calculus of Variations, 3 : $301-$ $314,1998$. 
[25] A. J. Fossard et D. Normand-Cyrot. Nonlinear systems, volume 1-3. Chapman \& Hall, London, New York, 1995.

[26] P. M. Frank. Fault diagnosis in dynamic systems using analytical and knowledge-based redundancy a survey and some new results. Automatica, 26(3) : 459-474, 1990.

[27] P. M. FRANK et X. Ding. Survey of robust residual generation and evaluation methods in observer-based fault detection systems. Journal of Process Control, 7(6) : 403-424, 1997.

[28] B. FRIEDland. A nonlinear observer for estimating parameters in dynamic systems. Automatica, 33(8) : 1525-1530, 1997.

[29] B. Gaddouna. Contribution au diagnostic des systèmes linéaires invariants à entrées inconnues. Application à un processus hydraulique. Thèse de doctorat, Institut National Polytechnique de Lorraine, Nancy, France, Avril 1995.

[30] B. Gaddouna, D. Maquin, et J. Ragot. Fault detection observer for systems with unknown inputs. Dans $4^{e}$ IFAC Symposium on Fault Detection Supervision and Safety for Technical Processes SAFEPROCESS, volume 1, pages 69-74, Espoo, Finland, Juin 1994.

[31] J. J. GerTLER. Fault detection and diagnosis in engineering systems. Marcel Dekker, New York, 1998.

[32] L. Gross. Approches multi-modèles de la génération de résidus pour le diagnostic de systèmes. Thèse de doctorat, Université Henri Poincaré, Nancy, France, Janvier 1999.

[33] H. Hammouri, M. Kinnaert, et E. H. El YaAgoubi. Fault detection and isolation for state affine systems. European Journal of Control, 4(1) : 2-16, 1998.

[34] H. Hammouri, M. Kinnaert, et E. H. El YaAgoubi. Observer based approach to fault detection and isolation for non linear systems. IEEE Transaction of Automatic Control, 44(10) : 1879-1884, 1999.

[35] H. Hammouri, M. Kinnaert, et E. H. El YaAgoubi. A geometric approach to fault detection and isolation for bilinear systems. IEEE Transaction on Automatic Control, 46(9) : 1451-1455, 2001.

[36] R. Isermann et P. Ballé. Trends in the application of model-based detection and diagnosis of technical process. Control Engineering Practice, 5(5) : 709-719, 1997.

[37] A. IsIDORI. Nonlinear control systems. Communications and control engineering series. Springer-Verlag, Berlin, New York, $3^{\mathrm{e}}$ édition, 1995.

[38] A. Isidori, A. J. Krener, C. Gori-Giorgi, et S. Monaco. A geometric approach to synthesis of failure detection filters. IEEE Transaction on Automatic Control, 26(2) : 331-345, 1981. 
[39] C. Join, J. Jouffroy, J.-C. Ponsart, et J. Lottin. Synthèse d'un filtre isolateur de défauts à l'aide de la théorie de la contraction. Dans Conférence Internationale Francophone d'Automatique, pages 153-158, Nantes, France, Juillet 2002.

[40] C. Join, J.-C. Ponsart, et D. Sauter. Fault detection and isolation via nonlinear filters. Dans $15 t^{e}$ IFAC World Congress on Automatic Control, numéro 3000, Barcelone, Espagne, Juillet 2002.

[41] C. Join, J.-C. Ponsart, et D. Sauter. Sufficient conditions to fault isolation in nonlinear systems : a geometric approach. Dans $15^{e}$ IFAC World Congress on Automatic Control, numéro 2978, Barcelone, Espagne, Juillet 2002.

[42] C. Join, J.-C. Ponsart, D. Theilliol, et D. Sauter. Actuator and sensor fault diagnosis by decoupling method for nonlinear systems. Dans $2^{e}$ Nonlinear Control Network, Paris, France, Juillet 2000.

[43] H. L. Jones. Failure detection in linear systems. Thèse de doctorat, Massachusette Institute of Technologiy. Departement of Aeronautics and Astronautics, Cambridge, Massachusetts (USA), Août 1973.

[44] J. Jouffroy et J. LotTin. On the use of contraction theory for the design of nonlinear observers for ocean vehicles. Dans American Control Conference, volume 4, pages 2647 2652, Anchorage, Alaska, Mai 2002.

[45] P. KaBORE. Contribution à la detection et l'isolation de pannes dans les systèmes non linéaires. Thèse de doctorat, Université Claude Bernard, Lyon, France, Juillet 1997.

[46] T. Kailath. Linear systems. Prentice Hall information and system science series. Prentice Hall, Englewood Cliffs, 1980.

[47] V. Krishnaswami et G. Rizzoni. Nonlinear parity equation based residual generation for fault detection and isolation. Dans $4^{e}$ IFAC Symposium on Fault Detection Supervision and Safety for Technical Processes SAFEPROCESS, pages 317-322, Espoo, Finland, Juin 1994.

[48] B. LiU et J. Si. Fault isolation filter design for linear time-invariant systems. IEEE Transaction on Automatic Control, 42(5) : 704-707, 1997.

[49] W. Lohmiller et J.J. E. Slotine. Fault diagnosis in dynamic systems using analytical and knowledge-based redundancy a survey and some new results. Automatica, 34(6) : 683-696, 1998.

[50] J-F. Magni et P. Mouyon. On residual generation by observer and parity space approches. IEEE Transaction on Automatic Control, 39(2) : 441-447, 1994.

[51] M.-A. Massoumnia. A geometric approach to synthesis of failure detection filters. IEEE Transaction on Automatic Control, 31(9) : 839-846, 1986.

[52] M.-A Massoumnia, G. C. Verghese, et A. S. Willsky. Failure detection and identification. IEEE Transaction on Automatic Control, 34(3) : 316-321, 1989. 
[53] E. A. Misawa et J. K. HEDRICK. Nonlinear observer-a-state-of-the-art survey. Transaction of the American Society of Mechanical Engineering, 111 : 687-699, 1989.

[54] K. S. NARENDRA. Adaptive control of discrete-time systems using multiple models. IEEE Transactions on Automatic Control, 45(9) : 1669-1685, 2000.

[55] K. S. Narendra, J. Balakrishnan, et M. K. Ciliz. Adaptation and learning using multiple models, switching, and tuning. IEEE Control Systems, 15(3) : 37-51, 1995.

[56] H. Nijmeijer et A. J. VAN DeR SChaft. Nonlinear dynamical control systems. Springer-Verlag, New York, 1996.

[57] J. Park, G. Rizzoni, et W. B. Ribbens. On the representation of sensor faults in fault detection filters. Automatica, 30(11) : 1793-1795, 1994.

[58] R. Patton, P. M. Frank, et R. Clark. Fault diagnosis in dynamic systems. Theory and application. Prentice Hall International series in systems and control engineering. Prentice Hall, Englewood Cliffs, 1989.

[59] R. Patton, P. M. Frank, et R. Clark. Issues of fault diagnosis for dynamic systems. Springer-Verlag, London, New York, 2000.

[60] J-C. Ponsart, C. Join, D. Theilliol, et D. Sauter. Sensor fault diagnosis and accommodation in nonlinear system. Dans $6^{e}$ European Control Conference, pages $2346-$ 2351, Porto, Portugal, Septembre 2001.

[61] K. Reif et R. Unbehauen. The extended kalman filter as an exponential observer for nonlinear systems. IEEE Transaction on Signal Processing, 47(8) : 2324-2328, 1999.

[62] A. Y. SHumsky. Nonlinear observer-based parameter estimation and fault diagnosis in dynamic systems. Dans $5^{e}$ European Control Conference, numéro F1053-4, Karlsruhe, Allemagne, Septembre 1999.

[63] E. D. SontaG. Mathematical control theory, volume 6 of Texts in Applied Mathematics. Springer-Verlag, New York, Berlin, Heidelberg, $2^{\text {e }}$ édition, 1998.

[64] A. S. Willsky. A survey of design methods for failure detection in dynamic systems. Automatica, 12(6) : 601-611, 1976.

[65] W. M. Wonham. Linear multivariable control : a geometric approach. volume 10 of Applications of Mathematics. Springer-Verlag, New York, Berlin, Heidelberg, $2^{\mathrm{e}}$ édition, 1985.

[66] A. ZolghadRI. A redundancy-based strategy for safety management in a modern civil aircraft. Control Engineering Practice, 8(5) : 545-554, 2000.

[67] A. Zolghadri, D. Henry, et M. Monsion. Design of nonlinear observers for fault diagnosis : a case study. Control Engineering Practice, 4(11) : 1535-1544, 1996. 




\section{Résumé}

Le travail présenté dans ce mémoire porte sur le diagnostic de systèmes non linéaires en temps continu. Dans le but de déterminer le type et l'apparition de défauts, des résidus structurés sont générés à partir de filtres, en respectant les étapes suivantes :

- la première étape est une phase de découplage d'une partie de l'état du système de l'effet des perturbations. Nous proposons une méthode qui permet de diminuer la dimension de ce sous-espace d'état de manière à limiter au maximum la propagation de ces perturbations dans l'espace d'état; - la deuxième étape est l'étude de l'ensemble des sous-espaces d'état sensibles aux défauts considérés dans le but de déterminer s'il est possible de les détecter et isoler. En supposant la non-apparition simultanée de plusieurs défauts, une analyse structurelle des caractéristiques géométriques du système est menée. Ainsi, des conditions nécessaires et suffisantes à l'isolation des défauts moins sévères que celles associées à la résolution du F.P.R.G. sont proposées;

- la troisième et dernière étape est la synthèse d'un filtre d'isolation des défauts considérés. Une méthode systématique assurant une expression explicite de l'injection de sortie est proposée. Une étude de la convergence des estimations de l'état du filtre vers l'état réel du système est également effectuée à partir de la théorie de la contraction.

L'ensemble de la méthode est appliquée sur le système des " 3 cuves". Des simulations mettent en relief les résultats obtenus et l'apport des méthodes proposées.

Le dernier chapitre porte sur les prémices d'un formalisme algébrique du diagnostic pour les systèmes linéaires qui semble être une perspective intéressante pour les systèmes non linéaires.

Mots clés : systèmes non linéaires, diagnostic, filtre, découplage, analyse de convergence, approche géométrique.

\section{Summary}

The main work presented in this document deals with the diagnosis of nonlinear systems in continuous time. The residual generation for fault isolation are considered with respect to the following steps:

- the first step consists of decoupling the disturbance effect from a part of the system state. We propose a method which aims at reducing the dimension of this state subspace in order to limit the propagation of disturbances in the subspace to a maximum;

- the second step is the study of the state subspaces sensitive to the considered faults with the aim of determining if it is possible to detect and isolate all faults. Assuming that faults do not occur simultaneously, isolation conditions less restrictive than those solving the F.P.R.G. are proposed; - the third step corresponds to the filter synthesis. A systematic method ensuring an explicit expression of the output injection is proposed. Moreover, a convergence study of the estimated state and the real state is included within the filter design.

These techniques are applied to the "three tank" system in simulation which highlight the obtained results.

Finally new results leading to one intrinsic definition of fault isolation in linear case have been presented and should be extended to nonlinear systems.

Keywords: nonlinear system, diagnosis, filter, decoupling method, convergence analysis, geometric approach. 